\title{
Liquid Crystals as High REPETITION RATE TARgETS FOR Ultra Intense LASER Systems
}

\author{
DISSERTATION
}

Presented in Partial Fulfillment of the Requirements for the Degree Doctor of Philosophy in the Graduate School of The Ohio State University

By

Patrick L. Poole, B.S. \& M.S.

Graduate Program in Physics

The Ohio State University

2015

Dissertation Committee:

Professor Douglass Schumacher, Advisor

Professor Lou DiMauro

Professor Ulrich Heinz

Professor Ezekiel Johnston-Halperin 
(C) Copyright by

Patrick L. Poole 


\section{ABSTRACT}

This thesis presents the development of and first experiments on freely suspended liquid crystal film targets for intense laser-matter experimentation. Liquid crystals exhibit additional phases between solid and liquid which are characterized by different levels of molecular ordering. One of these, the smectic phase, entails molecular orientational order and positional order such that the constituent molecules arrange into layers of set thickness. The surface tension inherent to this smectic phase allows a liquid crystal film to be formed within an aperture in a rigid frame; control over the parameters of film formation (temperature, volume, wiper speed, etc.) allows the number of layers comprising this freely suspended film to be modified on-demand. The result is a variable thickness target with planar geometry that is robust to target chamber vacuum environments and is also inexpensive due to the low volume used per film.

Initial ion acceleration experiments will be discussed, where the variable thickness of liquid crystal films is uniquely capable of characterizing the regions of dominance of various acceleration mechanisms. These experiments were performed on the Scarlet laser facility at the Ohio State University, which is a $400 \mathrm{TW}, 12 \mathrm{~J}, 30 \mathrm{fs}$ Ti:sapphire laser system that

produces focused intensities in excess of $10^{21} \mathrm{~W} / \mathrm{cm}^{2}$. The upgrade of this facility to these specifications including damage testing measurements on the new optics is also discussed.

Additionally, a device for the rapid insertion in-vacuo of these liquid crystal films into the target plane will be presented. This apparatus takes advantage of techniques developed for film formation in a single shot capacity but increases the repetition rate possible to the $0.1 \mathrm{~Hz}$ scale. Films formed with the device are done so within $2 \mu \mathrm{m}$ of the same position each time, which is critical for high repetition rate insertion where no time for target alignment is available. 
To friends and family near and far, without whom my life would be equal parts less successful and duller - and especially to Amanda 


\section{ACKNOWLEDGMENTS}

Those who have dedicated themselves to obtaining a $\mathrm{PhD}$, or another arduous commitment whose completion is years in the making, know that success is as much dependent on support from friends as on one's own efforts. Here is a list of appreciative thoughts (too brief to properly convey their assistance or my gratitude) for those who were most integral to helping me along the way through graduate school.

First, to those who came before me and guided the way: Dr. Link, Dr. Morrison, and Dr. Krieger. Whether you knew it or not I learned quite a bit from watching you and by striving to reach your levels.

To Enam, from whom I have learned much about lasers and the laboratory, and who pushed me to be a "demigod".

To Becky, who keeps the lab running smoothly and without whom many days would be darker and less entertaining.

To Chris, my partner in the laser mines-I couldn't have done half of it without you, and the other half I wouldn't have wanted to.

Collectively to the Scarlet laser team: Enam, Becky, Chris, Scott, Kevin, and others: the work presented in Chapter 4 represents all of our efforts over quite some time, and the result is a world-class laser facility. I'm proud of have worked on it with all of you.

To advisors past and present: Linn Van Woerkom, Kramer Akli, and Dave Andereck. Your guidance and friendship has meant a lot over the years, and I think if I ever learn half the things you've tried to teach me I'll be in good shape.

To Rick, who has taught me much and continues to do so. I could spend a decade working with you and still not learn most of what you know, or repay you for half the opportunities you've provided for me, but I have resolved to come as close as I can.

To Doug, who has been a better mentor and friend than he needed to be. I never had a stronger incentive to excel in my work than to earn the right to be this man's colleague.

To my many friends I've made as we all work towards our PhDs: if shared experience through mutual hardship makes fast friends, then I think I have made many that will last me a lifetime.

My acknowledgments would be incomplete without a deep thank you to my family. 
Though this work has kept me far away from you for many years now, I was able to continue through the tough times knowing that I had your love and support.

Lastly to Amanda-the brightest, wittiest, kindest person I know, to whom my thoughts always turn when I'm deep in work. 


\section{VITA}

October $22,1985 \ldots \ldots \ldots \ldots \ldots \ldots \ldots \ldots \ldots$ Born - Valdosta, GA

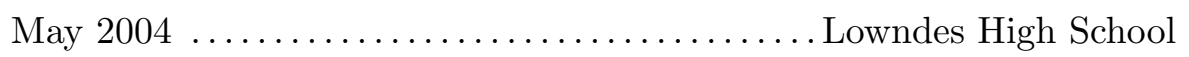

May 2008 .............................. B. in Physics with High Honors, Georgia Institute of Technology, Atlanta, Georgia

December 2008 ....................... B.S. in Applied Mathematics with Highest Honors, Georgia Institute of Technology, Atlanta, Georgia

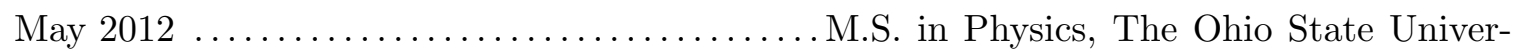
sity, Columbus, Ohio

\section{Publications}

"Liquid Crystal Thin-Film Laser Target" P. L. Poole, C. D. Andereck, D. W. Schumacher, Patent pending 62/082,911, (2014).

"Liquid crystal films as on-demand, variable thickness (50-5000 nm) targets for intense lasers" P. L. Poole, C. D. Andereck, D. W. Schumacher, R. L. Daskalova, S. Feister, K. M. George, C. Willis, K. U. Akli, E. A. Chowdhury, Physics of Plasmas 21, 063109 (2014).

"High repetition rate, liquid crystal thin-film target inserter" P. L. Poole, C. D. Andereck, D. W. Schumacher, Patent pending 62/001,698, (2014).

"Femtosecond laser damage threshold of pulse compression gratings for petawatt scale laser systems" P. L. Poole, S. Trendafilov, G. Shvets, D. Smith, and E. A. Chowdhury, Optics Express 21, 026341 (2013).

"Damage testing of critical optical components for high power ultra-fast lasers" E. A. Chowdhury, P. L. Poole, S. Jiang, B. Taylor, R. Daskalova, L. van Woerkom, R. R. Freeman, D. Smith, Proc. SPIE 7842, 78421Y, (2010). 


\section{Presentations}

"Liquid crystal film targets from $10 \mathrm{~nm}$ to $10 \mu \mathrm{m}$ thickness formed on-demand at the Scarlet laser facility" P. L. Poole, C. Willis, G. E. Cochran, M. McMahon, E. A. Chowdhury, C. D. Andereck, D. W. Schumacher, NNSA SSAP 2015, Santa Fe, NM (2015).

"Liquid crystal targets for high repetition rate applications" P. L. Poole, CETAL experimental workshop, Magurele, Romania (2015).

"Linear Slide Target Inserter for repeatable liquid crystal film target formation at $0.1 \mathrm{~Hz}$ " P. L. Poole, C. Willis, G. E. Cochran, R. J. Hanna, C. D. Andereck, D. W. Schumacher, NIF/JLF 2015 user group meeting, Livermore, CA (2015).

"Liquid crystals as variable thickness targets $(10 \mathrm{~nm}-10 \mu \mathrm{m})$ for repetition rated intense laser applications" P. L. Poole, C. Willis, G. E. Cochran, R. J. Hanna, C. D. Andereck, D. W. Schumacher, European XFEL workshop 2015, Hamburg, Germany (2015).

"Liquid crystals as variable thickness targets for high repetition rate intense laser applications" P. L. Poole, C. Willis, G. E. Cochran, R. J. Hanna, C. D. Andereck, D. W. Schumacher, DARPA PULSE meeting 2015, Arlington, VA (2015).

"Liquid crystals as on-demand, variable thickness targets for intense laser applications" P. L. Poole, C. D. Andereck, D. W. Schumacher, 2014 meeting of the APS Division of Plasma Physics, New Orleans, LA (2014).

"Liquid crystals as variable thickness targets for high repetition rate intense laser applications' P. L. Poole, C. Willis, G. E. Cochran, R. J. Hanna, C. D. Andereck, D. W. Schumacher, SLAC 2nd High Power Laser workshop, Palo Alto, CA (2014).

"First experimental data on liquid membrane targets for high repetition rate neutron generation" P. L. Poole, C. D. Andereck, D. W. Schumacher, OSU Physics Poster Competition, top prize, Columbus, $\mathrm{OH}$ (2014).

"Liquid crystal films for high repetition rate laser experiments at ELI" P. L. Poole, C. D. Andereck, D. W. Schumacher, ELI-ALPS experimental workshop, Szeged, Hungary (2014).

"First experimental data on liquid membrane targets for high repetition rate neutron generation" P. L. Poole, C. D. Andereck, E. A. Chowdhury, D. W. Schumacher, OMEGA user's group meeting, Rochester, NY (2014).

"Liquid membrane target for high repetition rate neutron generation" P. L. Poole, C. D. Andereck, D. W. Schumacher, DARPA PULSE winter review, Austin, TX (2014). 
"Liquid membrane target for high repetition rate neutron generation" P. L. Poole, C. D. Andereck, D. W. Schumacher, NIF/JLF 2014 user group meeting, Livermore, CA, (2014).

"Design of a liquid membrane target for high repetition rate neutron generation" P. L. Poole, M. Storm, E. A. Chowdhury, C. D. Andereck, D. W. Schumacher, 2013 meeting of the APS Division of Plasma Physics, Denver, CO, (2013).

"Scarlet petawatt class laser at the OSU High Energy Density Physics group" P. L. Poole, C. Willis, S. Feister, K .George, R. Daskalova, E. A. Chowdhury, R. R. Freeman, Frontiers in Extreme Relativistic Optics, Columbus, OH, (2013).

"Petawatt class ultra-high peak power laser design at OSU HEDP group" P. L. Poole, C. Willis, R. Daskalova, E. A. Chowdhury, R. R. Freeman, Fusion Science Center workshop, Livermore, CA (2011).

"Damage testing of critical optical components for high power ultra-fast lasers" P. L. Poole, R. Daskalova, E. A. Chowdhury, 4th biennial ICUIL conference, Watkins Glen, NY (2010).

\section{Fields of Study}

Major Field: Physics 


\section{Table of Contents}

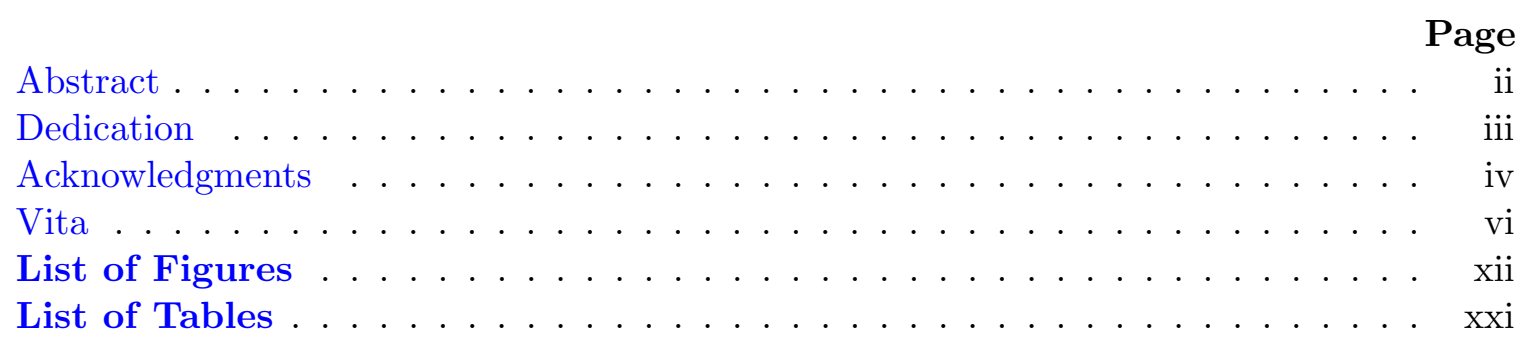

\section{Chapters}

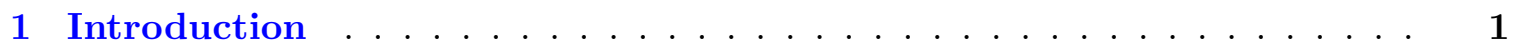

1.1 High Energy Density Physics . . . . . . . . . . . . . . . . 1

1.2 Applications . . . . . . . . . . . . . . . . . 2

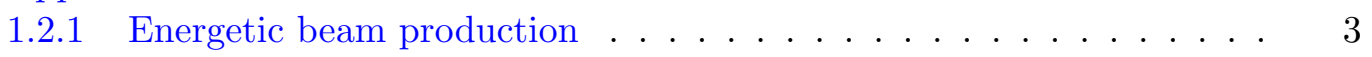

1.2.2 Hadron cancer therapy . . . . . . . . . . . . . . . . 4

1.3 High repetition rate lasers . . . . . . . . . . . . . . . . . 6

1.4 This Work . . . . . . . . . . . . . . . . . . 7

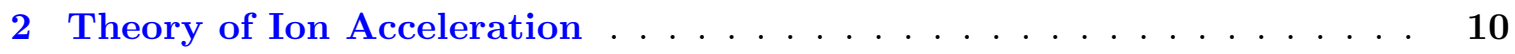

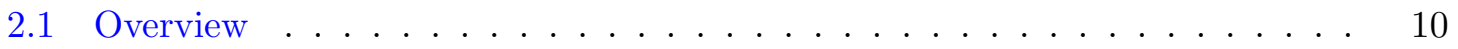

2.2 Initial laser interaction . . . . . . . . . . . . . . . . . . 11

2.2.1 Laser pulse shape . . . . . . . . . . . . . . . . . . 11

2.2 .2 Laser-plasma interactions . . . . . . . . . . . . . . . . . . . . . 12

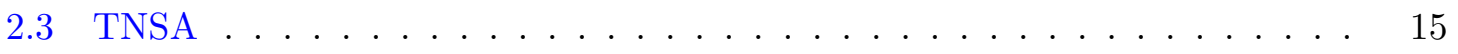

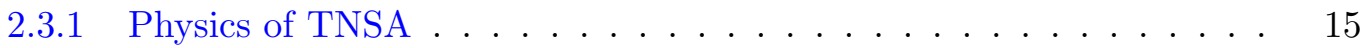

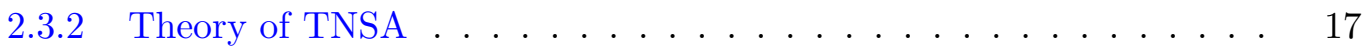

2.4 Radiation Pressure Acceleration . . . . . . . . . . . . . . . . . 23

2.4 .1 Comparison to TNSA . . . . . . . . . . . . . . . . 23

2.4 .2 Theory of RPA . . . . . . . . . . . . . 24

3 History of High Repetition Rate Ion Acceleration Schemes . . . . . . 29

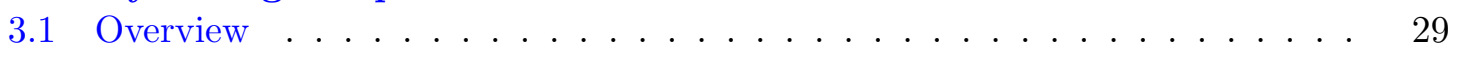

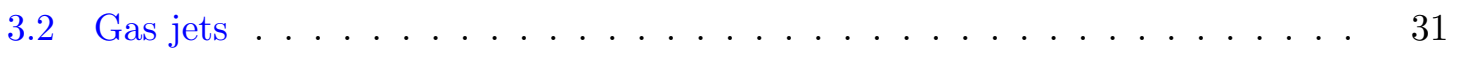

3.3 Tape targets . . . . . . . . . . . . . . . . . . . . . . 32

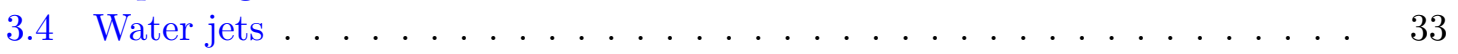


4 Design and Construction of Petawatt-Scale Scarlet Laser Facility . . . . 37

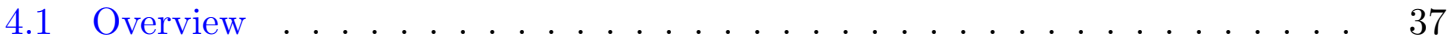

4.2 Introduction . . . . . . . . . . . . . . . . . 37

4.3 Laser design . . . . . . . . . . . . . . . . . . . . . . . . . . 38

4.3.1 Pulse generation and preamplification . . . . . . . . . . 38

4.3 .2 Amplification . . . . . . . . . . . . . . . . 40

4.3 .3 Compression . . . . . . . . . . . . . . . . . . 42

4.4 Diagnostics capabilities $\ldots \ldots \ldots \ldots \ldots \ldots \ldots$

4.4 .1 Laser diagnostics . . . . . . . . . . . . . . . . . . . . . . . . 45

4.4 .2 Target diagnostics . . . . . . . . . . . . . . . . . . . . . . 45

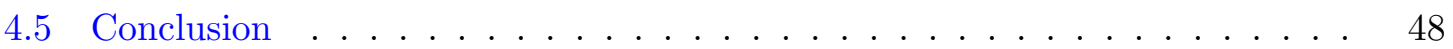

5 Damage Testing of Intense Laser Components . . . . . . . . . . . . . 50

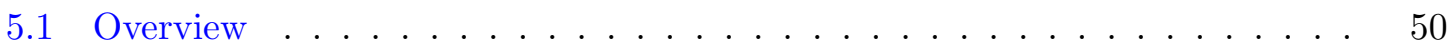

5.2 Introduction . . . . . . . . . . . . . . . 50

5.3 Grating fabrication . . . . . . . . . . . . . . . . 52

5.4 Experimental setup . . . . . . . . . . . . . . . . 53

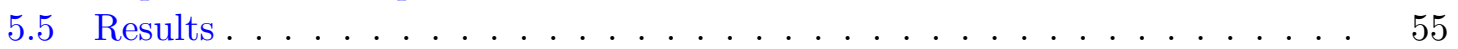

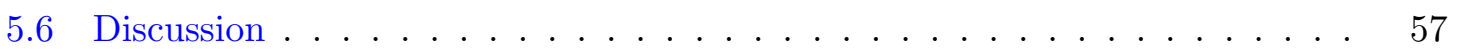

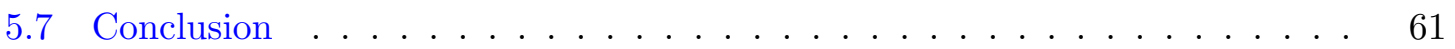

6 Liquid Crystals as Scientific Instruments for Intense Laser Applications 62

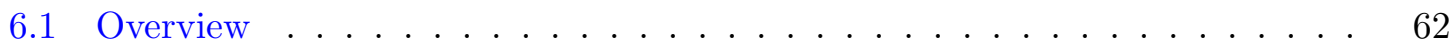

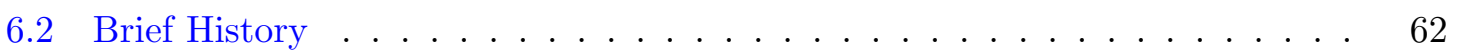

6.3 Relevant Chemistry . . . . . . . . . . . . . . . . 63

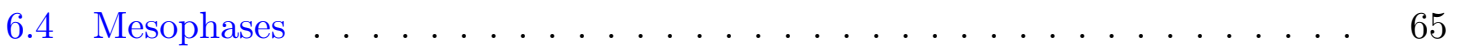

6.4 Nematic . . . . . . . . . . . . . . . 66

6.4 .2 Smectic . . . . . . . . . . . . . . . . 67

6.4 .3 Chiral . . . . . . . . . . . . . . . . 70

6.5 Freely Suspended Films . . . . . . . . . . . . . . . . . 72

7 Freely Suspended Liquid Crystals Film Formation Techniques . . . . . 75

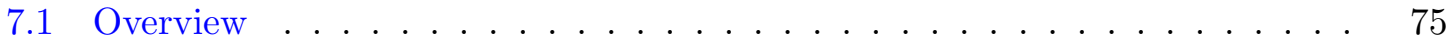

7.2 Previous methods of film formation . . . . . . . . . . . . 75

7.3 Thickness measurement of liquid crystal films . . . . . . . . . . . . . . . . 77

7.4 Thickness control of freely suspended films . . . . . . . . . . . . . . 81

7.4.1 Obstacles to uniform film thickness . . . . . . . . . . . . . . 81

7.4.2 Achieving uniform film thickness . . . . . . . . . . . . . . 83

7.5 Film frame design . . . . . . . . . . . . . . . 85

7.6 Film target formation . . . . . . . . . . . . . . . 87

7.7 Chariot and Rail film formation . . . . . . . . . . . . . . . 90

8 Single Shot Experiments on Liquid Crystal Targets . . . . . . . . . . . . 92

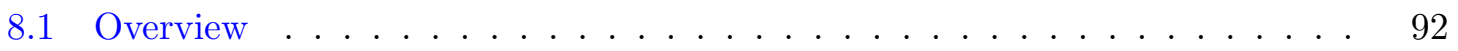

8.1 .1 Film formation . . . . . . . . . . . . . . . . . . . . . . . 92

8.2 Film alignment procedure . . . . . . . . . . . . . . . . . . . . . . . . . . . . . . .

8.3 Experimental results . . . . . . . . . . . . . . . . . 95 
9 Study of Ion Acceleration Mechanisms as a Function of Target Thickness Using Liquid Crystal Films . . . . . . . . . . . . . . . . . 99

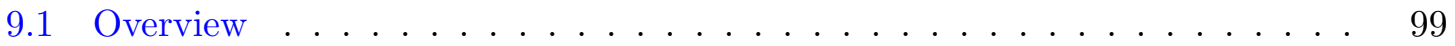

9.2 Prior literature on ion acceleration thickness scans . . . . . . . . . . . 99

9.3 Liquid crystal thickness scan setup . . . . . . . . . . . . . . . . . 100

9.4 Data . . . . . . . . . . . . . . . . . . . . . 104

9.5 Discussion . . . . . . . . . . . . . . . . . . . . . . . . . 109

9.6 Conclusion . . . . . . . . . . . . . . . . . . . . . 113

10 High Repetition Rate Liquid Crystal Film Target Inserter . . . . . . . . 114

10.1 Overview . . . . . . . . . . . . . . . . . . 114

10.2 Introduction . . . . . . . . . . . . . . . . . . . . 114

10.3 Linear Slide Target Inserter design . . . . . . . . . . . . . . . 115

10.4 Film control . . . . . . . . . . . . . . . . . . . . . . 118

10.4 Position repeatability . . . . . . . . . . . . . . 118

10.4.2 Thickness control . . . . . . . . . . . . . . . . . . 119

10.5 Conclusion . . . . . . . . . . . . . . . . . 123

11 FutureWork . . . . . . . . . . . . . . . . . . . . . . 124

11.1 Impact of Work . . . . . . . . . . . . . . . . . . . 124

11.2 Upcoming experiments . . . . . . . . . . . . . . . . . . . . . . . . . . . . . . . . . .

11.2.1 Film metallization . . . . . . . . . . . . . . . . . 124

11.2 .2 Laser pulse modification . . . . . . . . . . . . . . . . . . . 125

11.2.3 Relativistically Induced Transparency . . . . . . . . . . . . . . . . 127

11.2.4 Increased repetition rate for liquid crystal films . . . . . . . . . . . 128

11.3 Conclusion . . . . . . . . . . . . . . . . . . . 130

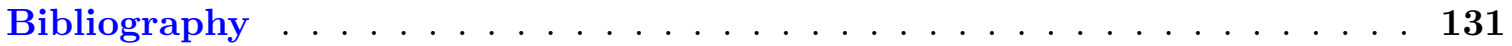




\section{List of Figures}

Figure

Page

1.1 Focused laser intensity as a function of time. The colored regions indicate different regimes of physics due to the fields an ionized electron will experience from the laser. The blue boxes on the right indicate the energies with which such an electron will be oscillated from the laser electric field. Adapted from [10]. . . . . . . . . . . . . . . . . . . . .

1.2 Neutron radiograph of an airplane jet turbine blade. The neutrons penetrate the metallic structure and scatter strongly from the internal defect filled with a remnant material from fabrication. Adapted from [12] . . . . . . . . . .

1.3 Graph of relative dose with respect to the depth of energy deposition into water (equivalent to human tissue) in $\mathrm{cm}$ for a few radiation and particle beams. Of these only protons exhibit a strong Bragg peak deposition which localizes dose to the area of a tumor with little damage to preceding tissue and virtually no damage beyond. Adapted from $[13] \ldots \ldots$. . . . . . . .

2.1 Cartoon showing the critical elements of the Target Normal Sheath-field Acceleration (TNSA) process. Here relativistic electrons accelerated from the front target surface by an incident laser leave the rear of the target, setting up a strong electric field between this accelerated electron cloud and the remaining positively charged target. This field accelerates charged particles from the back of the target, usually including contaminants inherent to $10^{-6}$ Torr vacuum chambers. . . . . . . . . . . . . . . .

2.2 Schematic for quasi-static theoretical model, wherein a plasma of electrons and immobile heavy ions occupies the half space $x<0 . \ldots \ldots$. . . .

2.3 Cartoon of the immobile heavy ion population (grey) and Boltzmann electron distribution (blue). The resulting charge separation creates an electric field directed away from the target. . . . . . . . . . . . . 
2.4 Cartoon depicting Radiation Pressure Acceleration (RPA). Each column is one step of the process, proceeding in time from left to right. The top row depicts laser intensity and electron and ion distribution profiles, while the bottom shows the location of those elements for each step. Initially the laser pushes the electron density forward before the ions have a chance to move (second column), creating an electric field in between these populations that will subsequently accelerate ions. At later times the ions are free to move as well, and for sufficiently long laser pulses there can be continued stages of electron density increase and ion acceleration. . . . . . . . . . . . .

3.1 Chart of laser shot rate in $\mathrm{Hz}$ as a function of time. Here a few key lasers are indicated, including the BELLA facility at Lawrence Berkeley National Laboratory (LBNL) and the HAPLS laser being constructed for the Extreme Light Infrastructure (ELI) project in Europe. . . . . . . . . . . . . . . .

3.2 Moving tape target concept schematic. Here two motors spin a thin strip of metal or plastic between two spools, leaving a region of vertically-moving planar target strip in between to be shot by the laser. From [54]. . . . . . .

3.3 Water jet ejected at speed from a nozzle, from [57]. This jet is tens of $\mu m$ in diameter, experiencing intense laser interaction from $1 \mathrm{kHz}$ pulses. . . . . .

4.1 Schematic of Scarlet pulse generation and amplification. Green boxes indicate pump lasers and their parameters, and gray boxes are the short pulse

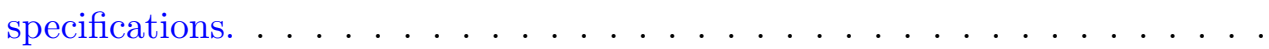

4.2 Shown here is the short pulse mode for each of the three passes within the final amplifier. Each short pulse pass and pump arm of the final amplifier can be monitored in real time with mode imaging cameras to ensure optimum pumping configuration and to protect from the development of hot spots within the beam mode. . . . . . . . . . . . . . . . . . . .

4.3 Small signal gain data for the final amplifier as the pump energy is increased up to its maximum of $50 \mathrm{~J}$ of $527 \mathrm{~nm}$ light. The curve is the theoretical small signal gain model, [40] to which the measured data conforms. . . . . .

4.4 Measurements of the short pulse spectrum at various amplification stages within Scarlet. The heights are not normalized, and vary only due to the proximity of the spectrometer fiber to the scatter surface during measurement. There is a shift toward longer wavelengths due to these being slightly preferentially pumped because the positive pulse chirp sends longer wavelengths through the fully energized crystals first. . . . . . . . . .

4.5 Schematic of the entire Scarlet system including the pulse generation and amplification and target areas. . . . . . . . . . . . .

4.6 Schematic of the Scarlet laser diagnostics table. This setup includes an autocorrelator for pulse width measurements, cross-correlator for contrast measurement, Spectralon reflection for mode quality diagnosis, as well as an energy meter and spectrometer for standard pulse characterization. . . . . .

4.7 The Scarlet focal spot, as observed at a low energy level on the focal spot alignment camera. The size is $<5 \mu m$ FWHM. . . . . . . . . . . . . 
4.8 An image of the Scarlet experimental chamber during a previous experiment. Various alignment and target diagnostics can be seen. The laser is incident on the target from the upper right region of the picture. . . . . . . . . .

5.1 (a) Scanning electron microscope (SEM) image of the conventional sputtercoated grating exhibiting a non-conformal groove structure that will decrease the diffraction efficiency. (b) SEM image showing the energetic sputtercoated grating with a more uniform groove structure, from Plymouth Grating Laboratory. . . . . . . . . . . . . . . . . . . .

5.2 (a) Schematic of vacuum chamber for damage testing. Of note are the autocorrelator to measure pulse width and the $10 \times$ microscope objective for in-situ damage verification. (b) Focal spot evolution and respective fit showing $M^{2}<1.2$. (c) Example in-situ damage image in false color. . . . .

5.3 Damage threshold data with curve fits for non-conformal (NC) and conformal (C) samples irradiated with $30 \mathrm{fs}$ pulses. For the Au-coated gratings, the conformal coating has a higher damage threshold than the non-conformal coating. Each data point represents 15 shots with a fluence error of \pm 0.02 $\mathrm{J} / \mathrm{cm}^{2} \ldots \ldots \ldots \ldots \ldots \ldots \ldots \ldots$

5.4 Pulse width dependence of damage threshold by sample. While the conformal (C) coating clearly has a higher damage threshold than the non-conformal (NC) coating, there is no clear pulse width dependence of the damage threshold observed for either grating type. Each data point represents 15 shots with a fluence error of $\pm 0.02 \mathrm{~J} / \mathrm{cm}^{2}$. . . . . . . . . . .

5.5 Autocorrelation trace of a $50 \mathrm{fs}$ pulse after compression by conformal $\mathrm{Au}$ gratings at the Scarlet laser facility. This initial measurement has since been surpassed by further grating optimization, allowing $30 \mathrm{fs}$ pulse compression to be achieved currently. . . . . . . . . . . . . . . .

5.6 SEM image showing intact fused silica substrate at a damage site on the conformal Au grating, produced from a $950 \mathrm{~mJ} / \mathrm{cm}^{2}$ pulse. . . . . . . . . .

5.7 (a) Shapes of the three different $\mathrm{Au}$ surfaces corresponding to differentlyconforming Au layers. (b)-(d) Color-coded magnetic field enhancement $\left|H / H_{0}\right|$ for the gratings C1-C3, respectively, centered around one diffraction ridge (visible at the bottom of each image). Here $H_{0}$ is the magnetic field of the p-polarized light wave with $\lambda=800 \mathrm{~nm}$, incident at an angle $\theta=46^{\circ}$. The max value of $\left|H / H_{0}\right|$ is indicated above each color bar, and decreases for the more conformal curvatures. Other wavelengths within the experimental range were also simulated, with similar field enhancement results to those shown here. . . . . . . . . . . . . . . . . . . . .

6.1 Chemical formula of the liquid crystal 4-cyano-4'-octylbiphenyl (8CB), along with its mesophases and their transition temperatures. . . . . . . . .

6.2 Cartoon of two common liquid crystal mesophases: nematic, which exhibits only orientational order, and smectic, which has orientational and positional order. . . . . . . . . . . . . . . . . . 
6.3 Cartoon of a smectic phase liquid crystal droplet placed onto a substrate. Rather than spreading out as a water droplet might, the volume will spread into stacks with points of rapidly varying thickness that resemble solid material cleaves. . . . . . . . . . . . . . . . . .

6.4 Cholesteric or chiral liquid crystal phase. Here each smectic layer has a different director orientation, and the pattern repeats itself after the pitch distance $p \ldots \ldots \ldots \ldots \ldots \ldots \ldots \ldots \ldots$

6.5 A 8CB film formed within a $6 \mathrm{~mm}$ inner diameter washer. Initially there was a vertical thickness gradient from excess volume (left), but once this excess wicked away the annealed film remained that thickness $(300 \mathrm{~nm})$ more than a year later (right) . . . . . . . . . . . . . . . . . .

7.1 Due to the beneficial surface tension of the smectic phase, liquid crystals can be formed simply by wiping a small volume across an aperture in a rigid frame, as depicted here. Volumes as low as $100 n L$ are sufficient to make films in apertures of diameter between a few $m m$ several $\mathrm{cm}$. The wiper can be any non-absorbing material with a flat edge to ensure uniform contact with the frame. . . . . . . . . . . . . . . . .

7.2 Four different volumes applied next to $4 \mathrm{~mm}$ apertures in a copper frame. These volumes were metered out by a precision syringe pump with a $25 \mu \mathrm{L}$ syringe. In general more volume results in a thicker film, with $0.1 \mu L$ or less being sufficient for film formation. . . . . . . . . . . .

7.3 Chart showing the reflected color from a thin $8 \mathrm{CB}$ film with respect to thickness. Sub-50 $\mathrm{nm}$ films are black films, where all visible wavelengths destructively interfere upon reflection. Increasing thickness from these dark films leads to a series of color bands, eventually resulting in primarily pink and green bands due to the combination of colors interfering constructively at those large thicknesses. Image credit RJ Hanna. . . . . . . . . . . . . . .

7.4 Fringes visible in the reflection of a $532 \mathrm{~nm}$ laser with $F / 1$ focusing on an $11 \mu \mathrm{m}$ shard of transparent mica. The fringe number can be correlated to a thickness value once the spread of incident light angles has been determined from the focusing parameters. . . . . . . . . . . . . . . .

7.5 Spectral reflectance technique utilized by the Filmetrics thickness measurement device. The graph at the right shows the modulations in the reflected white light (blue) and the results of an iterative algorithm to match this curve based on an initial thickness guess (red). . . . . . . . . . . . .

7.6 $4 \times$ magnification image of one section of a $4 \mathrm{~mm} 8 \mathrm{CB}$ film. Visible is a thin film region in the lower right, a ring of thicker film, and then the opaque meniscus region. Each of these regions is comprised solely of the liquid crystal $8 \mathrm{CB}$, and their varying appearance comes from the changes in thickness and molecular orientation. . . . . . . . . . . . . . . . 
7.7 Here a so-called "amphitheater film" is shown, where the thickness decreases in radial steps from the outer to inner ring. Depicted are both a) a cartoon of this phenomenon and b) an actual amphitheater film within a $4 \mathrm{~mm}$ aperture. These rings are different in thickness by many layers, giving rise to the distinct bands observed here. It is possible for the thickness range of such a film to span from a few $\mu \mathrm{m}$ at the outer ring to a sub-50 $\mathrm{nm}$ film in the center. . .

7.8 Film formed within a frame held vertically. The excess volume of liquid crystal is dragged down by gravity such that the thickness of the film increases lower down within the aperture, as can be seen by the bands of color variation. 83

7.9 A region of thicker liquid crystal floating upon the thin substrate, known as an island, can be seen here. Islands are typically a few $\mathrm{mm}$ across and move around on the overall film surface due to air currents or gravity. The right hand image actually has two island regions forming a tiered thickness area in the center of the film, visible as strong and faint white reflection regions.

7.10 Temperature control is required for precise thickness manipulation. This copper frame is $6 \mathrm{~mm}$ thick, allowing inserting of three $3 \mathrm{~mm}$ resistive cartridge heaters to be inserted into the bulk metal, visible at the bottom of the image. A thermocouple can be seen at the top of the frame for temperature monitoring and for control through a Proportional Integral Derivative (PID) controller. . . . . . . . . . . . . . . .

7.11 Frames for film formation were made with various aperture thicknesses as well as a number of diameters, two of which are shown on this frame. . . . .

7.12 Diamond edge frames for precise longitudinal film formation positioning. a) shows a schematic of a $10 \mu \mathrm{m}$ diamond edge grown within the silicon aperture. b) shows the diamond edges as they are bought commercially, and c) indicates how they were implemented on one of the copper frames described above. A liquid crystal film can be seen within the diamond edge aperture on this last image. . . . . . . . . . . . . . . . . . .

7.13 Example film formed within a $4 \mathrm{~mm}$ diameter aperture with poor edge quality. Films are more difficult to form in such frames, and have poor flatness and exacerbated thickness nonuniformity. . . . . . . . . . .

7.14 Example image of the flat frame set on the target positioner with the Scarlet experimental chamber. This frame holds four possible targets of $4 \mathrm{~mm}$ diameter, and can be heated with a resistive pad outside the chamber to form the desired thickness film. . . . . . . . . . . . . .

7.15 Individual copper flags with $4 \mathrm{~mm}$ diameter apertures for film formation prior to shot time. These flags are set in a copper block connected to the resistive heaters and PID temperature control system and can be aligned using a similar block on the target positioner within the chamber. At right are many flag targets with films ranging in thickness from a few-hundred $\mathrm{nm}$ to a few $\mu \mathrm{m}$. Their resilience allows these films to sit for weeks and longer without changing thickness if left in an environment with modestly regulated temperature. . . . . . . . . . . . . . . . 
7.16 The chariot and rail film formation device. Two interlocking copper pieces a) can be heated via resistive cartridge heaters described previously. Liquid crystal applied to the edge between the two pieces will be drawn into a film as they are moved apart. Films several $\mathrm{cm}$ wide are possible with this device. Rapidly stretching a film in this device b) will cause multiple thickness regions of different color to appear. . . . . . . . . . . . . . . . . .

8.1 Example film thicknesses with measurement from the Filmetrics multispectral interferometer demonstrating the thickness range of liquid crystal targets. The plots show reflectance vs. wavelength where the blue curve is the measured reflectance from the film and the red curve is the result of an iterative calculation to match this spectrum. In general, more peaks correspond to a thicker film. The images to the left of each plot show an example film within a copper frame at each of these thicknesses. . . . . . .

8.2 a) Cartoon of shadowgraphy alignment onto focal spot camera using $3 \mu \mathrm{m}$ aluminum fiducial floated on a liquid crystal film. b) The fiducial would exacerbate any thickness non-uniformities, but c) careful film formation allowed uniform thickness films to have aluminum fiducials placed within. .

8.3 $3 \mu \mathrm{m}$ thick aluminum shard floated within edge of $4 \mathrm{~mm}$ liquid crystal frame as viewed from a high field of view telescope Questar system. The left image shows a floated fiducial within a film, while the right image was taken after the laser shot once the film was gone-note the slight change in reflected light within the film area. . . . . . . . . . . . . . . . . .

8.4 a) Schematic of the Scarlet laser main experimental target chamber. A F/2.2 Off-Axis Parabola (OAP) sends the 5.5" diameter beam toward the copper target frame shown in the left inset. An in-vacuum camera situated behind the target along the laser axis is used for alignment, while a Thomson parabola spectrometer collects ion data along the rear-targetnormal direction. b) The actual experimental setup with OAP in the background and the target frame and alignment objective in the foreground. c) Focal spot alignment camera shadowgraphy image of alignment light striking the aluminum fiducial floating on a liquid crystal film. . . . . . . .

8.5 Ion acceleration data from the Thomson parabola spectrometer. a) The accelerated ions from a $100 \mathrm{~nm} \mathrm{Si}_{3} \mathrm{~N}_{4}$ solid target are shown as well as b) the traces from a $700 \mathrm{~nm}$ thick liquid crystal film target, and c) from a 160 $n m$ film. Both liquid crystal ion traces show a strong proton signal with max energy around $10 \mathrm{MeV}$, as well as multiple traces from other ion species, and are comparable to the trace from the similar-thickness solid target. . . . . .

9.1 a) The Confocal High Intensity Positioner (CHIP) is designed in a cagemount system for compactness and rigidity to ensure its precision. b) A vertical translation stage allows the input optics to be lowered to safety during a laser shot. c) A motorized stage allows the CHIP to be finely aligned along the front-side target normal direction. d) Cartoon of the target handoff procedure including shadowgraphy of the copper nanoparticle coated slide. e) Diagram of the mismatch in actual and apparent focal plane of the focal spot alignment camera due to light traveling through the handoff target.102 
9.2 Cartoon of experimental chamber for thickness scan run. Of note are the CHIP and focal spot camera for alignment, the Filmetrics for target thickness measurement, and the Thomson parabola and two radiochromic film (RCF) stacks for accelerated ion measurement. . . . . . . . . . . . . . . .

9.3 a) Spatial orientation of the two RCF stacks. The one on the right (looking along the laser $k$-vector) had a $4 \mathrm{~mm}$ hole punched to allow ions to travel directly to the Thomson parabola beyond. b) Each RCF stack was comprised of six pieces of film with appropriate filtering to observe proton energies indicated here in white. The layout of the films shown here will be replicated for all subsequent RCF images presented. The black numbers in the lower right of each layer will be visible as well. c) Graph showing the deposited energy into each layer of the RCF stack. Higher energy protons travel through the initial stacks with relatively little dose and deposit most of their energy within a subsequent stack once their speed has been reduced by collisions in the previous layers. . . . . . . . . . . . . . . . .

9.4 Maximum proton energies recorded along the target normal direction by the Thomson parabola spectrometer for various thicknesses of liquid crystal films. Also plotted are two $2 \mu \mathrm{m} \mathrm{Cu}$ foils and one $\mathrm{Si}_{3} \mathrm{~N}_{4} 100 \mathrm{~nm}$ membrane for comparison. There is a broad peak observed in the 600-900 $\mathrm{nm}$ range, suggesting an optimization of TNSA here. . . . . . . . . . .

9.5 RCF data from a $640 \mathrm{~nm} 8 \mathrm{CB}$ film. The laser-axis stack has more dose (i.e. darker blue films) than the target normal sheet, which clearly indicates a non-TNSA acceleration mechanism. The shadow of a rectangular piece of CR39 with holes drilled in it can be seen in the laser-axis sheets as well-this essentially acted as an extra filter, raising the energy of protons necessary to reach each of these sheets. . . . . . . . . . . . . . . .

9.6 a) Spatial location of the two RCF packs with an overlay demonstrating the radial integration process for angular dose signal collection. Zero degrees is set to be the target normal direction. b) Graph of deposited energy density as a function of angle away from the target normal direction for all six film stacks on a shot of $640 \mathrm{~nm} 8 \mathrm{CB}$. c) Angular data from layers 3-6, where an increasing dose as a function of angle appears. This behavior is indicative of an ion acceleration mechanism other than TNSA. . . . . . . . . . . .

9.7 Angular dose data from two different liquid crystal shots. The left chart is of a $715 \mathrm{~nm}$ film that exhibits a sharp decline in signal away from the target normal direction, indicative dominant TNSA acceleration. The data on the right is from a $610 \mathrm{~nm}$ film with a slighter slope, suggesting a contribution from a non-TNSA mechanism at this thickness. . . . . . . . . . . .

9.8 RCF stacks demonstrating the low-energy annular dose ring resulting from liquid crystal shots. This was not observed on the $\mathrm{Cu}$ and $\mathrm{Si}_{3} \mathrm{~N}_{4}$ targets taken during this run. . . . . . . . . . . . . . 
9.9 Thomson parabola trace for a shot that exhibited some energy bunching. The graphs are lineouts of some of the individual ion traces for different ionization states of carbon. Here sharp jumps can be observed in the cutoff for one species compared to the turn-on of the next. This can also be seen in the raw image scan at left, where the brightness changes sharply horizontally across ion species. Typical TNSA spectra do not exhibit these bunching features, suggesting another mechanism is contributing here. . . . . . . .

10.1 Schematic of Linear Slide Target Inserter (LSTI). A vacuum-compatible motor moves a wiper vertically with variable speed to form a film within the $4 \mathrm{~mm}$ diameter aperture. Horizontal channels allow for temperature change via a chiller water line and monitoring via type $\mathrm{K}$ thermocouple. The clearance hole in the wiper allows for additional liquid crystal volume deposition. . . . . . . . . . . . . . . . .

$10.24 \mathrm{~mm}$ diameter films made with the LSTI to demonstrate fine thickness control. Shown are four films separated in thickness enough to show different colors, though finer thickness gradations are possible with this film formation device. . . . . . . . . . . . . . . .

10.3 The geometry of the beveled aperture and raised wiper provide a consistent film formation plane. a) A film initially forms between the aperture and the wiper, such that it is being pulled at an angle with respect to the aperture plane. b) As the wiper continues drawing, more of the film is transferred to front edge of the beveled aperture. c) Snapshots taken during film formation. As the wiper moves left to right the forming film is brought closer to co-planar with the frame aperture, resulting in the correct angle for observing the pink color stemming from constructive interferences at this film thickness of 530

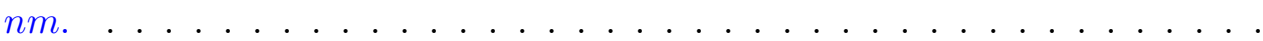

10.4 Control of film thickness via LSTI wiper speed. Here the vertical bars show the range of films formed over five draws at each speed, while the dots indicate the average of those films. This average increases in thickness as the wiper speed decreases. The shaded areas indicate three different regions of thickness range, discussed in the text. . . . . . . . . . . . . . . . .

$11.1500 \mu \mathrm{m}$ of copper ions sputter coated onto a $4 \mathrm{~mm}$ diameter liquid crystal film. The result is a black, uneven surface within the frame aperture, but evidence that the liquid crystal film survived long enough to act as a substrate for the metal ions. . . . . . . . . . . . . . . . . .

11.2 Left: Plasma mirror concept using liquid crystals. Here the yellow film is transparent to the prepulse but becomes reflective once the main pulse creates a critical density plasma on it. Right: Plasma filter concept, wherein the prepulse is absorbed or reflected by the yellow film, and the main pulse pushes through this reduced density to the main target. . . . . . . . . .

11.3 Left: Possible chamber setup for experiment investigating relativistically induced transparency (RIT), where the transmitted signal is observed on a Spectralon screen as a function of incident laser intensity. Right: Schematic graph of transmitted signal vs. laser intensity, with a large signal increase indicating the onset of RIT . . . . . . . . . . . . . . . . . . 
11.4 Spinning disk target inserter for $1 \mathrm{~Hz}$ and faster film formation currently under development. The device consists of a stationary Teflon blade with a spinning aluminum disk where films are formed underneath. . . . . . . . . .

11.5 Microfluidic jet cartoon and microscope image. Here a deionized water jet is pushed through a microfluidic channel with a height of $30 \mu \mathrm{m}$ and width of $1 \mathrm{~mm}$. The resulting planar jet region is on the order of $1 \mathrm{~mm}$ as well, and has thickness roughly matching that of the channel height. Microfluidic channels made by Mike Prikockis of the R. Sooryakumar group at Ohio State.130 


\section{List of Tables}

Table

Page

1.1 Requirements for high repetition rate targetry. . . . . . . . . . . . 7

4.1 Beam parameters available in the different shot modes of Scarlet. . . . . . . 46

5.1 Single-shot damage thresholds ( $0 \%$ damage probability) in $\mathrm{mJ} / \mathrm{cm}^{2}$ for three gratings tested at multiple pulse widths. . . . . . . . . . . .

5.2 Measurements and simulation calculations of the absorptivity A, negative first diffraction order reflectivity $R_{-1}$, and specular reflectivity $R_{0}$ for three different grating profiles (with C3 being the most conformal), as well as measured laser-damage threshold (LDT) values for the relevant profiles . .

6.1 Phase transitions of cyanobiphenyl liquid crystals. The listed value is the temperature at which transition from the phase in that column occurs. $\mathrm{C}$ is crystalline, $\mathrm{S}_{A}$ is smectic $\mathrm{A}, \mathrm{N}$ is nematic, and $\mathrm{I}$ is isotropic (liquid) phase. Modified from $[109] \ldots \ldots \ldots \ldots \ldots$ 


\section{Chapter 1 INTRODUCTION}

\subsection{High Energy Density Physics}

The laser has continued to prove itself an unparalleled tool for scientific investigation since its invention in 1960. Controlled coherent radiation has applications throughout physics for measurement and diagnostic purposes, and to create and probe states of matter for their underlying physical properties.

Today the most powerful laser facilities routinely reach intensities of $10^{21} \mathrm{~W} / \mathrm{cm}^{2}$, which is a greater power density than all the sun's output that strikes the Earth concentrated into an area the size of a human hair. This immense intensity is commonly achieved with incredibly short laser pulse durations, on the order of tens of femtoseconds $\left(10^{-15} s\right)$. Facilities that can generate such pulses range from the gargantuan-the National Ignition Facility (NIF) at Lawrence Livermore National Laboratory (LLNL) has a footprint larger than three football fields-to the table-top size, as can be found in a number of university laboratories. This science is collectively called High Energy Density Physics (HEDP) due to the temperatures (millions of kelvins) and pressures ( $>1 \mathrm{Mbar}$ ) produced by these lasers.

The potential applications of ultra-intense lasers are manifold, ranging from energy deposition into a compressed fuel pellet during fusion ignition[1] to laser wakefield acceleration[2] to ion acceleration and its applications of neutron radiography,[3] nuclear physics,[4] and proton cancer therapy.[5] Furthermore, an understanding of energetic electrons produced during these laser interactions leads to efficient x-ray generation[6, 7] and positron production, [8].

A relatively new but exciting application is found in laboratory astrophysics.[9] This takes advantage of the laser's ability to generate temperature and pressure conditions equivalent to those in the sun, allowing those states of matter to be probed in the laboratory. These sorts of high pressure or high temperature experiments reveal information on astrophysical phenomena from the nature of photoabsorption in the sun to the consistency of the core of Jupiter. 


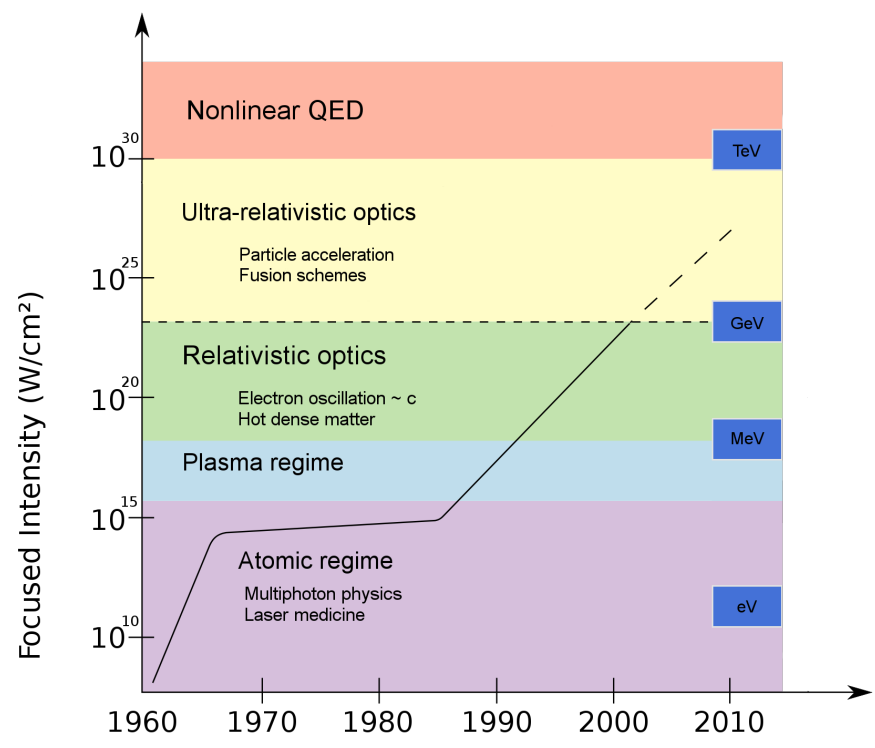

Figure 1.1: Focused laser intensity as a function of time. The colored regions indicate different regimes of physics due to the fields an ionized electron will experience from the laser. The blue boxes on the right indicate the energies with which such an electron will be oscillated from the laser electric field. Adapted from [10].

Figure 1.1 shows the increase in laser intensity over the past several decades, along with the regimes of physics this tool has unlocked. The blue boxes on the right of the graph indicate the energy an electron will gain as a result of its oscillation from the laser electric field. Current facilities can achieve $10^{21} \mathrm{~W} / \mathrm{cm}^{2}$ and above, where electrons ionized by these fields gain relativistic energies within one optical cycle of the laser.

\subsection{Applications}

The field of HEDP grew out of a search for fusion energy decades ago. The idea is to use the incredible pressures and temperatures possible with intense lasers to compress a fuel pellet until the onset of fusion, releasing energy. This is known as Inertial Confinement Fusion (ICF). To this end a number of large scale facilities have been developed, including the largest one: the NIF at LLNL, which compresses a deuterium-tritium fuel pellet using 192 separate but synchronized laser beams. Sufficiently uniform compression using this method has proven difficult, and so an alternative has been investigated where an ultra-short pulse impacts the target during an intermediate compression stage to bring the core of the fuel pellet to the appropriate conditions for fusion. This technique has come to be known as Fast Ignition (FI), and has been the subject of considerable study for some time.[1]

As more facilities were developed to study the fusion problem, other applications for 
this sort of research naturally arose. First developed to study similar fundamental science, many of these mid-level facilities now focus exclusively on applications entirely separate from the fusion problem. One significant benefit the smaller facilities have is the ability to fire much faster than their large counterparts, enabling a broader range of research topics to be investigated. A few of these applications are outlined below, with an emphasis on those that benefit especially or outright require high repetition rate facilities.

\subsubsection{Energetic beam production}

One of the widest areas of application for intense beam interaction with a target lies in energetic beam production. This can come in many forms-x-rays, gamma rays, ion beams, electron beams-each of which can be optimized or selected for with different laser and target characteristics. Collectively these phenomena are sometimes called secondary radiation.

Broadly speaking, secondary radiation sources have experimental interest as tools to probe other states of matter and as applications directly. As such the fundamental interactions producing secondary radiation are of interest. For example, proton beams generated from the laser-matter interaction (via mechanisms to be discussed later) can be used to probe otherwise opaque experimental setups.[11] This proton radiography technique is particularly useful for probing a fusion target during compression in order to view instabilities at different stages during that process.

As another example, a beam of neutrons can be produced with lasers.[3] Proton or deuteron beams generated from the initial laser-target interaction can be directed into a converter target, such as lithium or beryllium, in which a nuclear reaction producing neutrons can occur. The optimization of such a neutron beam depends on control of the yield and energy spectrum of the laser-generated ion beam as well as the details of the converter target.

One application of such a neutron source is non-destructive evaluation (NDE), where an object is scanned to ascertain its contents or properties without causing damage. For example, the low mass of the neutron compared to heavy nuclei allows them to scatter through dense materials unhindered. This allows neutron beams to penetrate where $\mathrm{x}-$ rays cannot, for example through lead. Such a beam could be used to identify materials within a protected enclosure, e.g. scanning containers at a freight checkpoint. Additionally, neutrons will produce a characteristic gamma ray signal when they interact with fissile material, enabling the detection of these even if they are being concealed.

Neutron beams have also been used to detect internal defects in metallic airplane parts using neutrons produced from a nuclear reactor. Figure 1.2 shows a neutron radiograph of a jet turbine blade. These and many other airplane parts are typically cast in ceramic molds, and improper fabrication can leave internal pockets of this material inside the metallic part. Such defects will be invisible to visual inspection and x-ray probing, but not to neutrons 


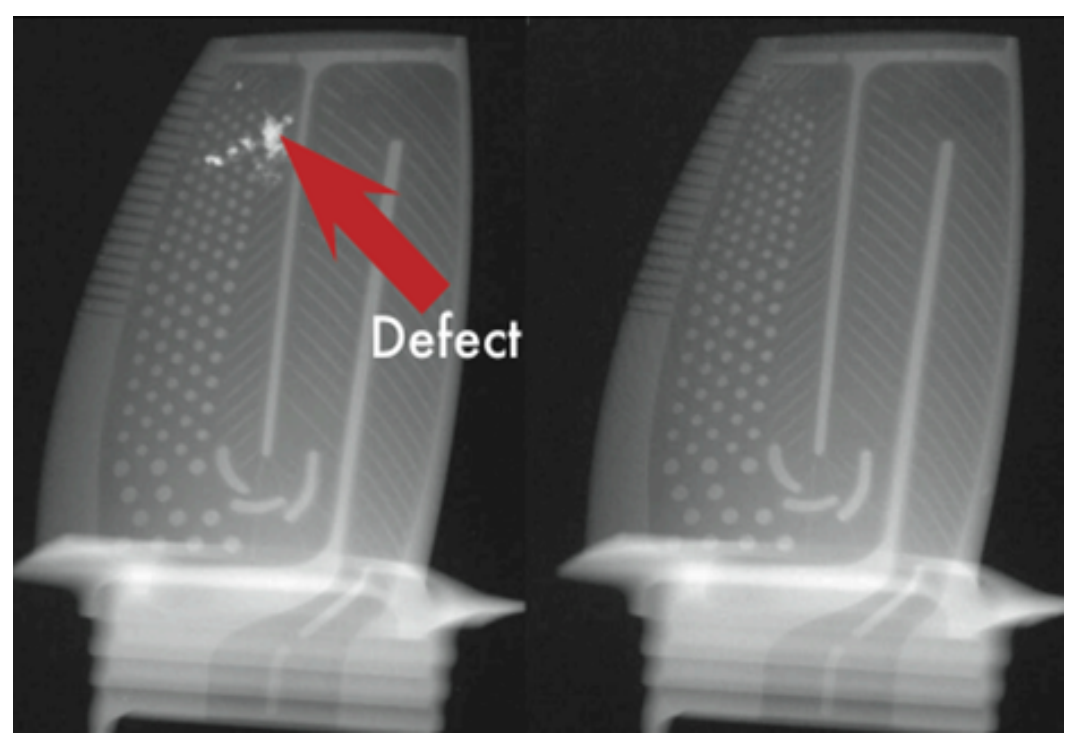

Figure 1.2: Neutron radiograph of an airplane jet turbine blade. The neutrons penetrate the metallic structure and scatter strongly from the internal defect filled with a remnant material from fabrication. Adapted from [12].

due to their scattering properties. A laser-based neutron source would have advantages in operation cost and is potentially portable, as the technology exists to make the requisite laser for this application on the back of a semi-trailer truck. To achieve appropriate neutron flux, however, the laser would need to fire at a planar target at repetition rates on the order of $10 \mathrm{~Hz}$.

\subsubsection{Hadron cancer therapy}

One of the most appealing HEDP applications is the promise of ion beam cancer therapy. This technique relies on the specific type of scattering that charged baryonic particles experience as they enter a material. For example, protons typically lose energy via Coulomb interactions with outer-shell electrons in the material they are traveling through. Exciting and ionizing these atoms takes relatively little energy from the proton, so that an accurate approximation to assume is that the proton is continuously slowing down as it travels deeper in the material, losing energy to surrounding atoms. The secondary radiation produced from the proton collisions is locally absorbed within $<1 \mathrm{~mm}$, and there is no significant deflection of the proton as it travels.

This proceeds until the particle slows down enough that the cross section for interaction (which scales as the velocity $v^{-2}$ ) reaches a significant value, at which point the particle stops and deposits all its remaining energy at once. This is the familiar Bragg peak for ion stopping in material. This Bragg peak deposition behavior makes a proton beam promising 


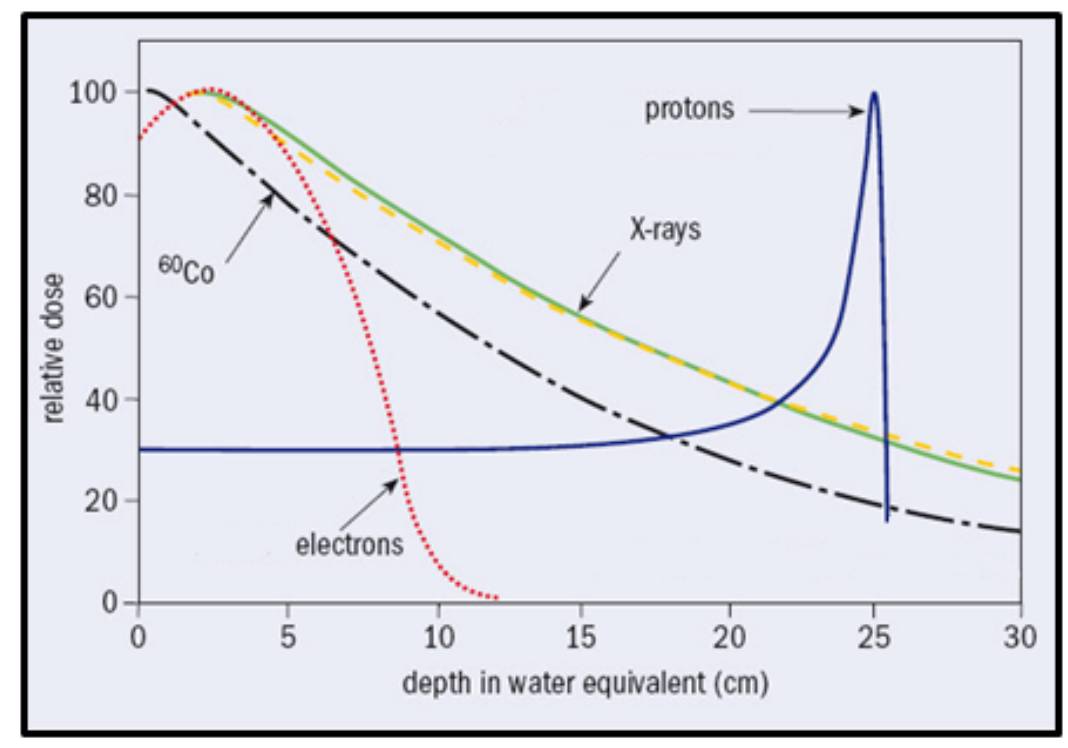

Figure 1.3: Graph of relative dose with respect to the depth of energy deposition into water (equivalent to human tissue) in $\mathrm{cm}$ for a few radiation and particle beams. Of these only protons exhibit a strong Bragg peak deposition which localizes dose to the area of a tumor with little damage to preceding tissue and virtually no damage beyond. Adapted from [13].

compared to x-rays or other electromagnetic radiation, which will be much more damaging to the surrounding tissue once sufficient intensity is achieved to destroy an internal tumor. Relatively little dose is applied prior to the Bragg peak location, and virtually no energy is deposited after this point, as can be seen in Fig. 1.3.

The depth of Bragg deposition depends on the energy of the incident particle. The problem of hadron cancer therapy, therefore, lies in the generation of a beam of particles of known and easily-tunable energy distribution. Much research on ion acceleration today focuses on understanding the physics behind ion acceleration mechanisms in order to learn how to better control and tune them for applications like this one.

Accelerator facilities have been built to date to generate such a beam of protons, although the initial and operating costs are prohibitive. One of the largest single expenses is the gantry system, often weighing in excess of 100 tons, which must be spun around a patient as a single unit to provide a precise, directed radiation dose to the targeted area. This device must include a central accelerator, beam transport channels, and a magnetic system in the gantry all to produce and target the proton beam.[5] Rotating this entire assembly around the patient significantly increases the operating costs, and therefore decreases the number of facilities that can afford to build such a device.

A laser-based proton acceleration mechanism would remove much of these constraints, since the device would now consist of a high intensity laser which simply needs to be aimed 
appropriately at a target such that the resulting particle beam travels along the correct path into the patient. From an engineering perspective this significantly decrease the size and therefore cost of the gantry system. Additionally, one laser system could serve a number of such gantries, again reducing the cost and increasing total patient throughput.

A number of obstacles have presented themselves with regards to this application. First, 230-250 $\mathrm{MeV}$ energy protons are necessary for deposition $20 \mathrm{~cm}$ into tissue, a number that has been difficult to reach simply by scaling up existing systems because the fundamental physics of laser acceleration mechanisms are still not fully understood. Second, yields of $\sim 1 \times 10^{10}$ protons $/ \mathrm{s}$ are required, which necessitates $\sim 10 \mathrm{~Hz}$ repetition rate laser shots. The first facilities that can operate at these rates have been commissioned recently and more are coming online in the next several years, but as will be discussed in this work there is not yet a satisfactory high repetition rate target delivery mechanism. Lastly, the beams need to be sufficiently monoenergetic that their deposition can be localized. Current implementations of laser-ion acceleration mechanisms result in broad energy spectra-these could be filtered, but at the cost of requiring even higher repetition rates to make up for the lost proton yield at the desired energies.

\subsection{High repetition rate lasers}

The promise of unlocking new applications and of performing experiments more thoroughly and quickly has pushed progress towards higher repetition rate laser systems. The first large laser systems were limited to one shot every several hours because of the cooling time necessary to prevent damage and adverse lasing conditions in the amplifier gain media. As technology has improved, ultra-intense laser repetition rates have significantly increased: currently operating systems with hundreds of $T W$ firing on the $0.1 \mathrm{~Hz}$ timescale are common, and there are a handful of $P W$ facilities with repetition rates approaching 1 $H z$. These numbers will all be increasing in the near future: as part of the European Extreme Light Infrastructure (ELI) project, a total of $€ 1$ billion is being spent on three cutting edge laser facilities, each with multiple laser systems approaching the several $P W$ at $10 \mathrm{~Hz}$ scale.

While significant effort has gone into the development of laser technology to produce intense pulses at high repetition rates, less emphasis has been placed on the capability to deliver satisfactory targets at these speeds. Table 1.1 lists a number of parameters that are desired for such a target system. The target composition must be proton or deuteron rich, depending on the desired ion acceleration mechanism and ultimate application. Thickness tuning provides both acceleration mechanism selection and optimization of that mechanism that depends on the laser energy, pulse duration, and focusing geometry being used. Positioning is critical since there is no time for realignment between shots, a task that 
Table 1.1: Requirements for high repetition rate targetry.

\begin{tabular}{|c|c|c|}
\hline Parameter & Requirement & Operational Impact \\
\hline Composition & $\mathrm{P}$ or $\mathrm{D}$ rich & Required for ion acceleration approaches \\
\hline Thickness-coarse & $\sim 200 \mathrm{~nm}$ to $\sim 2 \mu \mathrm{m}$ & Selects acceleration mechanism \\
\hline Thickness-fine & Factors of 2 & Tune target thickness to laser parameters \\
\hline $\begin{array}{l}\text { Positioning-- } \\
\text { longitudinal }\end{array}$ & $1 \mu m$ & $\begin{array}{l}\text { Required for consistent intensity on tight } \\
\text { focusing geometry }\end{array}$ \\
\hline $\begin{array}{l}\text { Positioning- } \\
\text { transverse }\end{array}$ & $\begin{array}{l}\sim 100 \mu m \text { transverse ex- } \\
\text { tent }\end{array}$ & $\begin{array}{l}\text { Must accommodate beam pointing varia- } \\
\text { tion }\end{array}$ \\
\hline Insertion rate & $1 /$ minute to $>10 \mathrm{~Hz}$ & $\begin{array}{l}\text { To serve existing/upcoming lasers and } \\
\text { applications }\end{array}$ \\
\hline Target separation & Dependent on design & Collateral damage tolerance \\
\hline Pressure & $<10^{-6}$ Torr & High vacuum required for experiment \\
\hline Temperature & Room temperature desired & Cryogenics are significant complication \\
\hline Cost & $\ll \$ 1$ target & $\begin{array}{l}\text { Scan may take several hours: } \$ 1 / \text { shot at } \\
10 \mathrm{~Hz} \text { is } \$ 36,000 / \text { hour }\end{array}$ \\
\hline
\end{tabular}

currently takes on the order of half an hour due to the $\mu m$ precision required along the incoming laser direction to ensure optimum focused intensity on target. The insertion rate for the applications listed above and that is required for the new laser facilities can be up to $10 \mathrm{~Hz}$. All of this must be done under the high vacuum necessary for short pulse propagation to a target, and preferably at standard target chamber temperatures. Finally, the cost of such targets is a major concern: typical HEDP targets can be upwards of $\$ 100$, and even reducing this by a few orders of magnitude can lead to considerable operational costs.

\subsection{This Work}

What follows is a description of work toward developing a superior target for HEDP and related experiments. The adaptation of liquid crystals into variable thickness films provides the opportunity to quickly select and optimize ion acceleration mechanisms, and the ability to form such films rapidly enables a number of high repetition rate laser experiments and applications. These two advancements together represent significant progress for intense laser science.

This thesis is arranged as follows:

Chapter 2 is an introduction to the two most relevant ion acceleration mechanisms for the currently performed liquid crystal experiments. This includes a theoretical overview of the 
initial laser-matter interaction, the physics and theoretical model for Target Normal Sheathfield Acceleration (TNSA), followed by the same for Radiation Pressure Acceleration (RPA).

Chapter 3 outlines progress previous to this work on creating a high repetition rate target. This includes gas and liquid droplet jets, solid tape targets, and liquid streams. The relevant positive and negative aspects of these approaches will be detailed.

Chapter 4 is an overview of the Scarlet laser facility, which is an ultra-intense short pulse laser system at The Ohio State University. The repetition rate of the laser is once per minute-much faster than large scale facilities, but already slower than recently completed and upcoming systems. Scarlet was recently upgraded to better enable high repetition rate ultra-intense experimentation on fundamental science related to the applications described above.

Chapter 5 details work on the damage testing of optics for the Scarlet upgrade. Though such experimental testing is necessary to ensure the safe operation of a high repetition rate system like Scarlet, the fundamental science behind the damage mechanism is of independent interest as well.

Chapter 6 is a theoretical introduction to the physics of liquid crystals. The phases most relevant to this work will be listed and detailed, as well as previous work forming freely suspended liquid crystal films for fluid dynamics experiments.

Chapter 7 is a practical explanation of the formation and thickness control of liquid crystal films. As will be explained, making a film with liquid crystal is straightforward, but controlling the thickness of that film is not. The details of film formation will be described, as well as a number of devices made for this purpose.

Chapter 8 describes the initial experiment performed on freely suspended liquid crystal films at the Scarlet laser facility. The purpose here was ion acceleration from films made one at a time, or in single-shot mode.

Chapter 9 includes a further experiment where the thickness of the liquid crystal films was tuned as the ion acceleration from different mechanisms (e.g. TNSA and RPA) was monitored. This reveals an optimization of TNSA at a certain thickness, along with evidence of a different mechanism and other results that will be discussed.

Chapter 10 introduces a device for forming liquid crystal films in-vacuo to a desired thickness at high repetition rates. This Linear Slide Target Inserter (LSTI) operates on the same basic film formation principles described in previous chapters but can operate at the $0.1 \mathrm{~Hz}$ target insertion scale. The design, capabilities, and operational techniques of the device will be presented.

Chapter 11 concludes this work with a brief outlook on the future of liquid crystal film targets. Due to the utility and promise of these films a number of experimental opportunities for the near future have emerged. This experimental time on several facilities around the 
world will allow a broad range of experiments to be realized, the core ideas of which will be introduced here. 


\section{Chapter 2 \\ THEORY OF ION ACCELERATION}

\subsection{Overview}

High Energy Density Physics (HEDP) grew as a field from the study of fusion interactions. It was recognized that the incredible pressures and temperatures necessary to achieve fusion could be accomplished with the forces and fields generated from highly amplified short pulses of light. As a field, effort has been focused toward larger lasers that can produce more extreme conditions, as well as faster firing lasers to facilitate more frequent experimental shots. The byproducts of producing such conditions in a controlled fashion are manifold: secondary radiation and particle beams are generated during the light interaction, all of which can be optimized by changing the nature of the laser, the target, or both. Applications are being pursued for all of these secondary radiation sources, to the point that many high powered laser systems in the world today have no interest in the original fusion energy application beyond the ubiquitous connection of studying similar fundamental processes.

One such application that encompasses much of the relevant physics and that interests a large portion of the community is ion acceleration. The details of these processes span many sub-fields of physics, from electromagnetism to plasma physics to relativistic hydrodynamics. These acceleration mechanisms depend critically on a number of laser and target parameters, chief among them being the thickness of the target, as will be described here. The nature of the ions accelerated, including their number, energy spectrum, spatial distribution, and species, is the subject of continued investigation in the field. This chapter begins with the relevant introductory physics for intense laser interaction with a plasma target, and then moves on to discuss the details of several mechanisms of ion acceleration. Included here is a description of the physical mechanism, the resulting spectral shape of accelerated ion energy, and an expression for the energy of the ions as a function of relevant laser parameters. 


\subsection{Initial laser interaction}

A typical simplification made early on in HEDP science was to reduce the physics of the laser pulse once it reached the target interaction region to a handful of numbersenergy, pulse duration, wavelength, and spot size. These values are enough to do the most fundamental calculations-chiefly, the intensity on target-but of course much of the relevant physics cannot be explained by so simple a construction. What follows here is a brief discussion of the most salient aspects a pulse has as it approaches and then begins interaction with a target, with some explanation of the origin of these effects.

\subsubsection{Laser pulse shape}

As a broad simplification, high powered laser systems can be grouped into two categories: those that achieve these powers via large amounts of energy (hundreds of joules up to tens of kilojoules on the largest systems) in moderately short pulses (tens of nanoseconds), and those that aim for much less energy (on the few or few tens of joules level) but pack this into a much shorter pulse (tens of femtoseconds at the shortest). While these regimes of pulse energy can have differing effects on a given experiment, the difference in pulse durations has more fundamental consequences. The femtosecond timescales of laser interaction for ultra-short pulse systems are sufficiently brief that only the electrons with their lower inertia are free to respond to the intense laser fields, with heavier ion motion typically following on picosecond timescales. The consequences of this fact alone have implications from particle acceleration to radiation production to the damage properties of optics.

Because the duration of the pulse is of such physical importance, knowledge of the temporal shape of the incoming pulse is critical for a complete understanding of the resulting physics. A discussion of the details of short pulse generation and amplification will follow in Chapter 4, but for now it will be sufficient to outline a few relevant points on the pulse journey to the target chamber.

Titanium-doped sapphire is the current gain medium of choice for ultra-short pulse laser systems due to its large gain bandwidth, relatively high damage threshold, and high saturation power, among other properties. The upper state lifetime of Ti:sapphire is 3.2 $\mu s$, relatively short compared to other media. Typical operation of a Ti:sapphire amplifier is to pump a cylindrical crystal with several watts of green spectral region pump power, for example the $532 \mathrm{~nm}$ light from a frequency-doubled Neodymium:Yttrium Aluminum Garnet (ND:YAG) system. The structure of Ti:sapphire is such that stimulated emission for wavelengths between $650 \mathrm{~nm}$ and $1100 \mathrm{~nm}$ is possible, with $800 \mathrm{~nm}$ being the common center for these systems.[14] The amplifier crystal will be pumped by the green light a few $n s$ before the short pulse light to be amplified enters the crystal.

It is typically advantageous to put enough pump energy into the crystal such that 
multiple passes are required to optimally extract it into the short pulse beam, but still there will be leftover energy that is not stimulated into emitting when the short pulse light is present. This energy will later emit as spontaneous emission, some of which will be collinear with the main beam path through this and any subsequent amplifiers. This light is called Amplified Spontaneous Emission (ASE), and typically exists as a pedestal of light surrounding the main pulse in time, usually many orders of magnitude reduced in energy. Optical shutters exist in the laser beam path that serve to limit this pedestal down to the tens of $n s$ level, and in a system with proper amplification timing will only amplify the ASE nearest the main pulse. The result is still some amount of somewhat amplified light extending at least nanoseconds in front of the main pulse, with the energy level of that pre-pulse light increasing by orders of magnitude once it is within a picosecond of the main pulse.

Additionally, strong peaks can be present on the nanosecond or picosecond time scales if an unwanted reflection can remain collinear with the main beam path. Though antireflection coatings exist to mitigate these issues, prepulses can still be amplified by many orders of magnitude as they travel along with the main pulse through the amplification process. Overall the difference in magnitude of the intensity of the main pulse and any surrounding prepulses or pedestals is referred to as the laser contrast. An especially high contrast laser may have 10 orders of magnitude between the main peak and any surrounding pulses, although even this level is not sufficient for some experiments, as will be discussed later.

It is worth noting that even though the pre-pulse light can be many orders of magnitude lower in intensity than the main pulse, it may still be easily strong enough to generate a plasma on the target on the scale of nanoseconds or picoseconds before the main pulse arrives. In this way the most intense portion of the laser pulse rarely interacts with a sharp vacuum/target interface, but rather impinges upon a preplasma generated by these leading pulse elements. Such a preplasma will expand in the time before the main pulse arrives, and is typically described by an exponential profile of the form

$$
n_{e} \propto \exp (-z / L)
$$

where $n_{e}$ is the electron density and $L$ is the characteristic plasma scale length, typically given in $\mu \mathrm{m}$. This preplasma on the front side of the target is where the incoming intense laser pulse will first interact with the target.

\subsubsection{Laser-plasma interactions}

Broadly, the physics that describes what occurs when the intense laser pulse reaches a plasma target is collectively called the Laser Plasma Interaction (LPI). This constitutes a large area of theory, modeling, and experimental study, and is in essence a field unto itself. 
This is an introduction to just the most basic effects and those that will be most useful for the subsequent discussion of ion acceleration.

Like any plasma, the preplasma on the front of the target is an overall charge neutral medium consisting of partially or fully ionized atoms. The plasma constituents will respond to disturbances in the charge distribution, and the electric field of the incoming laser beam is just such a disturbance. For example, electrons will shift in response to a charge imbalance, but will overshoot and then be pulled back by the subsequent imbalance, setting up an oscillation. The frequency of electrons oscillating in this manner is the plasma frequency $\omega_{p}$, given by

$$
\omega_{p}=\sqrt{\frac{n_{e} e^{2}}{\gamma_{L} m_{e} \epsilon_{0}}}
$$

where $e$ is the fundamental electron charge, $\gamma_{L}$ is the cycle-averaged Lorentz factor of the oscillating electrons, $m_{e}$ is the electron mass, and $\epsilon_{0}$ is the permittivity of free space. The Lorentz factor is directly related to the electric field of the laser, but is often more convenient to express in terms of the laser intensity. This can be done using the normalized laser vector potential, $a$ :

$$
a=\frac{e E}{m_{e} \omega_{L} c}=\sqrt{\frac{I \lambda^{2}}{1.37 \times 10^{18 \frac{W m^{2}}{c m^{2}}}} .}
$$

Here $\omega_{L}$ is the laser frequency. The parameter $a$ can be thought of as a ratio of an electron's gained momentum to its rest energy divided by the speed of light $c$, and as such relativistic effects become significant when $a$ approaches and exceeds $1 .{ }^{1}$

This plasma frequency determines the way that light propagates through a plasma, which can be expressed through the plasma's index of refraction

$$
n=\sqrt{\frac{\epsilon(\omega)}{\epsilon_{0}}} \simeq \sqrt{1-\frac{\omega_{p}^{2}}{\omega^{2}}} .
$$

With this expression and Maxwell's equations a dispersion relation for light traveling through a plasma can be obtained:

$$
\omega^{2}=\omega_{p}^{2}+c^{2} k^{2}
$$

where $k$ is the wave number. It is the plasma frequency, then, that determines how light travels through the plasma. As a laser moves through the preplasma toward the solid

${ }^{1} a$ is not strictly the ratio of these energies, and so can exceed 1 without violating any physical laws. It is often reduced to the expression $a \sim \frac{v_{o s c}}{c}$ where $v_{o s c}$ is the so-called non-relativistic "quiver" velocity induced by the oscillating electric field. For this expression it is also permissible to have $a>1$ despite appearances. Indeed, lasers with intensities of $10^{21} \mathrm{~W} / \mathrm{cm}^{2}$ have $a>10$. 
density target beyond, it will see an increasingly high electron density, and therefore an increasingly large plasma frequency.

Special consideration must be given to the plasma density where the local plasma frequency is equal to the incoming laser frequency-at this point the dispersion relation is only solved for $k=0$, which is equivalent to an infinite phase velocity and zero group velocity. Physically, the incoming laser field decreases exponentially past this surface of critical density because the electrons here are able to shift (at a speed determined by the local plasma frequency) and cancel out the incoming laser electric field, causing most of it to be reflected. This critical electron density is given by

$$
n_{c}=\frac{\gamma_{L} m_{e} \epsilon_{0} \omega^{2}}{e^{2}}
$$

For practical numbers, this critical density is given by $\sim 1.1 \times 10^{21}\left(\frac{\lambda}{\mu m}\right) \mathrm{cm}^{-3}$. As an example, a singly ionized copper plasma has electron density $n_{e} \approx 9 \times 10^{22} \mathrm{~cm}^{-3}$; this means a $\lambda=800 \mathrm{~nm}$ laser will not propagate through the solid density copper but will go through some amount of preplasma before being reflected at the critical density surface. The region of plasma before this critical density surface is called "underdense", in contrast to the "overdense" region beyond the critical surface where the plasma is too dense for light to propagate. The fact that light will not pass this point has important consequences for particle interaction.

An electron in the preplasma will respond to the oscillating fields of the incoming laser according to the Lorentz force law:

$$
\mathbf{F}=-e(\mathbf{E}+\mathbf{v} \times \mathbf{B}) .
$$

The first term dominates for low intensity interactions, and the electron will just oscillate in the direction of the laser polarization. For intensities of $I \sim 10^{18} \mathrm{~W} / \mathrm{cm}^{2}$ and above, the magnetic component of the Lorentz force becomes appreciable and the electron will gain a momentum component in the direction of laser propagation. Due to the oscillating nature of the electromagnetic fields, an electron will normally move forward longitudinally as it oscillates transversely, sweeping out a figure- 8 pattern in an average motion frame. However, if the forward longitudinal motion should move a preplasma electron past the critical surface it will continue on with the energy it just gained from the laser field, since past this point in the plasma there is no laser field to oscillate back and pull against the electron.

In this way electrons can permanently gain energy from the laser. For the ultra-high intense fields typical to short pulse lasers like Scarlet, this electron acceleration mechanism is sufficient to produce relativistic electrons within one optical cycle $(2.7 f s)$. The effect of the laser on this preplasma has been the subject of numerous studies as efforts to understand 
more about the fundamentals of HEDP.[15, 16] It is this cloud of accelerated (or "hot") electrons that forms the beginning of a number of ion acceleration mechanisms. Because of their important role as initiators for these mechanisms, numerous experiments[17, 18] and simulations $[19,20]$ have been performed to ascertain the details of hot electron generation and propagation through the plasma target.

A final point critical to ion acceleration via the mechanisms to be discussed lies with the ponderomotive force produced by the spatially varying laser. This effect arises from the interaction of an electron with the inhomogeneous oscillating electromagnetic field of the incoming laser. Physically, the ponderomotive force imparts energy to the laser in the following way: an electron oscillating in a field with a spatial gradient will experience different magnitudes of Lorentz force as it oscillates through this varying electric field. The result is a weaker push from regions of weaker electric field, which can be described as an effective force on the center of mass motion of the charged particle. In this way the ponderomotive force pushes electrons away from regions of stronger fields-i.e. the laser pushes electrons away from its Gaussian focus. The ponderomotive force $F_{p}$ has the form[10]

$$
\mathbf{F}_{\mathbf{p}}=-m_{e} c^{2} \nabla\left(1+\langle\mathbf{a}\rangle^{2}\right)^{1 / 2}=-\frac{e^{2}}{4 m_{e} \omega^{2}} \nabla|E|^{2}(1-\cos 2 \omega t)
$$

Here the rightmost expression assumes a linearly polarized plane wave. The forcing from this expression is critical to one of the acceleration mechanisms to be discussed and will be revisited later.

\subsection{TNSA}

\subsubsection{Physics of TNSA}

Since the first experiments around 2000,[21, 22] the Target Normal Sheath-field Acceleration (TNSA) mechanism has been studied as the chief method of ion acceleration using intense lasers. Briefly, TNSA begins when a sufficiently intense part of the laser pulse interacts with any pre-plasma that has formed on the front surface of the target. The large electric field from the incoming intense laser pulse will accelerate electrons within this pre-plasma to relativistic energies, as described in the previous section. The fast electrons shoot through the target and exit the rear side, leaving behind a net positively charged bulk target. Crucially, this process is occurring on femtosecond timescales, so the inertia of the much heavier ions prevents them from moving initially in response to this electron acceleration. The resulting charge imbalance creates an electric field on the back of the target that points normal to the surface and towards the advancing electron cloud. A cartoon of this process is shown in Fig. 2.1. Typically on the order of $M V / \mu m$ for ultra-short lasers, this TNSA field will accelerate ions on the rear surface of the target up to tens of $\mathrm{MeV} /$ nucleon energies. 


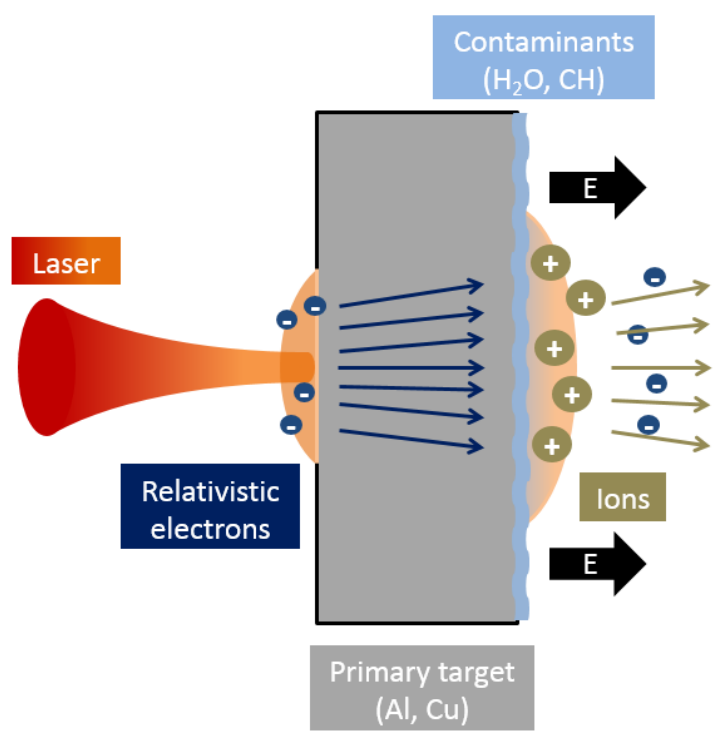

Figure 2.1: Cartoon showing the critical elements of the TNSA process. Here relativistic electrons accelerated from the front target surface by an incident laser leave the rear of the target, setting up a strong electric field between this accelerated electron cloud and the remaining positively charged target. This field accelerates charged particles from the back of the target, usually including contaminants inherent to $10^{-6} \mathrm{Torr}$ vacuum chambers.

The details of this interaction as well as the relevant physical models will be presented below with an emphasis on how this process relates to other ion acceleration mechanisms.

Since it is these hot electrons that generate the TNSA field, their energy distribution has a controlling effect on the ultimate accelerated ion energy distribution. At early laser interaction times the fastest electrons have left the target and set up the initial sheath field for ion acceleration. Electrons accelerated at later times will experience this sheath field as well, being pulled back toward the target. Those without sufficient energy to escape will return to the target, reducing the charge differential and therefore the sheath field magnitude. In this way ions accelerated at late times will have lower energies. The resulting accelerated ion spectrum is broad with an exponential shape, resembling the electron distribution that produced them.[17, 19]

Critically, this acceleration always occurs along the rear target surface normal direction, since the driving field is set up between this positively charged rear surface and the accelerated hot electron cloud beyond. This was shown definitively by Snavely et al.,[22] where $100 \mu \mathrm{m} \mathrm{CH}$ targets with a wedge morphology were shot with a $3 \times 10^{20} \mathrm{~W} / \mathrm{cm}^{2}, 1$ $\mu m$ wavelength, few-ps pulse duration laser. In the plane of laser reflection these targets had triangular cross section such that the incoming laser was incident on one long face. 
The resulting accelerated ions were recorded on radiochromic film $(\mathrm{RCF})^{2}$, where a 300 $\mu m$ tantalum filter blocked protons below $18 \mathrm{MeV}$ energies. Though there is some inherent angular spread in the TNSA mechanism, the RCF showed much greater proton yield leaving the two rear normal directions of this target, proportioned according to the location of the incoming laser $k$-vector with respect to each rear target face.

It is useful to remember a few scaling laws so that quick calculations can be done in response to experimental ideas or initial analysis. The TNSA field is set up initially by fast electrons accelerated by the laser, which will inherently have some energy spread due to the nature of their initial laser interaction. As the sheath field grows some lower energy electrons will not have sufficient speed to escape and will be pulled back toward the target. This in turn decreases the space charge separation and so too the sheath field magnitude, so that typically ions accelerated later will leave the target with lower energies over time. This back-holding of electrons by the sheath field will in general depend on the hot electron temperature $T_{e}$ (or, the energy spread in accelerated electrons) and the length scale of the plasma at the back of the target $L_{s}$, so a reasonable equation is

$$
e E_{S} \sim \frac{T_{e}}{L_{s}} \sim \frac{T_{e}}{\lambda_{D}}
$$

where $E_{S}$ is the TNSA sheath field strength and $\lambda_{D}=\sqrt{\frac{\epsilon_{0} k_{B} T_{e}}{e^{2} n_{e 0}}}$ is the electron Debye length. For typical numbers like intensity $I \sim 10^{20} \frac{\mathrm{W}}{\mathrm{cm}^{2}}, 10 \%$ conversion efficiency of laser energy into electron energy, hot electron temperature $T_{e} \sim 5.1 m_{e} c^{2}=2.6 \mathrm{MeV}$, hot electron density $n_{e} \sim 8 \times 10^{20} \mathrm{~cm}^{-3}$, and therefore Debye length $\lambda_{D}=4.2 \times 10^{-5} \mathrm{~cm}$, the sheath field strength will be $E_{S} \sim 0.6 \frac{M V}{\mu m}$, which is sufficient to accelerate protons on the rear target surface to tens of $\mathrm{MeV}$ energies.

\subsubsection{Theory of TNSA}

The following discussion follows closely that by Mora[23] and Macchi et al.[24]

\section{Quasi-static model}

Theoretical models of TNSA fall into two categories. First are those that assume light ions get accelerated early in the sheath formation, so that the sheath is assumed to be stationary; these are quasi-static models. Second, dynamic models begin with a neutral plasma where ions gain kinetic energy during sheath evolution.

Under the quasi-static approximation, a background of immobile, heavy ions is assumed, and that there are sufficiently few light ions to neglect their effect on particle evolution. Additionally, the laser-produced hot electron population is considered isothermal.

\footnotetext{
${ }^{2} \mathrm{RCF}$ is an important tool for accelerated ion detection, and will be discussed in greater detail in Chapter
} 9 where it is used in one of the presented experiments. 


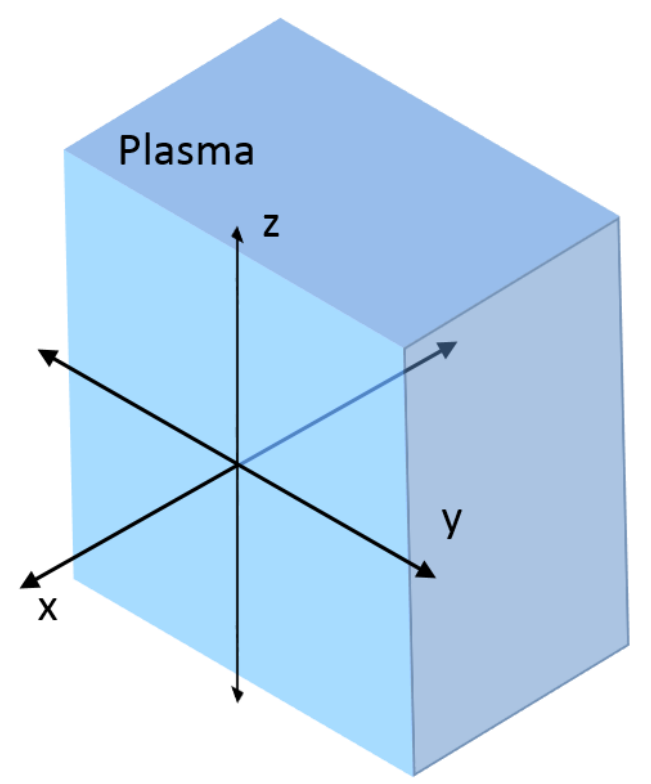

Figure 2.2: Schematic for quasi-static theoretical model, wherein a plasma of electrons and immobile heavy ions occupies the half space $x<0$.

In the following the electrons within the plasma have a continuous Boltzmann distribution described by $n_{e}=n_{e 0} \exp \left(\frac{e \Phi}{k_{B} T_{e}}\right)$, where $\Phi$ is the electrostatic potential, $k_{B}$ is the Boltzmann constant, $T_{e}$ is the hot electron temperature, and the unperturbed electron density is given by $n_{e 0}=Z n_{i 0}$ where $Z$ is the electron number per atom and $n_{i 0}$ is the ion number density. A final assumption lies in the choice of electrostatic potential boundary condition: $\Phi(-\infty)=0$.

The relevant expression to solve is the 1D planar Poisson equation:

$$
\epsilon_{0} \frac{\partial^{2} \Phi}{\partial t^{2}}=e\left(n_{e}-Z n_{i}\right)
$$

The solution for the region $x>0$ is of the form

$$
\Phi(x)=\frac{-2 k_{B} T_{e}}{e}\left[\ln \left(1+\frac{x}{\sqrt{2 e_{N}} \lambda_{D}}\right)+1\right]
$$

where $e_{N}$ is Euler's number, to distinguish it from the electron charge $e$, and $\lambda_{D}$ is the Debye length for the unperturbed plasma electron density.

The electric field at the plasma surface resulting from this potential is given by

$$
E(0)=-\nabla \Phi(0)=\frac{2 k_{B} T_{e}}{e} \frac{1}{\sqrt{2 e_{N}} \lambda_{D}}=\sqrt{\frac{2}{e_{N}}} E_{0}
$$




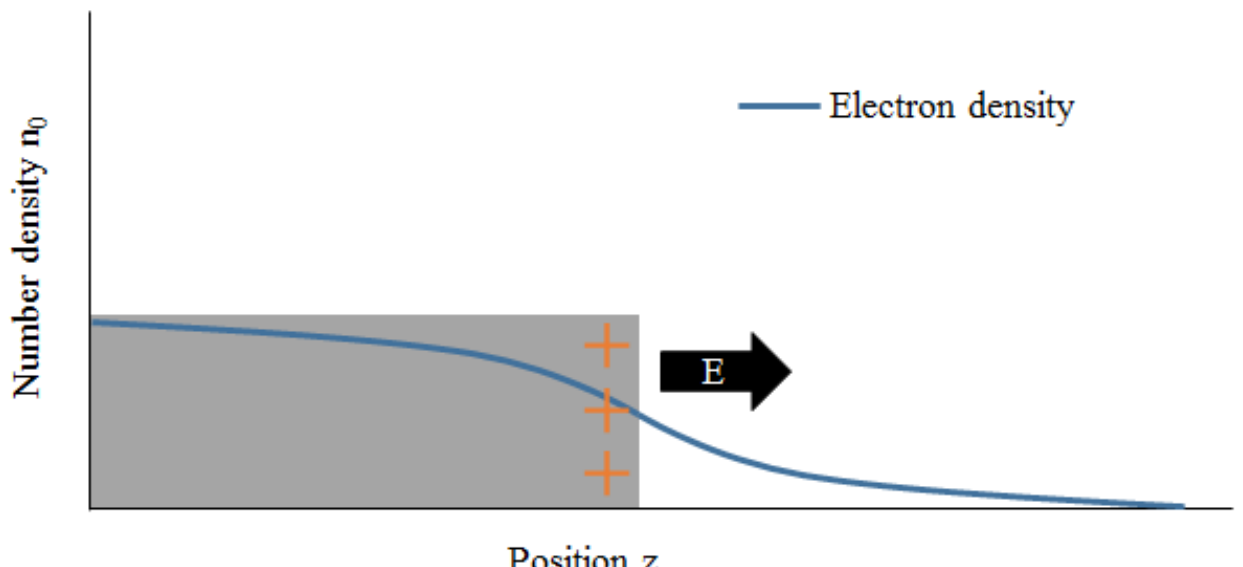

Figure 2.3: Cartoon of the immobile heavy ion population (grey) and Boltzmann electron distribution (blue). The resulting charge separation creates an electric field directed away from the target.

for

$$
E_{0}=\frac{k_{B} T_{e}}{e \lambda_{D}}
$$

As mentioned previously, the accelerating sheath field scales linearly with hot electron temperature $T_{e}$ and inversely with Debye length. A cartoon of the initial distributions and the resulting sheath field is shown in Fig. 2.3.

The problem with Eq. 2.11 is that the magnitude of the potential increases to infinity as $x$ increases, implying infinite acceleration far away from the target. This is unphysical, and so a few constraining assumptions may be made. A cutoff energy $\epsilon_{c}$ can be assumed for large $x$ values such that $e \Phi \rightarrow-\epsilon_{c}$ as $x \rightarrow \infty$. Alternatively a phenomenological cutoff acceleration time can be mandated-a typically crude estimate for such a time is $1.3 \times \tau_{0}$ for pulse width $\tau_{0}$.

A further solution is to allow the ions to move as a fluid throughout the interaction, which constitutes the Dynamic model of the following section.

\section{Dynamic model}

The dynamic model begins with the same setup-ions occupying half space $x<0$-and describes the physics with fluid equations:

$$
\frac{\partial \mathbf{u}_{\mathbf{j}}}{\partial t}+\mathbf{u}_{\mathbf{j}} \cdot \nabla \mathbf{u}_{\mathbf{j}}=-\frac{Z e}{m_{j}} \nabla \Phi
$$




$$
\frac{\partial n_{j}}{\partial t}+\nabla \cdot\left(n_{j} \mathbf{u}_{\mathbf{j}}\right)=0
$$

Here $\mathbf{u}_{\mathbf{j}}$ describes the fluid velocity, and $m_{j}$ and $n_{j}$ refer to the mass and number density of electron or ion species $j$. These equations have a solution in a self-similar form ${ }^{3}$ :

$$
n_{i}=n_{0} \exp \left(-\frac{x}{c_{s} t}-1\right), \quad u_{i}=c_{s}+\frac{x}{t},
$$

where $c_{s}=\left(\frac{Z k_{B} T_{e}}{m_{i}}\right)^{1 / 2}$ is the ion sound speed. Using this solution in Eq. 2.14 yields the self-similar electric field $E_{s s}$ :

$$
E_{s s}=\frac{k_{B} T_{e}}{e c_{s} t}=\frac{E_{0}}{\omega_{p i} t}
$$

where $\omega_{p i}=\left(\frac{n_{e 0} Z e^{2}}{m_{i} \epsilon_{0}}\right)^{1 / 2}$ is the ion plasma frequency.

Inspection of this result will reveal an infinite solution for the electric field, this time at $t=0$. This can be accounted for by setting a physical cut-off length for interaction with the electric field. A reasonable procedure to find such a cut-off length is to set the local ion density scale length equal to the local Debye length:

$$
L \equiv \frac{n_{i}}{\left|\partial_{x} n_{i}\right|}=c_{s} t=\lambda_{D}=\lambda_{D 0}\left(\frac{n_{e 0}}{n_{e}}\right)^{1 / 2}
$$

Inserting Eq. 2.16 and solving for $x$ yields

$$
x(t)=c_{s} t\left[-1+2 \ln \left(\omega_{p i} t\right)\right]
$$

which gives the ion front position as a function of time. Taking a derivative of this expression yields the ion velocity as a function of time:

$$
u(t)=c_{s}\left[1+2 \ln \left(\omega_{p i} t\right)\right] .
$$

Finally, this ion front velocity leads to an acceleration that can be used with the Coulomb law to yield the electric field at the ion front location $E_{\text {front }}$ as

$$
E_{\text {front }}=2 \frac{k_{B} T_{e}}{e c_{s} t}=2 E_{s s}
$$

which is twice the self-similar field found in Eq. 2.17 earlier.

\footnotetext{
${ }^{3}$ Self-similar solutions are often seen in differential equations like these fluid relations, but as a mathematical construct can be somewhat poorly explained in the literature. Briefly, a self-similar solution is one that appears the same at all length and/or time scales. These solutions are always written in terms of a dimensionless self-similar variable, which is $\frac{x}{c_{s} t}$ here, so that the distribution will follow the form in Eq. 2.16 as this parameter is tuned over large and small values. Self-similar equations like this one are what allow some plasma codes to provide solutions for both the collision of intergalactic dust clouds as well as the interaction of micron scale length plasmas created by intense laser fields.
} 
As has been seen, both the quasi-static and dynamic models have infinities at different boundary conditions. Both of these can independently be handled with an appropriate choice of constraint, but perhaps a more satisfactory model can be produced from the interpolation of these two models.

\section{Interpolation}

The quasi-static solution found above fails at late times, where it predicts infinite energy, and the dynamic model fails at short times since it is singular at $t=0$. A suitable interpolation between these two models can be found in the form of

$$
E_{\text {front }} \approx \frac{2 E_{0}}{\left(2 e_{N}+\omega_{p i}^{2} t^{2}\right)^{1 / 2}}
$$

which reduces to the quasi-static result in the limit $t \rightarrow 0$ and the dynamic result in the limit $\omega_{p i} t \gg 1$. This interpolated solution for the electric field at the ion front can be used in Coulomb's law and integrated to return the ion front velocity and position:

$$
\begin{gathered}
u_{\text {front }} \approx 2 c_{s} \ln \left(\tau+\sqrt{\tau^{2}+1}\right) \\
x_{\text {front }} \approx 2 \sqrt{2} e_{N} \lambda_{D 0}\left[\tau \ln \left(\sqrt{\tau^{2}+1}+\tau\right)+\sqrt{\tau^{2}+1}+1\right]
\end{gathered}
$$

where $\tau=\frac{\omega_{p i} t}{\sqrt{2 e_{N}}}$, and where the constants of integration have been set with the initial conditions $u_{\text {front }}(0)=0$ and $x_{\text {front }}(0)=0$.

Finally, the velocity at late times is given by $u_{\text {front }}\left(\omega_{\text {pi }} t \gg 1\right) \approx 2 c_{s} \ln (2 \tau)$, which means the maximum energy $\mathcal{E}_{\max }$ in these ions is

$$
\mathcal{E}_{\text {max }} \approx \frac{1}{2} m_{i} u_{\text {front }}^{2}=2 Z k_{B} T_{e}[\ln (2 \tau)]^{2}
$$

\section{Quick TNSA math}

A good experimentalist will have the results of models such as these three presented here on hand, if not their explicit derivation, but more importantly needs to be able to perform quick calculations to determine that validity of raw data or discuss proposed experimental plans in real time. Here are some approximations and numbers to keep in mind pertaining to the physics of TNSA. This section follows the review by Daido et al.,[25] which provides numbers appropriate for a reasonable intensity contrast, $300 \mathrm{fs}$ to few-ps pulse width laser with focused intensity up to $10^{19} \mathrm{~W} / \mathrm{cm}^{2}$ interacting with a reasonably thick $(10 \mu \mathrm{m}$ or more) planar target. Details are provided as to the application of these approximations to shorter pulse, higher intensity lasers. 
First the electron temperature scaling with laser intensity is given by[19]

$$
T_{e}=m_{e} c^{2}\left[\sqrt{1+a_{0}^{2}}-1\right] \simeq a_{0} m_{e} c^{2} .
$$

Here $a_{0}$ is the peak normalized laser vector potential (and so is calculated at the peak electric field value). This scaling serves as a reasonably good approximation for the hot electron temperature generated by high intensity lasers, which as previously mentioned determine the details of the subsequent ion acceleration. Higher intensity, higher contrast lasers tend to follow the experimental Beg scaling, [17] given by:

$$
T_{e} \approx 0.47 m_{e} c^{2} a_{0}^{2 / 3} \approx 0.22 M e V\left(I_{18} \lambda_{\mu m}\right)^{1 / 3}
$$

where $I_{18}$ and $\lambda_{\mu m}$ are the intensity in units of $10^{18} \mathrm{~W} / \mathrm{cm}^{2}$ and the wavelength in terms of $\mu m$, respectively. These scaling laws, of $1 / 2$ or $1 / 3$ power, are typical of TNSA experiments and set some limits on the maximum proton energy attainable with a given laser system.

As mentioned previously, the acceleration time for ions at the rear target surface can be roughly approximated by a small multiple of the pulse width: $1.3 \tau_{0}$. This is less true for sub-100 fs pulses, where the acceleration time extends somewhat longer than the duration of the pulse due to the additional heating from refluxing hot electrons (those that travel through the target multiple times due to not having enough energy to escape the TNSA sheath field).

A straightforward estimation of the sheath radius $r_{s}$ can be obtained from knowing the laser spot radius $r_{0}$, the target length $l$, and electron divergence angle $\theta$ :

$$
r_{s}=r_{0}+l \tan \theta
$$

As a rough assumption this sheath radius is accurate out to around $2 r_{s}$ beyond the rear surface of the target.[25]

The conversion efficiency of laser energy into hot electrons can be obtained from

$$
\eta=1.2 \times 10^{-15}\left(\frac{I_{0}}{W / \mathrm{cm}^{2}}\right)^{0.74}
$$

where $I_{0}$ is the maximum laser intensity. This equation is only valid for intensities up to around $10^{19} \mathrm{~W} / \mathrm{cm}^{2}$, at which point the electron conversion efficiency plateaus around $40 \%$, although this number can vary significantly with laser energy, pulse duration, and contrast level.[26]

Finally, the number $N_{e}$ and density of hot electrons within the sheath, assuming a cylindrical electron cloud with length and radius equal to the laser pulse length and sheath 
radius, respectively, and a laser energy $E_{L}$, are given by

$$
N_{e}=\frac{\eta E_{L}}{T_{e}} \quad n_{e 0}=\frac{N_{e}}{c \tau_{0} \pi r_{s}^{2}} .
$$

As a last approximation, Macchi et al. [24] have established an empirical formula for the maximum electron energy obtainable from TNSA as a function of the input laser energy in $J$ :

$$
\mathcal{E}_{e, \max }=4.8+0.8 \ln \left(E_{L}\right) .
$$

This expression fits collected data reasonably well for pulse widths between $40-900 \mathrm{fs}$ and laser energies from $0.1-500 J$.

Experimentally, the maximum ion energy from TNSA has been found to scale with the cube root of the intensity.[17] At the time of this writing the energies required for a number of applications, including proton cancer therapy, cannot be reached with ions accelerated via the TNSA mechanism. This is partially due to these scaling laws, but many think there are other processes at work that are poorly understood at this stage. Research continues into optimizing and understanding the TNSA mechanism, some of which has revealed other ion acceleration processes that may be more useful for achieving higher energies.

\subsection{Radiation Pressure Acceleration}

\subsubsection{Comparison to TNSA}

TNSA is certainly the most explored and most easily attained method of ion acceleration. However, continuing upgrades in laser contrast have allowed experiments with thinner and thinner targets, revealing a new acceleration mechanism. In Radiation Pressure Acceleration (RPA), the momentum of the electromagnetic wave is transferred directly to the target via the ponderomotive force, and in sufficiently thin targets this allows the entire irradiated area to be accelerated along with the passing laser field. The resulting ion acceleration has a few critical differences when compared to TNSA: namely that the acceleration direction is along the laser axis rather than the target normal direction, and also that the resulting ion energy spectrum often has a somewhat monoenergetic component instead of the typical exponential spectrum of TNSA. The theoretical and experimental details of this RPA interaction will be outlined here with consideration to their comparison to TNSA.

The idea for utilizing the radiation pressure of a large amount of light stems from a paper by Maxwell in 1860, and was suggested as a method for space travel in stories by Jules Verne. The utilization of solar fluxes of light was the most promising avenue for this until the first laser fired in 1960, after which a number of theoretical papers emerged discussing their use for radiation pressure. While models on this effect and its role in ion 
acceleration and related high intensity laser applications have been published for a few decades now, [24, 27-29] there have only been a few experiments that can claim to have measured an ion spectrum arising entirely or even chiefly from this effect.

\subsubsection{Theory of RPA}

A simple derivation for the expression for the radiation pressure of light comes from relating the intensity of a plane, monochromatic light wave with the momentum it carries and therefore the pressure it exerts. ${ }^{4}$ For light incident on a medium that will Fresnel reflect with some reflection, transmission, and absorption coefficients $R, T$, and $A$, respectively, the expression for the radiation pressure is given by

$$
P_{\text {rad }}=(1+R-T) \frac{I}{c}=(2 R+A) \frac{I}{c} .
$$

This pressure is mediated through the ponderomotive force defined earlier:

$$
\mathbf{F}_{\mathbf{p}}=-\frac{e^{2}}{4 m_{e} \omega^{2}} \nabla|E|^{2}(1-\cos 2 \omega t)
$$

Of note here is the difference in ponderomotive force for linear or circular polarizations: for circularly polarized light the ponderomotive force is constant because the $\cos (2 \omega t)$ term does not appear. For this reason, circular polarization was first modeled and used in experiment to perform RPA, since setting up a stable balance between the ponderomotive and electrostatic pressures to generate a clean RPA effect is necessary to observe this mechanism. Results from linearly polarized pulses eventually followed as better target materials and thicknesses were used.

An incident laser will move through any underdense preplasma created on the front side of the target before reaching the critical density, where it reflects. The ponderomotive force on this region will tend to shape the critical surface somewhat[30] and move it forward. This oscillation and reflection can be described by the moving mirror model, which is necessary here to find the radiation pressure in the instantaneous reference frame of the mirror. The frequency of incoming light $\omega$ will shift at this mirror surface according to the relativistic Doppler equation: $\omega^{\prime}=\sqrt{\frac{1-v / c}{1+v / c}}$, which ultimately results in a Doppler shift of the incident intensity $I_{0}{ }^{5}$ :

$$
I^{\prime}=I_{0}\left(\frac{1-\beta}{1+\beta}\right) \rightarrow P_{r a d}=\frac{2 I}{c}\left(\frac{1-\beta}{1+\beta}\right)
$$

${ }^{4}$ The radiation pressure $P_{\text {rad }}$ can be related back to intensity through the force $F$, area $A$, momentum $p$, energy $U$, and Poynting vector $\langle S\rangle$ as follows: $P_{\text {rad }}=\frac{\langle F\rangle}{A}=\frac{1}{A}\left\langle\frac{d p}{d t}\right\rangle=\frac{2}{A c}\left\langle\frac{d U}{d t}\right\rangle$, and $\left\langle\frac{d U}{d t}\right\rangle=\langle S\rangle A=I A$, so ultimately $P_{\text {rad }}=\frac{2 I}{c}$.

${ }^{5}$ Though not a common mode of thinking for high intensity laser scientists, the intensity can be thought of as $N$ photons each with energy $\hbar \omega$ impacting the target surface within a short time $\tau$, in which case a Doppler shift in the incident frequency results in the same shift in the incident intensity. 
Here $\beta=\frac{v}{c}$. An estimation of the velocity of the receding mirror surface, or hole boring velocity $v_{h b}$, may be found by balancing the incoming radiation pressure with the resulting momentum transfer. Here ions with density $\gamma_{h b} n_{i}$ for $\gamma_{h b}=\left(1-v_{h b} / c\right)^{-1 / 2}$ are incident with velocity $v_{h b}$ on the stationary mirror (in the mirror reference frame), and upon impact and reflection transfer momentum equal to $2 \gamma_{h b} m_{i} v_{h b}$, resulting in the balance

$$
\frac{2 I}{c} \frac{1-v_{h b} / c}{1+v_{h b} / c}=n_{i} \gamma_{h b}\left(2 \gamma_{h b} m_{i} v_{h b}\right) v_{h b}
$$

which, upon solving for the hole boring velocity, yields

$$
\frac{v_{h b}}{c}=\frac{\Pi^{1 / 2}}{1+\Pi^{1 / 2}}, \quad \Pi=\frac{I}{m_{i} n_{i} c^{3}}=\frac{Z n_{c} m_{e}}{A n_{e} m_{i}} a_{0}^{2} .
$$

This results in a maximum acceleration ion energy of

$$
\mathcal{E}_{\text {max }} \approx 2 m_{i} c^{2} \frac{\Pi}{1+2 \Pi^{1 / 2}}
$$

In the non-relativistic limit $v_{h b} \ll c$ and $\Pi \ll 1$, these two equations reduce to $\frac{v_{h b}}{c} \simeq \Pi^{1 / 2}$ and $\mathcal{E}_{\text {max }} \simeq 2 m_{i} c^{2} \Pi$.

This process proceeds as follows: the forward-directed ponderomotive force pushes electrons within the plasma forward by a skin depth, and the resulting electrostatic field between these electrons and the stationary heavy ions accelerates some of those ions. Eventually the ions will be pulled forward and accelerated by an electrostatic field produced between the two charged regions. At this point the ions and electrons can be pushed forward by the laser as a quasi-neutral plasma slab. For thick targets, subsequent laser penetration can cause the electron bunching process to start again, accelerating more ions. This acceleration and subsequent penetration of the laser gives this process the name "holeboring". Though this process is described as pulsed, it is in fact a continuous acceleration because the electron cloud with its lower inertia will respond fluidly to the motions of the moving ions.[31] A cartoon of this pulsed process involving laser pushing of electron density and then acceleration due to the resulting field is shown in Fig. 2.4.

Critically, this acceleration occurs at the front side of the target and sends ions along the direction of the incoming laser with velocity $2 v_{h b}$. Since the most electrons are accelerated at a maximum of this ponderomotive pressure-induced field, there will be a somewhat monoenergetic spike in the resulting ion spectrum behind the target.[32] The electrons accelerated during this process that leave the rear side of the target will also contribute to TNSA acceleration, and so targets shot at or close to normal incidence often have some accelerated ion spectrum contribution from both of these physical mechanisms. Even so, the difference in the energy spectrum of accelerated ions (exponential from TNSA and quasi-monoenergetic from RPA) constitutes one possible method for distinguishing them 


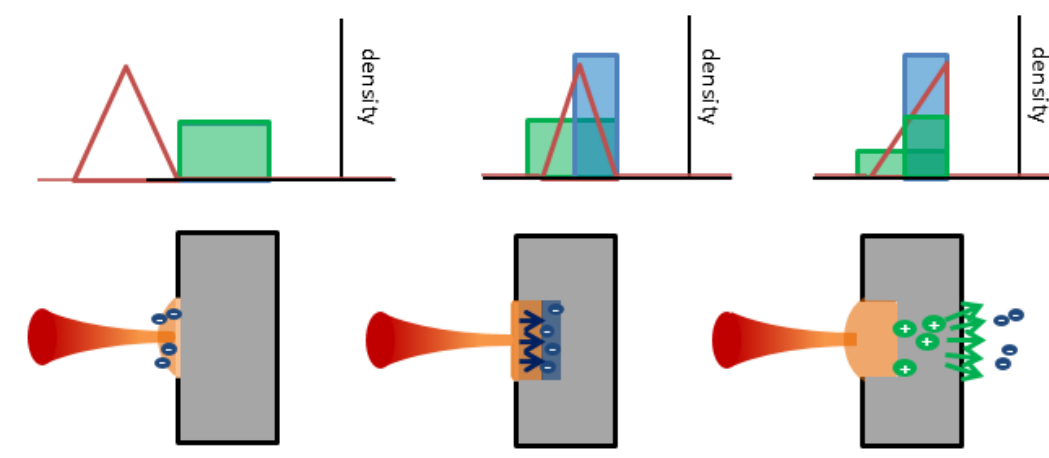

Figure 2.4: Cartoon depicting RPA. Each column is one step of the process, proceeding in time from left to right. The top row depicts laser intensity and electron and ion distribution profiles, while the bottom shows the location of those elements for each step. Initially the laser pushes the electron density forward before the ions have a chance to move (second column), creating an electric field in between these populations that will subsequently accelerate ions. At later times the ions are free to move as well, and for sufficiently long laser pulses there can be continued stages of electron density increase and ion acceleration.

when a target is shot near normal incidence.

For targets with thickness near the skin depth of their material, the laser may be able to accelerate all ions within the target before the pulse has completely reflected, resulting in penetration of some or all of the pulse and continued acceleration of target ions by the forward propagating laser. This thin target RPA is often called the light sail regime for its similarity to a proposed method of space travel using solar radiation pressure on a large sail.

Expressions for this light sail regime of RPA can be obtained beginning with Newton's 2nd law again:

$$
\frac{d p}{d t}=\frac{\gamma m_{i} c \beta}{d t}=\frac{2 I\left(t_{r e t}\right)}{c} R\left(\omega^{\prime}\right) \frac{1-\beta}{1+\beta}
$$

where this equation uses the retarded time $t_{r e t}=t-X / c$ for mirror position $X$ and the reflectivity as a function of Doppler shifted frequency $R\left(\omega^{\prime}\right)$ because the sail is moving. Rearranging and combining variables results in

$$
\frac{d(\beta \gamma)}{d t}=\frac{2 I\left(t_{r e t}\right)}{\sigma c^{2}} R\left(\omega^{\prime}\right) \frac{1-\beta}{1+\beta}, \quad \frac{d x}{d t}=\beta c
$$

where $\sigma=m_{i} n_{i} l$ is the mass density per unit surface. This equation can be solved for 
an arbitrary intensity to yield the following:[33]

$$
\beta\left(t_{r e t}\right)=\frac{\left[1+\mathcal{F}\left(t_{r e t}\right)\right]^{2}-1}{\left[1+\mathcal{F}\left(t_{r e t}\right)\right]^{2}+1}, \quad \mathcal{F}\left(t_{r e t}\right)=\frac{2 F\left(t_{r e t}\right)}{\rho l c^{2}}
$$

where $\rho$ is the mass density per volume of the target and $F\left(t_{r e t}\right)=\int_{0}^{t_{r e t}} I\left(t^{\prime}\right) d t^{\prime}$ is the laser fluence on target. The corresponding maximum ion energy from this light sail RPA model is

$$
\mathcal{E}_{\text {max }}=m_{i} c^{2}\left[\gamma\left(t_{r e t}\right)-1\right]=m_{i} c^{2} \frac{\mathcal{F}^{2}\left(t_{r e t}\right)}{2\left[\mathcal{F}\left(t_{r e t}\right)+1\right]} .
$$

Finally, the efficiency of laser energy to foil energy is given by

$$
\eta=\frac{2 \beta\left(t_{r e t}\right)}{1+\beta\left(t_{r e t}\right)}=1-\frac{1}{\left[\mathcal{F}\left(t_{r e t}\right)+1\right]^{2}} .
$$

To obtain the maximum foil velocity and accelerated ion energy, the dimensionless fluence parameter $\mathcal{F}$ can be evaluated in the limit at infinite time, where it has the value

$$
\mathcal{F} \simeq 2.2 \frac{F}{10^{8} \mathrm{~J} / \mathrm{cm}^{2}} \frac{1 \mathrm{~g} / \mathrm{cm}^{3}}{\rho} \frac{10 \mathrm{~nm}}{l}
$$

For Scarlet units of fluence $F \approx 10^{8} \mathrm{~J} / \mathrm{cm}^{2}$ and the $\rho=1 \mathrm{~g} / \mathrm{cm}^{3}$ of liquid crystal targets, this gives a dimensionless fluence of 0.22 for a $100 \mathrm{~nm}$ film. Using this in Eq. 2.41 gives a maximum accelerated ion energy of $\sim 30 \mathrm{MeV}$. In general, this model suggests that the optimum target thickness is around $\left.1.5 \times a_{0}[\mathrm{~nm}]\right)$. [33] Importantly, this model suggests a linear scaling of ion energy with fluence and therefore laser intensity, in contrast with the square or cube root scaling with the TNSA process.

Qiao et al.[32] derived an expression for the dominant regime of RPA over TNSA by constraining the target thickness to be sufficiently thin such that the hole boring effect can propagate to the rear target surface before the pulse is over, or $l_{0}<v_{h b} \tau$. Additionally, ions accelerated from the front of the target need to be faster than those accelerated later at the rear, such that the two groups merge to be accelerated together by the puncturing laser. Using these constraints along with the above expression for hole boring velocity and TNSA rear ion velocity, Qiao et al. obtained the following conditions:

$$
\frac{1}{\pi} \frac{n_{c}}{n_{0}} a<\frac{l_{0}}{\lambda}<\frac{1}{2 \pi} \sqrt{\frac{n_{c}}{\gamma n_{0}}} a \exp \left[\sqrt{\frac{Z n_{c}}{n_{0}}} \frac{a}{\sqrt{\gamma-1}}\left(\frac{n_{0}}{\gamma n_{c}}\right)^{1 / 4}+\frac{1-\ln 2}{2}\right] .
$$

Most of the difficulty with studying RPA lies with the past generation of laser technology: intensity contrasts on these lasers were typically in the $10^{-6}$ range, which is sufficient for thick $(>$ few- $\mu m$ ) target interaction. Thinner targets on the scale of $1 \mu \mathrm{m}$ and below will be given large underdense preplasma regions or fully obliterated by low contrast lasers before the main pulse arrives, rendering efficient ion acceleration from these 
target thicknesses impossible. Modern lasers are being built with contrast improvements in the form of Cross-Polarized Wave (XPW) system[34] (to be detailed in Chapter 4), parametric amplifiers as front ends, [35] or with upgrades that increase the contrast such as Pockels cell optimization, replacement of transmissive optics to reflective ones, [36] or with the implementation of plasma mirrors post pulse compression.[37]

Despite the early stages of experiment on RPA, simulations have shown that it should scale more favorably with laser intensity than TNSA.[24] Additionally, these numbers take into account only the RPA process, but it is thought that these two and other ion acceleration mechanisms may work together. The details of the onset and optimization of these and other ion acceleration mechanisms have not been fully determined, and is the aim of some work to be presented here.

Still other ion acceleration mechanisms have been recently identified, including the Breakout Afterburner (BOA) mechanism.[38, 39] This mechanism is thought to occur for extremely thin targets, in the sub-100 to several-hundred $\mathrm{nm}$ range, and as a result can only be observed on laser systems with excellent contrast. As such it is thought to have been observe in only a few experiments. The key physical difference between this and RPA is the onset of relativistically induced transparency (RIT), where an intense laser pulse can pass through overdense plasma regions because of a relativistic modification to the local plasma frequency. This results in an acceleration of the entire volume of electrons and ions within the target. Some more detail of the RIT process will be discussed in Chapter 11. It is worth mentioning briefly here, however, as another ion acceleration mechanism that depends critically on target thickness. 


\section{Chapter 3 \\ History of High Repetition Rate ION ACCELERATION SCHEMES}

\subsection{Overview}

The major impact of this work on liquid crystal targets hinges on their use as high repetition rate targets. As such some time should be spent on why high repetition rate previously has not been a priority for the high intensity laser community, what applications it now unlocks, and what target systems have been developed prior to this work.

Figure 3.1 shows the repetition rate in $H z$ of high intensity laser systems over time. In general this graph shows just the highest intensity facility at the time, which encompasses a number of laser system architectures. For the past few decades these large-scale lasers have been rate limited chiefly by the cool down time of the amplifier systems. Typically large glass disks or rods, these amplifier gain media would be pumped with eventually tens of joules of often $532 \mathrm{~nm}$, ns duration light, just before the seed beam was sent through to absorb the energy stored within the glass electronic structure. Part of a four-level laser system are two non-radiative transitions where electrons lose energy by exciting phonons in the material structure which eventually manifest as increased heat. The magnitude of this temperature increase on some systems was enough to cause damage to the glass if the shot rate was high enough, and even non-damaging levels of heat can change the absorption and emission properties enough that a subsequent laser shot will have drastically different mode quality.[40] This renders the shot at best inconsistent with previous ones and at worst potentially damaging to consecutive laser optics. As a result, the biggest lasers had hours of downtime between firing at full power.

As laser energy increased and pulse duration decreased over the years, the repetition rate also crept higher on these large systems. This was initially achieved with improved cooling of the gain medium, which must be done with some concern towards parasitic lasing that will build up if the cooling fluid is not index matched closely to the crystal. ${ }^{6}$ The ubiquity

${ }^{6}$ Parasitic lasing occurs when spontaneous emission of the pumped medium results in light travelling 


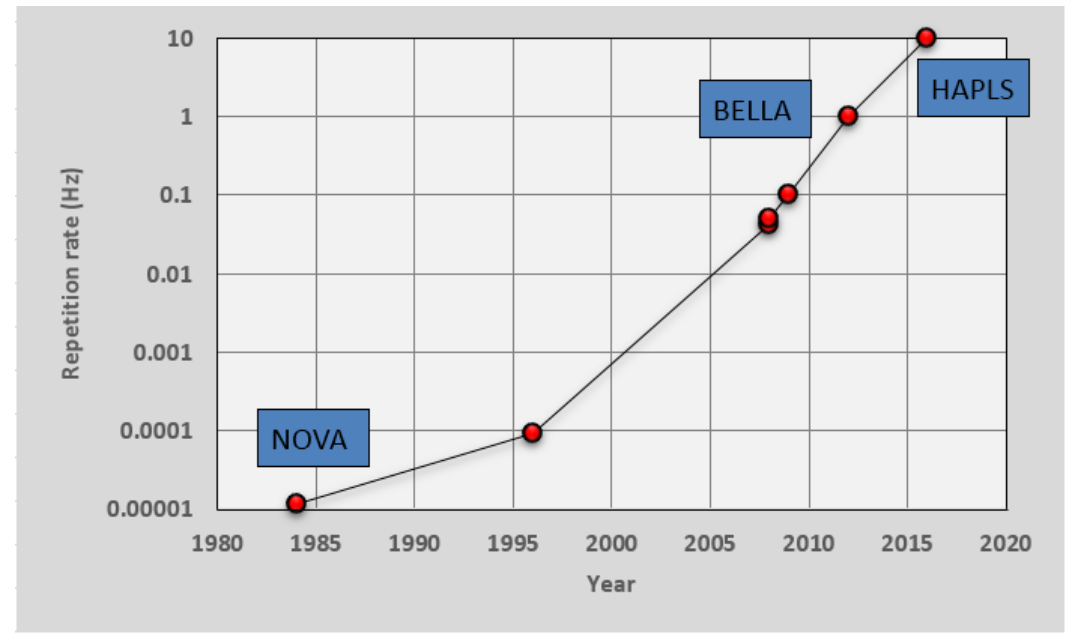

Figure 3.1: Chart of laser shot rate in $H z$ as a function of time. Here a few key lasers are indicated, including the BELLA facility at Lawrence Berkeley National Laboratory (LBNL) and the HAPLS laser being constructed for the Extreme Light Infrastructure (ELI) project in Europe.

of Ti:sapphire gain crystals also helped increase repetition rate due to its high damage threshold and superior thermal conductivity compared to many types of glass.[14]

The result was an influx of higher repetition rate laser systems over the past decade or so. Shot rates of once per minute or even once per second were achievable at high intensities after 2010, and the upcoming ELI facilities will have ten petawatt systems running at 10 $H z$ before 2020. These lasers have been and are being built with the promise of a myriad of applications that demand high particle or radiation yield, or experiments that require large amounts of data.[41]

While the technological increase in terms of laser shot rate has climbed fast, the ability to deliver targets optimally at these repetition rates has lagged behind. What follows is a discussion of the state of the art in terms of high repetition rate targets prior to the work introduced here, with an emphasis on their utility and detriments for various applications.

along the transverse direction of the gain crystal. If the crystal housing is reflective, or if the surrounding cooling fluid has a different index of refraction than the gain medium, Fresnel reflections will ensure some light travels back through the crystal and a cavity of sorts can be set up transversely. This robs the main, longitudinally traveling pulse of pump energy. This can be solved by index matching, where the cooling fluid has both a component to match its index of refraction as closely as possible to that of the gain medium (thereby reducing Fresnel reflections from their interface) as well as a dye that absorbs the desired frequency of radiation to prevent it from experiencing gain by traveling back through the pumped medium. Unfortunately the index matching fluid can often be a hazardous material as it is with the di-iodomethane $(n=1.741)$ used in Ti:sapphire $(n=1.76)$ amplifiers. 


\subsection{Gas jets}

Gas jets have been used for years in low-intensity laser experiments, especially in the production of attosecond pulses.[42] Often a noble gas will be sprayed with some backing pressure (e.g. 100 Torr) through a nozzle within the target chamber.[43] The density of gas molecules available for laser interaction can be varied by changing the backing pressure of the gas or simply by translating the nozzle with respect to the laser. The noble gasses often used are relatively benign to sensitive laser optics in the system, and can be pumped out of the chamber with the standard turbomolecular pumps used for initial evacuation (and that typically remain on to maintain vacuum level). The exact gas molecules can change depending on the desired physics to be studied.

Gas or liquid sprays have also been investigated for high intensity laser applications like ion acceleration, again with the hopes of having a high repetition rate interaction for increased yield. Karsch et al.[44] used $\mathrm{D}_{2} \mathrm{O}$ droplet targets to accelerate deuterons for producing fusion energy neutrons in a converter target. These droplets were formed consistently with $20 \mu \mathrm{m}$ diameters from a piezoelectrically modulated $10 \mu \mathrm{m}$ diameter nozzle. This setup design was studied for some time, showing enhanced conversion efficiency and yield from $\mathrm{D}_{2} \mathrm{O}$ droplets compared to cluster targets.[45] Somewhat mono-energetic ion acceleration was also observed, potentially due to the spherical shape of the target.[46] Later analysis[47] suggested that droplet targets have a larger proton or deuteron reservoir than the contaminants typically accelerated from a planar target, as well as a smaller surface area for Target Normal Sheath-field Acceleration (TNSA) to occur. This allows for a higher electron sheath density and therefore longer lasting sheath field (and hence more acceleration time) than on planar targets. Furthermore, droplet targets interacting with the laser are automatically mass-limited targets,[45] a subject of some study within the field, where the relatively small target volume can be heated uniformly during laser interaction. The result of such heating is more consistent, hotter target interactions. Liquid sprays can potentially be formed without the typical water vapor and hydrocarbon contaminant layer that accumulates on targets[48] due to the rapidity of the droplet formation and travel to the laser focal region.

As a downside, the three dimensional expansion of a droplet target leads to faster cooling which ultimately results in a lower cutoff energy for these targets as opposed to those with planar geometries.[47] Their spherical nature also has targets accelerate ions via TNSA from their entire surface (rather than just the back side as with a planar target)-the result is less overall energy into the forward-accelerated ions and larger effective divergence due to the isotropic acceleration.[28] Additionally, the charging of one droplet compared to its immediate neighbors due to leaving electrons seems to effect the acceleration physics at least inconsistently, and at times detrimentally.[49] 
A further uncertainty from droplet targets comes from the details of their interaction with the laser focus. Typically foci are on the order of several to several tens of $\mu m^{2}$ : at certain droplet densities and relatively large droplet diameters this means there could be an imperfect alignment of incoming laser to droplet target, or worse no droplet within the laser focus as it arrives. These near misses tend to result in a broader accelerated ion energy spectrum,[50] while the full misses can occur as frequently as one in four shots on certain experimental setups.[51]

Some effort has been applied to producing droplet diameters on the order of a few $\mu m$ and below. [51, 52] These efforts have improved upon early experimental setups to the point that at least some of the droplet cloud can consist of sub- $\mu m$ diameters with moderate densities $\left(\right.$ near $\left.10^{18} / \mathrm{cm}^{3}\right)$.[52]. A $40 \mathrm{fs}, 5 \times 10^{19} \mathrm{~W} / \mathrm{cm}^{2}$ experiment produced quasi-monoenergetic protons from such a spray, with energies of $3 \mathrm{MeV}$.[53] Despite these advances, for many applications this low proton energy and isotropic emission is non-ideal.

The possibility of high rep rate ion acceleration is enough to recommend the use of droplet targets for some experiments. However, the difficulties in decreasing droplet size down to the few and sub- $\mu m$ diameters advantageous to TNSA and other acceleration mechanisms as well as the disadvantages of spherical geometry to acceleration render them non-optimal for many applications. Furthermore, only low- $Z$ materials have been used in the formation of these droplets, which prevents their utility for the more traditional metallic target experiments of the high intensity laser field such as high yield electron acceleration, bremsstrahlung x-ray production, and gamma ray generation.

\subsection{Tape targets}

Another rapid target delivery mechanism comes in the form of a moving tape target. Here a spool of metallic or plastic tape is wound from one spool to another, much like in a common VHS tape mechanism. This presents a flat, vertically moving target strip between the spools for laser interaction, as shown in Fig. 3.2. In the design of Nayuki et al.[54] a $5 \mu m$ thick copper strip is moved with two stepper motors, the first of which controls the torque while the second winds the tape. The strip is $2 \mathrm{~cm}$ wide, which is necessary to provide enough strength such that the tape remains intact after being hit by the laser pulse.

Tape targets have also been proposed or used on other laser systems, most notably and recently as a plasma mirror surface.[55] The ribbons can be made metallic, striking at applications not possible with the droplet targets of the previous section. These tapes also require no external plumbing or chamber setup beyond the electronics capability to control the motors from outside vacuum. As planar targets these tapes will benefit from the optimal geometry for TNSA and other acceleration mechanisms. They cause less strain to the vacuum pumping system than any liquid or gas targets, and are capable of being 


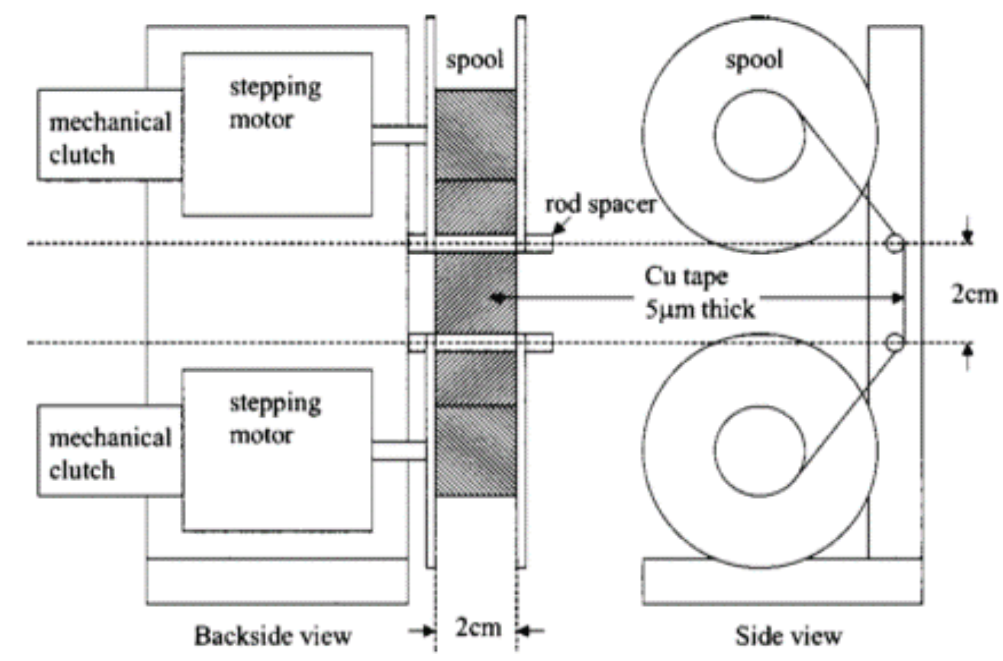

Figure 3.2: Moving tape target concept schematic. Here two motors spin a thin strip of metal or plastic between two spools, leaving a region of vertically-moving planar target strip in between to be shot by the laser. From [54].

long term single-shot targets if high repetition rate shots are not available.

Unfortunately the mechanism developed for use in Japan suffered from some longitudinal positioning jitter on the order of $30 \mu \mathrm{m}$ or more. [54, 56] This severely hinders their utility for high repetition rate shots, as this large positioning inconsistency can result in many orders of magnitude difference in shot-to-shot intensity on a short $F / \#$ system. Additionally, the tape itself cannot be made thinner than a several $\mu m$, so this device is not capable of reaching the few and sub- $\mu m$ thicknesses advantageous to ion acceleration mechanisms. Even several $\mu m$ thick tapes need to be relatively wide to allow sufficient structural support to withstand the laser impact.

Furthermore, pushing this device to higher rep rates necessitates increasing the tape spinning speed to a point where thicker tapes are required to overcome the stresses inherent to the process-this makes the technology not scalable to the repetition rates of droplet sprays, for example. Finally, it is suggested[54] that a debris shield be used to protect sensitive surrounding optics in the chamber, due both to the metallic projectiles resulting from intense laser interaction (which will only be more prevalent at higher repetition rates) and to the possibility of catastrophic damage from a ruptured tape.

\subsection{Water jets}

Another classification of liquid laser targets is water jets. These are distinct from the previously discussed droplet sprays in that the volume of liquid remains as a steady stream 

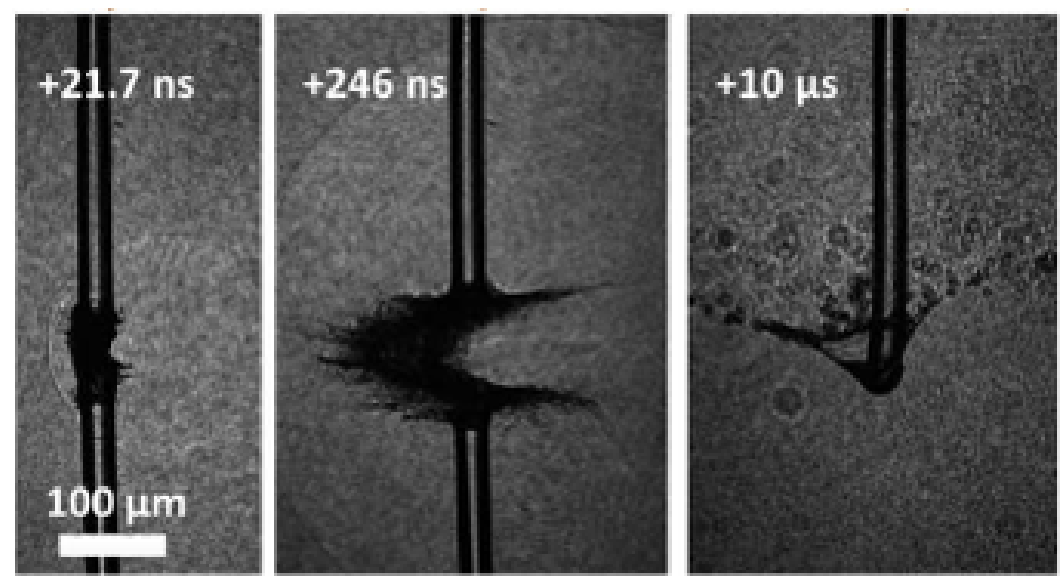

Figure 3.3: Water jet ejected at speed from a nozzle, from [57]. This jet is tens of $\mu m$ in diameter, experiencing intense laser interaction from $1 \mathrm{kHz}$ pulses.

until laser interaction. This technique often relies on high pressures to force the jet fluid through a small enough nozzle to allow a thin stream to emerge at high velocity. A typical jet image can be seen in Fig. 3.3.

Jets in this fashion have been used more traditionally in photoelectron spectroscopy experiments, [58] where an incident laser or x-ray beam can be used to excite photoelectron emission in a material dispersed within the water. In such experiments it is important that the jet diameter remain small to minimize absorption or scattering of emitted photoelectrons on their way out of the bulk volume. As such diameters down to and below $10 \mu \mathrm{m}$ have been utilized.

Recently these water jets have been applied to high intensity laser experiments focused on electron or ion acceleration.[59,60] Specifically, electrons have been accelerated at $k H z$ repetition rate from a $>10 \mu \mathrm{m}$ jet of water using a $I \sim 10^{18} \mathrm{~W} / \mathrm{cm}^{2}, 30 \mathrm{fs}$ laser. [57] The electrons or ions accelerated from such a jet benefit from the high repetition rate possible with the water flow speeds and somewhat from the cylindrical geometry of target interaction. This shape is not as optimal for TNSA as a true planar target but has significant advantages over spherical droplets. Jets can even be forced to be planar for a small region by modifying the nozzle aperture to mimic a laser dye jet, as will be discussed in Chapter 11.

Water jet systems are currently still not optimal for ion acceleration and other high intensity applications due to the inability to reduce the jet thickness below several $\mu \mathrm{m}$, though some schemes used in the photoelectron spectroscopy community could prove useful in the near future. [61] Additionally, the constituents of the water jet are difficult to change if different acceleration physics are desired-in all cases some amount of $\mathrm{H}_{2} \mathrm{O}$ will be present in the laser focus. Even if metal can be placed in the fluid volume in the form of nanoparticles or a dissolved material, the lighter hydrogen and oxygen ions can have detrimental effects 
on heavy ion processes.[62]

Another type of cylindrical liquid jet target is cryogenic hydrogen.[63] These targets have the advantage of being high rep rate, low- $Z$, and relatively low vapor pressure. However, the cryogenic requirement can present difficulties with the experimental setup, especially with regards to alignment stability (although this is being improved currently). A further difficulty for these and other jets with regard to ion acceleration and related applications lies in the cylindrical geometry of the target. The cylindrical targets have similar if somewhat mitigated issues to spherical droplets with regard to target cooling and non-directed ion sprays.

Perhaps the chief obstacle for the use of water jets for high intensity laser experiments lies in the effect these relatively large volumes of water have on the target chamber environment. Even with protective chilled shrouds and liquid reclamation plumbing, an increased level of water vapor will be present in the chamber and eventually on sensitive focusing and alignment optics. To combat this a differential pumping technique is often implemented where the jet functions within a somewhat closed-off inner section of chamber that has separate turbomolecular pumps to reduce vapor contaminating the outer chamber. With this setup and sufficiently small inner chamber openings it is possible to keep the outer chamber at normal operating vacuum levels while the inner chamber remains closer to the water vapor pressure of 20 Torr.

Even differential pumping does not resolve the issue for short pulse systems, however, due to the phase disorder from short pulse propagation through this water vapor volume. The breakup integral, or B-integral, describes the accumulation of nonlinear phase effects from a short laser pulse traveling through any non-vacuum material:

$$
B=\frac{2 \pi}{\lambda} \int n_{2} I d x<1
$$

where $\lambda$ is the laser wavelength in $\mu m, n_{2}$ is the second order nonlinear index of refraction in $\mathrm{cm}^{2} / W$, and $I$ is the intensity in $W / \mathrm{cm}^{2}$. Essentially the amount of material the laser passes through with a non-unity $n_{2}$ is summed, and if this resulting value is near or greater than 1 the accumulated nonlinear phase will cause the beam to self-focus, or break-up, into a number of beamlets. These beamlets will in general degrade the laser mode quality at focus and reduce the maximum intensity drastically as well as potentially damage other optics further along the laser chain. Additionally, a pulse stretching effect will occur whereby very short pulses will increase in duration due to travelling through this non-vacuum medium, again reducing the laser intensity significantly. As a figure of merit, a $30 \mathrm{fs}$ pulse traveling through $10 \mathrm{~cm}$ of fused silica will leave at $330 \mathrm{fs}$, while a $10 \mathrm{fs}$ pulse will be stretched to $100 \mathrm{fs}$ from traveling through only $1 \mathrm{~cm}$ of glass.

The negative effects of short pulse propagation through the low density vapor area around a target jet severely limit this target design for high repetition rate intense laser 
experiments. A material with lower vapor pressure than water must be used for such applications.

While each of these methods has been used for certain experiments and applications, they all have detrimental aspects preventing widespread use. Subsequent Chapters of this work will describe how liquid crystal films remove many or all of these negative aspects while maintaining the optimum parameters for experiments. 


\section{Chapter 4 \\ DESIGN AND CONSTRUCTION OF Petawatt-Scale Scarlet Laser FACILITY}

\subsection{Overview}

The number of mid-tier high terawatt and petawatt scale laser facilities has increased greatly over the past 10 years. $^{7}$ While many of these facilities were originally established to provide fundamental science support for the larger systems like those at the National Ignition Facility (NIF) or Laboratory for Laser Energetics (LLE), many more have since focused exclusively on the growing number of applications available to a high intensity laser experimental facility. These mid-tier systems typically have the advantage of firing much more rapidly than larger lasers, enabling more frequent experimental shots and allowing research into some applications impossible with lower shot rates.

What follows is an introduction to one such system: the Scarlet laser facility at the Ohio State University. After its most recently upgrade the system has a peak power to $400 \mathrm{TW}$. Scarlet is a Ti:sapphire based ultra-short pulse system that delivers $15 \mathrm{~J}$ in $30 \mathrm{fs}$ pulses to a $5 \mu \mathrm{m}$ full width at half maximum (FWHM) focal spot, resulting in intensities exceeding $10^{21} \mathrm{~W} / \mathrm{cm}^{2}$. The laser fires at a repetition rate of once per minute and is equipped with a suite of on-shot diagnostics detailed here, allowing for rapid collection of experimental statistics.

\subsection{Introduction}

The era of Chirped Pulse Amplification (CPA)[64] has seen consistent advancement in both the repetition rate of ultra-intense laser systems and in the energy they deliver to a target.

\footnotetext{
${ }^{7} \mathrm{~A}$ map of the current facilities that have peak intensity within three orders of magnitude of the maximum recorded to date can be found on the website of the International Committee on Ultra-High Intensity Lasers, here: http://www.icuil.org/events-a-activities/laser-labs.html.
} 
Since the first petawatt-scale laser experiments in the late 90 's[65] a number of table-top laser facilities have emerged at large institutions around the world[35, 66, 67] with intensities today routinely reaching $10^{20} \mathrm{~W} / \mathrm{cm}^{2}$ and above. These intensities allow the study of a wide field of applications including ion acceleration and its applications of neutron radiography[3] and proton cancer therapy[5], as well as energetic electron acceleration for generating $\mathrm{x}$ ray [6, 7] and positron beams[8] for laboratory astrophysics, to name a few. Still higher intensities promise experiments on radiation reaction[68] and relativistic transparency[39], among others.

The Scarlet laser facility at the Ohio State University is a $400 T W$ short pulse laser system, delivering $12 \mathrm{~J}$ of energy with $30 \mathrm{fs}$ pulse duration within a spot size of $5 \mu \mathrm{m}$ FWHM. The resulting intensity is in excess of $10^{21} \mathrm{~W} / \mathrm{cm}^{2}$. Scarlet has been designed to facilitate rapid data collection and analysis, bringing to bear a suite of on-shot diagnostics for energy, spectrum, pulse width, contrast, and wavefront quality, all available at the laser shot repetition rate of once per minute. A number of system highlights are detailed here, in particular the implementation of a parabolic mirror telescope that eliminates the post-pulse and scattered light detriments of traditional transmissive element image-relay designs.

\subsection{Laser design}

\subsubsection{Pulse generation and preamplification}

Scarlet uses dual chirped pulse amplification to facilitate a Cross-Polarized Wave (XPW)[34] contrast enhancement system. The first element in the front-end is a Femtolasers Femtopower(R) Pro unit consisting of a Ti:sapphire Kerr lens modelocked oscillator, ${ }^{8}$ glass stretcher, nine-pass amplifier, and prism compressor. The oscillator is pumped with a Femtolaser SynPro Laser Quantum pump laser that sends $4.0 \mathrm{~W}$ of continuous wave $(\mathrm{CW})^{9}$ light to a $3 \mathrm{~mm} \times 1 \mathrm{~mm}$ Brewster cut Ti:sapphire crystal. The resulting $75 \mathrm{MHz}, 9 \mathrm{fs}$ pulse train is sent through a glass stretcher ${ }^{10}$ before amplification in a nine-pass Ti:sapphire

\footnotetext{
${ }^{8}$ Kerr lens modelocking is a method by which a series of short pulses can be produced in a laser oscillator. The optical Kerr effect is a change in the refractive index of a medium due to a large electric field, so a laser cavity can be designed such that only pulses with sufficient intensity to produce this Kerr effect can make full round-trips through the oscillator. Such a cavity typically produces standing longitudinal waves with wavelengths that are multiples of twice the cavity length $L$. In a Ti:sapphire oscillator there can be on the order of $10^{5}$ such modes, but with a random distribution of phases. It is the goal of modelocking to bring these many longitudinal modes to have the same phase such that their intensities add into one pulse with sufficiently high frequency content to make it short on the femtosecond scale. The details of Ti:sapphire oscillators and the principle of modelocking can be found in a number of texts.[69]

${ }^{9}$ Laser cavities can be turned on and off in various optical or electronic ways to generate pulses of light of various durations. If none of these is applied, a so-called "free-running" laser cavity will constantly generate output light, as long as a population inversion is consistently maintained in its gain medium.

${ }^{10}$ Pulse stretching is a consequence of sending multiple frequencies of light through a dispersive element. This can be a diffraction grating, as will be mentioned later, but for short pulses the group velocity dispersion induced by different frequencies traveling through a small piece of glass is enough to cause a significant spread of instantaneous frequencies with respect to time. This quality of the pulse is known as chirp, since a changing
} 
amplifier pumped by $8 W$ of 200 ns pulse light from a Jade I pump laser. After 4 passes a Pockels cell ${ }^{11}$ unit takes the beam to $1 \mathrm{kHz}$ repetition rate for further amplification and eventually to a four-prism double pass compressor system. The end result is $800 \mu \mathrm{J}, 25$ $f s$ pulses with a $55 \mathrm{~nm}$ bandwidth centered on $800 \mathrm{~nm}$ delivered at a repetition rate of 1 $k H z$. The distance between the compressor prisms can be modified to vary the front-end pulse width when desired, or to optimize the spectral shape for the XPW process.

This first stage of amplification generates sufficient intensity for XPW pulse cleaning, which is necessary due to the high amount of amplification achieved in the Femtopower unit. The XPW takes advantage of the cubic response to incident intensity for the cross polarized wave generation nonlinear optical process. The nonlinear elements are two $\mathrm{BaF}_{2}$ crystals with holographic [011] cut situated between crossed polarizers. Since the XPW process scales with the cube of the intensity, prepulses and optical noise from the oscillator will generate weaker cross-polarized signals than the main pulse, resulting in intensity contrast improvement of $10^{-4}$.

After this contrast improvement the pulse is sent through a second stretcher in preparation for main amplification. A new stretcher was designed for the Scarlet upgrade based on the Perry-Banks schematic,[70] which uses as its only diffractive element a 12" x 4" gold-coated grating with a 12" x 1" mirror stripe along its center. A 16" diameter concave mirror with focal length $240 \mathrm{~mm}$ steers the beam to different heights on this diffraction grating such that two grating hits occur on the first pass through the system and the other two grating hits occur after retro-reflection. A roof mirror pair sends the partially stretched and spatially separated beam back through the system at a slightly different height in order to double the temporal stretching and recombine the beam spatially. This striped grating design allows for simpler alignment and increased stability of the stretcher. The design was optimized using the optical ray-tracing software FRED for maximum bandpass $(140 \mathrm{~nm})$, maximum stretching factor to reduce B-integral, as well as for minimal angular chirp of the stretched pulse. Here and elsewhere in the system careful consideration has been given to the reduction of nonlinear phase accumulation, or B-integral, which can cause filamentation of the focused beam. The B-integral value after the entire Scarlet laser chain is only 0.5. This second stretcher takes the pulse duration from around $25 \mathrm{fs}$ to $800 \mathrm{ps}$. A Phasics wavefront sensor was utilized for fine adjustment of the stretcher geometry to minimize phase error before amplification. 


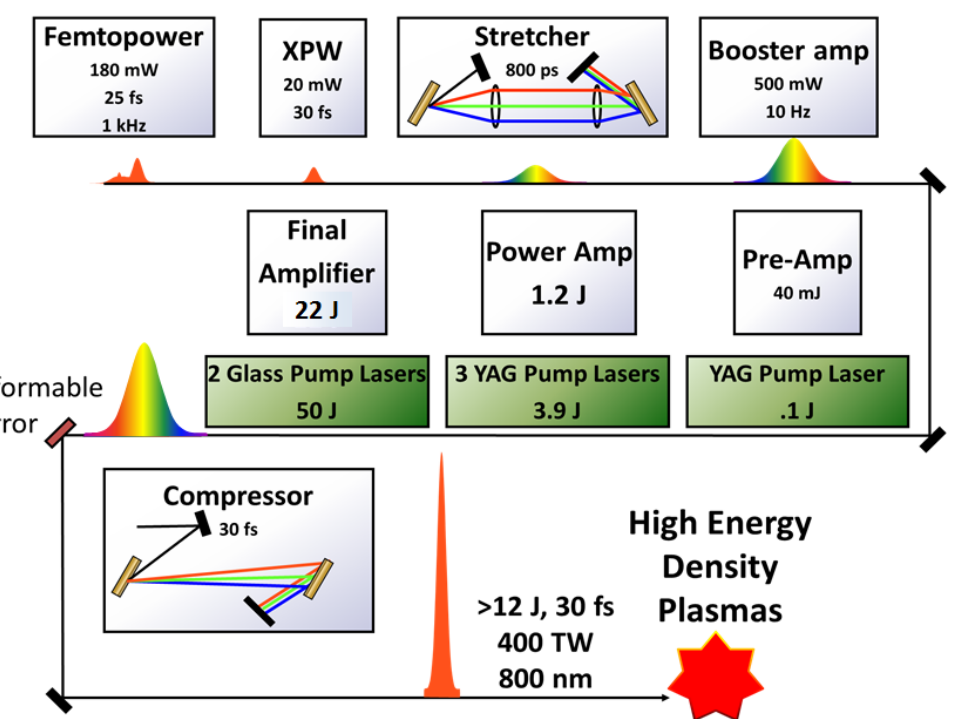

Figure 4.1: Schematic of Scarlet pulse generation and amplification. Green boxes indicate pump lasers and their parameters, and gray boxes are the short pulse specifications.

\subsubsection{Amplification}

After stretching the pulse undergoes four stages of amplification including the new final amplifier, as shown in Fig. 4.1. The optical layout of Scarlet has been designed to allow for image-relay between the last two amplifiers, preserving beam quality over the meters of air propagation between these elements. The relay optics are chosen such that the beam mode at the last pass of one amplifier crystal is imaged directly to the front face of the next amplifier, at the same time magnifying the beam by the correct amount to protect optics from damage. Amplifier pump beams have also been image-relayed to preserve pump mode quality and ensure uniform amplification across the beam. Additionally, the beam mode can be viewed after each amplification stage to preserve beam quality and diagnose problems. Isolation systems in the form of Faraday isolators ${ }^{12}$ and Pockels cells have been placed at frequency with respect to time is reminiscent of birdsong.

${ }^{11}$ Pockels cells operate on the principle that certain optical media can have a birefringence induced by an external electric field. In this way a Pockels cell is a waveplate switchable on electronic timescales-several tens of $n s$, typically.

${ }^{12}$ Faraday isolators operate by rotating the polarization components of light passing through a crystal with a large external magnetic field-this is the magneto-optical effect, or Faraday effect. A Faraday rotator has a specific crystal length and magnetic field to shift incoming light by a set amount. Critically, this polarization rotation depends on the direction of light through the crystal, such that a backward propagating beam will experience the same rotation once again rather than a negation of the original rotation. Placing polarizers before and after such a rotator creates a Faraday isolator, where the additional rotation from a backward propagating beam will cause it to be rejected by the polarizer, thus protecting optics further up the laser chain. 
critical points between amplifiers to protect the beam from back-reflections propagating through the system.

The first amplification stage is a two-pass booster amplifier pumped with $14 \mathrm{~W}$ of power diverted from the Jade I pump laser which also supplies energy for the pre-XPW nine-pass amplifier. This brings the beam energy from about $10 \mu \mathrm{J}$ to $600 \mu \mathrm{J}$ at $1 \mathrm{kHz}$ before a Pockels cell reduces the pulse repetition rate to $10 \mathrm{~Hz}$. This Pockels cell and a Faraday isolator provide back-reflection isolation to protect earlier optics. Next a fivepass pre-amplifier pumped by $150 \mathrm{~mJ}$ from a Continuum Powerlite Plus DLS $5 \mathrm{~ns}, 1.5 \mathrm{~J}$ max pump laser brings the short pulse energy up to $45 \mathrm{~mJ}$. A four-pass power amplifier is then pumped by the remaining $1.4 \mathrm{~J}$ of Powerlite energy as well as $1.4 \mathrm{~J}$ each from two Thales Neodymium:Yttrium Aluminum Garnet (ND:YAG) SAGA $5 n s, 1.4 J$ lasers. All of these pumps are image-relayed to the $20 \mathrm{~mm}$ diameter, $20 \mathrm{~mm}$ thick Ti:sapphire crystal for optimum beam quality. The $1.4 J$ short pulse output serves as Scarlet's lowpower $10 \mathrm{~Hz}$ operation mode. For petawatt mode, a Pockels cell picks the pulse train down to a $1 / \mathrm{min}$ repetition rate before it propagates through a $100 \mu \mathrm{m}$ pinhole spatial filter for back-reflection protection. The spatial filter telescope uses two custom air-spaced apochromatic lenses designed at Ohio State using OSLO optical software and fabricated by Rocky Mountain Instruments to minimize various aberrations and ghost focusing. ${ }^{13}$

The last amplification step is the three-pass petawatt amplifier, the centerpiece of which is a $70 \mathrm{~mm}$ diameter, $30 \mathrm{~mm}$ thick Ti:sapphire crystal purchased from Crystal Systems that amplifies the $45 \mathrm{~mm}$ diameter short pulse beam. The crystal is anti-reflection coated for $527 \mathrm{~nm}$ and $800 \mathrm{~nm}$ with $94 \%$ pump beam absorption in a single pass and transmitted wavefront distortion of less than $\lambda / 4$. This crystal is pumped by two custom Continuum Nd:glass pump lasers with multiple amplification stages of their own including an oscillator, Neodymium:Yttrium Lithium Fluoride (ND:YLF) pre-amplifier, beam-shaper, then a 16 $\mathrm{mm}$ Nd:glass amplifier followed by a $25 \mathrm{~mm}$ amplifier. This results in two beams per laser each with $25 \mathrm{~J}$ of $1053 \mathrm{~nm}$ light. These beams are frequency doubled in uncoated Potassium Di Phosphate (KDP) crystals to produce $12.5 \mathrm{~J}$ of $527 \mathrm{~nm}$ light per arm, for a total of 50 $J$ of pump energy at once per minute sent to the amplifier crystal. Retro reflecting mirrors recycle the unabsorbed pump beams by steering them back toward the crystal. The final amplification stage fires once per minute, setting the overall repetition rate of Scarlet in petawatt mode. These pump beams are image-relayed from the back of the second harmonic

\footnotetext{
${ }^{13}$ Ghost foci are produced from the small amount of light that will be reflected from an anti-reflection coated surface of a transmissive optic. Such coatings are often good to the $99.95 \%$ transmission level, but on a high-power system even the small amount reflected can be dangerous. This is especially true for ghost reflections from lenses, which will focus in different locations depending on what lens surface the light is reflecting from and therefore how many times it passes through the lens. Achromatic lenses are typically comprised of two pieces of glass with different materials and curvatures, resulting in four surfaces (in an airspaced achromat) for unwanted reflections and a number of possible foci locations. It is all too uncommon for such ghost foci to be forgotten when designing a new optical layout, often with expensive consequences.
} 
generation crystal to the Ti:sapphire amplifier crystal face. The amplifier has been designed with mode imaging systems on each of the four pump beams and for each pass of the short pulse, allowing on-shot observation and collection of all relevant beam modes-the short pulse beam modes at each pass are shown in (Fig. 4.2).

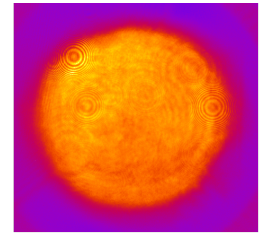

$1^{\text {st }}$ pass $7 \mathrm{~J}$

$48 \mathrm{~J}$ of pump energy/pulse with an input of $1.3 \mathrm{~J}$

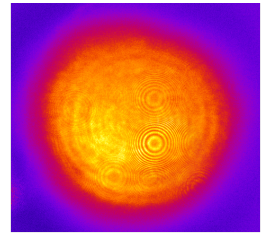

$2^{\text {nd }}$ pass $15 \mathrm{~J}$

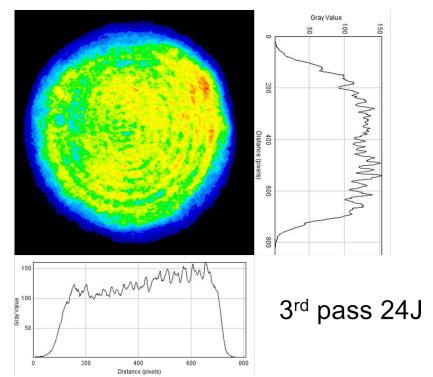

Figure 4.2: Shown here is the short pulse mode for each of the three passes within the final amplifier. Each short pulse pass and pump arm of the final amplifier can be monitored in real time with mode imaging cameras to ensure optimum pumping configuration and to protect from the development of hot spots within the beam mode.

The petawatt amplifier crystal mount was designed and built at Ohio State. It uses diiodomethane $\left(\mathrm{CH}_{2} \mathrm{I}_{2}\right)$ with $n=1.736$ index matching fluid (Cargille Laboratories) and laser dye HITC (Exciton) to absorb any Amplified Spontaneous Emission (ASE) that propagates transversely through the crystal to enter the outer cladding. The mount also has the capacity for cooling lines, although these are not necessary with the current once per

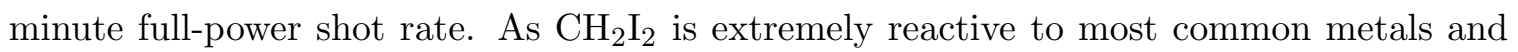
organics, care was taken in designing the crystal holder to safely use this index matching fluid. Figure 4.3 shows the small signal gain curve for the final amplifier. Full-energy (uncompressed) shots of $>22 J$ are output from the amplifier and sent to the target area for final compression.

\subsubsection{Compression}

After the final amplifier the beam is expanded to its full $150 \mathrm{~mm}$ diameter by another OSU custom-designed $150 \mathrm{~mm}$ achromatic lens and a $200 \mathrm{~mm}$ glsOAP mirror with an $8 \mathrm{~m}$ focal length. As with the rest of the system this telescope is image-relayed, transferring the beam mode at the back of the last pass of the petawatt amplifier crystal to the fourth grating hit in the compressor. This image plane choice minimizes any phase aberrations that will occur by the final grating hit, at which point the beam is fully compressed and therefore most dangerous to the optic. Using an Off-Axis Parabola (OAP) as this final relay optic also 


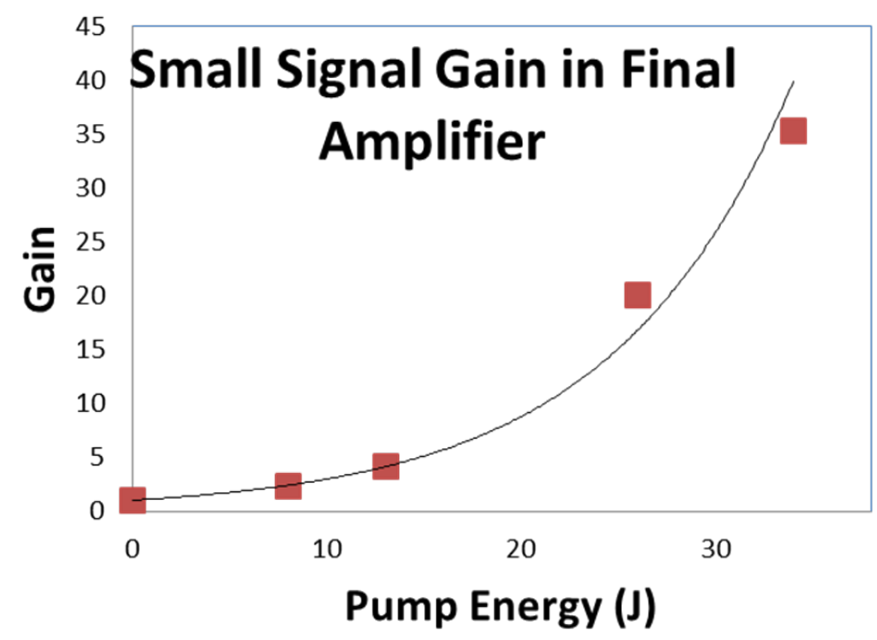

Figure 4.3: Small signal gain data for the final amplifier as the pump energy is increased up to its maximum of $50 \mathrm{~J}$ of $527 \mathrm{~nm}$ light. The curve is the theoretical small signal gain model, $[40]$ to which the measured data conforms.

reduces radial group velocity delay, which improves the beam compression and eventually the mode quality.

Alignment of the OAP is achieved by inserting a beamsplitter near the focus of the image-relay, well before the OAP. Light reflecting from this splitter is imaged to a camera, while the transmitted light is collimated by the OAP and then immediately retro-reflected to be focused again by the optic and sent via the near-focus beamsplitter to the same camera as the input light. By viewing these two beams (one from the input to the OAP and one that has reflected from it twice) on one camera it is possible to adjust the angles and position of the OAP with fine precision and iterate away any aberration due to misalignment. The same system can be used to align the gratings in Littrow reflection mode. ${ }^{14}$

The compressor chamber at OSU is a $9^{\prime} \times 5^{\prime}$ cylindrical chamber pumped by a Leybold SP250 screwline roughing pump and two Oerlikon turbomolecular pumps. It is capable of achieving vacuum levels of $10^{-8}$ Torr, and can be vented with filtered, humidity-controlled air when desired.

The OSU gratings were designed by Plymouth Grating Laboratories, and are $340 \times$ $420 \mathrm{~mm}$ and $560 \times 420 \mathrm{~mm}$ in size. The gratings are made using a modification to the Nanoruler[71] metrology and sputtering system that allows for a conformal gold coating to be applied to a ruled fused silica substrate. This design was shown during damage testing described in Chapter 5 to exhibit 20\% higher short-pulse damage threshold properties due

\footnotetext{
${ }^{14}$ Littrow angle is that angle for which the first order diffraction is sent exactly back along the incoming light path.
} 
to this conformal coating than those of traditional coating techniques.[72] The measured damage threshold of this grating type was $>200 \mathrm{~J} / \mathrm{cm}^{2}$, and in Scarlet they are operated with a factor of 2 safety at $100 \mathrm{~J} / \mathrm{cm}^{2}$. Another benefit of this coating style revealed in damage testing experiments was that the fused silica groove structure remained intact upon damage, indicating that if necessary the grating could be stripped and re-coated unlike standard gratings.[72]

A genetic algorithm has been developed to determine the optimum grating separation distance given the amount of dispersive elements in the Scarlet laser chain. This code is based on minimizing the root mean square (RMS) phase error to all orders rather than minimizing individual Taylor series orders.[73] The spectrum at various points in the Scarlet amplification chain can be seen in Fig.4.4, where the legend organizes the spectra vertically by their location in the system (with the top being the earliest measurements). Here the heights are not normalized to any value and should be ignored. Of note is the strong spectrum modulation produced by theXPW device (due to its third order effect), as well as the shift to longer wavelengths and bandwidth narrowing as the pulse travels through amplifiers. These effects are due to the positively chirped pulse, which has longer wavelengths traveling in front of shorter ones: as such the longer wavelengths encounter the most pump energy in the amplifier crystals, whereas the later parts of the pulse see a slightly depleted gain medium. This gain narrowing and shifting must be taken into account for proper pulse compression, and is modeled and optimized in the Scarlet system.

Manipulations of compressor grating motion as small as $5 \mu \mathrm{rad}$ angularly and $5 \mu \mathrm{m}$ translationally are possible due to in-house designed and constructed mounts for all optics in the compressor and target chambers. These mounts have all been connected to the Scarlet facility internal network system, which allows for motion control from a web interface from any computer within the lab. Additionally, relevant parameters like the grating separation distance are encoded so that the setting is remembered and can be reproduced and monitored for fine compressor alignment. If desired, the pulse width can be tuned larger than the minimum value of $30 \mathrm{fs}$ by using these motion control on the grating separation. The Scarlet system as a whole can be seen in Fig. 4.5.

A thin $(4 \mu \mathrm{m})$ nitrocellulose pellicle is situated at Brewster's angle between the compressor and target chambers to guard against ballistic particulates and general contamination from any target chamber experiments.

The operational modes and their beam parameters are shown in Table 4.1. Scarlet can operate in a $10 \mathrm{TW}$ mode with its final amplifier turned off if $10 \mathrm{~Hz}$ repetition rates are required. 


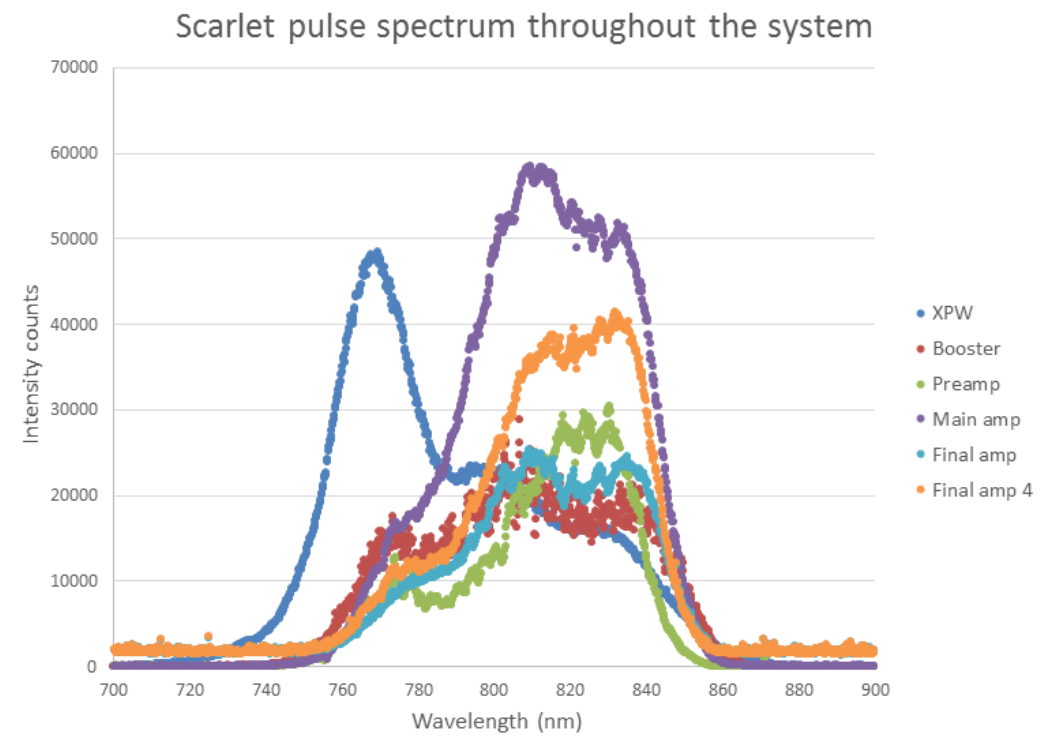

Figure 4.4: Measurements of the short pulse spectrum at various amplification stages within Scarlet. The heights are not normalized, and vary only due to the proximity of the spectrometer fiber to the scatter surface during measurement. There is a shift toward longer wavelengths due to these being slightly preferentially pumped because the positive pulse chirp sends longer wavelengths through the fully energized crystals first.

\subsection{Diagnostics capabilities}

\subsubsection{Laser diagnostics}

Laser parameters that sensitively effect experiments are tracked on a shot-to-shot basis or on-demand. On-shot laser diagnostics utilize a $1 \%$ leak-through on the final flat mirror in the target chamber. This full-aperture leak-through is analyzed outside the target chamber in a setup shown in Fig. 4.6. On-shot laser diagnostics include an equivalent-plane focal spot analyzer, spectrometer, energy meter, and autocorrelator, calibrated for differences between the diagnostic location and the experimental target location when necessary. A second autocorrelator, in-vacuum, allows precise measurement of the pulse width on-demand by inserting a pickoff into the beam path within the compressor chamber. A scanning thirdorder cross correlator[74] developed in-house is used to measure picosecond-scale contrast using Scarlet in $10 \mathrm{~Hz}$ mode.

\subsubsection{Target diagnostics}

The Scarlet target chamber is a 38-port, 76" diameter cylinder 60" tall with domed lid, based on the TITAN chamber at Lawrence Livermore National Laboratory (LLNL), only $2 / 3$ the size. The chamber is pumped to rough vacuum levels with the same screw line 


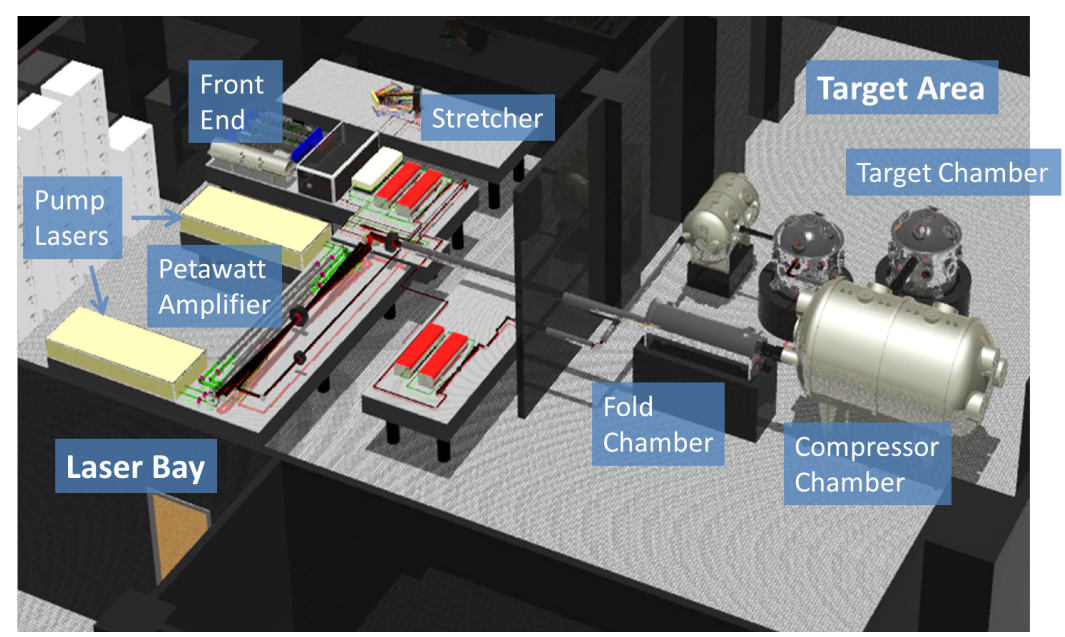

Figure 4.5: Schematic of the entire Scarlet system including the pulse generation and amplification and target areas.

Table 4.1: Beam parameters available in the different shot modes of Scarlet.

\begin{tabular}{lcc}
\hline Parameters & $400 \mathrm{TW}$ & $10 \mathrm{TW}$ \\
\hline Center wavelength & $815 \mathrm{~nm}$ & $810 \mathrm{~nm}$ \\
Repetition rate & $1 / \mathrm{min}$ & $10 \mathrm{~Hz}$ \\
Focused peak intensity & $10^{21}$ & $10^{19}$ \\
Pulse duration & $30 \mathrm{fs}$ \\
Pre-pulse contrast & $<10^{-8}: 1$ \\
Pointing stability & $25 \mu \mathrm{rad}$ \\
Shot energy stability & $< \pm 10 \%$ \\
Strehl ratio & $>0.9$ \\
\hline
\end{tabular}

pump as the compressor chamber, and can be taken down to $10^{-7}$ Torr levels with two turbomolecular pumps. The leaky mirror mount, target chamber off-axis parabola mount, and target positioning device have all been designed and built custom by the Ohio State team. They have the same precision parameters as previously mentioned for the compressor chamber mounts, and are also connected to the Scarlet network system for remote viewing and manipulation.

The off-axis parabola is a $175 \mathrm{~mm}$ diameter $F / 2$ focusing optic that brings Scarlet's 150 $m m$ diameter beam to an optimized $5 \mu m$ FWHM focus, shown in Fig. 4.7. The parabola is directly protected from target debris by a $4 \mu \mathrm{m}$ thick nitrocellulose pellicle similar to the one used between the compressor and target chambers.

Target alignment is achieved through alignment beams both side-on to the target and co-propagating with the short pulse beam. Reflective alignment techniques available 


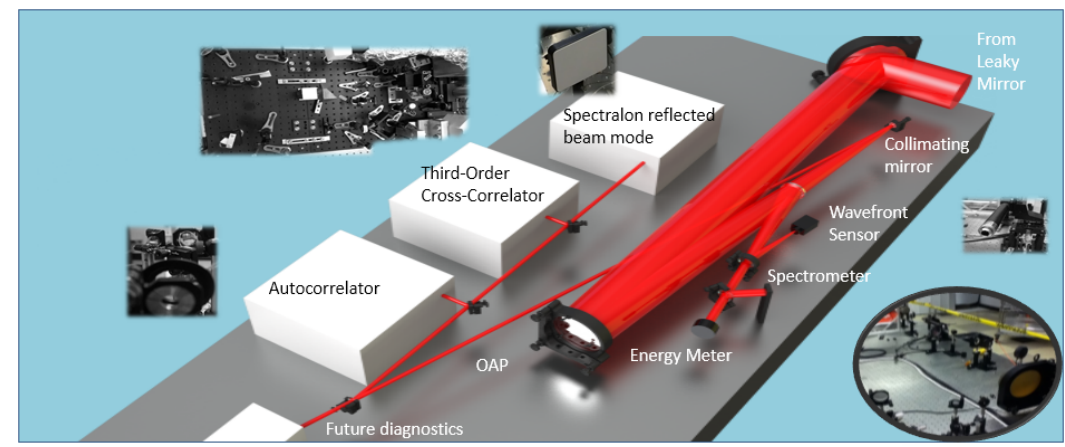

Figure 4.6: Schematic of the Scarlet laser diagnostics table. This setup includes an autocorrelator for pulse width measurements, cross-correlator for contrast measurement, Spectralon reflection for mode quality diagnosis, as well as an energy meter and spectrometer for standard pulse characterization.

include both scatter and specular light collection arms, as well as an in-vacuum objective lens and camera situated behind the target that can be used for alignment or focal spot characterization and then moved away for a shot.

A positioner based on confocal microscopy has been designed[75] that provides micronscale target positioning along the laser propagation direction. The confocal positioner utilizes a $532 \mathrm{~nm}$ alignment beam focused at normal incidence onto the target. This light is then recollected by the $10 \times$ plan-apochromatic focusing objective and imaged to a $10 \mu \mathrm{m}$ core multimode fiber that sends signal out of the target chamber to a variable gain photodiode. Final laser propagation direction alignment to optimize this signal gives repeatable, $\mu m$ scale precision for target regardless of surface morphology. Unlike conventional specular or scatter alignment diagnostics, this confocal positioner has been demonstrated on smooth or rough metal foils, reduced-mass targets with low surface area for reflectance, transparent thin film targets, and even targets with micron-scale structures on the front. This allows repeatable target alignment within the $10 \mu m$ Rayleigh range of Scarlet's $F / 2$ focus. The details of the device's operation will be discussed further in Chapter 9.

The Scarlet target chamber, shown in Fig. 4.8, currently uses two Highly-Ordered Pyrolytic Graphene (HOPG) spectrometers[76] to measure k- $\alpha$ and k- $\beta$ lines from copper or gold, as well as XUV cameras with spherically bent crystals to detect 68, 256, and $394 \mathrm{eV}$ radiation from the target. A Thomson parabola of a compact design[77] can distinguish between particle species and energies over a range of 1 to $35 \mathrm{MeV}$. Additional diagnostics include bubble detectors for neutron measurement, Micro-Channel Plates and scintillators for neutron and gamma flash measurement, as well as radiochromic film (RCF) and Columbia Resin 39 (CR39) particle detection materials. All of the digital diagnostics 


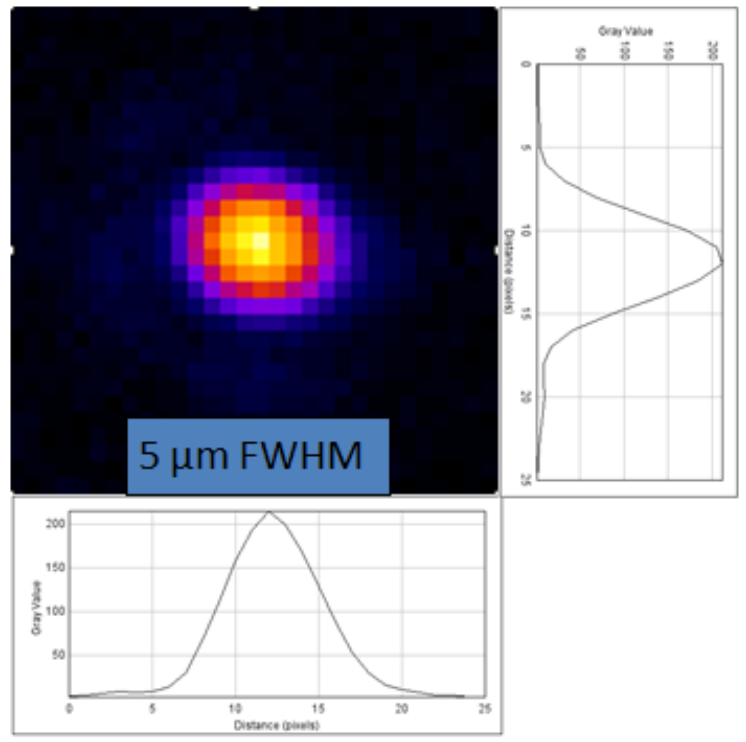

Figure 4.7: The Scarlet focal spot, as observed at a low energy level on the focal spot alignment camera. The size is $<5 \mu m$ FWHM.

have been wired into the Scarlet network to allow instant data collection and storage.

\subsection{Conclusion}

Like any state-of-the-art laser facility, Scarlet is constantly under improvement. At the time of this writing the new target chamber is being populated with beam steering optics as well as a number of new diagnostics. This sort of work, most of it entirely designed and implemented by students, ensures that Scarlet remains an important tool for High Energy Density Physics (HEDP) research. 


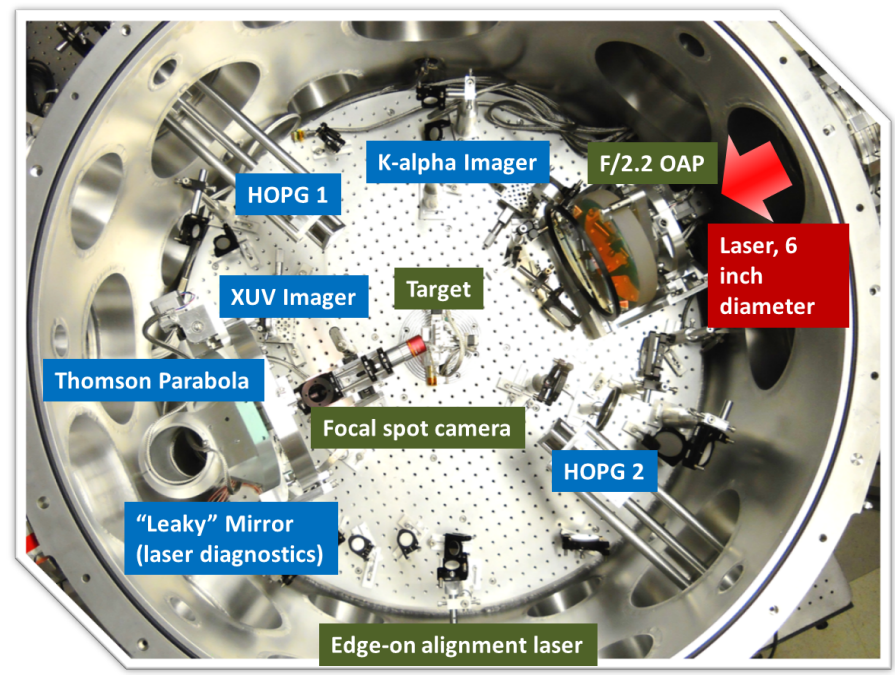

Figure 4.8: An image of the Scarlet experimental chamber during a previous experiment. Various alignment and target diagnostics can be seen. The laser is incident on the target from the upper right region of the picture. 


\section{Chapter 5 \\ Damage Testing of Intense Laser Components}

\subsection{Overview}

While studying ultra-intense laser-matter interaction, it is important not to forget the effects that lower intensity light has during the amplification process. Specifically, large efforts are committed to the study of the properties of materials up to and just over the damage threshold, or the laser fluence at which their morphology changes irrevocably. Such considerations are important for protecting expensive optics within a laser system but also because the fundamentals of laser damage are not yet fully understood-especially the differences between the $f s$ and $p s$ damage timescales. What follows is a damage testing study performed as part of the Scarlet $400 T W$ upgrade.

\subsection{Introduction}

Since chirped-pulse amplification (Chirped Pulse Amplification (CPA)) lasers[64] became the workhorse of modern high-intensity short-pulse laser experiments, further technological advances on such systems have proceeded rapidly. This progress is culminating in several $>10$ petawatt-scale laser development projects currently under way, each requiring high efficiency, broadband, damage-resistant pulse-compression gratings (PCG) of increasing size.[78] The gratings, particularly the final one in the pulse compressor of these large scale short pulse laser systems, are typically the least damage-resistant and most expensive optical elements in the laser chain. As a result, the maximum allowable fluence through the system as a whole is dictated directly by the size and robustness of the PCGs. Therefore, a better understanding of the damage mechanism and a more accurate estimate of the laser-damage threshold (LDT) of PCGs are both critically important for the economic feasibility and longevity of $P W$-class short-pulse laser systems.

It is well known that the damage mechanisms for femtosecond laser interaction differ 
from those for nanosecond or longer pulses.[79-84] Nanosecond pulses cause damage by material heating resulting in melting or ablation; this involves laser energy being absorbed by electrons which, on this timescale, stay in thermal equilibrium with the surrounding atomic lattice. In contrast, femtosecond pulses couple energy into the material by ionizing atoms (via multi-photon ionization initially, and then by avalanche ionization in the case of dielectrics) and accelerating the freed electrons (second step in dielectric, first step in metals) in the laser field. After gaining energy from the laser, these non-thermal electrons de-excite via $e-e$ collisions to a thermal bath which deposits its energy into the surrounding lattice via electron-phonon collisions. This dissipation of energy occurs over a very short length scale compared to that of longer pulses, and the result is highly-deterministic damage sites with relatively less morphological change to the surrounding area. This behavior has led to intense research in the areas of femtosecond etching and lithography.[85, 86] For reflection gratings, the damage mechanism is further complicated by surface morphology and any layered structure.[82]

Following some pioneering work on metal overcoat damage[81] and optimum grating design, $[82,83]$ much of the work on PCGs has focused on multilayer dielectric gratings, [8790] which function via interference from layers of material with alternating high and low indices of refraction. The dielectric material allows for a higher damage threshold than metal overcoated gratings, [91] but has been unsuitable for short-pulse lasers due to its narrow high-diffraction-efficiency bandwidth-this number has typically been $40 \mathrm{~nm}$ or less, [87, 88] although recent results are promising.[92] Nevertheless, these dielectric gratings can be difficult to manufacture on the scale necessary for high-power systems, and may suffer delamination due to internal stress buildup when exposed to vacuum.[87] As such, metalovercoated PCGs remain the standard for broadband, short-pulse (below $100 \mathrm{fs}$ ), highpower systems.

Another aspect of short-pulse laser damage involves the nonlinear index of refraction $n_{2}$. For air this parameter is small $\left(4 \times 10^{-23} \mathrm{~m}^{2} / W\right)$, but when coupled with intense, short-pulse light it can cause nonlinear-phase accumulation. This phase leads to breakup of the beam into multiple filaments that can then self-focus. The result is a spatially-varying fluence that may, in localized regions, exceed the expected fluence for a given laser energy and spot size. Compression and the subsequent propagation of short pulses must therefore be performed under vacuum, typically $<10^{-4}$ Torr. The vacuum system provides an ultraclean environment that is simultaneously thermally and atmospherically stable. For LDT determination with short pulses, the laser is typically focused to $\sim 10 \mu \mathrm{m}$ in diameter on the surface of a test optic. The pulses therefore may accumulate significant non-linear phase, even during propagation through air, which can cause an inaccurate measurement of LDT. For maximum operational relevance and accuracy, LDT of critical optical components for $P W$ scale lasers should be determined under vacuum. Prior to these experiments, no other 
published work existed for vacuum LDT determination of PCGs.

The following is a study of the damage threshold of several types of metal-on-dielectric PCGs in vacuum. Au-coated gratings are used almost exclusively on all high-power systems at near-infrared (IR) wavelengths with pulse widths shorter than $100 \mathrm{fs}$. This is due to their high reflectivity and longevity when compared to other metallic coatings and to their broad bandwidth and resilience to vacuum environments when compared to multilayer dielectric gratings (as mentioned previously). Despite their ubiquity, Au-coated gratings do have some distinct disadvantages. Firstly, known short pulse LDT values are around 200 $m \mathrm{~J} / \mathrm{cm}^{2}$, whereas multilayer dielectric gratings have a typical LDT between $1-2 \mathrm{~J} / \mathrm{cm}^{2}$.[88] Another main problem is grating blackening under prolonged vacuum operation.[93-95] Vacuum contamination on optical surfaces is also known to lower the damage threshold substantially.[96] For these reasons, short pulse fluence (beam normal) on the PCGs are kept strictly below $100 \mathrm{~mJ} / \mathrm{cm}^{2}$. Additionally, expensive and cumbersome RF plasma cleaning systems have to be installed to keep PCGs operational for a prolonged period of time.[93]

\subsection{Grating fabrication}

There are two main methods for manufacturing reflective diffraction gratings: mechanical ruling and holographic surface relief. Mechanically-ruled gratings have been in use for well over a century, and much progress has been made toward increasing the maximum ruling density and precision as well as improving the uniformity with which grating surfaces can be etched.[97] Although high groove quality is achievable with modern advances, this mechanical etching method can still produce gratings of insufficient quality for some applications.[97]

An alternative to mechanically-ruled gratings utilizes the fringe pattern arising from the interference of two monochromatic collimated beams of ultraviolet (UV) light. Here the substrate (again, often fused silica) is coated with a layer of photoresistive material, which has the property that its chemical bonds are broken upon irradiation with UV light. After illumination, a chemical developer etches away the broken bonds, forming the desired grating structure. A metallic film can then be applied to the photoresist layer. In theory, this method can produce ruling patterns devoid of any groove placement errors, potentially at a faster rate than mechanical ruling.[97] However, it requires precise and homogeneous light exposure. Furthermore, the photoresist layer may modify the damage properties of the grating.

Plymouth Grating Laboratory has developed a serialized grating fabrication technique that performs energetic-sputter coating of metals. This technique utilizes improvements on the Nanoruler metrology system,[71] which uses scanning beam interference lithography 
as described above and is equipped to create grating patterns on optics larger than 300 $\mathrm{mm}$. This precise ruling system, along with their newly-developed energetic-sputtering technique for smoother overcoats, allows for more uniform final grating structures that improve diffraction efficiency, as shown in Fig. 5.1. This technique was used to create the gratings used in the Scarlet laser system.
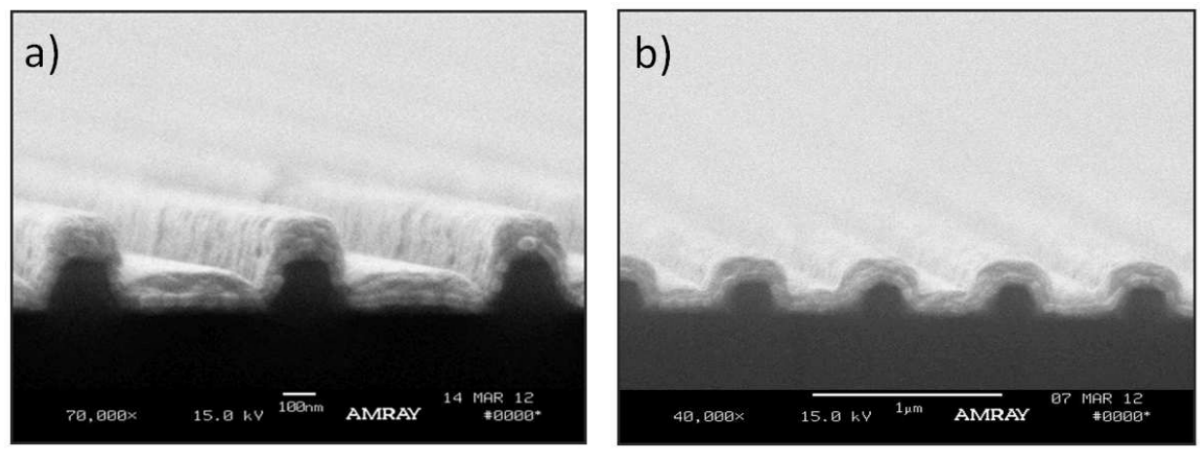

Figure 5.1: (a) Scanning electron microscope (SEM) image of the conventional sputtercoated grating exhibiting a non-conformal groove structure that will decrease the diffraction efficiency. (b) SEM image showing the energetic sputter-coated grating with a more uniform groove structure, from Plymouth Grating Laboratory.

The gratings tested here had a groove density of 1480 lines $/ \mathrm{mm}$ and were all made on a $50 \mathrm{~mm}$ diameter fused-silica substrate by Plymouth Grating Laboratory. All gratings were tested with p-polarized light at $46^{\circ}$ angle of incidence. A bare (no protective dielectric overcoat) Au-coated fused-silica mirror (which was coated simultaneously along with the gratings) was tested as a control sample.

\subsection{Experimental setup}

The experiment was conducted using the front-end of the Scarlet laser. As was mentioned in Chapter 4, the front-end consists of a Ti:sapphire system that utilizes a nine pass amplifier situated between a glass stretcher and a prism compressor. This generates $800 \mu J$ pulses at a repetition rate of $1 \mathrm{kHz}$. The beam has a $55 \mathrm{~nm}$ bandwidth centered on $800 \mathrm{~nm}$. The distance between the compressor prisms can be manipulated to vary the pulse width from 25 to $200 \mathrm{fs}$. Single-shot operation was achieved by manipulating the timing of two Pockels cell triggers in the front end amplifier.

The front-end beam was sent to a vacuum chamber as shown in Fig. 5.2(a), where samples were tested at a pressure of around 0.1 Torr. A F/30 (300 mm) achromatic lens on a translation stage focused the beam onto the sample at an angle of $46^{\circ}$. For 

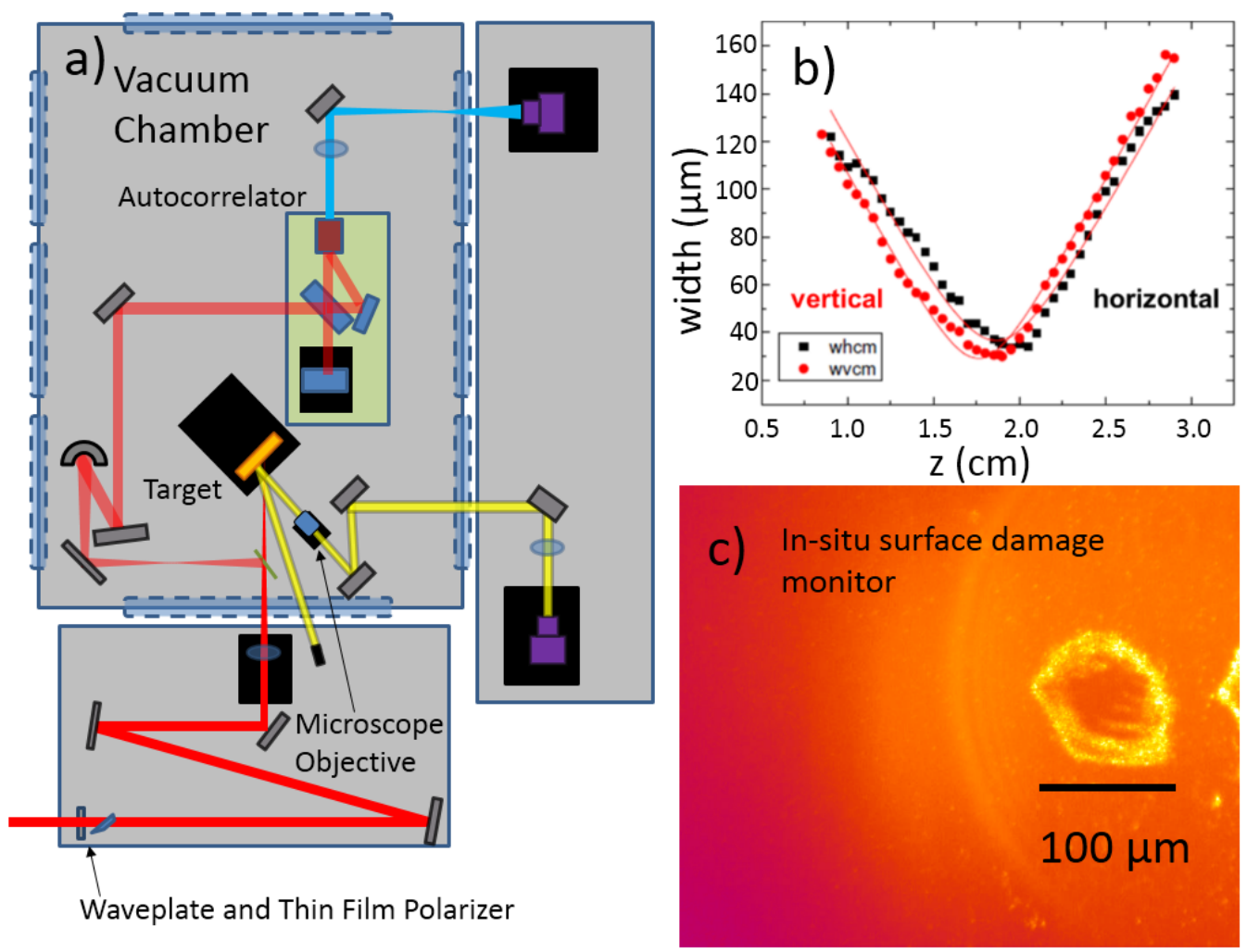

Figure 5.2: (a) Schematic of vacuum chamber for damage testing. Of note are the autocorrelator to measure pulse width and the $10 \times$ microscope objective for in-situ damage verification. (b) Focal spot evolution and respective fit showing $M^{2}<1.2$. (c) Example in-situ damage image in false color.

the data presented here, the fluence on target was varied by a zero-order half-waveplate and a broadband beamsplitting thin-film polarizer. When a greater range of fluences was desired, the focusing lens could be shifted along the laser axis to vary the fluence while keeping the pulse energy constant (used for LDT testing of samples not discussed here). This required detailed characterization of the beam waist, the data for which is shown in Fig. 5.2(b), showing near-perfect Gaussian beam characteristics. The target was positioned on a jig consisting of two vacuum picomotor-controlled translation stages, allowing for horizontal and vertical movement under vacuum. The jig maintained the angle of incidence within $0.5^{\circ}$ throughout its full range of motion. Damage was observed using an in-situ, $10 \times$, infinity-corrected, long working distance, plan-apochromatic microscope objective (Mitutoyo) with a $3.5 \mu \mathrm{m}$ depth of focus over a $0.5 \mathrm{~mm}$ field of view. The image was relayed onto a 16-bit camera (Basler A641f) outside vacuum using a $200 \mathrm{~mm}$ focal length achromatic lens. Damage was defined to be a visible change in the surface morphology or reflectivity visible through this in-situ microscope, an example image from which is shown in 
Fig. 5.2(c). Damages were subsequently documented with a separate microscope with up to $400 \times$ magnification. The pulse width on target, which was set by changing the separation of the prisms in the front-end compressor, was measured using an autocorrelator within the vacuum chamber. The pulse widths used were 30, 100, and $200 \mathrm{fs}$. Single-shot pulse energy was measured with a small-percentage pickoff optic sending light to a calibrated photodiode.

\subsection{Results}

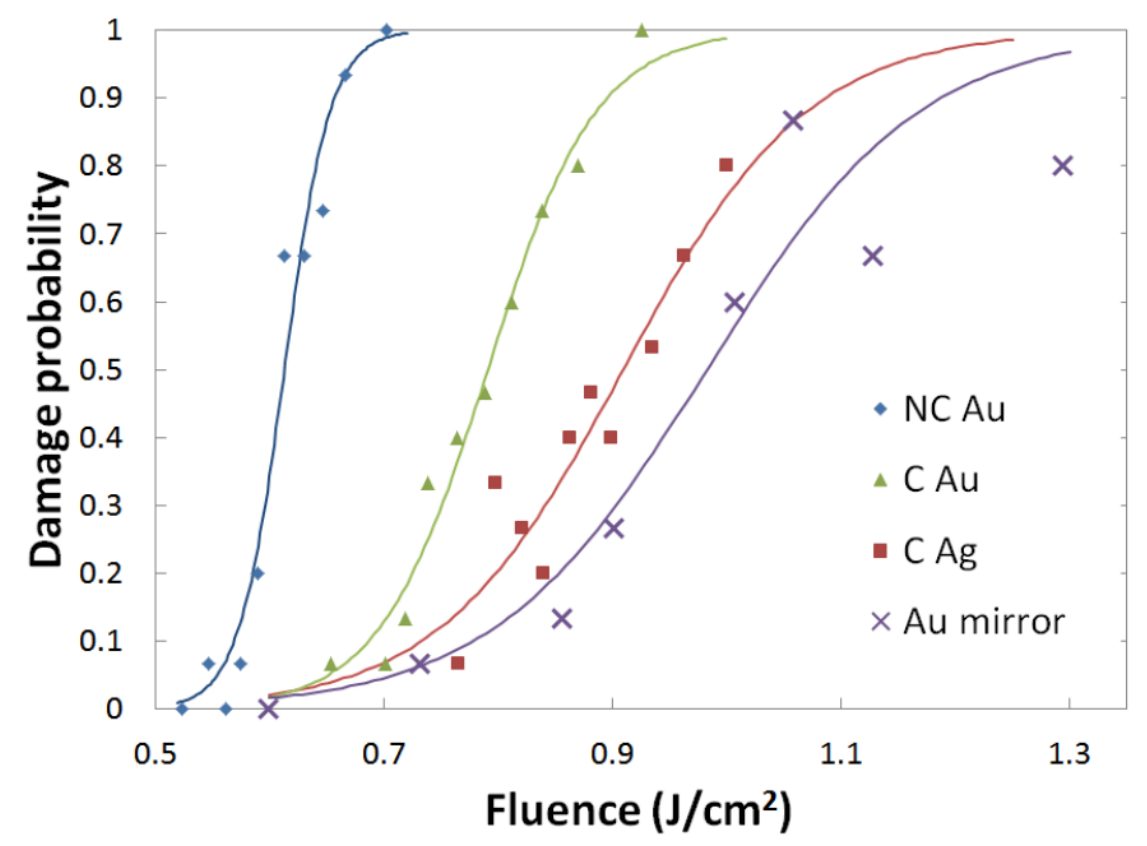

Figure 5.3: Damage threshold data with curve fits for non-conformal (NC) and conformal (C) samples irradiated with $30 \mathrm{fs}$ pulses. For the Au-coated gratings, the conformal coating has a higher damage threshold than the non-conformal coating. Each data point represents 15 shots with a fluence error of $\pm 0.02 \mathrm{~J} / \mathrm{cm}^{2}$.

Figure 5.3 shows the single-shot damage probability with respect to fluence in $\mathrm{mJ} / \mathrm{cm}^{2}$ for the $\mathrm{Au}$ and $\mathrm{Ag}$ conformal gratings and the non-conformal $\mathrm{Au}$ grating, as well as a bare-Au control sample. Though samples were tested at $46^{\circ}$, all fluences reported here have been converted to the beam-normal value. LDT is expressed in terms of the peak fluence for a Gaussian beam profile, $\Phi_{0}=\frac{2 E}{\pi w_{0}^{2}}$, where $E$ is the energy in the pulse, and $w_{0}$ is the Gaussian waist radius.[98] The damage probability in the femtosecond regime is not linear with fluence, [99] and so the curve fits here are generated from sigmoid functions 


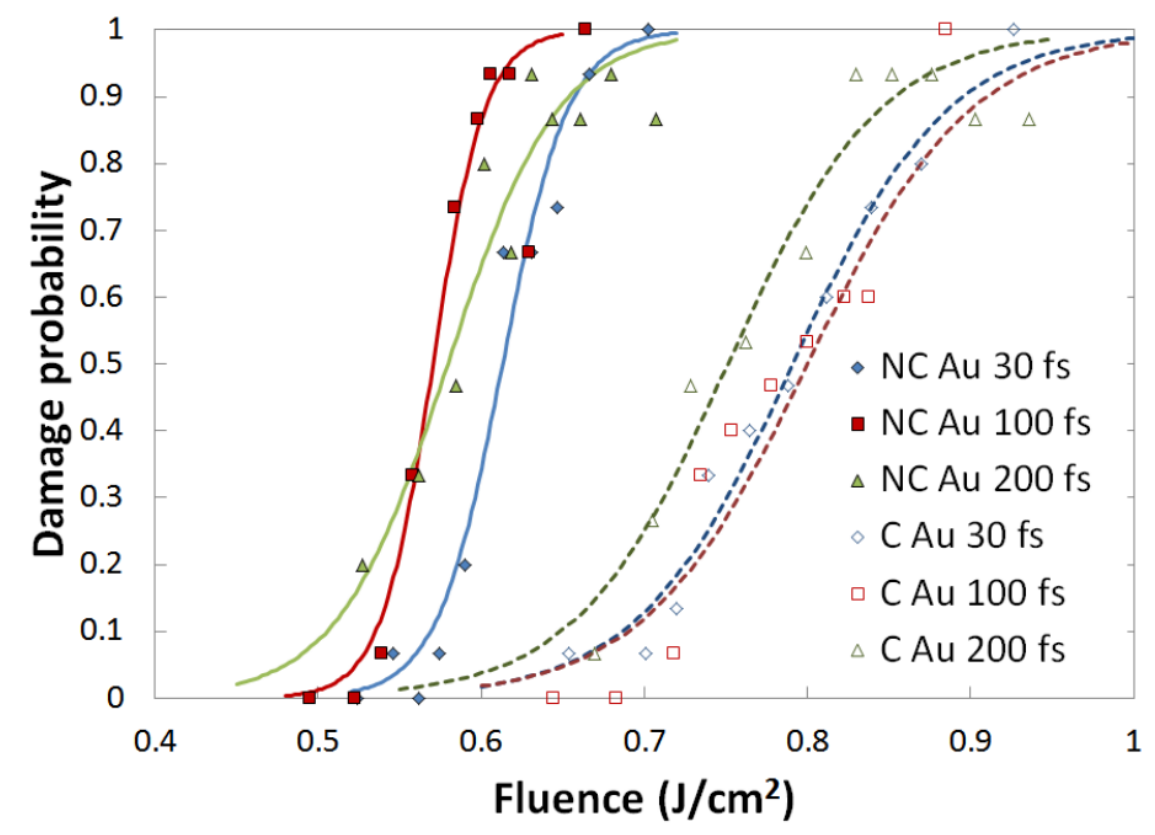

Figure 5.4: Pulse width dependence of damage threshold by sample. While the conformal (C) coating clearly has a higher damage threshold than the non-conformal (NC) coating, there is no clear pulse width dependence of the damage threshold observed for either grating type. Each data point represents 15 shots with a fluence error of $\pm 0.02 \mathrm{~J} / \mathrm{cm}^{2}$.

constrained to asymptote at 0 and 100 percent damage probabilities. Data was collected by observing damages at specific irradiation fluence on an area of the sample, providing a percentage of shots at a fluence level that resulted in visible damage. Between 10 and 20 shots were sampled for damage probability determination at each fluence. After irradiation the sample was translated by $100 \mu m$ to eliminate multi-shot effects[100-102] and to ensure that each exposure was conducted on a debris-free surface. Figure 5.4 shows the dependence of pulse width on the damage threshold. All curves exhibit a similar shape with limited variations in the damage threshold level. These results obtained with current gratings show a somewhat higher LDT than previous measured values for Au-coated gratings.[81] Neauport et al.[89] tested the LDT for Au-on-photoresist gratings with 1740 lines $/ \mathrm{mm}$ at $72.5^{\circ}$ angle of incidence with $500 \mathrm{fs}$ pulses and reported $0.67 \mathrm{~J} / \mathrm{cm}^{2}$. This corresponds to $0.28 \mathrm{~J} / \mathrm{cm}^{2}$ after correcting for the incidence angle, lower than the LDT of gratings tested here. For comparison with dielectric and metal dielectric gratings tested by Canova et al. [88], the LDT after correcting for their incidence angle is $\sim 0.67 \mathrm{~J} / \mathrm{cm}^{2}$.

The damage threshold data for the samples tested is summarized in Table 5.1; these numbers represent the fluence level at which zero percent of shots resulted in damage. Safe operation is possible on the conformal Au grating at a fluence of $200 \mathrm{~mJ} / \mathrm{cm}^{2}$, while maintaining a $2 \times$ safety factor. To determine the long-term parameters of these gratings, 
Table 5.1: Single-shot damage thresholds ( $0 \%$ damage probability) in $\mathrm{mJ} / \mathrm{cm}^{2}$ for three gratings tested at multiple pulse widths.

\begin{tabular}{lccc}
\hline & $30 f s$ & $100 f s$ & $200 f s$ \\
\hline Non-conformal $\mathrm{Au}$ & 560 & 520 & 460 \\
Conformal $\mathrm{Au}$ & 660 & 670 & 600 \\
Conformal $\mathrm{Ag}$ & 730 & 600 & 770 \\
\hline
\end{tabular}

multiple shot (1000 shots per test point) LDT measurements were also performed, which resulted in $\sim 20 \%$ lower LDT on all gratings tested here. Although published data on multiple fs shot LDT measurements on metals is scarce,[103] based on theoretical work by Wang et al.[104] and works on dielectrics, [105, 106] multiple shot threshold is predicted to be lower than single-shot due to heat buildup in the metal films from successive shots.

No LDT testing on the PCG samples were performed in air. However, to test how short pulse LDT is affected by vacuum, other PCG samples and bare metal mirrors have been tested previously in air. Compared to the vacuum values, atmospheric threshold was always found to be lower, typically by $30-40 \%$. This is attributed to the generally uncontrolled nature of atmospheric experiments on surfaces due to exposure to dust and other airborne particles, humidity, and also non-linear phase accumulation at higher fluences.

A pair of 1480 lines $/ \mathrm{mm}$ conformal Au gratings of the type studied here have been used to compress full energy (24 $J$ uncompressed, $43 \mathrm{~nm}$ bandwidth, $130 \mathrm{~mm}$ beam diameter) pulses to $30 \mathrm{fs}$. The all-aluminum-construction compressor chamber in Scarlet is kept below $2 \times 10^{-7}$ Torr with oil-free turbomolecular pumps backed by a dry screw pump. The highest beam normal average fluence at short pulse intensity experienced by the smaller grating at the final pass is $130 \mathrm{~mJ} / \mathrm{cm}^{2}$. The beam profile incident on these gratings resembles a "flat-top" profile, and therefore the peak fluence is closer to the average fluence than in the case of a Gaussian beam. The grating sizes are $340 \mathrm{~mm}(\mathrm{w}) \times 420 \mathrm{~mm}(\mathrm{~h})$ and $560 \mathrm{~mm}$ $(\mathrm{w}) \times 420 \mathrm{~mm}(\mathrm{~h})$. Analysis of both gratings at a wavelength of $805 \mathrm{~nm}$ and $46^{\circ}$ angle of incidence shows an average diffraction efficiency of $\sim 93 \%$. An autocorrelation trace lineout taken near the installation time of these gratings is shown in Fig. 5.5. The compressor efficiency is $65 \%$ and has remained unchanged for several years.

\subsection{Discussion}

Damage morphology was observed to depend on the surface structure of the tested grating. For the Au gratings, the in-situ camera revealed that damages had a similar morphology and change in reflectivity when compared to those observed on the bare-Au mirror. In all of these cases, the observed reflectivity change was due solely to an ablation of the Au layer and not a result of direct damage to the groove pattern on the substrate below; this can 


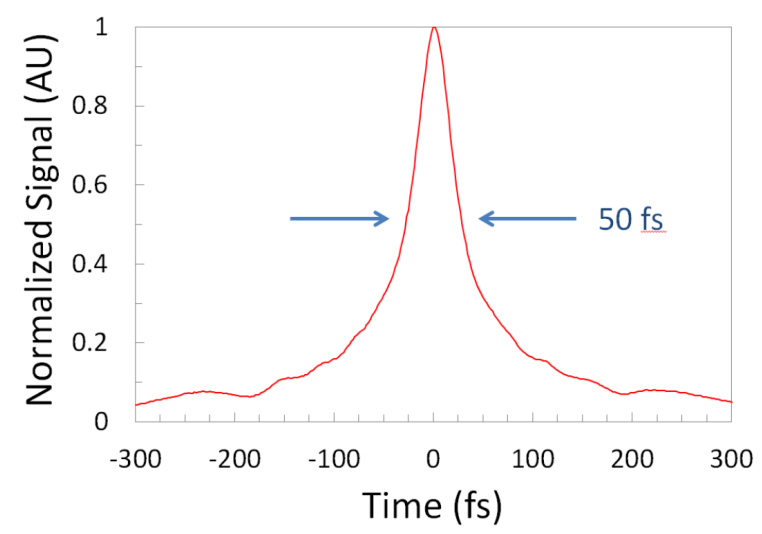

Figure 5.5: Autocorrelation trace of a $50 \mathrm{fs}$ pulse after compression by conformal $\mathrm{Au}$ gratings at the Scarlet laser facility. This initial measurement has since been surpassed by further grating optimization, allowing $30 \mathrm{fs}$ pulse compression to be achieved currently.

be seen in Fig. 5.6, which shows a SEM image of a conformal Au damage site from a 950 $\mathrm{mJ} / \mathrm{cm}^{2}$ pulse. Therefore, an advantage of this type of grating is the possibility of recoating at a fraction of the cost of replacing the entire optic. Since these measurements, full-size gratings have been fully-stripped of their coatings and then successfully recoated.

Numerical simulations were conducted in order to assess the effects of surface morphology variations of the Au coating on grating diffraction efficiency and laser damage threshold. In particular, careful attention was paid to detect any field enhancement effects caused by non-conformal groove features, which were not present in the near-sinusoidal gratings reliefs discussed by Boyd et al.[82] The simulations were performed using the commercial finite elements method package Comsol Multiphysics 4.3a. A 2D Frequency Domain study in the Radio Frequency module of the software was used. The simulation domain was terminated by periodic boundary conditions, with the period set to $\mathrm{P}=675$ $\mathrm{nm}$ in the direction along the grating surface, and a periodic input/output port in the direction normal to the surface. The same port is used for injecting the incident wave and for collecting the reflected waves. The periodic ports can accommodate an incoming plane wave at any angle of incidence with any preset number of diffractive orders; it does so by being transparent to the radiation along those modes and tracking the power flow among them. The incoming monochromatic plane wave with wavelength $800 \mathrm{~nm}$ is incident at an angle $\theta=46^{\circ}$ with respect to grating normal. Only the zeroth (specular) and the negative first diffractive orders are propagating in reflection for the design wavelength of $\lambda=800$ $n m$. These propagating diffractive orders are accounted for by the periodic port boundary condition. All higher diffractive orders are evanescent in the simulation and do not reach the input/output port, which is placed at a distance of $\Delta z=3 \lambda$ from the grating.

$\mathrm{Au}$ is modeled using the complex permittivity interpolated from tables generated by 


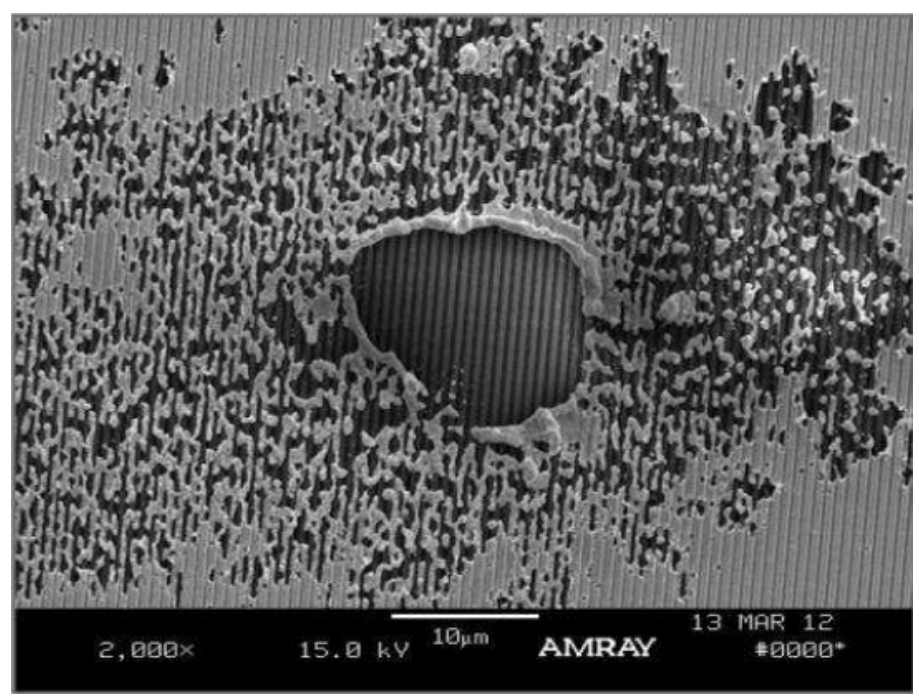

Figure 5.6: SEM image showing intact fused silica substrate at a damage site on the conformal Au grating, produced from a $950 \mathrm{~mJ} / \mathrm{cm}^{2}$ pulse.

Palik.[107] The surface shape of the Au layer is traced out by using second order (quadratic) Bezier curves so that the outline is consistent with SEM images of the groove profile (Fig 5.1). Three different curves corresponding to different levels of conformal coating are simulated. Less conformal coatings form a more pronounced Au lump between the grating ridges. Reflectance into the zeroth and negative first diffractive orders is obtained from the scattering parameter output of the code. The results of the simulation are shown in Fig. 5.7, where three conformal $\mathrm{Au}$ coverages are shown in (a), ranging from least conformal $(\mathrm{C} 1)$ to most conformal (C3). Note that the least conformal grating showed the strongest field enhancement at the Au surface. This translates into the highest Joule heating of the grating. Table 5.2 details the absorptivity $\mathrm{A}$, grating efficiency $R_{-1}$ (i.e. scattering into the desirable negative first diffractive order), and parasitic (specular) reflectivity $R_{0}$. These values were calculated in the simulation and also measured on the actual gratings that corresponded to the simulated curvatures. The inferred absorption from the reflectivity measurements is also shown in the table for comparison, as well as the corresponding measured LDT.

In measurement, the highest damage threshold was obtained for the conformal grating, which had a negative first order diffraction efficiency of $92 \%$. The slightly non-conformal grating, which exhibited a significantly lower damage threshold, had $84 \%$ efficiency. These gratings had reflection efficiencies of $0.5 \%$ and $4 \%$, respectively. These measurements were performed using broadband femtosecond pulses with a spectrum centered on $800 \mathrm{~nm}$, with root mean square (RMS) error $<0.5 \%$ for all measurements. Any loss due to scattering was not measured, and assumed to be minimal due to the smooth surface of the Au coating. It is apparent that the model somewhat underestimates the diffraction efficiency of the 


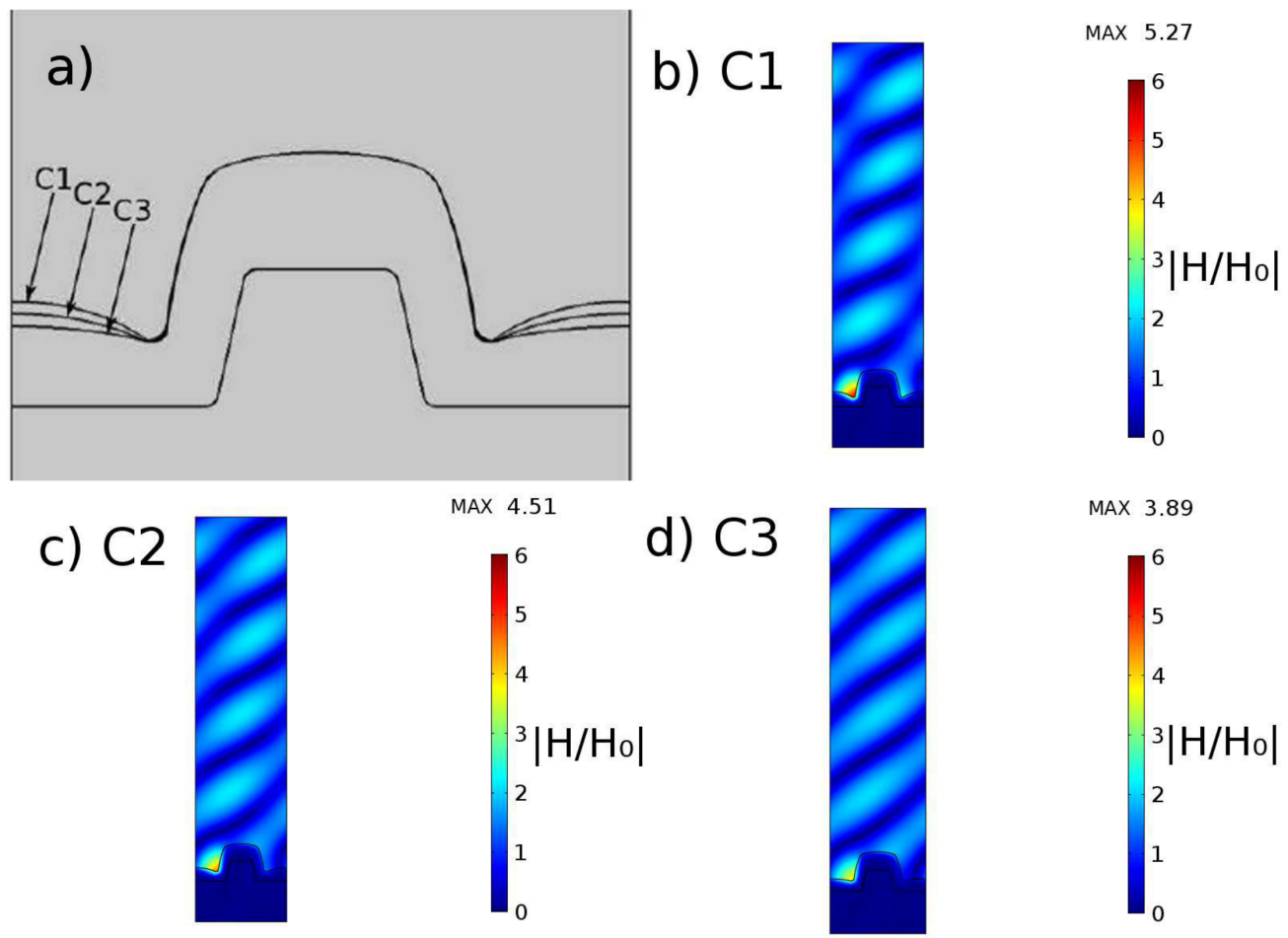

Figure 5.7: (a) Shapes of the three different Au surfaces corresponding to differentlyconforming $\mathrm{Au}$ layers. (b)-(d) Color-coded magnetic field enhancement $\left|H / H_{0}\right|$ for the gratings C1-C3, respectively, centered around one diffraction ridge (visible at the bottom of each image). Here $H_{0}$ is the magnetic field of the p-polarized light wave with $\lambda=800 \mathrm{~nm}$, incident at an angle $\theta=46^{\circ}$. The max value of $\left|H / H_{0}\right|$ is indicated above each color bar, and decreases for the more conformal curvatures. Other wavelengths within the experimental range were also simulated, with similar field enhancement results to those shown here.

conformal and non-conformal gratings when compared with model gratings C3 and C2, respectively. This is most likely because of two reasons: the uncertainty of curve-fitting of grating surface profiles, and the fact that diffraction efficiency as a function of wavelength is not constant. However, the model does qualitatively capture the physical picture, i.e. that the non-conformal grating with higher absorbance and lower diffraction efficiency undergoes higher Joule heating via energetic electrons trapped by field enhancement.

Additionally, the increased damage threshold of the Ag-coated grating $\left(\sim 600 \mathrm{~mJ} / \mathrm{cm}^{2}\right)$ is of note. Originally the experiment was intended to test the effect of conformal $\mathrm{Au}$ coatings, but promising initial results prompted a test of the conformal Ag coating as well. Traditionally, Ag coatings are not widely used for large gratings due to a susceptibility to tarnish over time. However, the observed intact substrate upon damage of the conformal coating type (seen in the $\mathrm{Ag}$ coating as well as $\mathrm{Au}$ ) suggests recoating as a cheap alternative to replacement of the optic, potentially making Ag-coated conformal gratings a viable 
Table 5.2: Measurements and simulation calculations of the absorptivity A, negative first diffraction order reflectivity $R_{-1}$, and specular reflectivity $R_{0}$ for three different grating profiles (with C3 being the most conformal), as well as measured LDT values for the relevant profiles

\begin{tabular}{lcccccccc}
\hline & \multicolumn{3}{c}{ Calculated } & & \multicolumn{5}{c}{ Measured } \\
\cline { 2 - 4 } \cline { 7 - 9 } Grating model & $R_{-1}$ & $R_{0}$ & $A$ & & $R_{-1}$ & $R_{0}$ & $A$ (inferred) & LDT $\left(\mathrm{mJ} / \mathrm{cm}^{2}\right)$ \\
\hline C1 & $60.3 \%$ & $28.1 \%$ & $11.6 \%$ & & & & & 560 \\
C2 & $78.4 \%$ & $13 \%$ & $8.6 \%$ & & $84 \%$ & $4 \%$ & $12 \%$ & 660 \\
C3 & $88.9 \%$ & $4.5 \%$ & $6.6 \%$ & & $92 \%$ & $0.5 \%$ & $7.5 \%$ & \\
\hline
\end{tabular}

alternative to the current standard Au type. More study is required to decrease the cost of recoating further, and to preserve the reflective qualities of $\mathrm{Ag}$ for a longer duration. One possibility is adding a thin dielectric coating on top of the Ag coat. This procedure has increased the damage threshold at longer pulse lengths by a factor of two,[108] and on $\mathrm{Ag}$ may have the added benefit of preventing tarnish.

\subsection{Conclusion}

This Chapter reports on damage threshold results for various gratings with metal reflective coatings. The data shows no clear pulse width dependence on LDT for pulses between 30-200 $\mathrm{fs}$. Simulations show that the decreased diffraction efficiency and damage threshold of the non-conformal grating is due to field enhancement resulting from convex surface morphology of the groove coating valleys, which the conformal coating method eliminates. The conformal $\mathrm{Au}$ grating on fused silica has the advantage of possible restoration of its diffraction efficiency fully by stripping the damaged Au layer and recoating, even after the metal coating sustains significant damage. Due to their higher LDT, compressors consisting of conformal-groove gratings should be able to safely operate at fluences of $200 \mathrm{~mJ} / \mathrm{cm}^{2}$ (as opposed to the current typical value of $100 \mathrm{~mJ} / \mathrm{cm}^{2}$ ), reducing the beam aperture and all post-compressor optical component sizes, thereby allowing significant cost savings in design, construction, and maintenance of $P W$-class laser systems. 


\section{Chapter 6 \\ Liquid Crystals as Scientific INSTRUMENTS FOR INTENSE LASER APPLICATIONS}

\subsection{Overview}

Ubiquitous in modern displays and screen technology, liquid crystals have long been studied as materials with unique and useful properties. Their most familiar applications rely on modulating the way light passes through the liquid crystal based on reorientation of the constituent molecules via an applied voltage. Placing a layer of liquid crystal between crossed polarizers and then manipulating the orientation of the liquid crystal molecules with a voltage results in an optical switch which can then be implemented in the pixels of a display. While this property has not yet been exploited for liquid crystals in high intensity laser experiments, it has some potential applications which will be discussed. Here their primary utility comes from their ability to form thin, variable thickness freely suspended films under high vacuum. What follows is a brief introduction to the relevant chemistry and physics of liquid crystals pertaining to forming such films.

\subsection{Brief History}

The first study of a liquid crystal substance dates back to Austria in 1888, when botanist Friedrich Reinitzer observed what appeared to be two distinct melting points in a substance that was related to cholesterol. As the substance was heated it would turn first from solid to a cloudy liquid, and at a somewhat higher temperature the liquid would then become clear. Different color effects would also occur in this substance as it was cooled through these two transitions. At the time it was suspected that these extra phase transitions involved different levels of crystallinity within the substance. Not long after, physicist Otto Lehman watched these phase transitions under a polarizing microscope, further revealing the varying 
structure. The clearly crystalline nature of the material led Lehman to call this and other related substances "soft crystals", later known as liquid crystals.

Research on liquid crystals continued through the 20th century with the classification of the different types of liquid crystal phases, some of which will be detailed here. Though much of the intense liquid crystal study of the 1970s and beyond focused on the synthesis of these materials for fundamental research on their structure-property relationships, the most visible aspect of liquid crystal research lies in their use in modern displays. As will be discussed, the structure of liquid crystals can have useful optical effects, including polarization rotation that is switchable due to a reorientation of liquid crystal molecules via an applied voltage.

Before delving into these conventional liquid crystal applications as well as their utility as intense laser-matter targets, it is worth discussing the chemistry that results from the unique properties of these molecules and their various material phases.

\subsection{Relevant Chemistry}

Liquid crystals are distinguished from conventional matter by the appearance of additional phase transitions between solid and liquid. These extra "middle" phases are called mesophases and have different levels of molecular ordering both in orientation and position. In the case of thermotropic liquid crystals these phase transitions occur due to temperature changes, but they can also be induced via solvent/solute concentration changes in what are called lyotropic liquid crystals.

The most basic liquid crystals are comprised entirely of hydrocarbons, although fluorine, oxygen, and nitrogen are not uncommon additions,[109] and molecules containing metals from iron to mercury to gold[110] can be synthesized as well. Though the exact molecular composition can have a profound effect on the behavior of a liquid crystal, especially if some local polarity is induced as a result, it is the structural characteristics derived from these constituents that have the most visible impact.

Across the hundreds of chemical compounds that classify as liquid crystals, a few common structural features exist. A necessary piece is some sort of rigid, planar structure in the center, often a number of aromatic rings (e.g. phenyl or pyrimidine groups) or a ligand structure, possibly with other connecting elements in between. It is this central structure that provides stability to the molecule as a whole and is the section that will be rigidly ordered within a liquid crystal phase. This core structure can have effects on the melting temperatures, dielectric polarizability, optical anisotropy, and vapor pressure, among other relevant physical parameters.[109]

There are in general two patterns that any lateral substituents from the central structure can exhibit. Often there are simply two tail structures, one on either side of the central 


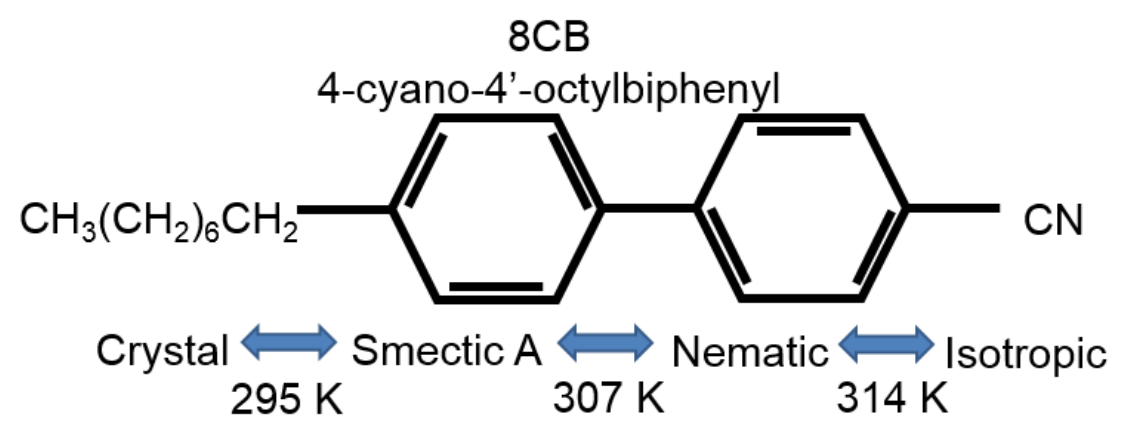

Figure 6.1: Chemical formula of the liquid crystal $8 \mathrm{CB}$, along with its mesophases and their transition temperatures.

region; these liquid crystal molecules are given the name calamitic, or rod-like. Alternatively there can be a greater number of tail structures extending radially from the center, forming a discotic liquid crystal. The phases exhibited by these different molecular shapes can vary, and will be discussed in detail below.

For calamitic liquid crystals, often a long-chain alkyl tail occupies one or both ends of the molecule, giving it lipid-like chemical properties. This tail can have interstitial links of other elements, branching structures, and lateral side-chains, all of which change the nature and range of the phases exhibited by the liquid crystal. As an example: intertwining of the longer chains from adjacent molecules tends to stabilize a more ordered crystal phase, increasing its temperature range. Polar terminal groups tend to twist one molecule with respect to its neighbor, which promotes other phases. The exact nature of these three formational structures-the central structure and two tails-and the way they are connected to each other determines a number of details about the behavior of the liquid crystal including which mesophases it exhibits, at what temperature the phase transitions occur, and physical properties like surface tension, fluid flow velocity, and vapor pressure.[109]

The chemical formula of the main liquid crystal used in this work, 4-cyano-4'octylbiphenyl $(8 \mathrm{CB})$, is shown in Fig.6.1. This is a calamitic molecule, with a central biphenyl structure providing rigidity. Here there is one long-chain alkyl tail which tends to intertwine and stabilize certain phases, while the other end of the molecule terminates in a short cyano group influencing the preferred molecular orientation. $8 \mathrm{CB}$ has both a nematic phase and smectic phase, to be described below.

What follows is a brief summary of the most common mesophases, with an emphasis on their properties that are most relevant when forming thin films. 

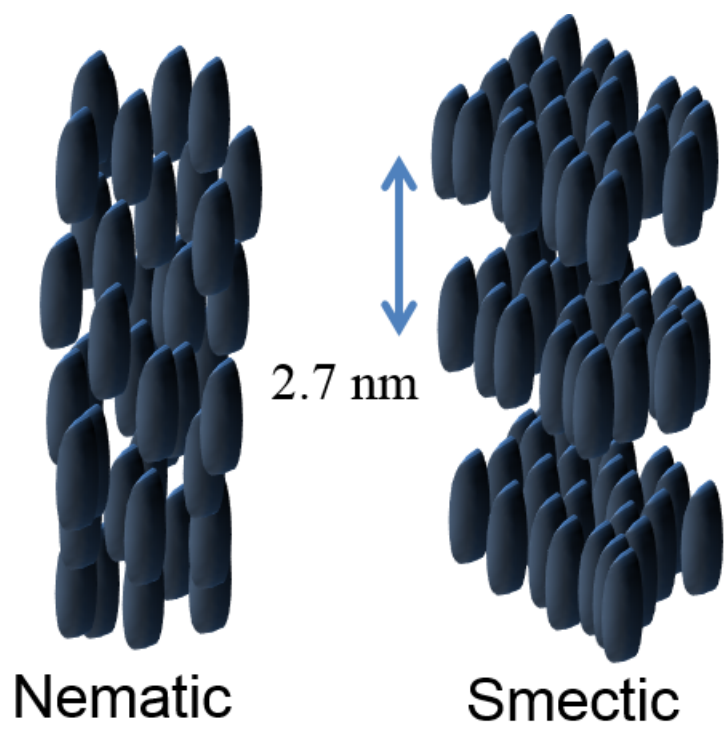

Figure 6.2: Cartoon of two common liquid crystal mesophases: nematic, which exhibits only orientational order, and smectic, which has orientational and positional order.

\subsection{Mesophases}

Mesophases are most easily distinguished by their different levels of positional and orientational order. To discuss this the concept of the director is typically used: the director is defined as a dimensionless unit vector that points along the long axis of a single liquid crystal molecule. If all the molecules in an area are aligned in one direction, the local director can be used to describe that direction. For reference, this director can also be used to define the crystal axis in mesophases which exhibit enough order to have one. A cartoon of two mesophases to be discussed here is shown in Fig. 6.2.

A typical characterization of the orientational order of a liquid crystal mesophase is given by the spatial and temporal average of the second order Legendre polynomial:

$$
S=\left\langle P_{2} \cos (\theta)\right\rangle=\left\langle 3 / 2 \cos ^{2}(\theta)-1 / 2\right\rangle .
$$

Here $\theta$ represents the angle between the primary axis of a molecule (the longest one in the case of the calamitic crystals under consideration here) and the local director for the surrounding liquid crystal volume unit. As mentioned the angle brackets denote both temporal and spatial averages. $S$ is a convenient measure of liquid crystal order since for a perfectly disordered system it averages to 0 , and for a perfectly ordered system the average evaluates to 1 . Most liquid crystals have order parameters between 0.3 and 0.8 , with the value in general decreasing with temperature. This orientational order parameter can be measured via a number of techniques including birefringence, nuclear magnetic resonance, 
diamagnetism, and Raman scattering.[109]

For $8 \mathrm{CB}$, the nematic phase has an order parameter that increases with decreasing temperature from 0.3 and 0.5.[111] Once $8 \mathrm{CB}$ enters the more ordered smectic phase, the order parameter shifts from 0.5 to 0.7 across the temperature range of that phase.[112]

If an additional measure of orientational order is desired, higher order even Legendre polynomials may be used. These are more difficult to measure, although $P_{4}$ can be done so with sensitive measurements like neutron scattering or electron spin resonance.[109] Since the director is defined equivalently for the $0^{\circ}$ or $180^{\circ}$ direction, all odd Legendre polynomials average to 0 .

Some phases of liquid crystals, namely the smectic phase to be introduced shortly, have positional order in addition to orientational order. In this case the density of liquid crystal $\rho$ as a function of depth $z$ is used:

$$
\rho(z)=\rho_{0}\left(1+\psi \cos \left(\frac{2 \pi z}{d}\right)\right)
$$

where $\rho_{0}$ is the average density, $d$ is the average distance between layers, and $\psi$ is the complex positional order parameter.

The phase of $\psi$ denotes where along the $z$ axis layers lie with respect to a fixed coordinate system. The amplitude of $\psi$ is typically much less than 1 , and decreases with temperature. In this way a smectic phase may not form perfect layers as shown in the cartoon Fig. 6.2, but rather have sinusoidal density fluctuations along the axis normal to the layers. X-ray diffraction studies[109] show that the density expression as shown is quite accurate, and in fact there are often no higher order sinusoidal terms. Despite this somewhat fluid density change throughout the film, the positional order is strong enough to induce a number of crystalline properties, as will be discussed later.

\subsubsection{Nematic}

The most common mesophase is the nematic phase, whose name originates from the Greek nematikos for "thread-like". This is because the molecules tend to align themselves as though they were a strand of rope. This high degree of orientational ordering but no positional ordering means all molecules are aligned toward the same direction but have random position with respect to each other, as can be seen on the left in Fig. 6.2.

Nematic phases are often used in liquid crystal displays. Their lack of positional order allows them to flow nearly as freely as isotropic liquid while still being easily aligned to external electric or magnetic fields. Their similarities to liquid give them useful properties for fluid dynamics studies, and their characteristics as uniaxial crystals have also been utilized for these and other experimental purposes.[109]

Though widely used in applications where fine electric or magnetic response is desired, 


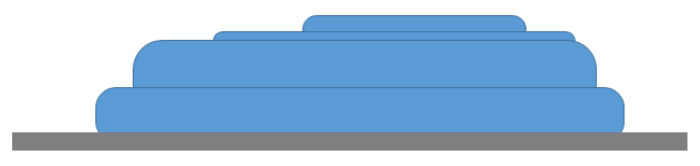

Figure 6.3: Cartoon of a smectic phase liquid crystal droplet placed onto a substrate. Rather than spreading out as a water droplet might, the volume will spread into stacks with points of rapidly varying thickness that resemble solid material cleaves.

nematic liquid crystals have limited utility as film targets. The lack of orientational order makes film formation with an aperture difficult, if possible at all. Even when a film is formed, thickness consistency across the film is much worse than for a film made from the smectic phase, to be discussed in the next section.

\subsubsection{Smectic}

The word smectic comes from the Latin smecticus, which translates to "soap". Indeed dish soap in water is perhaps the most commonly encountered lyotropic (or concentrationbased) liquid crystal, where the long-chain lipid molecules of the detergent can organize into semi-crystalline structures if their concentration is correct.

Smectic molecules tend to arrange themselves into layers with both orientational and positional order, as can be seen in the right of Fig. 6.2. In a perfect smectic phase each layer has the same director orientation. One way to distinguish between types of smectic mesophases is the angle between global director orientation and the layer normal direction. For example, the smectic A phase has the molecule director aligned with the layer normal, while smectic $\mathrm{C}$ has some set angle between these directions.

This positional order has profound effects on the behavior of the liquid crystal. Foremost, the tendency of the molecules to layer themselves provides rigidity to films formed from this phase. If dropped onto a flat surface a smectic liquid crystal will naturally form into shelves of sharply differing heights, as indicated in Fig. 6.3.

Other smectic mesophase types have different patterns of orientational order within one layer. The smectic B phase has in-plane orientation where the molecules arrange themselves into a hexagonal pattern. This so-called bond-orientational order often does not extend farther than a few tens of $n m$, and so is not thought to be useful for liquid crystal films as laser targets. Still other molecules exhibit a long-range three-dimensional order-these are called crystal mesophases, and only exist as phases that are too solid to be used as film targets.[109] 


\section{Orientation of molecules}

The smectic A phase, as well as the nematic phase, are uniaxial crystalline phases. Smectic $\mathrm{C}$, on the other hand, is biaxial. This has important consequence on the use of liquid crystals as polarizing media, which defines their utility in displays. In the smectic $\mathrm{C}$ phase molecules tend to orient their long axis, or $z$ for this discussion, in a preferred angle with respect to the layer normal. The other two molecular axes tend to orient themselves in a particular position as well, although this can have long-range fluctuations.[113]

The consistency of molecular orientation in a smectic phase can be described by another order parameter, $D$ :

$$
D=\left\langle 3 / 2 \sin ^{2} \theta \sin ^{2} \phi-1 / 2\right\rangle-\left\langle 3 / 2 \sin ^{2} \theta \cos ^{2} \phi-1 / 2\right\rangle
$$

where $\theta$ and $\phi$ are the axial and azimuthal angles in spherical coordinates. The first term of $D$ is the angle between the molecular $y$-axis and the director, and the second term is for the $x$-axis. This quantity describes the difference in the average preferred molecular $x$ and $y$ directions, and is typically less than 0.1 for a smectic phase liquid crystal. This means molecular orientation deviation is uncommon throughout the liquid crystal volume.

Much research involves the behavior of smectic liquid crystals around locations where the director varies locally and thus is undefined.[109, 113, 114] Regions of undefined director within a liquid crystal volume manifest as points, lines, or sheets-the most typical type of defects are the line variety, which are called disclinations. Disinclinations can form around defects in the film substrate, or dust particles if no substrate is present. These can also be induced artificially via thermal gradients, or from gradients in an applied electric or magnetic field. Sheet defects tend to spread out and be continuous over a slab containing the sheet, rather than being discontinuous as a set of points.

These defects in director orientation are important for a liquid crystal volume or film because they influence the optical qualities of the system. For example, the uniformity of the director across the liquid crystal can be thought of as a free-energy state, similar to a harmonic oscillator.[109] Defects in the director manifest as splays (an angular spreading of directors), twists (director rotation within a smectic layer), and bends (director rotation between smectic layers). These higher energy states tend to relax over time, although importantly perfect orientation is rarely the case without surface treatment for the confining boundaries of the liquid crystal, or the application of an external field. Advantageously, the structure of the smectic phase tends to repel bends and twists.[113]

\section{Structural effects}

The details of liquid crystal phase transitions depend critically on steric effects and the van

der Waals interactions that vary depending on the considered molecular axis. Crystalline 
phases have the most order, directly resembling conventional crystals but with a few extra rotational degrees of freedom, for example. Increasing temperature slightly can allow for bonds to be broken in one direction while they are maintained in others, since the bond strength differs due to the atomic constituents and their extent in the various molecular directions. For example, liquid crystals that exhibit the smectic phase typically have long lateral substituents which can intertwine to aid in the layering inherent to the smectic phase. Meanwhile, it is the lateral attraction, typically from dipole-dipole interactions, between the rigid, central molecular structures that aid in molecular packing, stabilizing the smectic phase.[109]

Smectic A is the most common smectic phase because it is the most disordered, with smectic $\mathrm{C}$ being the most common among the tilted director phases for the same reason. An illustrative example can be taken from the phases exhibited by the various cyano-biphenyl liquid crystals, to which $8 \mathrm{CB}$ belongs. Table 6.1 shows the phases exhibited by the different molecules and their transition temperatures.[109] C refers to the solid crystalline phase and I is the isotropic liquid phase, with $\mathrm{S}_{A}$ and $\mathrm{N}$ the smectic $\mathrm{A}$ and nematic phases, respectively. Each of these liquid crystal molecules shares the same biphenyl core structure with a cyano terminal group, and then has a varying length alkyl tail for its other end. For a given molecule each column represents the transition temperature from that phase to the next highest (less ordered) phase exhibited. As this tail length increases the temperature of the crystal-isotropic phase transition decreases, until 4-cyano-4'-pentylbiphenyl (5CB) when a nematic phase can be observed. This is because a sufficiently long alkyl tail will intertwine with those from neighboring molecules and aid in the formation of a thread-like nematic structure. Further increasing the tail length also strengthens the tendency for the core structure to pack together laterally, until eventually the more ordered smectic A phase is observed for $8 \mathrm{CB}$. Still further lengthening of the alkyl chain eventually prevents the formation of a nematic phase, although at the cost of increasing the temperature necessary to unpack these long chains from their crystalline phase. The tradeoff between these stabilizing and transition temperature increasing effects is an optimum smectic phase range for $8 \mathrm{CB}$, which conveniently encompasses room temperature.

The smectic layer size is determined by the length of the rigid part of the molecule, which always contains the central core and sometimes the lateral structures. For $8 \mathrm{CB}$ the layer thickness is $2.7 \mathrm{~nm}$. This layer spacing typically does not change with temperature within one smectic phase. Critically, any birefringence exhibited by a liquid crystal comes entirely from the core molecules, and will be diluted when long end chains exist to tangle up and reduce the consistency of the structure. For example, biphenyl cores like those in 8CB typically result in optical anisotropies around 0.2.[109]

Another important physical feature resulting from the molecular structure is the extremely low vapor pressure possible with certain types of central structure. For example, 
Table 6.1: Phase transitions of cyanobiphenyl liquid crystals. The listed value is the temperature at which transition from the phase in that column occurs. $\mathrm{C}$ is crystalline, $\mathrm{S}_{A}$ is smectic A, N is nematic, and I is isotropic (liquid) phase. Modified from [109]

\begin{tabular}{llcccc}
\hline \multicolumn{5}{c}{ Compound } & \multicolumn{5}{c}{ Transition Temperatures $\left({ }^{\circ} \mathrm{C}\right)$} \\
\hline Name & Formula & $\mathrm{C}$ & $\mathrm{S}_{A}$ & $\mathrm{~N}$ & $\mathrm{I}$ \\
$1 \mathrm{CB}$ & $\mathrm{CH}_{3}$ & 109.0 & - & - & $*$ \\
$2 \mathrm{CB}$ & $\mathrm{C}_{2} \mathrm{H}_{5}$ & 75.0 & - & - & $*$ \\
$3 \mathrm{CB}$ & $\mathrm{C}_{3} \mathrm{H}_{7}$ & 66.0 & - & - & $*$ \\
$4 \mathrm{CB}$ & $\mathrm{C}_{4} \mathrm{H}_{9}$ & 48.0 & - & - & $*$ \\
$5 \mathrm{CB}$ & $\mathrm{C}_{5} \mathrm{H}_{11}$ & 24.0 & - & 35.0 & $*$ \\
$6 \mathrm{CB}$ & $\mathrm{C}_{6} \mathrm{H}_{13}$ & 14.5 & - & 29.0 & $*$ \\
$7 \mathrm{CB}$ & $\mathrm{C}_{7} \mathrm{H}_{15}$ & 30.0 & - & 43.0 & $*$ \\
$8 \mathrm{CB}$ & $\mathrm{C}_{8} \mathrm{H}_{17}$ & 21.5 & 33.5 & 40.5 & $*$ \\
$9 \mathrm{CB}$ & $\mathrm{C}_{9} \mathrm{H}_{19}$ & 42.5 & 48.0 & 49.5 & $*$ \\
$10 \mathrm{CB}$ & $\mathrm{C}_{10} \mathrm{H}_{21}$ & 44.0 & 50.5 & - & $*$ \\
\hline
\end{tabular}

$8 \mathrm{CB}$ has two linked phenyl groups as its core, which will interact strongly with the phenyl groups of an adjacent molecule. This strong bonding leads to vapor pressures as low as $10^{-9}$ for $8 \mathrm{CB}$, allowing its use in the high vacuum target chambers necessary for laser-plasma experiments.

Significant chemical and physical study of the steric properties of liquid crystals has been performed, but is only cursorily provided here as it applies to the use of liquid crystals in making smectic phase films. The text by Collings and Hird[109] provides a thorough introductory discussion on the nature of various molecular constituents and their effects on liquid crystal phases and their transition temperatures.

\subsubsection{Chiral}

Both the smectic and nematic phases discussed so far have consistent director orientation, except at localized defects. There are other phases that exhibit long range patterns in the director pointing: in these phases the pattern is often a rotation of the director orientation as a function of depth, as shown in Fig. 6.4, tracing out a helical pattern. These phases are known as cholesteric because they were originally observed in derivatives of cholesterol molecules, but are also called chiral phases because of the handedness of the director rotation. The pitch distance $p$ is the depth required for the director angle to undergo a full rotation. However, since antiparallel directors have equivalent optical effects the half pitch distance $p / 2$ is most relevant. This pitch can vary as a function of temperature in thermotropic liquid crystals or with concentration of a chiral element in an otherwise non-chiral liquid crystal mixture.

Chiral phases arise primarily due to steric effects in the molecules: a polarizing terminal 


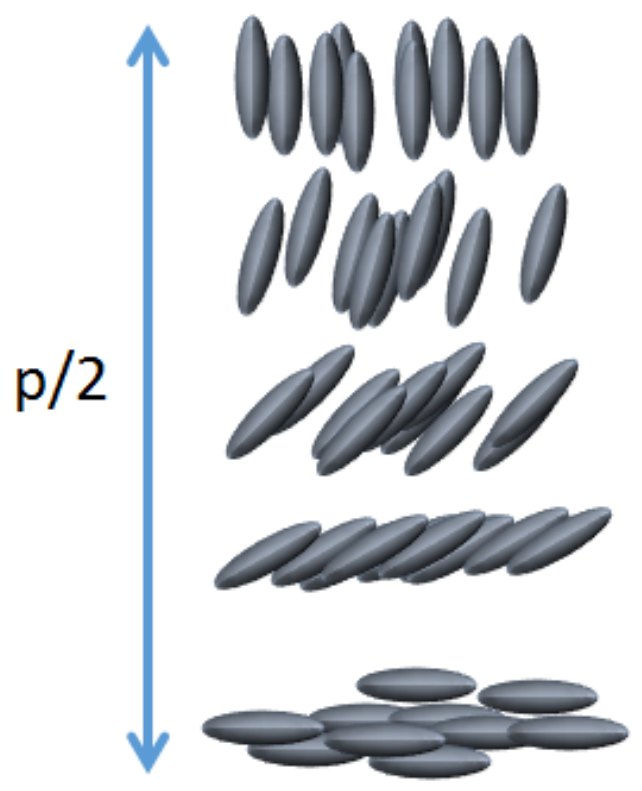

Figure 6.4: Cholesteric or chiral liquid crystal phase. Here each smectic layer has a different director orientation, and the pattern repeats itself after the pitch distance $p$.

chain or lateral substituent will tilt away from its neighbor, causing a regular molecule rotation throughout the liquid crystal volume. These chiral rotations can occur as a bulk effect in a nematic phase, or layer-by-layer in a smectic phase. As such chiral phases are often denoted by listing the appropriate base phase with an asterisk to denote the chirality, as in the smectic $\mathrm{C}^{*}$ phase.

Chiral phases have unique optical properties when the pitch distance is on the order of the wavelength of light used to probe the liquid crystal-namely, matching pitch distance to wavelength results in the Bragg reflection of that light from the liquid crystal film. In this way chiral liquid crystals can be used as a thermometer: certain wavelengths of white light incident onto the film will reflect only at certain temperatures. ${ }^{15}$ These thermochromic liquid crystal devices can have a resolution of $0.1^{\circ} \mathrm{C}$, and are commonly used as inexpensive disposable thermometers when a more precise measurement is not required.

In addition to the rejection or transmission of optical light by its frequency, chiral liquid crystals can also be used to manipulate the polarization of light. For example, a chiral liquid crystal that is initially uniaxial can be shifted into a biaxial organization by the application of an electric field, with the degree of re-organization dependent on the magnitude and direction of that field.[115]

\footnotetext{
${ }^{15}$ In fact this is the method of operation of the "mood ring" toy popularized in the 1970's: the small amount of heat could be applied by touching a glass or plastic covering over the liquid crystal film. This heat was enough to increase the pitch distance of the film, resulting in varying reflected colors as the film was heated and then again as it cooled down and the pitch distance contracted.
} 
This effect and others make chiral liquid crystals the most common candidate for use in displays. Here the liquid crystal layer is placed between glass plates, and this stack is placed between crossed polarizers. The surface of the glass can be treated such that the immediately adjacent liquid crystal molecules will line up in a certain way there so that the beginning of the helical rotation is known. In this way the orientation of the liquid crystal molecules is set by its environment for the case when the voltage is off. In simple displays like those on digital watch faces or calculators, this neutral liquid crystal orientation is often set to shift the polarization of incoming light such that it is allowed through the second polarizer. Providing a bias to the liquid crystal layer will then cause a reorientation of the molecules which then further shift the polarization of incoming light such that it is rejected by the subsequent polarizer.[109] ${ }^{16}$

\subsection{Freely Suspended Films}

Smectic mesophases can be formed into what are known as freely suspended films.[113, 116] Here the surface tension inherent to these phases can cause a thin film to form within a frame, just as a soap film will form within a ring dipped into a soap solution. Critically, these freely suspended films are formed with no substrate the front or back of the film, in contrast to the films used more typically in displays. The freely suspended nature of these films is useful to various fluid dynamics experiments studying the properties of convective flow, often in the presence of electric or magnetic fields.[117-122] It also allows their use in laser experiments where both the incoming and outgoing directions need to be open for laser interaction and the resulting particle acceleration.

The smectic phase is ideal for producing freely suspended films because of its favorable surface tension. This has been measured for a number of liquid crystals.[113] For all types of smectic phase-even those with longer range three-dimensional order-the surface tension has been found to be greater than the elastic modulus of the material, which suggests the rigidity of the freely suspended film. For example, the surface tension of the liquid crystal used for this work, $8 \mathrm{CB}$, is around 60 dynes $/ \mathrm{cm}$ (or, equivalently, $60 \mathrm{mN} / \mathrm{m}$ ), decreasing slightly with temperature and increasing linearly with film thickness.[113]

From previous studies on freely suspended films, a number of basic structural elements can be determined.[113, 116, 123] First, the meniscus region that connects liquid crystal film to its frame serves as a reservoir for molecules during the film formation process, or during the stretching of a film formed in a changeable frame. The transfer of volume from meniscus to film is optimized when the meniscus is as fluid as possible, meaning in the Smectic A or

\footnotetext{
${ }^{16}$ This is why non-backlit liquid crystal displays like those in calculators will have black numbers-here the applied voltage is causing rejection of incoming light so that no light is hitting the reflective rear surface of the display. In lit displays like those on microwaves, for example, the reorientation by voltage allows light to pass through the system, which is why the digits appear lit in this case.
} 
$\mathrm{C}$ state. In general films are metastable with respect to thickness, with thinner regions of a film almost always eventually overtaking the entire aperture. In the absence of impurities, the lifetime of a film is near infinite, at atmospheric pressure or under vacuum. A faster film formation rate increases the likelihood of the nucleation of a thin region of film, which will eventually overtake the entire area. Finally, several domains of different thicknesses can arise within a film, often intersecting at some impurity (e.g. dust) within the liquid crystal volume.

For proper film formation, a number of considerations must be met. First, the liquid crystal must be heated to somewhere within its smectic phase-this done historically by holding the film and frame within a small oven with controllable temperature,[114] but merely heating the frame itself will suffice. The frame is best polished to a mirror finish, free of nicks and scratches in the aperture edge which will result in director alignment defects and weaken the film in general. The thickness produced depends broadly on the wiper rate, amount of liquid crystal volume applied, and the temperature, with thinner films produced for faster wiper speeds, higher temperatures within the smectic range, and smaller volumes. The details of film formation will be presented in Chapter 7 .

In a freely suspended film the air-surface interface has a similar but weaker effect on the surface director orientation as compared to that of a treated glass substrate. This surface-induced orientation has a diminishing effect on deeper layers of the smectic film. The layer depth distance over which this orientation persists is known as the correlation length. Analogously to the skin-depth effect with light penetrating an opaque material, this surface-induced liquid crystal director orientation decreases exponentially with the correlation length as a scale factor.

Though this surface-induced order may not persist initially much further than the correlation length, a process of annealing can over time orient all molecules in the film appropriately. In essence bulk deformations are not energetically favorable within the smectic liquid crystal lattice, and the molecules will reorient to the correct positions eventually. These methods can be enhanced either by careful, clean film formation (which minimizes the initial nonuniformities), or by moving the formed film through a phase transition repeatedly. This must be done with some care since the change in surface tension at a less ordered phase can destroy the film. Alternatively, order nonuniformities will be removed by lattice fluctuations over a sufficient amount of time. Previous efforts describe that the thinnest films (for layer number $N<10$ ) need thirty minutes to anneal properly, and thicker ones can require hours up to more than a day to do so.[114] Films that persist for these lengths of time have increased rigidity and resilience, and will maintain their annealed thickness indefinitely if left relatively undisturbed. An example is shown in Fig. 6.5-this film was formed in a $6 \mathrm{~mm}$ inner diameter washer as part of this work, and once its thickness had settled the film remained in this state at that same thickness a year later. 

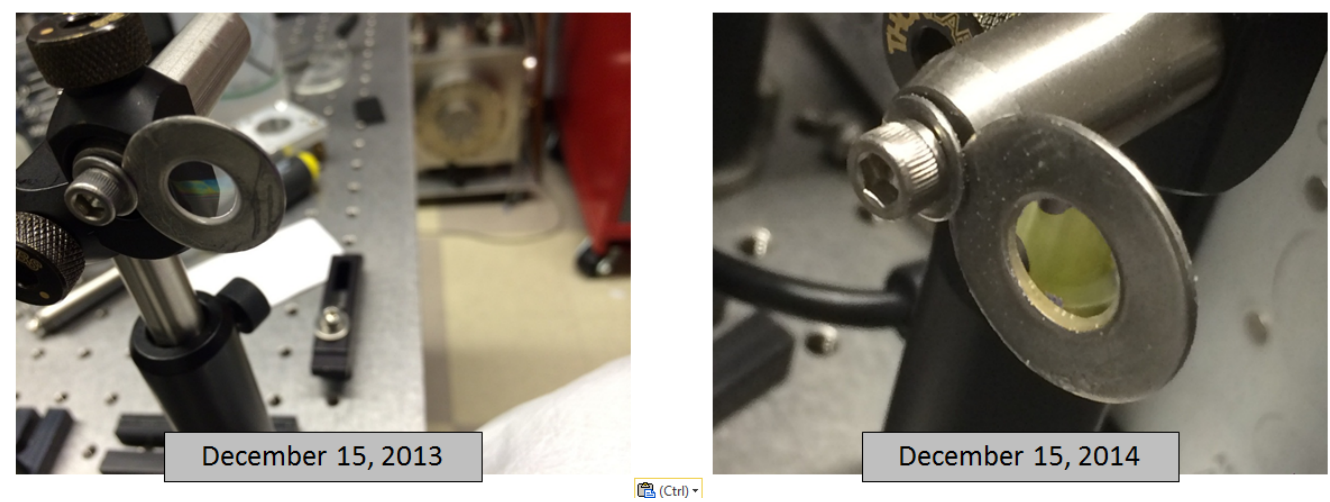

Figure 6.5: A 8CB film formed within a $6 \mathrm{~mm}$ inner diameter washer. Initially there was a vertical thickness gradient from excess volume (left), but once this excess wicked away the annealed film remained that thickness $(300 \mathrm{~nm})$ more than a year later (right).

For each temperature $T$, there is a maximum layer number $N(T)$ below which the film is metastable and above which it thins systematically, beginning typically from an inner layer.[113] This temperature/layer stability also has an effect on the so-called critical radius of a film island or hole, below which this new region will collapse. Far from $N(T)$ this radius is $\sim$ tens of $\mu m$, in which case spontaneous nucleation is highly improbable. Even though film thinning is energetically favorable in this case, it is impossible to actually occur. For $N<10, r_{c}<1 \mu m$, meaning these films are much less stable.

The details of smectic freely suspended liquid crystal films presented here are oriented on the generation of relatively thin films, often $<100$ layers thick. Counterintuitively, it is much easier to form a minutely thin liquid crystal film than one on the few- $\mu m$ scale-this is opposite to the behavior of soap films, for example, where very thin films are difficult to produce and have short lifetimes. It is important to note that this extreme thinness is desired for the use of liquid crystals in a fluid dynamics capacity,[123] and as such significant effort into generating $\mu m$-scale freely suspended films had not previously been warranted. However, the optimization of laser-target interactions, specifically those involved with high intensity laser ion acceleration, necessitates thicknesses on the order of $1 \mu \mathrm{m}$. Increasing the thickness of a liquid crystal film by adding smectic layers is in fact a difficult process involving refinements on the methods detailed here and the inclusion of some new techniques. The details of forming such films will be discussed in the next section. 


\section{Chapter 7}

\section{Freely Suspended Liquid Crystals Film Formation Techniques}

\subsection{Overview}

This section discusses the formation of liquid crystal films within rigid frames with no substrate, or freely suspended films. While liquid crystals in their smectic phase can be formed into such films with minimal effort, manipulating formation to include thickness control is not straightforward. However, correct formation technique allows film thickness variations between $10 \mathrm{~nm}$ and more than $10 \mu \mathrm{m}$. Here these formation techniques will be presented, beginning with the simplest to form a film quickly and culminating in the processes necessary to achieve fine thickness control. A discussion of the measurement of these films is also included.

\subsection{Previous methods of film formation}

Freely suspended liquid crystal films have traditionally been used as two dimensional devices for fluid dynamics experiments. The nature of smectic layer formation combined with the surface tension of some liquid crystals can routinely result in films $\mathrm{cm}$ in both transverse directions but only tens of $\mathrm{nm}$ thick. This environment is ideal for studying two dimensional convective flows, for example, as well as the nature of thin film liquid crystal layer response to applied voltages or fields.

These films are typically formed merely by placing some small volume of smectic liquid crystal next to an aperture in a rigid frame and then drawing this volume across the aperture with a non-absorbing wiper like a razor blade, as depicted in Fig. 7.1. Volumes necessary for this film formation are quite small: the volume of a $500 \mathrm{~nm}$ thick film formed within a $5 \mathrm{~mm}$ diameter circular frame is about $10 \mathrm{~nL}$. Since all applied liquid crystal may not reach the aperture depending on the nature of the wiping, volumes of $100 \mathrm{~nL}$ to $1 \mu \mathrm{L}$ are best when attempting to make a film by hand. For instance, Fig. 7.2 depicts various 


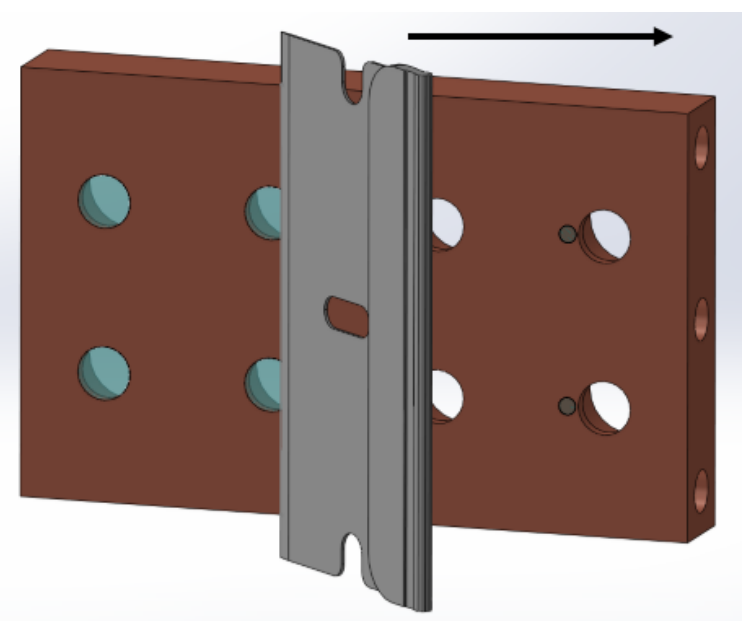

Figure 7.1: Due to the beneficial surface tension of the smectic phase, liquid crystals can be formed simply by wiping a small volume across an aperture in a rigid frame, as depicted here. Volumes as low as $100 \mathrm{~nL}$ are sufficient to make films in apertures of diameter between a few $\mathrm{mm}$ several $\mathrm{cm}$. The wiper can be any non-absorbing material with a flat edge to ensure uniform contact with the frame.

volumes of the liquid crystal 4-cyano-4'-octylbiphenyl (8CB) placed next to $4 \mathrm{~mm}$ diameter apertures machined into $6 \mathrm{~mm}$ thick copper. Lightly dragging any of these volumes across their adjacent aperture with a standard razor blade held in hand will nearly always produce a thin film. The range of possible thicknesses of each film depends on the volume used, but it is not necessarily the case that a greater volume would result in a thicker film due to the nuances of film formation to be described below.

Experimenters wishing to have more controlled film formation techniques often construct an apparatus which will drag the razor wiper across an aperture evenly with a variable speed stepper motor.[123] Such a device removes much of the inconsistencies inherent to hand drawing of films and at the same time adds wiper speed as a tunable film formation parameter. In general thinner films result from slower speeds on such devices, but this is ultimately apparatus-dependent. Additionally, reverse wiper motion mid-draw has been shown to change the smectic film thickness locally if an undesired thickness appears to be forming.[123]

Though this provides some thickness control, trial and error is typically the only way to finally arrive at a desired thickness, especially if accuracy better than $100 \mathrm{~nm}$ is desired (which is most certainly the case for the thinnest films). Additionally, wiper speed and redrawing as described above will not on its own allow the formation of films above a few hundred $\mathrm{nm}$ thickness. This is no problem to the majority of fluid dynamics experiments since a $\mathrm{nm}$ thick film provides an optimum two-dimensional environment, but is certainly an issue when these liquid crystal films are to be used as laser targets. 

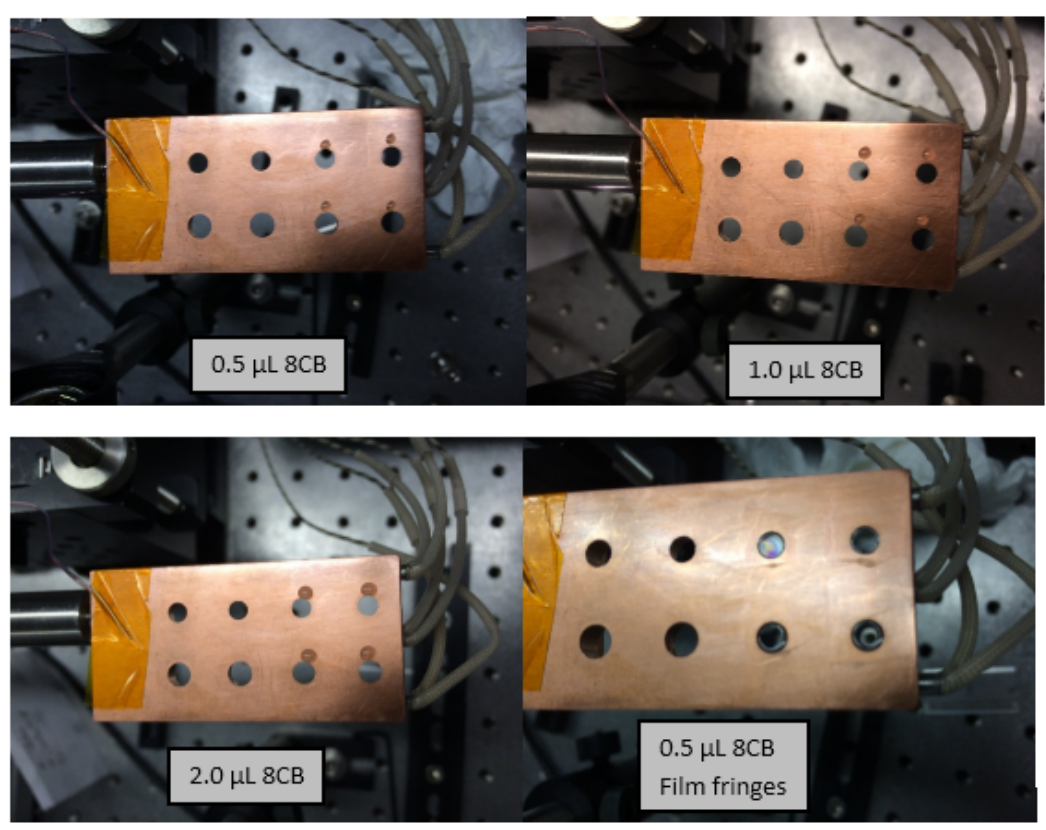

Figure 7.2: Four different volumes applied next to $4 \mathrm{~mm}$ apertures in a copper frame. These volumes were metered out by a precision syringe pump with a $25 \mu L$ syringe. In general more volume results in a thicker film, with $0.1 \mu L$ or less being sufficient for film formation.

\subsection{Thickness measurement of liquid crystal films}

When making freely suspended films to be used as targets or for other applications where a certain film thickness is required, the measurement of that thickness can be achieved via a number of methods. Typical suggestions are to use some form of ellipsometry for films above 100 layers thick, a reflected color measurement for films between 20 and 100 layers, and a reflected intensity measurement for those films under 20 layers.[113, 114].

With possible thicknesses from $\mathrm{nm}$ to $\mu \mathrm{m}$, freely suspended liquid crystal films are perhaps best measured with optical interference techniques. A simple version of this is simply to view the color of white light reflected from a film. Interference between light reflected from the front and rear film surface will produce colors directly dependent on the film thickness-this is the same effect that gives oil slicks and soap bubbles their color. Charts of color as a function of thickness can be produced if the index of refraction, viewing angle, and light source spectrum are known, as shown in Fig. 7.3.

This interference method loses some utility for films thinner than $100 \mathrm{~nm}$. At this thickness and below the wavelength of light that will constructively interfere drops out of the visible range and into the ultraviolet. Since there is a $\pi$ phase shift upon reflection from the front film surface (lower to higher index transition), visible light reflected from this 


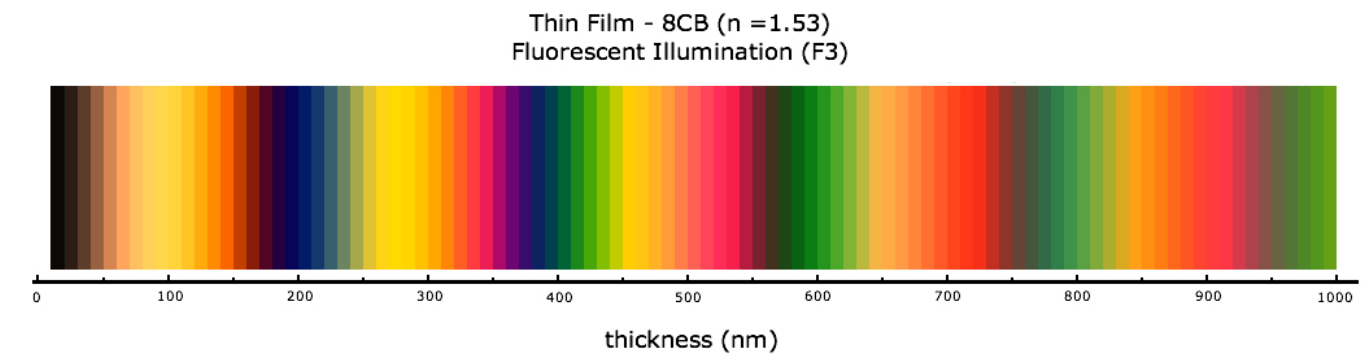

Figure 7.3: Chart showing the reflected color from a thin $8 \mathrm{CB}$ film with respect to thickness. Sub-50 $\mathrm{nm}$ films are black films, where all visible wavelengths destructively interfere upon reflection. Increasing thickness from these dark films leads to a series of color bands, eventually resulting in primarily pink and green bands due to the combination of colors interfering constructively at those large thicknesses. Image credit RJ Hanna.

position will be progressively closer to exactly out of phase with visible light reflected from the rear surface as the film thickness decreases from around $100 \mathrm{~nm}$. Around $50 \mathrm{~nm}$, all visible wavelengths will destructively interfere, which is why films in this thickness regime are classified as black films. Note that only reflected light destructively interferes, so the films will only appear black if there is no surface close behind the film from which light transmitted through the film will reflect.

As seen in Fig. 7.3, the color around $100 \mathrm{~nm}$ is fading from yellow to light gray, and then getting progressively darker until it is black for the thinnest films. The reflectivity from the film changes constantly throughout this thickness region, however, and so can be used to determine thickness despite the lack of color. Films below $10 \mathrm{~nm}$ are classified as Newton Black Films, and exhibit some different properties because they are chiefly comprised of two surface layers with little or no bulk material in between.[116]

As the film thickness increases above $100 \mathrm{~nm}$, bands of somewhat repeating colors are observed, since optimum constructive interference of one color film can occur for thicknesses $d$ that satisfy

$$
2 n_{f i l m} d \cos \beta=(m-1 / 2) \lambda .
$$

This is due to the $\pi$ phase shift upon reflection from the front film surface, since $n_{\text {air }}<$ $n_{\text {film }}$. At thicknesses above $0.5 \mu \mathrm{m}$ there are multiple wavelengths that constructively interfere, such that the combination of these colors tends to produce films that look green or pink.[124]

This sort of color measurement can pose some difficulties. For one, the color observed is dependent on the angle of incident light, meaning that a consistent position must be used for an eye or camera that is judging film thickness based on the color. The repetition of 
color bands with increasing thickness presents another problem: a color chart like Fig. 7.3 is useful for films in the sub-100 $\mathrm{nm}$ regime or if a good estimate of the film thickness is known prior to measurement, but otherwise it is not possible to determine the precise thickness of say a blue film between the possibilities of $200 \mathrm{~nm}$ and $375 \mathrm{~nm}$. This problem is exacerbated for pink or green films, which have many possible thicknesses from $500 \mathrm{~nm}$ up through a few $\mu m$. In essence, the order of the interference is not known here, and must be determined.

This issue can be solved by one of a few methods: often thin films have varying thickness across their substrate, or can be made to have temporarily varying thickness, e.g. by tilting a horizontal soap film such that gravity pulls the film to be thicker at the lower end. The result of such manipulation is to provide a reference point by which another thickness can be judged via a counting of the bands across this continuously varying region. Unfortunately, if uniform thickness is desired-as it is in the use of liquid crystals as ultra-intense laser targets, where as discussed the thickness critically determines the physics of ion acceleration-such a reference point is antithetical to the desired application of the film.

Another possible solution is to use one wavelength of light in the form of a laser, observing the interference fringes of this light that are visible in reflection. In this case the angle of the incident light can be varied within one beam by using a short $F / \#$ focusing setup. For a $F / 1$ optic focusing light at $45^{\circ}$ the incidence angles range from $18.4^{\circ}$ to $75.6^{\circ}$. A number of constructive and destructive interference fringes will be visible in reflection from a thin film based on its thickness, as seen in Fig. 7.4. While this method has the advantage of operating in a reflection mode, the fast focusing required limits the minimum discernable distance to a $\mu m$ or so, and the close lens-target distance necessary makes this a non-optimal technique even for thick film measurement.

An alternative solution, then, is to reflect a broad spectrum of light from the thin film and measure the resulting constructive and destructive interference. This spectral interference technique will work on a single-thickness film and can be quite accurate if the index of refraction of the film material is already known. This is the method used by the Filmetrics spectral reflectance device used in experiments as part of this work. Here a known white light spectrum from a halogen bulb $(350 \mathrm{~nm}-1050 \mathrm{~nm})$ is measured after reflection from a known reference material (e.g. BK7 glass) followed by reflectance measurement from the unknown thickness film. This reflection setup and a sample measurement spectrum are shown in Fig. 7.5. Here the blue curve on the right is the measured modulation in the input white light spectrum, while the red curve is the result of an iterative algorithm to match this measurement curve from a rough thickness guess.

This measurement requires some knowledge of the index of refraction of the material being measured. Unfortunately the index of refraction of many liquid crystals can change both between mesophases and as a function of temperature within one phase. For example, 


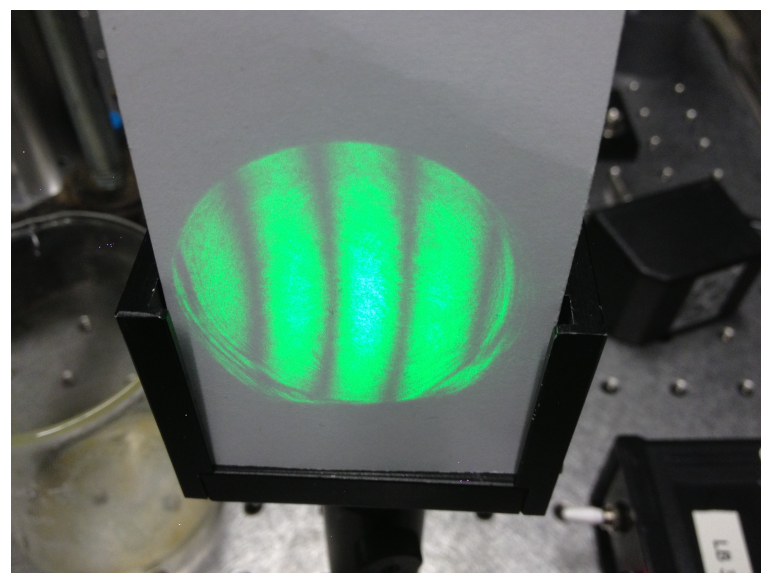

Figure 7.4: Fringes visible in the reflection of a $532 \mathrm{~nm}$ laser with $F / 1$ focusing on an 11 $\mu m$ shard of transparent mica. The fringe number can be correlated to a thickness value once the spread of incident light angles has been determined from the focusing parameters.

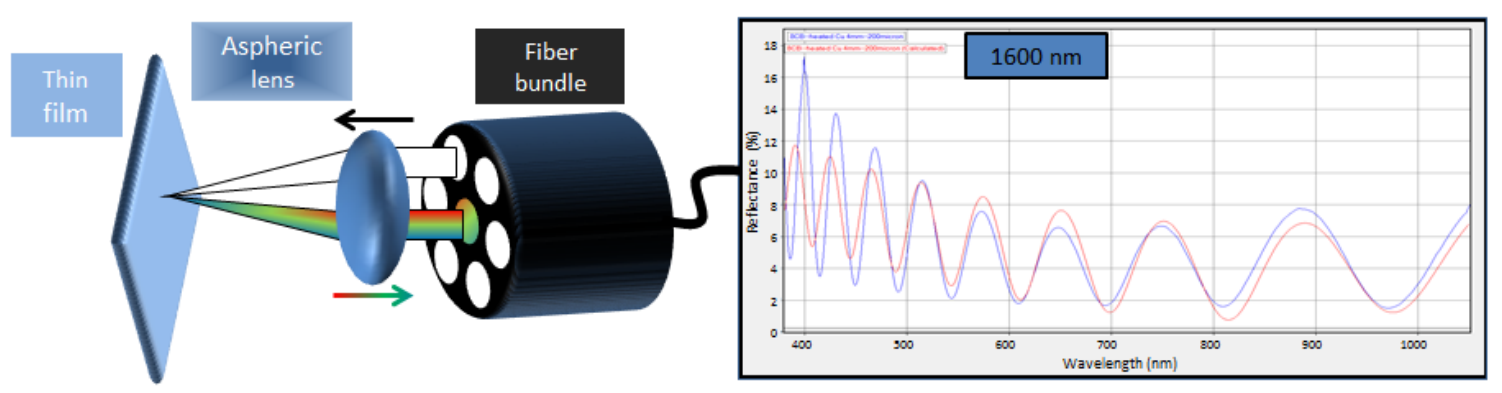

Figure 7.5: Spectral reflectance technique utilized by the Filmetrics thickness measurement device. The graph at the right shows the modulations in the reflected white light (blue) and the results of an iterative algorithm to match this curve based on an initial thickness guess (red). 
the ordinary index of $8 \mathrm{CB}$ will change from 1.68 to 1.67 across its smectic phase, and from 1.67 to 1.63 across the nematic phase.[125] Additionally, the index of refraction can change as more contaminants are introduced into the liquid crystal, and in general is best re-measured on a new sample of liquid crystal and periodically during its use.

Conveniently, this spectral reflectance measurement technique can be done with an integration time of $50 \mathrm{~ms}$, enabling high repetition rate film thickness interrogation. Additionally, the fiber light source can be imaged with a relay telescope over a long distance, allowing this device to measure films at the center of a target chamber while the device is aimed from outside the chamber through an optical port. The details of Filmetrics setup on a chamber will be revisited in subsequent Chapters.

\subsection{Thickness control of freely suspended films}

\subsubsection{Obstacles to uniform film thickness}

Film formation by hand or with a motorized wiper at room temperature $8 \mathrm{CB}$ (which will be in the smectic phase) will typically form a film below $100 \mathrm{~nm}$ thickness. Somewhat counterintuitively, increasing the volume before drawing does not always result in a thicker film. Rather, the additional volume typically distributes within a thick meniscus region where the film connects to the aperture frame. This meniscus is typically opaque at room temperatures because there is no global orientation of the smectic layers within it. In contrast, the central region of the film stretches as it is drawn, with surface tension within the film playing a greater role far away from the frame edge. This often results in a film only several layers thick in the central area of the aperture, surrounded on all sides by a thick, opaque meniscus containing the bulk of the initial volume. A microscope image of a freely suspended 8CB film is shown in Fig. 7.6.

Controlling thickness within this central region relies on temperature modification. Since the surface tension of liquid crystal changes with temperature, induced thermal gradients will cause the flow of some layers over others. For example, if temperature is applied to a film with a meniscus, this region will slowly encroach upon the central thin area as it grows in temperature. The result is often an "amphitheater" film, with concentric rings of different colors (indicating different thicknesses), as seen in Fig. 7.7. In these films the very central region is often still sub-100 $\mathrm{nm}$ as it began, but its area has shrunk significantly. At higher temperatures the remaining meniscus region will become clear-this is the same as the clearing temperature effect first observed by Reinitzer to distinguish liquid crystals from conventional substances.

Thickness nonuniformities can also be generated by excess volume in a vertically held film, as shown in Fig. 7.8. Here liquid crystal in a $4 \mathrm{~mm}$ aperture was formed with enough volume that gravity has pulled some down to the lower region of the film. The result is a 


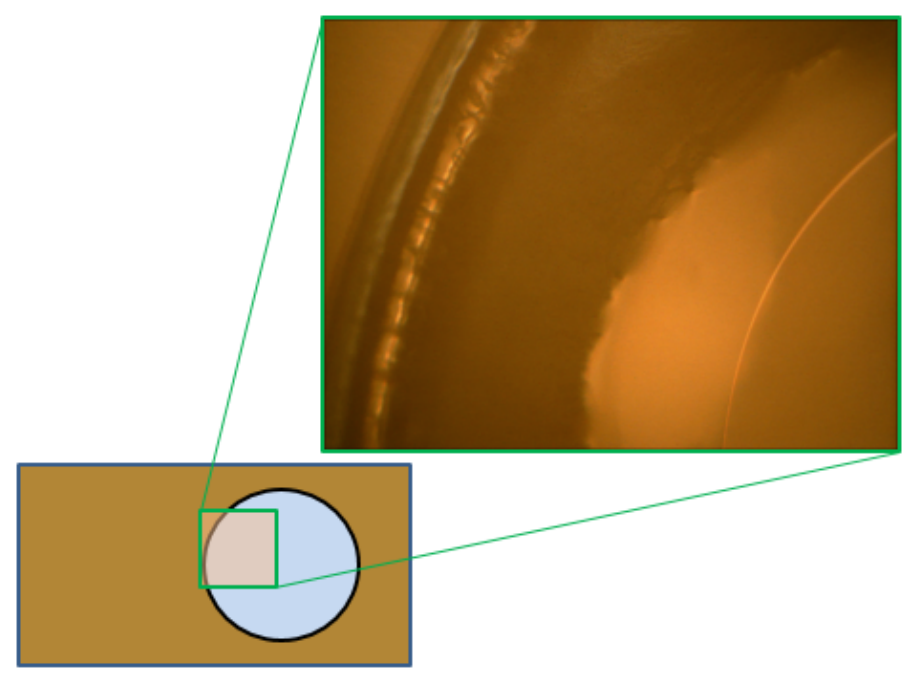

Figure 7.6: $4 \times$ magnification image of one section of a $4 \mathrm{~mm} 8 \mathrm{CB}$ film. Visible is a thin film region in the lower right, a ring of thicker film, and then the opaque meniscus region. Each of these regions is comprised solely of the liquid crystal $8 \mathrm{CB}$, and their varying appearance comes from the changes in thickness and molecular orientation.

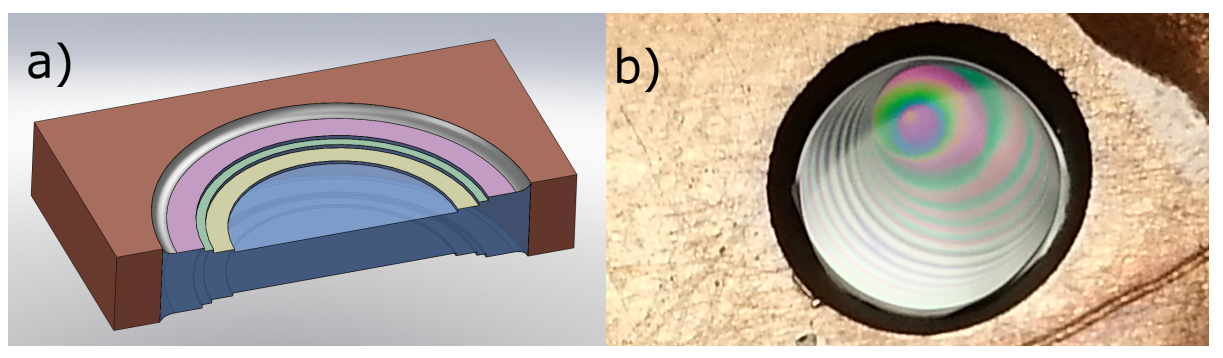

Figure 7.7: Here a so-called "amphitheater film" is shown, where the thickness decreases in radial steps from the outer to inner ring. Depicted are both a) a cartoon of this phenomenon and b) an actual amphitheater film within a $4 \mathrm{~mm}$ aperture. These rings are different in thickness by many layers, giving rise to the distinct bands observed here. It is possible for the thickness range of such a film to span from a few $\mu \mathrm{m}$ at the outer ring to a sub-50 $\mathrm{nm}$ film in the center. 


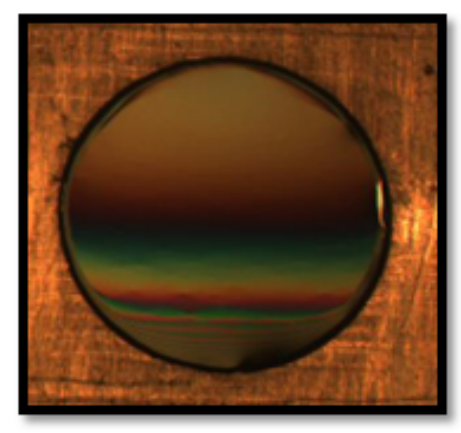

Figure 7.8: Film formed within a frame held vertically. The excess volume of liquid crystal is dragged down by gravity such that the thickness of the film increases lower down within the aperture, as can be seen by the bands of color variation.

vertical thickness gradient visible as horizontal bands of color. Such films are often a few $\mu \mathrm{m}$ thick at the lower region and sub-100 $\mathrm{nm}$ at the top, changing non-continuously with height in the film. This film morphology is in principle usable as a laser target because the color bands can be much larger than the laser spot size, but then only if the thickness gradient is reproducible (or at least controllable) and if it is stable over time. Even if these constraints can be maintained, forming a uniform thickness film is certainly preferable.

Another relevant thickness variation that often occurs as films are drawn is the phenomenon of islands. These are regions of thicker films that float on the substrate of the thin film of liquid crystal stretched between the meniscus ring, as seen in Fig.7.9. Islands can be up to millimeters across and are often several to several tens of layers thick. They can detach from or coalesce into the meniscus region if it is present on a film, but can also be generated during initial film formation. Additionally they are mobile, responding to gravity in the case of a non-horizontal film or to any convective flows set up by air currents or by electric fields within the liquid crystal. Islands will typically move around until they encounter and are absorbed by the meniscus, although for large islands this has a chance to break the film. Though their formation and manipulation constitutes an active area of study for fluid dynamics research,[126] they are undesirable for using liquid crystals as laser targets because of the thickness inconsistency they introduce.

\subsubsection{Achieving uniform film thickness}

To prevent the formation of large meniscus regions and islands, careful control of both the temperature and volume of applied liquid crystal is necessary. The use of a copper frame for superior heat conduction and a precision syringe pump for volume application solves these problems. Temperature control near the smectic/nematic phase transition of $30{ }^{\circ} \mathrm{C}$ is necessary to form a film above the typical room-temperature few-hundred $\mathrm{nm}$ meniscus 


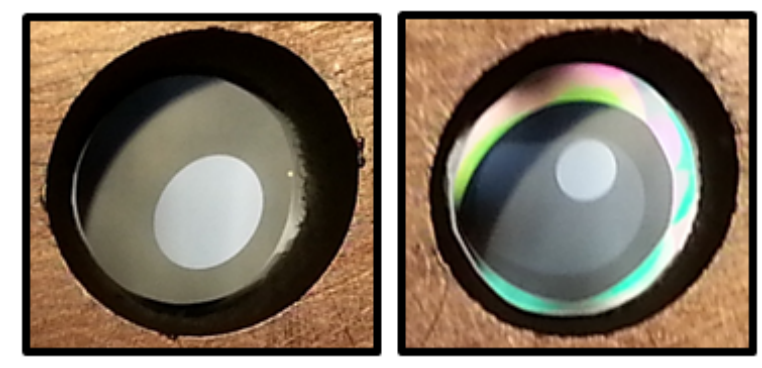

Figure 7.9: A region of thicker liquid crystal floating upon the thin substrate, known as an island, can be seen here. Islands are typically a few $\mathrm{mm}$ across and move around on the overall film surface due to air currents or gravity. The right hand image actually has two island regions forming a tiered thickness area in the center of the film, visible as strong and faint white reflection regions.

regime. Additionally, volumes around and below $1 \mu \mathrm{L}$ will make films as thick as $10 \mu \mathrm{m}$ without producing a large meniscus region, as the long as the appropriate temperature regime is maintained as well.

To achieve appropriate temperature control, a Proportional Integral Derivative (PID) controller was employed on the experiments in this work. Such a device monitors the input from a voltage device, in this case a type $\mathrm{K}$ thermocouple (for its $<0.1^{\circ} \mathrm{C}$ accuracy), and will either toggle a relay switch or provide a $0-10 \mathrm{~V}$ DC current as an output. This can be used to provide a set voltage to resistive cartridge heaters as used here, or to provide variable voltage to a power unit that could run a Peltier cooling block, for example. Here the relay output is used with three $25 \mathrm{~W}$ maximum power, $3 \mathrm{~mm}$ diameter resistive heaters, as seen in Fig. 7.10. These resistive heaters are set to lower than maximum power output by implementing a variable resistance tuner set to $25 \mathrm{~V}$ but that can be changed if additional heating is required. The input temperature and output resistive heating essentially constitute a closed system that the PID controller can manipulate-once an initial calibration test is run, the PID controller can adjust the timing and duration of relay switching such that a desired set temperature can be maintained on the target block within $0.1^{\circ} \mathrm{C}$.

For these experimental setups, precise volume control was implemented via a Harvard Apparatus PHD infuse/withdraw syringe pump (PHD Ultra 2200) equipped with a variety of syringes including $25 \mu \mathrm{L}$ and $1 \mathrm{~mL}$ (Hamilton). Merely $10 \mathrm{~nL}$ of $8 \mathrm{CB}$ is required to form a film, however $100 n L$ is a more typical lower bound given the difficult in drawing the entirety of so small an applied volume directly to the film formation region.

The formation of very thick films, several $\mu m$ and above, requires a large volume of liquid crystal compared to the standard amount, as well as significant heating. The procedure involves placing several $\mu L$ of liquid crystal volume next to an aperture heated to a few ${ }^{\circ} \mathrm{C}$ below the smectic-nematic phase transition. Drawing a film under these conditions will 


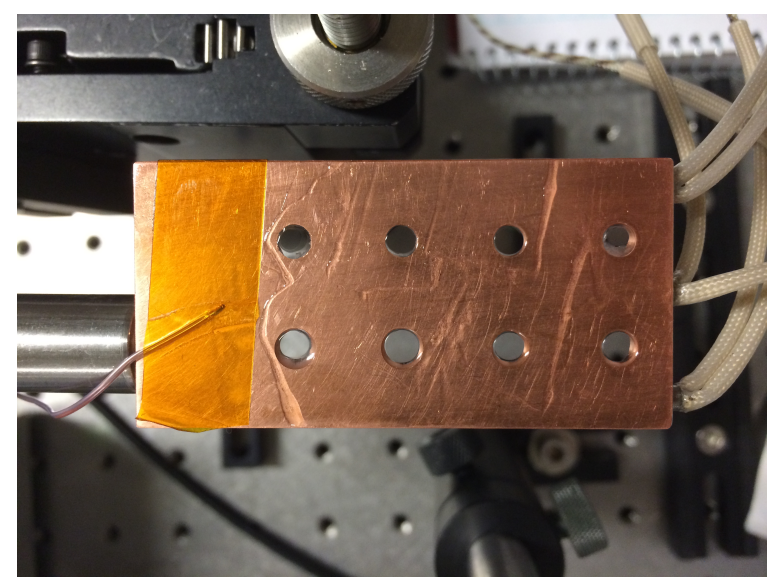

Figure 7.10: Temperature control is required for precise thickness manipulation. This copper frame is $6 \mathrm{~mm}$ thick, allowing inserting of three $3 \mathrm{~mm}$ resistive cartridge heaters to be inserted into the bulk metal, visible at the bottom of the image. A thermocouple can be seen at the top of the frame for temperature monitoring and for control through a PID controller.

result in a large, opaque meniscus layer connecting an inner thin film to the frame edge. Heating this film to just above the smectic-nematic phase transition will cause the meniscus region to first encroach upon the central film area, and then become clear. Once such a film is allowed to cool it will be several to several tens of $\mu m$ in its center. Alternatively, a similar amount of volume placed on the Linear Slide Target Inserter (LSTI) device to be discussed in Chapter 10 can also produce such films.

\subsection{Film frame design}

Multiple frame designs were considered before an optimum one was chosen. For example, Fig. 7.11 shows a number of other materials used as frames for film formation as well as the schematic for the apertures in these frames. Among these copper proved to be superior due to its high thermal conductivity, although films greater than $500 \mathrm{~nm}$ can be made in any of these frames with sufficient application of heat. The thermal conductivity of pure copper is $\sim 400 \mathrm{~W} /(\mathrm{Km})$, which is twice that of aluminum and eight times that of steel.

A further consideration for the frame lies in the nature of the aperture. A series of tests were performed on aperture diameter, thickness, and edge quality. The diameter only has the effect of changing how much perimeter is available for meniscus formation at a given volume of liquid crystal, and as expected changes the range of thicknesses possible with a given volume. Apertures of $4 \mathrm{~mm}$ diameter were found to be the most convenient for formation, although they are not the only film size that works: films have been made in frames as small as a few tens of $\mu m$ (in the form of a copper wire mesh) up to more than 

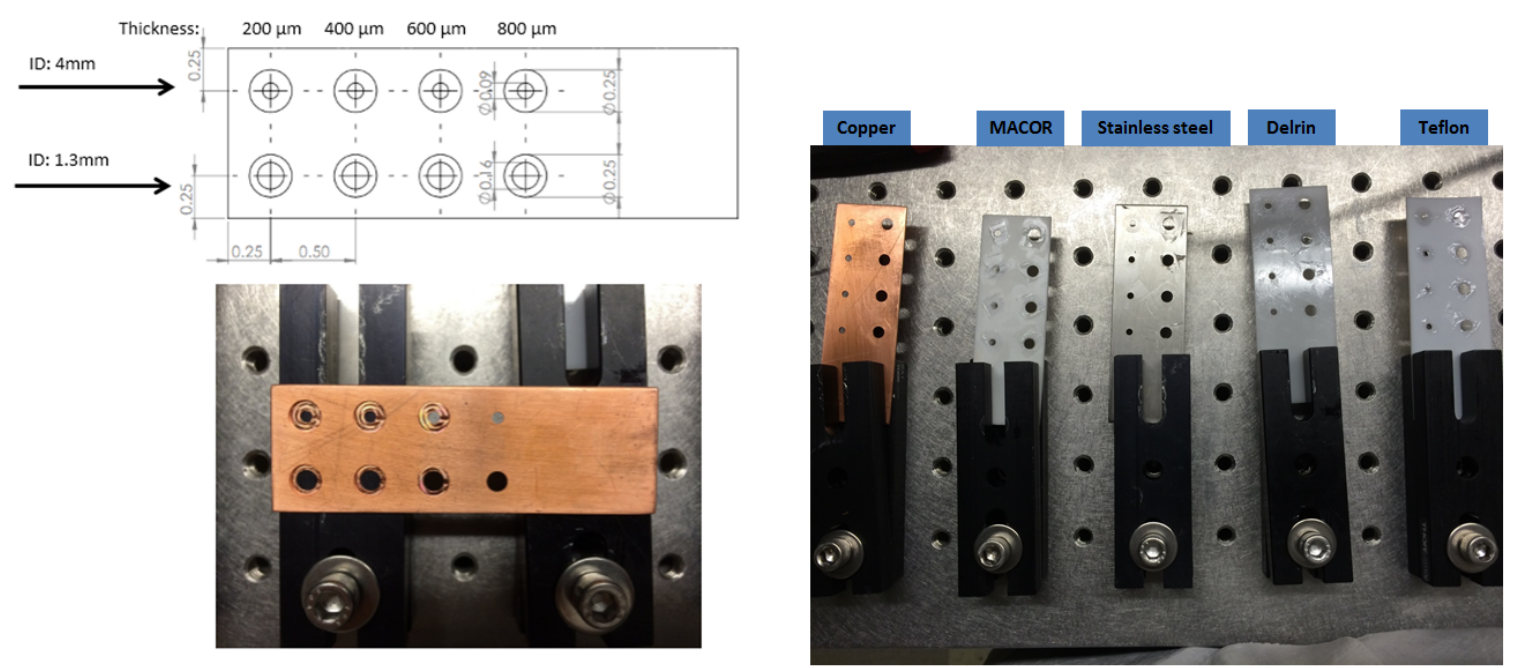

Figure 7.11: Frames for film formation were made with various aperture thicknesses as well as a number of diameters, two of which are shown on this frame.

several $\mathrm{cm}$ across.

Frame thickness was not found to have a large effect on the thickness of film formed. Thicknesses from $200 \mathrm{~nm}$ up to $1 \mathrm{~mm}$ were tested, all with inconsistent film thicknesses as applied volume was varied on each aperture. With no temperature control, a thicker frame cross section only results in a thicker meniscus region, with the central thin region maintaining a sub-100 $\mathrm{nm}$ thickness regardless of frame thickness. If no meniscus is present, frame thickness has no consistent effect on film thickness.

The most pronounced consequence of frame thickness is the range of positions it allows in which a film can form. Some small meniscus region always connects the frame edge to the rest of the liquid crystal film, and as a result is at least as thick as the frame where the two touch. The transition to central film area is not necessarily symmetric on either face of the film-in short, the range of possible film formation planes is equal to the extent of the frame thickness. This is a critical issue for liquid crystal films as targets for a focused laser, since even small inconsistencies in film plane position can result in large deviations in laser-film interaction location with respect to the best laser focus. For fast focusing systems aiming to achieve the highest intensities, the Rayleigh range can be on the order of a few $\mu m$. To prevent radically different intensities of interaction on subsequent film targets, a method must be devised for forming films in a consistent plane, or else the frame thickness must be constrained to be less than the Rayleigh range of the relevant laser system.

One solution to this problem can be found in the use of diamond edges grown within an aperture in a silicon substrate, as shown in Fig. 7.12. These diamond edges can be formed with thicknesses down to 5 or $10 \mu m$, constraining the film formation position to within the 

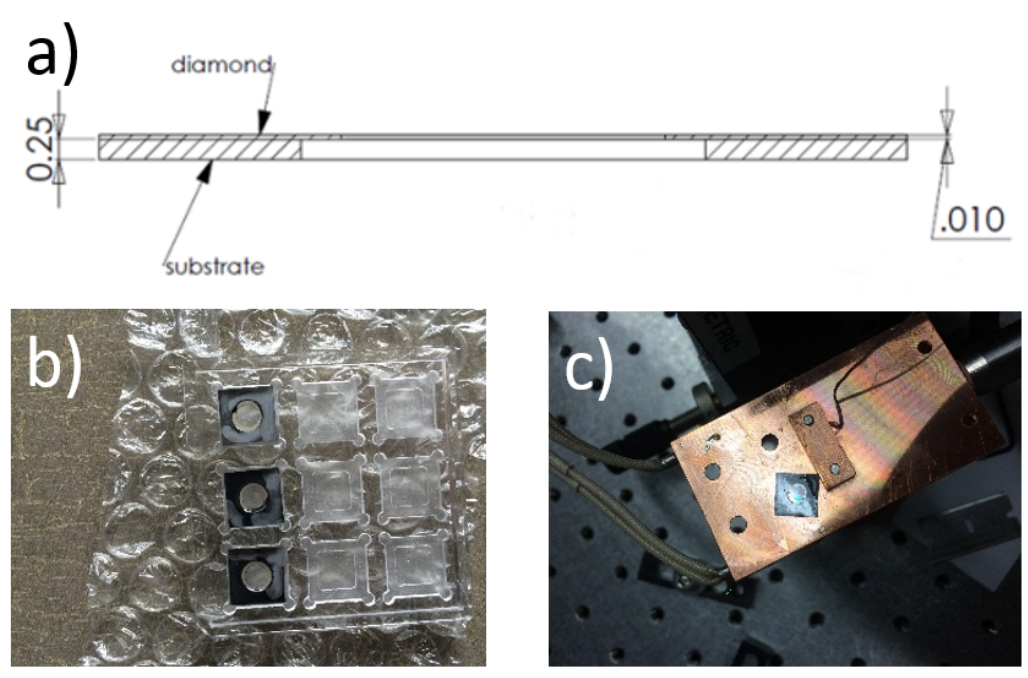

Figure 7.12: Diamond edge frames for precise longitudinal film formation positioning. a) shows a schematic of a $10 \mu \mathrm{m}$ diamond edge grown within the silicon aperture. b) shows the diamond edges as they are bought commercially, and c) indicates how they were implemented on one of the copper frames described above. A liquid crystal film can be seen within the diamond edge aperture on this last image.

$10 \mu m$ Rayleigh range of an $F / 2.2$ system like Scarlet. While these frames can easily be incorporated for film formation because of their superb edge quality, they have the downside of being much more fragile than edges formed on apertures in bulk metal. As a result volume application (typically done with a syringe for precision control) can be dangerous to the diamond edge, and wiping must be done with a gentle material. A standard optical lens tissue is sufficient to form film on these diamond edges, but their fragility and cost make them non-ideal choices for long term high repetition rate film formation. Instead a method of consistently forming films within a thicker frame was developed, which will be described in Chapter 10.

Along these same lines, edge quality is of particular importance to film formation. Edges that have small burrs or are uneven will result in local stresses to the liquid crystal. Small defects can decrease a film's overall lifetime and resilience to vibrations from the air or frame, and can even prevent film formation if the burr is severe. A film with thickness nonuniformity induced by the edge defects is shown in Fig. 7.13.

\subsection{Film target formation}

Once the desired frame parameters have been determined, a number of methods exist for placing this frame at the center of a target chamber for experiments. All target frames need to be mountable to the hardware of the target positioner, which in the case of the Scarlet 


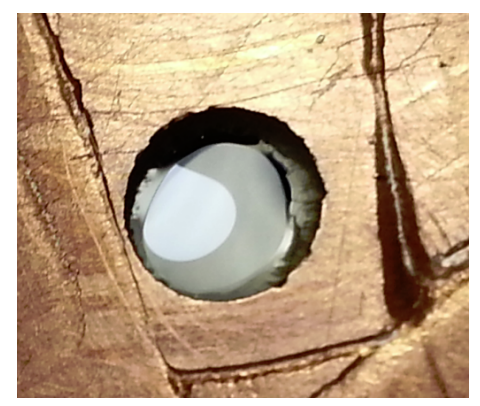

Figure 7.13: Example film formed within a $4 \mathrm{~mm}$ diameter aperture with poor edge quality. Films are more difficult to form in such frames, and have poor flatness and exacerbated thickness nonuniformity.

target chamber is a vertical plate with three linear actuators as well as yaw rotation. A thin copper frame mounted on this target positioner is shown in Fig. 7.14. A thin frame is advantageous at first glance because it allows the Scarlet short pulse beam to pass through an aperture without clipping on a thick frame as it expands past the focus. This aspect is used with unpumped, filtered short pulse light as part of the target alignment procedure, to be detailed in Chapter 8. However, this thin frame was eventually traded for a thicker one that could take advantage of the PID/thermocouple/resistive heater system for precise film thickness control.

It is worth describing the manual, single-shot film formation procedure at this point both to highlight the benefits of the automated techniques developed subsequently and described in later Chapters as well as to underline the robustness of liquid crystal films throughout this process. First, either the thick or thin frame was brought to the desired temperature via a resistively heated pad wrapped around the thin frame or by using the resistive heaters and PID controller for the thick frame. Once the appropriate temperature was reached, a precise volume of $8 \mathrm{CB}$ was applied with the syringe pump. A film was then drawn in the holes of the frame, with the possibility of leaving one aperture free to be used as the handoff alignment target to be detailed in Chapter 9. Drawing within the three apertures proceeded until the desired thickness for the upcoming shots was achieved; films often needed to be redrawn if a non-uniform film was produced or if the thickness was not desired.

Next a $3 \mu \mathrm{m}$ thick aluminum shard was placed in on top of the liquid crystal aperture, to be used as an alignment fiducial. This process is described in detail in Chapter 8 , but is mentioned here to point out the resilience of the liquid crystal film-not only did it survive the placement of shard on top of it, but also the thickness did not change as a result.

The frame would now be transferred from the setup table to the target chamber, where it was mounted on the target positioner. The chamber was then pumped down from air to rough vacuum over the course of a few minutes, then from rough vacuum to $10^{-6}$ Torr 


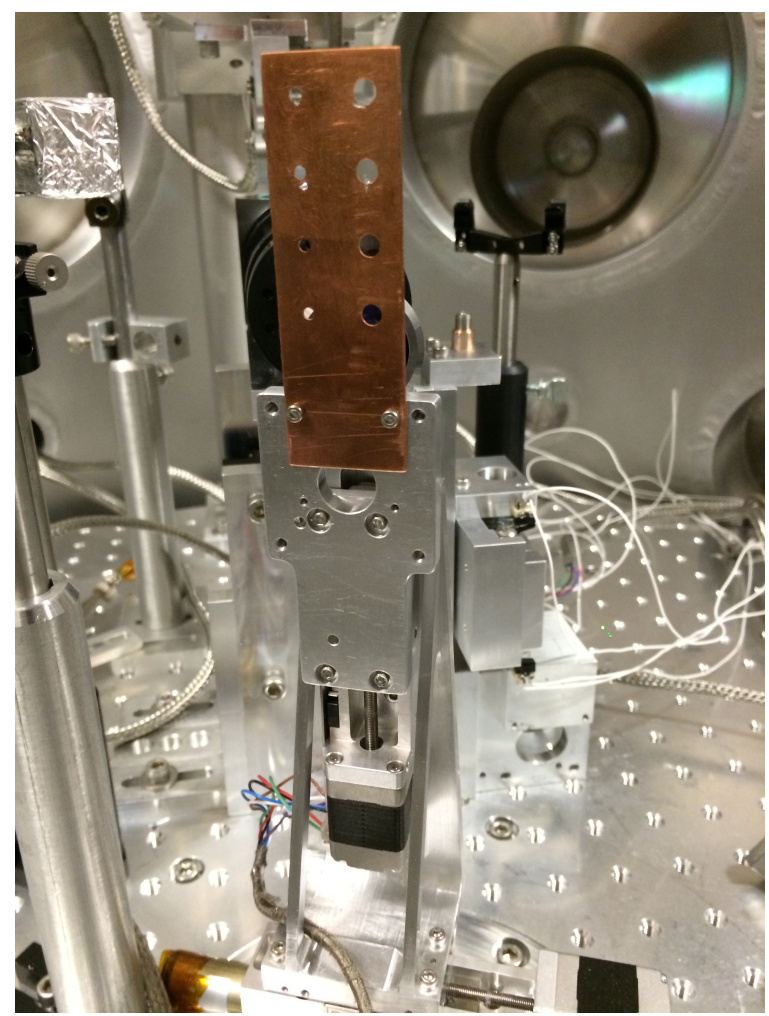

Figure 7.14: Example image of the flat frame set on the target positioner with the Scarlet experimental chamber. This frame holds four possible targets of $4 \mathrm{~mm}$ diameter, and can be heated with a resistive pad outside the chamber to form the desired thickness film. 

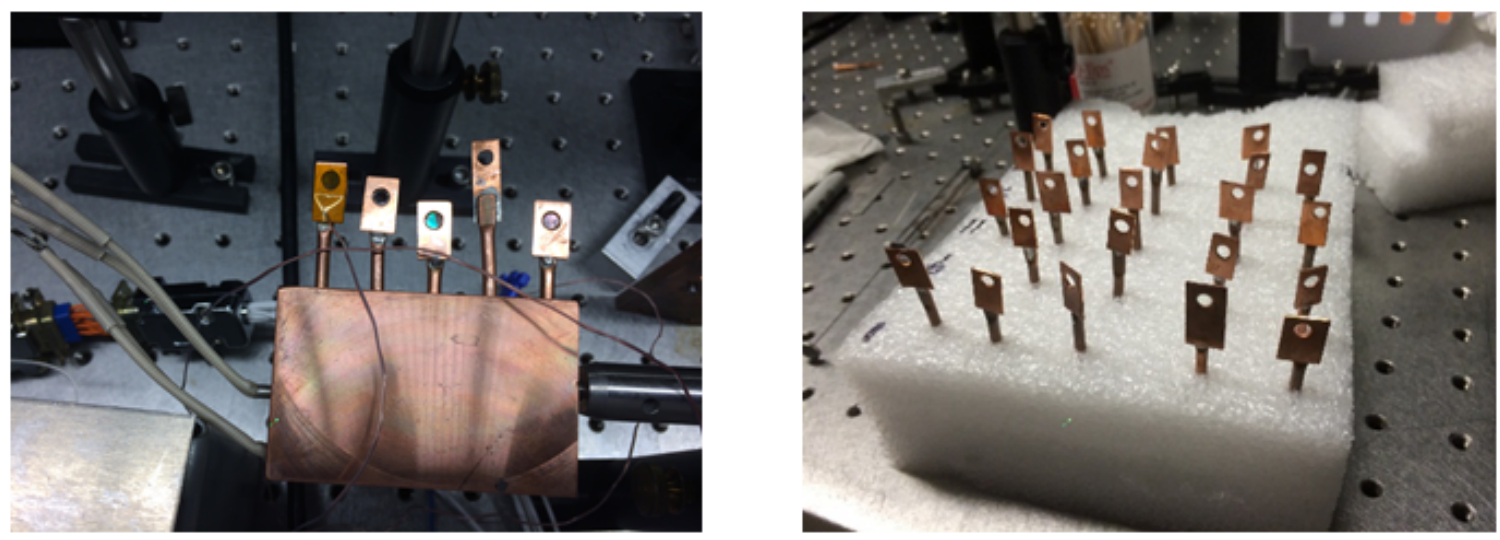

Figure 7.15: Individual copper flags with $4 \mathrm{~mm}$ diameter apertures for film formation prior to shot time. These flags are set in a copper block connected to the resistive heaters and PID temperature control system and can be aligned using a similar block on the target positioner within the chamber. At right are many flag targets with films ranging in thickness from a few-hundred $n m$ to a few $\mu m$. Their resilience allows these films to sit for weeks and longer without changing thickness if left in an environment with modestly regulated temperature.

over the course of tens of minutes. A manual, bellows-sealed angle valve was implemented to open the chamber slowly for initial evacuation, but this was only necessary to prevent the initial rush of air from opening the $50 \mathrm{~mm}$ vacuum tubing connection to the chamber. Even without this gradual opening many films would survive the initial air rush, and with the valve no film ever broke from evacuation. Not only did films survive this entire process, but upon in-vacuo measurement the film thickness was observed to have not changed from the initially drawn value on the setup table.

Though the films were robust enough to survive the formation, evacuation, and alignment procedures with thickness unchanged, the repetitive formation necessary to obtain the desired thickness pushed development of a new target frame. The next iteration is shown in Fig. 7.15: individual copper flag targets with $4 \mathrm{~mm}$ apertures. These could be inserted into a larger copper block (as shown) that was connected to the PID and resistive heaters, allowing precision temperature control. Additionally, a suite of these frames could be made prior to shot time such that films of many thicknesses were available to be inserted as soon as they were desired on the chamber.

\subsection{Chariot and Rail film formation}

Another method of film thickness control can be obtained by forming the film inside a moveable frame. Such a device is shown in Fig. 7.16a, where two copper pieces interlock and can be moved laterally with respect to one another. These pieces can be heated 


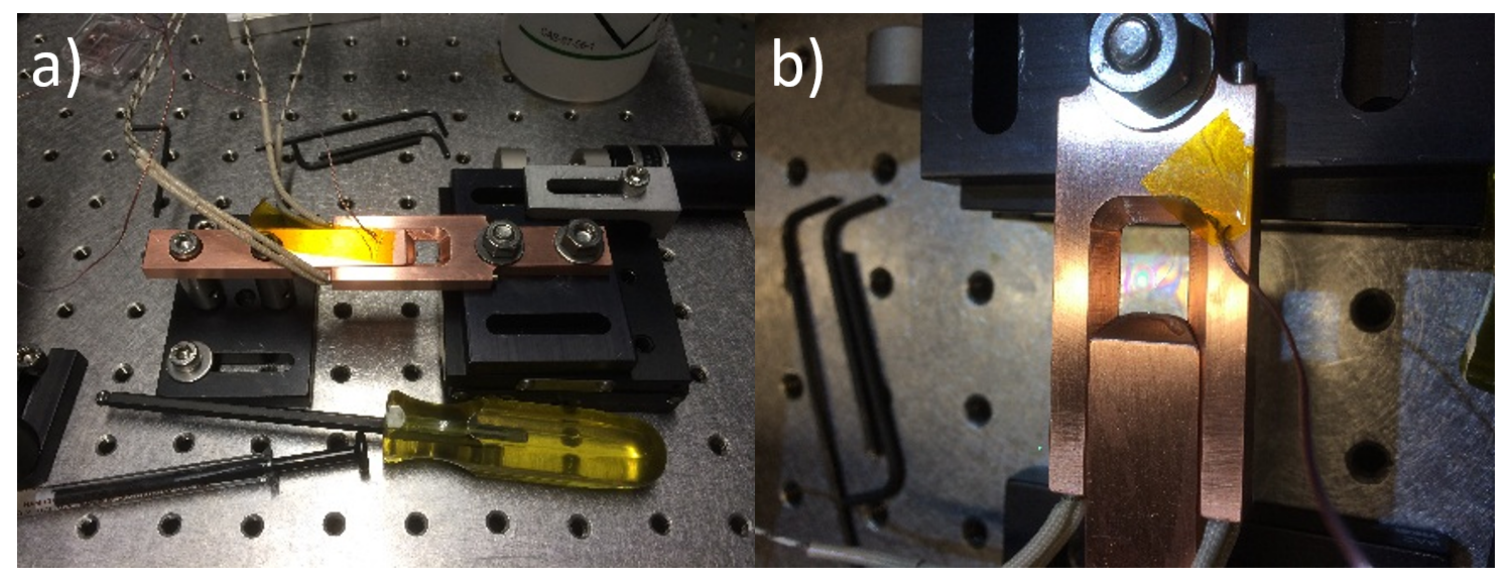

Figure 7.16: The chariot and rail film formation device. Two interlocking copper pieces a) can be heated via resistive cartridge heaters described previously. Liquid crystal applied to the edge between the two pieces will be drawn into a film as they are moved apart. Films several $\mathrm{cm}$ wide are possible with this device. Rapidly stretching a film in this device b) will cause multiple thickness regions of different color to appear.

independently with the same resistive heaters from previous frames. This so-called chariot and rail design and variants thereof have been used previously in fluid dynamics experiments for forming large area films.[127]

Swift changes in the frame area caused the film held within to develop thickness nonuniformity at small nucleation sites away from the frame edges. These thickness variations will eventually work their way throughout the film, typically with the thinnest region ultimately dominating. A picture of an intermediate time in this process is shown in Fig. 7.16b. Slow area modification will cause liquid crystal to enter or leave the meniscus region from the stretched film, resulting in a slow, uniform thickness change. Such a device has been used to make films with areas of several square centimeters, and larger films are possible simply by changing the dimensions of the moveable frame.

The methods described here are sufficient for single film formation. Thickness control even on time scales of tens of minutes is a leap forward for High Energy Density Physics (HEDP) targetry, and unlocks a number of experimental capabilities on its own. Initial experiments with these single-shot films will be described next, and the development of a high repetition rate film formation inserter will follow. 


\section{Chapter 8 \\ Single Shot Experiments on Liquid CRYstal TaRgets}

\subsection{Overview}

Once liquid crystal thickness control was developed, the films needed to be tested as laser targets. As previously discussed, a typical simple High Energy Density Physics (HEDP) target is a small square metallic foil a few $\mu m$ thick and several $\mathrm{mm}$ on each side. Methods for aligning such targets exist, but could not be directly used for the thin, transmissive liquid crystal films. In addition, it was not clear how the films would perform under the standard trials of vacuum preparation and alignment necessary for their use as experimental targets. What follows is a description of the first experiment aligning and firing the Scarlet short pulse laser at liquid crystal film targets, while monitoring the resulting accelerated ion spectrum.

\subsubsection{Film formation}

As described in the previous Chapter, freely suspended films of the liquid crystal 4-cyano4'-octylbiphenyl (8CB) can be controlled if formed in copper frames. Here films were drawn by hand using a razor blade dragged across the copper plates and copper flag target holders shown previously. The methods outlined in the previous Chapter were used to ensure that the films formed were of uniform thickness: though film thickness is affected somewhat by the wiper angle, wiper draw speed, and frame aperture size, the dominant variables are the volume of $8 \mathrm{CB}$ applied and the temperature of the metal frame as the film is being drawn. Volumes as small as $100 n L$ are sufficient to produce a film, which results in extremely low cost per target-the bulk price of the $8 \mathrm{CB}$ used here (from AlphaMicron) was $\$ 15 / \mathrm{mL}$, resulting in a cost per target well under one cent.

Films around $500 \mathrm{~nm}$ and thicker are achievable only with temperature control of the film frame or liquid crystal volume itself. The ideal temperature for thick film formation here was between $27.5^{\circ} \mathrm{C}$ and $28.5^{\circ} \mathrm{C}$, a few degrees below the smectic/nematic phase transition. 
A Proportional Integral Derivative (PID) controller and thermocouple maintained the desired frame temperature to within $0.1{ }^{\circ} \mathrm{C}$ for optimum film formation control. Excess volume draining and island formation inhibit the uniformity of film thickness, but could be minimized if the frame is allowed to cool to room temperature in its horizontal orientation, or entirely eliminated if the film is formed with no meniscus. Under these conditions, films with variable thickness can be readily formed for use in high field physics experiments. The thickness range possible with these procedures is highlighted in Fig. 8.1. The techniques of the previous chapter produce uniform thickness films with minimal meniscus region; although the meniscus region is visible on the thicker films, allowing the frame to cool horizontally ensures islands will not form from this area even once the film is held vertically.
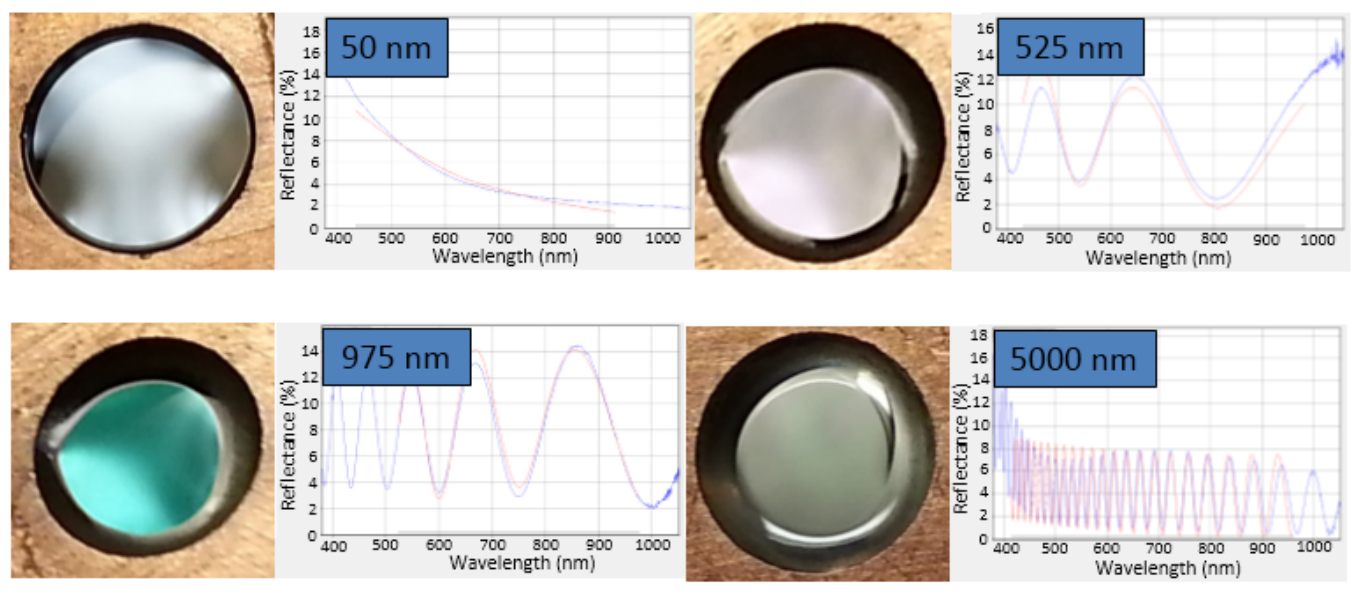

Figure 8.1: Example film thicknesses with measurement from the Filmetrics multi-spectral interferometer demonstrating the thickness range of liquid crystal targets. The plots show reflectance vs. wavelength where the blue curve is the measured reflectance from the film and the red curve is the result of an iterative calculation to match this spectrum. In general, more peaks correspond to a thicker film. The images to the left of each plot show an example film within a copper frame at each of these thicknesses.

It was necessary to have film thickness measurement on the target chamber to see if the chamber evacuation procedure had any effect on film thickness. To this end two relay telescope optics (150 $\mathrm{mm}$ and $300 \mathrm{~mm}$ achromats) were set up inside the chamber and aligned along the front-side target normal direction. The Filmetrics device fiber was placed outside the chamber in front of an uncoated optical window such that it could be positioned to send light into the chamber and through this relay telescope. Achromatic lenses were important for this procedure since the Filmetrics measurement relies on white light focusing near the film to be measured, and any chromatic aberration at the measurement location 
would impede or prevent accurate thickness determination. A shutter was installed within this optical chain so that the Filmetrics device could be protected during a shot.

Of note is the resiliency of liquid crystal films formed in the manner described here. A film drawn with little or no meniscus region will maintain its original thickness regardless of orientation, temperature, surrounding pressure, or gross motion of the frame. As a result the method for using these as intense laser targets is to make the film to the desired thickness using a heated frame, allow the frame to cool back to room temperature, mount the frame within the target chamber, then pump the chamber down to normal vacuum operating levels. Film thickness has been monitored carefully and observed not to fluctuate during these temperature or pressure changes. In fact, liquid crystal films brought down to $10^{-6}$ Torr and not shot were then re-measured once the target chamber had been vented back to atmospheric pressure, at which point their thickness was found to be identical to the original value at formation.

\subsection{Film alignment procedure}

The alignment of the film for these early experiments proved challenging. Typically targets are aligned via some manner of shadowgraphy technique: the center of the target chamber is backlit by an alignment light source, allowing the shadow of the target to be brought into the focal plane of an imaging system and thus defining the center of the target chamber. Bringing the best laser focus also to the focal plane of this imaging system will ensure laser alignment to the target within the limitations of the imaging system used. This sort of technique allows for alignment precision on the tens of $\mu m$ level if properly implemented.

Shadowgraphy within the Scarlet target chamber is typically done using a focal spot alignment camera situated directly behind the target along the incoming laser axis. Light from near Target Chamber Center (TCC) is imaged by an in-situ 10× infinity-corrected microscope objective with a $3.5 \mu \mathrm{m}$ depth of focus over a $0.5 \mathrm{~mm}$ field of view to a camera housed in a small chamber to protect it from vacuum. The camera and objective lens are situated on a vertical translation stage such that they can be moved safely away during a shot.

Alternatives to this shadowgraphy procedure tend to rely on a specular or scattered light reflection from the target to a known location on a camera. Which of these methods works depends on the reflective surface of the target-rough targets are best aligned with scattered reflection techniques, while optically flat targets can be aligned with a specular reflection diagnostic. These methods require somewhat different setups, and so can be cumbersome to swap between if the next experiment target has a different morphology.

The liquid crystal films in their individual copper flags present a problem to all of these alignment techniques. Since the flag is about $1 \mathrm{~mm}$ thick it is difficult to localize one edge 

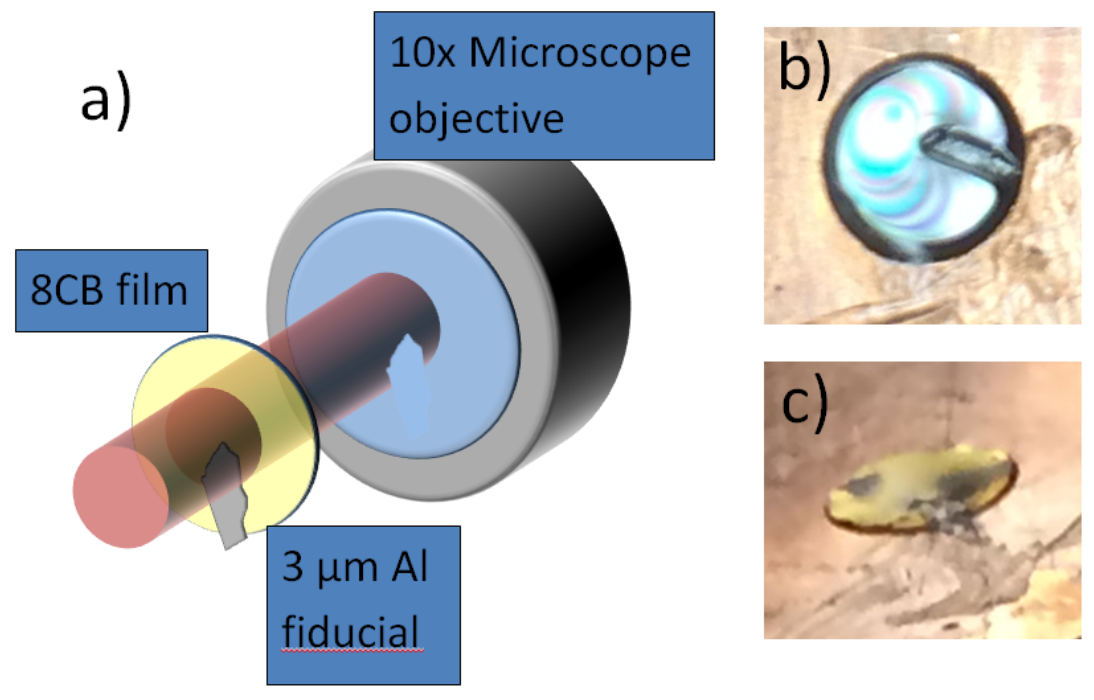

Figure 8.2: a) Cartoon of shadowgraphy alignment onto focal spot camera using $3 \mu \mathrm{m}$ aluminum fiducial floated on a liquid crystal film. b) The fiducial would exacerbate any thickness non-uniformities, but c) careful film formation allowed uniform thickness films to have aluminum fiducials placed within.

of the frame in a shadowgraphy diagnostic, especially down to $10 \mu \mathrm{m}$ accuracy. The films can be made optically flat and so are not easily aligned with a scattered light diagnostic, but their low reflectance makes specular alignment difficult as well.

The solution was to float a small fiducial within the liquid crystal film which could be aligned with standard shadowgraphy. The material chosen was a $3 \mu \mathrm{m}$ thick sliver of aluminum, chosen as thin as possible to minimize its effect on the film surface and so that it could be localized within the $5 \mu \mathrm{m}$ range of the focus alignment camera. It is a testament to the resiliency of liquid crystal films that they are able to have such a fiducial floated on them without changing their thickness. An image of this setup can be seen in Fig. 8.2.

This aluminum fiducial doubled as a way to tell if any films were destroyed during the evacuation or alignment processes, since this would move the fiducial out of its initial position. Before and after images of a film are shown in Fig. 8.3. These images were taken on a large aperture telescope Questar system, which is used for rough target positioning. A close look reveals a slight change in the light signal from the film area, which indicates just how little light liquid crystal films reflect.

\subsection{Experimental results}

The goal of this initial experiment was to evaluate liquid crystal films as ion acceleration targets. For these shots $4 J$ was delivered to the target at a $22.5^{\circ}$ angle of incidence. The 

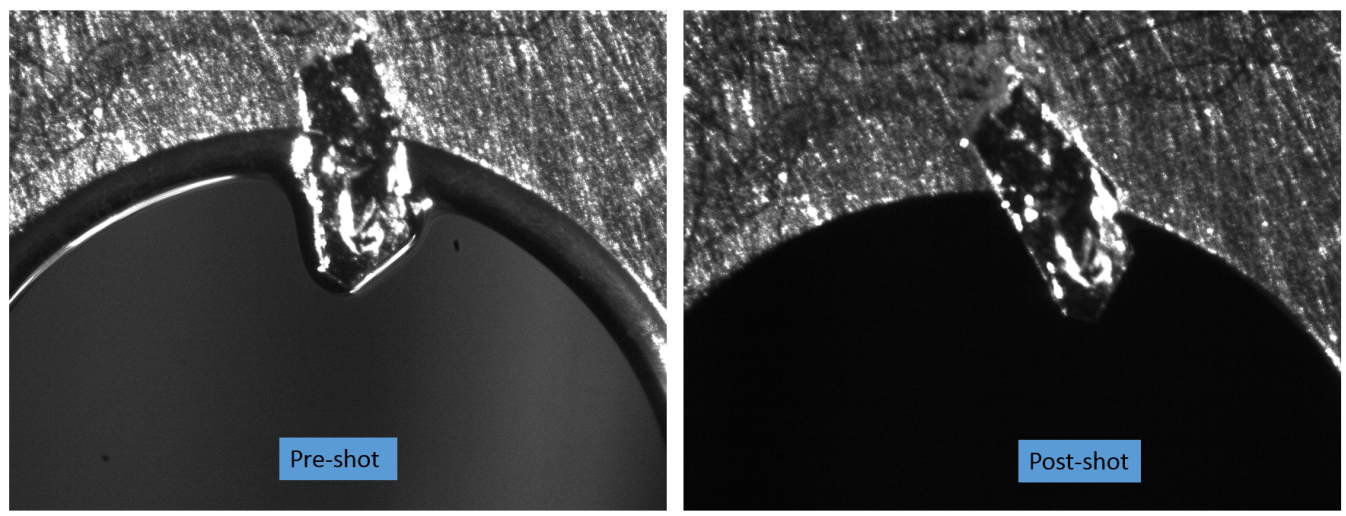

Figure 8.3: $3 \mu \mathrm{m}$ thick aluminum shard floated within edge of $4 \mathrm{~mm}$ liquid crystal frame as viewed from a high field of view telescope Questar system. The left image shows a floated fiducial within a film, while the right image was taken after the laser shot once the film was gone-note the slight change in reflected light within the film area.

chief diagnostic was a Thomson parabola spectrometer[77] located behind target normal to observe Target Normal Sheath-field Acceleration (TNSA) ions. A schematic of the experimental chamber setup is shown in Fig. 8.4. Figure 8.4c shows the shadowgraphy image on the focal spot alignment camera of a fiducial floated in a liquid crystal film. This image could be monitored as the target positioner was moved in $z$ until this feature was sharp, as shown here. The target could then be translated slightly to ensure the laser did not hit the alignment fiducial.

Three ion traces are shown in Fig. 8.5. The first (Fig. 8.5a) is from a $100 \mathrm{~nm} \mathrm{Si}_{3} \mathrm{~N}_{4}$ solid target, while Fig. $8.5 \mathrm{~b}$ and $8.5 \mathrm{c}$ are from a $700 \mathrm{~nm}$ thick and a $160 \mathrm{~nm}$ thick liquid crystal film, respectively. The Thomson parabola spectrometer contains parallel electric and magnetic fields which separate accelerated ions by their charge to mass ratio. Charged particles traveling through these fields will be curved by them, and more energetic particles that spend less time in the fields will be curved less. The result is a parabolic trajectory for each accelerated ion charge species from the target. These ions are incident onto a phosphor imaging plate that can be scanned after the shot to make the images seen here.

Protons have the lowest charge to mass ratio and so are the least curved trace visible in Fig. 8.5 (they are the upper right parabola of each image). The dot in the top left of each image is the neutral point; this is the section of the image plate that has direct line-of-sight of the target chamber center through the Thomson parabola pinhole. As such any radiation or neutral particles streaming from the target will impact this point. The closer an ion trace comes to this point, the more energy it contains.

Maximum proton energies between 5 and $10 \mathrm{MeV}$ were observed for the liquid crystal films, which is consistent with the similar-thickness $\mathrm{Si}_{3} \mathrm{~N}_{4}$ targets. The other traces observed 


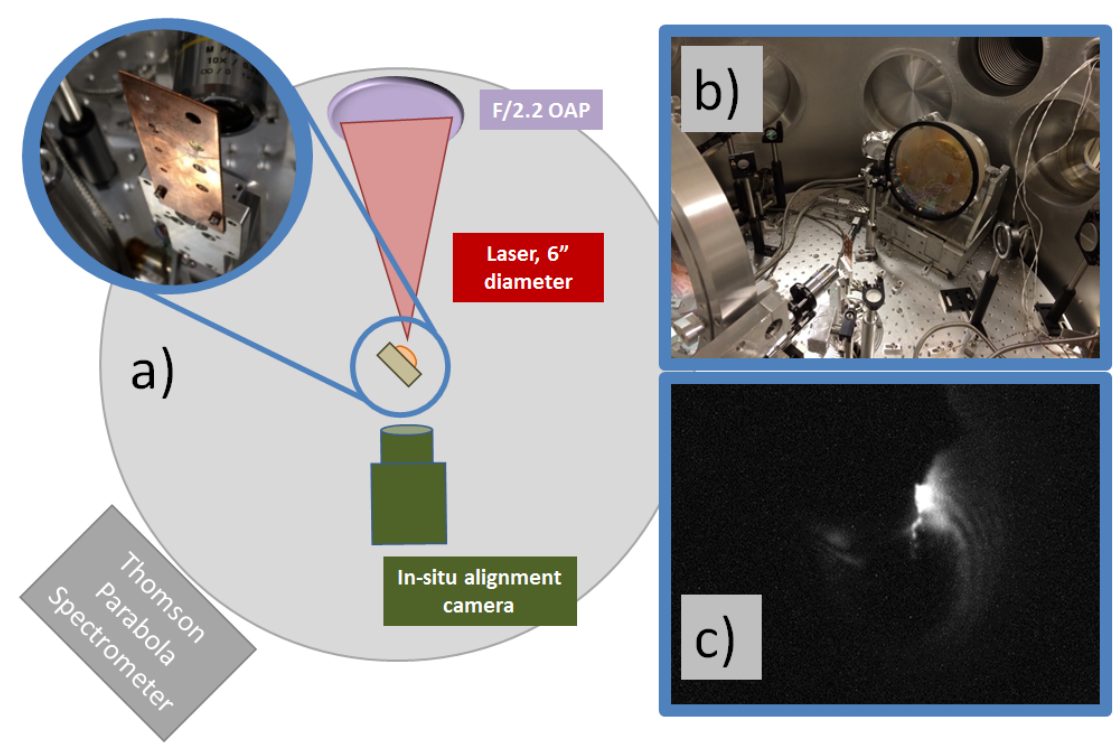

Figure 8.4: a) Schematic of the Scarlet laser main experimental target chamber. A $F / 2.2$ Off-Axis Parabola (OAP) sends the 5.5" diameter beam toward the copper target frame shown in the left inset. An in-vacuum camera situated behind the target along the laser axis is used for alignment, while a Thomson parabola spectrometer collects ion data along the rear-target-normal direction. b) The actual experimental setup with OAP in the background and the target frame and alignment objective in the foreground. c) Focal spot alignment camera shadowgraphy image of alignment light striking the aluminum fiducial floating on a liquid crystal film.

include carbon ions consistent with the known molecular makeup of $8 \mathrm{CB}$, as well as some oxygen ions that are expected from the water vapor contaminants always present in the target chamber. It was possible that any water vapor contamination would be absorbed into the liquid crystal bulk rather than sitting on the surface as it would with a conventional solid target. Since TNSA is strongest for the outer target layers, it is typically hydrocarbon and water vapor contaminants that are accelerated from a target that has been sitting in the chamber for some time. Liquid crystal appears not to absorb these contaminants, however, as their constituents are seen in the ion traces.

This experiment demonstrated freely suspended liquid crystal films as a new kind of target for ultra-intense laser physics experiments. These films are stable in vacuum, inexpensive to produce, and can be varied in thickness on-demand by simple changes in the formation process. Protons accelerated from these thin film targets at various thicknesses measured with a Thomson parabola spectrometer compare favorably to solid target membranes of similar thickness but greatly increased cost. As variable thickness, inexpensive targets they have the capability to unlock a number of experiments that would be difficult or impossible without them. One such experiment is described next. 

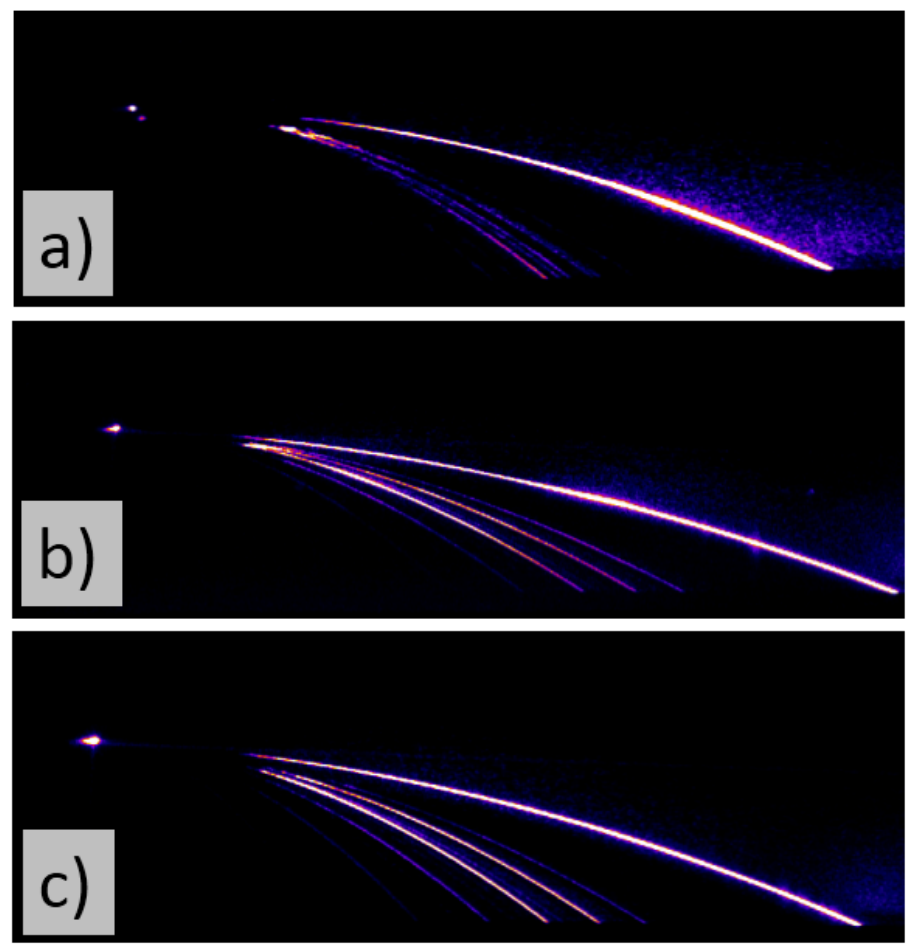

Figure 8.5: Ion acceleration data from the Thomson parabola spectrometer. a) The accelerated ions from a $100 \mathrm{~nm} \mathrm{Si}_{3} \mathrm{~N}_{4}$ solid target are shown as well as b) the traces from a $700 \mathrm{~nm}$ thick liquid crystal film target, and c) from a $160 \mathrm{~nm}$ film. Both liquid crystal ion traces show a strong proton signal with max energy around $10 \mathrm{MeV}$, as well as multiple traces from other ion species, and are comparable to the trace from the similar-thickness solid target. 


\section{Chapter 9 \\ STUdy OF ION ACCELERATION MEChanisms as a FunCtion of TARget Thickness Using Liquid CRYstal Films}

\subsection{Overview}

Initial efforts with the formation of liquid crystal film targets focused on controlling their thickness. As previously described, this allows the opportunity for a systematic study of the onset and optimization of ion acceleration mechanisms whose dominance over the laser matter interaction varies with target thickness. What follows is a detailed description of a first thickness scan using liquid crystal film targets in order to explore the Target Normal Sheath-field Acceleration (TNSA) and Radiation Pressure Acceleration (RPA) ion acceleration mechanisms.

\subsection{Prior literature on ion acceleration thickness scans}

A number of studies on ion acceleration have been done to date, although few with high granularity in target thickness because of the difficulties inherent to making and installing many solid foils of varying thickness. Far more thickness parameter scans have been performed in simulations, with a wide variety of laser parameters.[28, 128, 129] The result usually reported in experiments and simulations is the maximum accelerated proton energy, although in both cases this is often measured for targets shot at normal incidence. As was discussed in Chapter 2, TNSA ions leave along the target normal direction, and RPA leave along the laser axis direction. Ions within a target shot at normal incidence will generally experience some acceleration from both mechanisms-RPA at the front and TNSA at the back side-which can modify the resulting energy distribution.

Different ion energy spectra and maximum energies have been observed for different 
target thicknesses on different laser systems.[24, 28, 128, 129] This appears to be a function at least in part of the following: laser energy, pulse duration, contrast, and target makeup. Optimizing a desired ion acceleration mechanism requires an exploratory scan on the laser facility to be used, perhaps to be redone if the laser parameters ever change appreciably. Such a scan has not previously been done over the entire range thicknesses spanned by the TNSA and RPA mechanisms.

\subsection{Liquid crystal thickness scan setup}

The goal of this experiment was to perform multiple shots at a number of thicknesses of liquid crystal film targets between $100 \mathrm{~nm}$ and $2 \mu \mathrm{m}$ and to monitor the accelerated ions on each shot. The liquid film targets were made in $4 \mathrm{~mm}$ diameter apertures in copper flags as in the previous experiment. They were made on a setup bench where the frame temperature and volume deposition could be carefully controlled to achieve the desired thickness. Once formed the films were moved over to the target chamber and installed into the target positioner before evacuation of the chamber. Films went through final alignment to target chamber center before a last thickness measurement was made to ensure no change from the installation and alignment procedure.

The alignment difficulties for liquid crystals were described in the previous Chapter. To avoid these issues, a confocal positioning device was developed to align liquid crystal films. The Confocal High Intensity Positioner (CHIP) (Fig. 9.1) operates on the principle of confocal microscopy: alignment light enters the device via a beamsplitter and is subsequently focused by a $10 \times$ objective lens onto the target at normal incidence. The reflection from the target is then collimated by the objective lens, passed this time through the beamsplitter and into another lens that focuses the light onto a small pinhole-in this case a $10 \mu \mathrm{m}$ core single-mode fiber. If the target is not at the correct $z$ location this alignment light will not be imaged correctly through the system resulting in a spot size larger than the $10 \mu \mathrm{m}$ fiber input and therefore a loss of detected light on the other side of the fiber. This $z$ position measurement is quite accurate due to the decreased Rayleigh range of the $532 \mathrm{~nm}$ alignment light compared to that of the $800 \mathrm{~nm}$ short pulse, as well as the fact that any $z$ error is doubled for the alignment light as it reflects from the target. In this way accuracy of $\pm 1 \mu \mathrm{m}$ is possible with the CHIP, well within the $5 \mu \mathrm{m}$ Rayleigh range of the short pulse focus.

This CHIP system aligns liquid crystal films well, removing the necessity for the alignment fiducial from the previous experiment. As an added benefit the device also aligns any other target to the same level of precision regardless of surface morphology, a fact that cannot be said about conventional specular or scatter alignment diagnostics.

It is worth describing the alignment procedure necessary for using the CHIP as these 
details will be important for the analysis of the presented experimental results. The goal is to bring the best laser focus and the target to the same position-this is done by swapping the definition of Target Chamber Center (TCC) between a number of devices.

First, a focal spot alignment camera images the light from somewhere near the focus of the Off-Axis Parabola (OAP). This diagnostic consists of a cage-mounted Mitutoyo apochromatic infinity-corrected microscope objective $(10 \times$, near $F / 2)$ placed its working distance $(2.7 \mathrm{~cm})$ from the nominal center of the chamber. After this is the corresponding tube lens $(f=200 \mathrm{~mm}$ ) followed by a small aluminum box that houses a 12 bit GigE camera. This allows the camera to stay at atmospheric pressure thereby protecting the main target chamber from camera outgassing and preventing the camera from overheating in vacuum. Due to limited space directly behind the target, the focal spot camera cage system has a turning mirror to send the light vertically down to the camera. The entire device is placed on a vertical translation stage so that it can be lowered to safety during a shot.

This focal spot camera observes the short pulse laser focus in a partially amplified, heavily filtered state. Optimizations of the OAP are performed to achieve the best focal quality, at which point this focal spot alignment camera defines TCC.

Next, a "hand-off" target is moved into a position near TCC. This target consists of a glass microscope slide $100 \mu \mathrm{m}$ thick covered on one side with copper nanoparticles. The nanoparticles are deposited by sonicating a few $\mathrm{mg}$ in $100 \mathrm{~mL}$ of deionized water to achieve even distribution and then placing a droplet of this mixture onto the handoff slide. After the water evaporates, a layer of $\mu m$-sized groups of nanoparticles remains on the front side of the handoff target. These nanoparticles serve the dual purpose of aligning a target to the focal spot alignment camera and subsequently to the CHIP as well, hence the name.

These handoff target nanoparticles can be illuminated with wide aperture alignment light made to travel along the short pulse laser path which casts a shadow visible on the focal spot alignment camera. Moving the handoff target until these shadows are in focus shifts the definition of TCC to the front surface of the handoff target.

The CHIP is then moved into place such that its alignment beam focuses near the front side of the handoff target. First, the CHIP alignment light can be positioned laterally such that it strikes the same location on the focal spot alignment camera as the short pulse focus did previously- this ensures the positioner is being aligned to the location where the laser will hit. Next, strong reflection from the nanoparticle groups will cause high signal through the CHIP pinhole when the handoff target is in the best focal plane. Once this signal is maximized, the CHIP now defines TCC-this time to within $\pm 1 \mu \mathrm{m}$. This definition of TCC matches the location of the best laser focus to within the Rayleigh range of the focal spot alignment camera objective, which is about $10 \mu m$ on the $F / 2$ Scarlet target chamber.

After this procedure is done once, any subsequent targets are moved into place such 

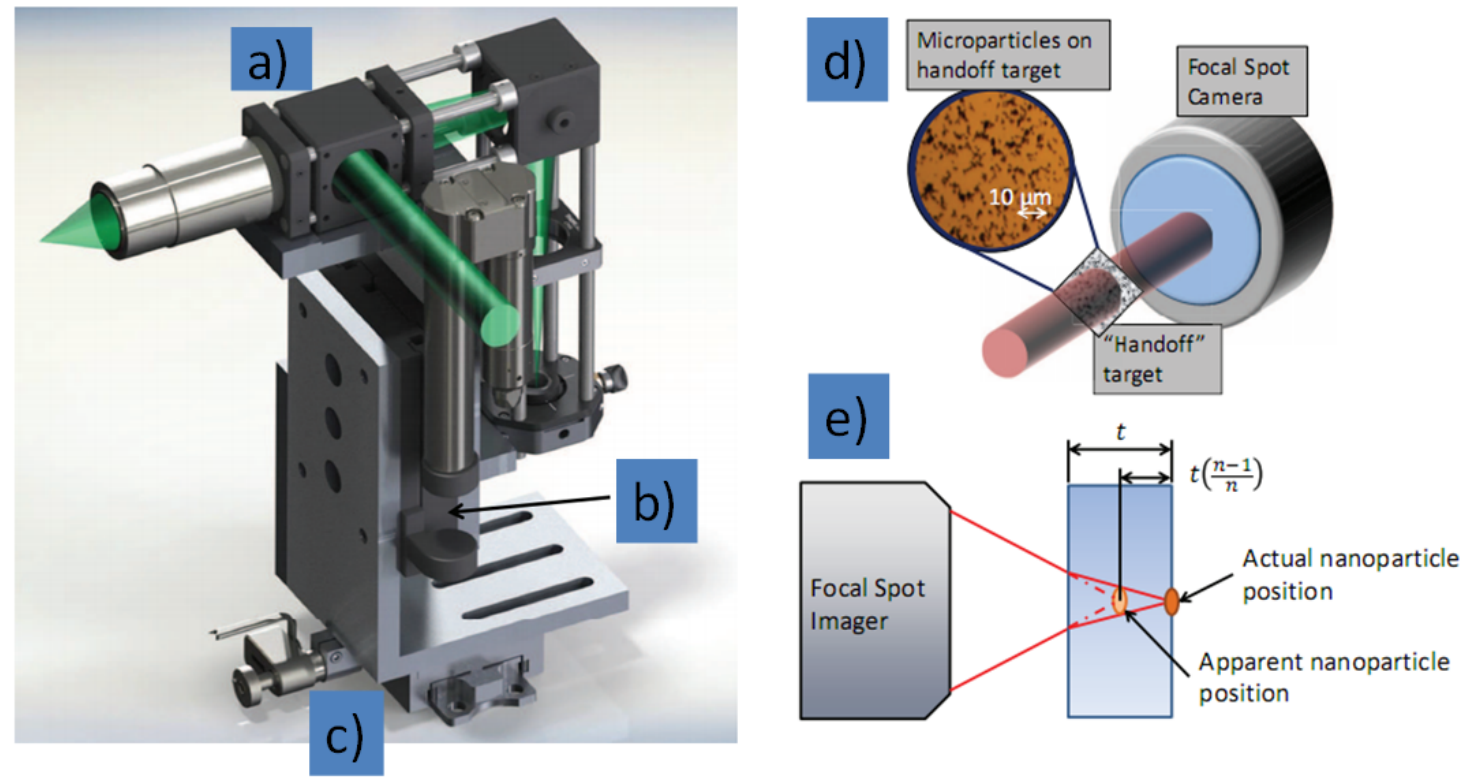

Figure 9.1: a) The CHIP is designed in a cage-mount system for compactness and rigidity to ensure its precision. b) A vertical translation stage allows the input optics to be lowered to safety during a laser shot. c) A motorized stage allows the CHIP to be finely aligned along the front-side target normal direction. d) Cartoon of the target handoff procedure including shadowgraphy of the copper nanoparticle coated slide. e) Diagram of the mismatch in actual and apparent focal plane of the focal spot alignment camera due to light traveling through the handoff target.

that maximum signal is achieved on the CHIP. Critically, this is done by moving the target positioner and not the CHIP, since the latter defines TCC. Like the focal spot camera, the CHIP can be moved vertically down out of the target interaction plane during a shot to protect its optics-since the CHIP is brought back to an identical position (on the $\mu \mathrm{m}$ level) when raised, this handoff procedure needs to be done only once per vacuum chamber cycle.

The layout for the experiment is shown in Fig. 9.2. The primary alignment elements are the focal spot alignment camera directly behind the target and along the laser axis as well as the CHIP device positioned to observe the front side target normal. Both of these devices are held on vertical translation stages which allow them to be lowered for their protection during a shot. Additionally, the Filmetrics fiber input telescope was still present for this experimental run allowing film thickness measurement at any time leading up to a shot.

The primary diagnostics for the experiment were a Thomson parabola spectrometer located along the rear target normal direction and a pair of radiochromic film (RCF) packs located along the target normal and laser axis directions. These diagnostics were intended 


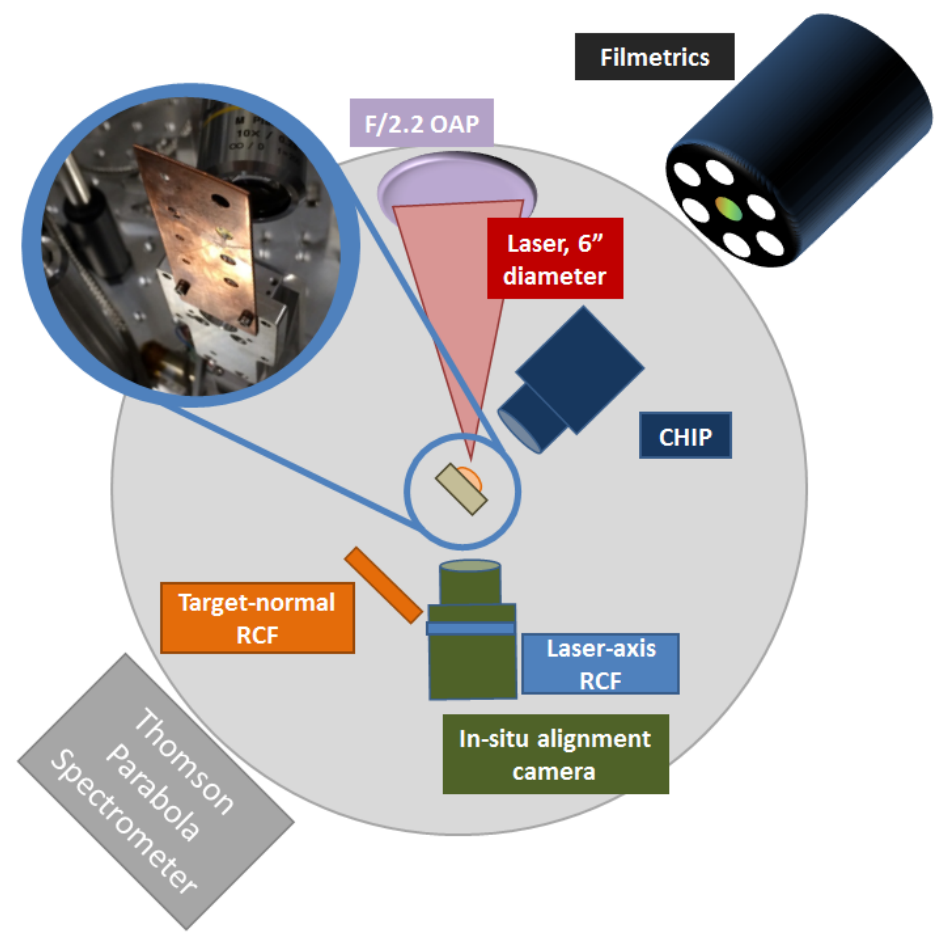

Figure 9.2: Cartoon of experimental chamber for thickness scan run. Of note are the CHIP and focal spot camera for alignment, the Filmetrics for target thickness measurement, and the Thomson parabola and two RCF stacks for accelerated ion measurement.

to provide spatial information on the accelerated ions as well as to distinguish between acceleration occurring along the rear target normal direction-like TNSA, which should dominate around $1 \mu \mathrm{m}$ target thicknesses-and a mechanism like RPA, which turns on at target thicknesses below $1 \mu \mathrm{m}$ and sends ions along the laser axis direction. The liquid crystal films were shot at $22.5^{\circ}$ angle of incidence so the ions accelerated from these two mechanisms could be distinguished. The RCF pack along the target normal direction had a $4 \mathrm{~mm}$ diameter clearance hole to allow ions directly leaving the target normal surface to reach the pinhole for the Thomson parabola. The layers and filtering of the RCF stacks were identical, and chosen to reveal protons in a range from $3 \mathrm{MeV}$ to $>13 \mathrm{MeV}$.

The RCF stacks consisted of sheets of MD-V2, MD-V3, and EBT type films, with 25 $\mu m$ of $\mathrm{Al}$ between sheets 4 and 5 and $25 \mu m$ of $\mathrm{Cu}$ between sheets 5 and 6 . This film type selection and filtering location set the energy of protons that would be deposited into each film; these values are listed in Fig. 9.3. Images of the scanned RCF data will be laid out in the order indicated Fig. 9.3, with the lowest energy sheet in the upper left and the highest energy sheet in the bottom right. The black numbers in the lower right of each sheet will also be on any subsequent data images. Figure 9.3 also shows a calculation of the energy 
a)

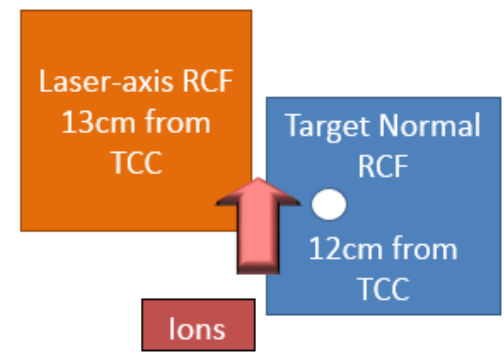

b)

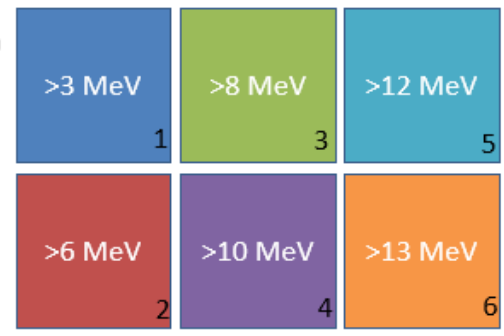

c)
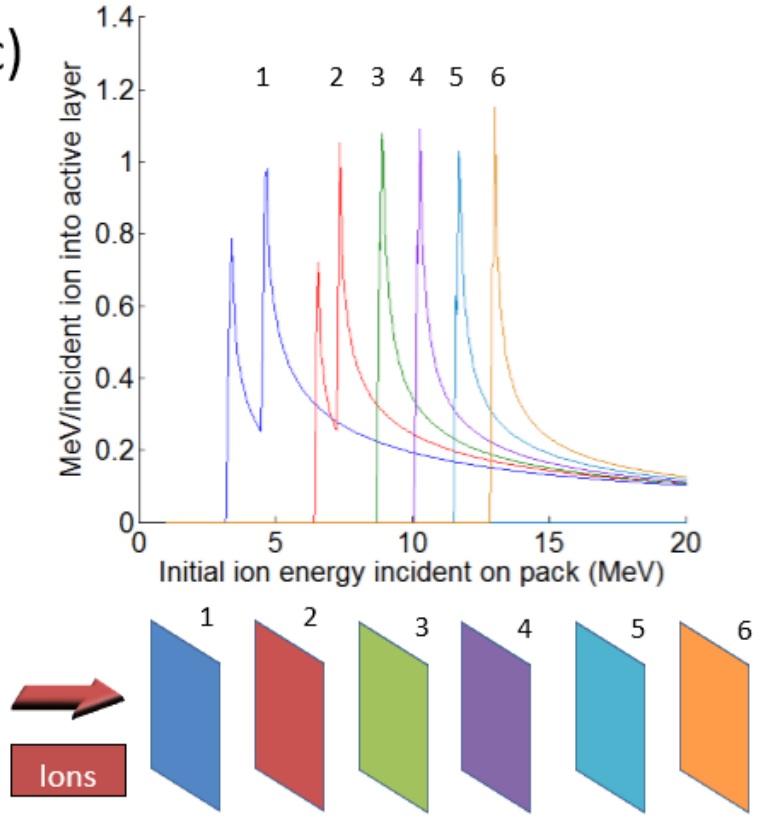

Figure 9.3: a) Spatial orientation of the two RCF stacks. The one on the right (looking along the laser $k$-vector) had a $4 \mathrm{~mm}$ hole punched to allow ions to travel directly to the Thomson parabola beyond. b) Each RCF stack was comprised of six pieces of film with appropriate filtering to observe proton energies indicated here in white. The layout of the films shown here will be replicated for all subsequent RCF images presented. The black numbers in the lower right of each layer will be visible as well. c) Graph showing the deposited energy into each layer of the RCF stack. Higher energy protons travel through the initial stacks with relatively little dose and deposit most of their energy within a subsequent stack once their speed has been reduced by collisions in the previous layers.

how much dose a will be deposited into the various layers of a stack based on the initial incident energy of that particle. Protons stopping in the RCF pack exhibit a Bragg energy deposition curve, where they are slowed down by collisions until they suddenly deposit the bulk of their energy at one location (indicated by the sharp peaks in the graph).

\subsection{Data}

The primary result from this experimental run is shown in Fig. 9.4. Each point represents, for a single shot, the maximum proton energy along the target normal direction as measured by the Thomson parabola spectrometer. Here most of the data points are liquid crystal films,

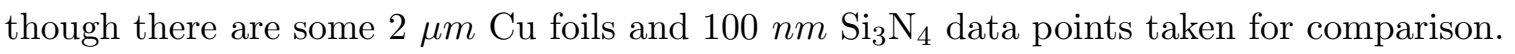
A clear peak in this data can be seen in the 600-900 $\mathrm{nm}$ target thickness range, with the highest proton energy of $24 \mathrm{MeV}$ recorded for targets near $600 \mathrm{~nm}$ thick. 


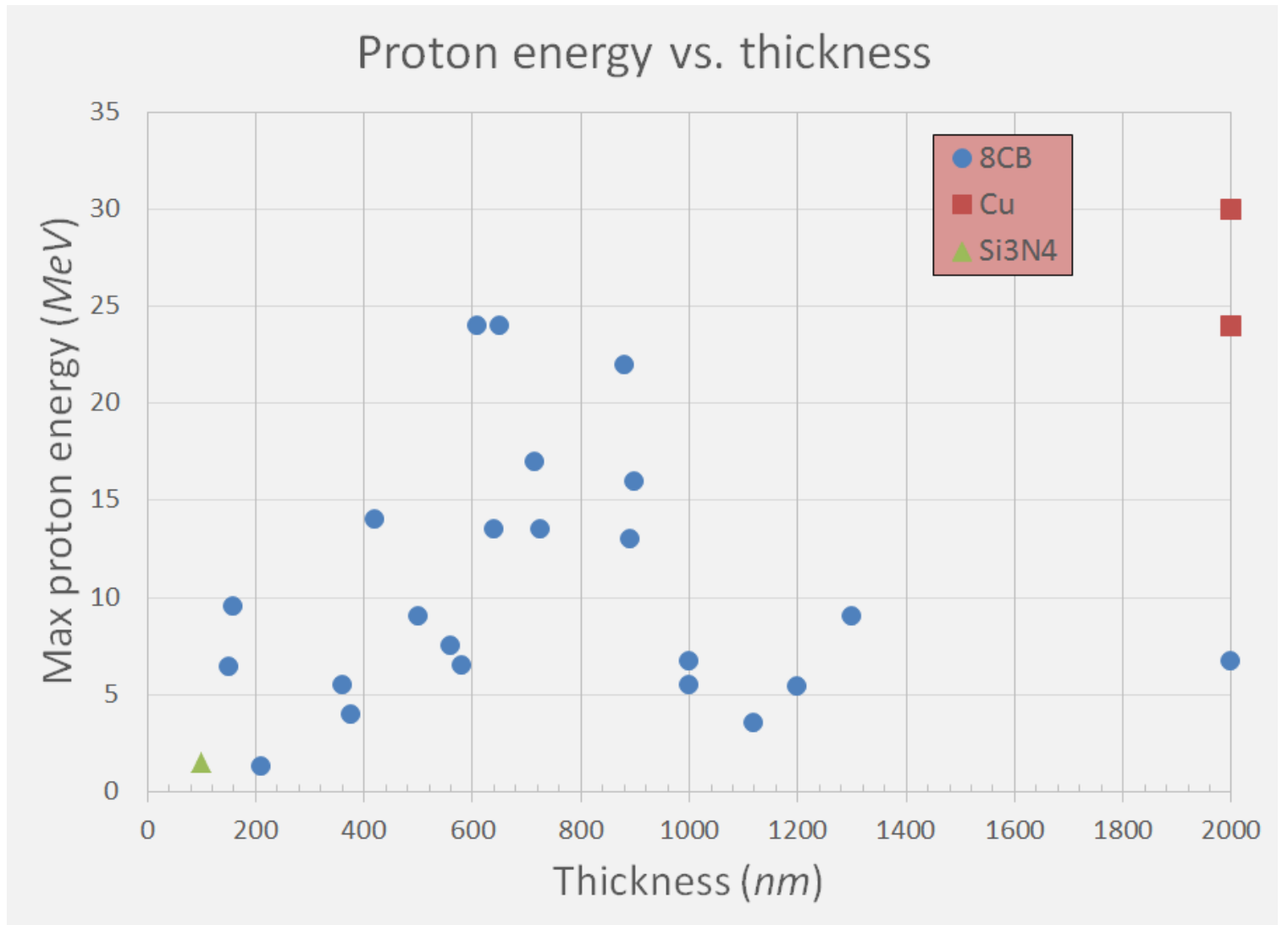

Figure 9.4: Maximum proton energies recorded along the target normal direction by the Thomson parabola spectrometer for various thicknesses of liquid crystal films. Also plotted are two $2 \mu \mathrm{m} \mathrm{Cu}$ foils and one $\mathrm{Si}_{3} \mathrm{~N}_{4} 100 \mathrm{~nm}$ membrane for comparison. There is a broad peak observed in the 600-900 $\mathrm{nm}$ range, suggesting an optimization of TNSA here. 
This value is lower than expected for the optimization of target normal directed protons. Additionally, strong signal along the laser axis direction was observed for very few shots. These results suggested that inconsistent or insufficient intensity was being delivered to the target, either from a strong pre-pulse (not thought to be an issue on Scarlet) or from improper alignment of focus.

The latter was later discovered to be the case, resulting from the nature of the CHIP alignment at the time. The device as described above relies on alignment light reflecting from the front surface of the handoff target, which was a $100 \mu \mathrm{m}$ glass slide covered on one side with nanoparticles. The effects of refraction through this slide were not considered originally-essentially any image viewed through this slide (as it is on the focal spot alignment camera) will be shifted in apparent $z$ position by $1 / 3$ the thickness of the slide. In the present case that amounts to $\sim 30 \mu \mathrm{m}$ of $z$ misalignment on each shot. This is shown in Fig. 9.1e. As a result, this experimental data amounts to a defocused run: the spot size on target was larger than best laser focus, but the CHIP alignment ensured a consistent positioning with $\pm 1 \mu \mathrm{m}$ around that $30 \mu \mathrm{m}$ offset on each shot.

The resulting laser intensity from the $10 \mathrm{~J}$ energy in $40 \mathrm{fs}$ pulse duration was $5 \times$ $10^{19} \mathrm{~W} / \mathrm{cm}^{2}$. This is on the threshold of the onset of RPA, which suggests why it was not observed on most of the shots. Additionally, variations in the laser mode are more likely at defocused spot sizes. Despite this, the data presented here is an important investigation into the optimization of the TNSA mechanism on the Scarlet laser, as this is the only run to date where identical conditions (laser parameters, target material, etc.) have been used to do a thickness scan searching to optimize ion acceleration over so large a range of thicknesses. This experiment was only possible because of the versatility and easy of use of liquid crystal films.

Despite the overall low laser-axis signal, a few shots did show particularly good laser-axis signal. The data for one of these shots is shown in Fig. 9.5. RCF is initially a transparent bluish hue, but turns opaque dark blue upon irradiation by ions. The thickness and type of material in each layer is known and determines what energy of proton will deposit optimally into the film. Subsequent layers of the stack will only see higher energy protons which were able to penetrate previous layers but were slowed down enough in the process for their Bragg peak to occur within the next layer.

For the shot under consideration a higher proton dose is recorded for the laser-axis RCF stack than for the target-normal stack. The laser-axis stack had on its front surface a thin piece of Columbia Resin 39 (CR39) with $1 \mathrm{~mm}$ holes drilled through. This CR39 also absorbs incoming photons, serving to decrease the energy of any particles that pass through it. For these images it serves as an additional filter-in essence, the fact that the CR39 is clearly visible, both its holes and rectangular outline, indicates a strong proton signal. Note in particular the dark blue signal in layers 3 and 4 of the laser axis stack, which are more 
$640 \mathrm{~nm} \mathrm{8CB}$-laser axis
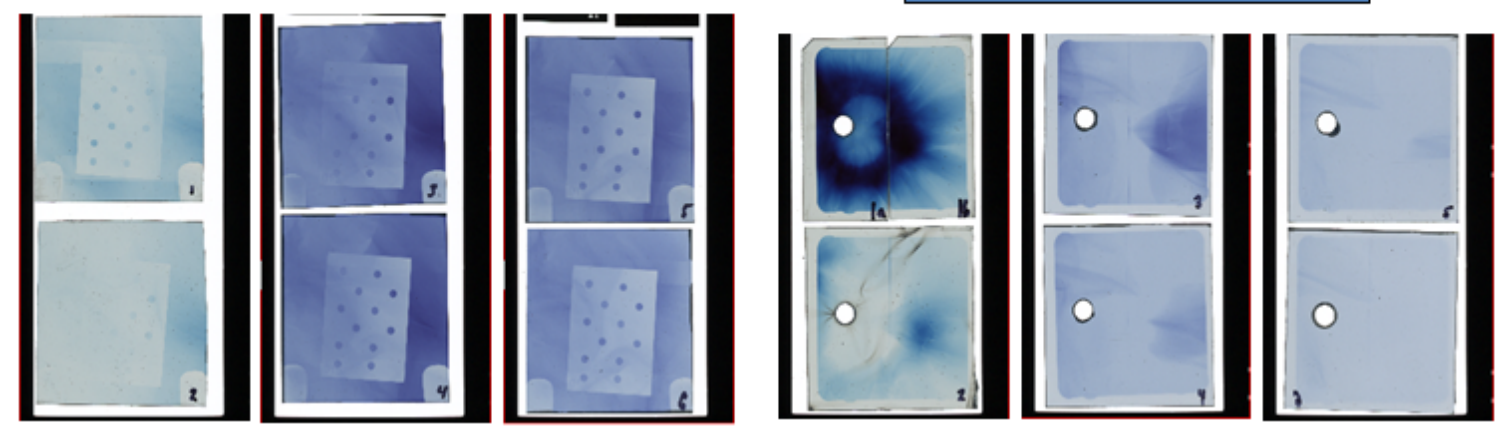

Figure 9.5: $\mathrm{RCF}$ data from a $640 \mathrm{~nm}$ 4-cyano-4'-octylbiphenyl (8CB) film. The laseraxis stack has more dose (i.e. darker blue films) than the target normal sheet, which clearly indicates a non-TNSA acceleration mechanism. The shadow of a rectangular piece of CR39 with holes drilled in it can be seen in the laser-axis sheets as well-this essentially acted as an extra filter, raising the energy of protons necessary to reach each of these sheets.

opaque than the corresponding images for the target-normal direction.

Additionally, this strong signal is observed on layers 3 and 4 of the laser-axis stack but not on layers 1 and 2. This rules out the exponential spectrum indicative of TNSA (which would have the strongest signal on the earliest layers), but also is not as quasimonoenergetic as has been seen before on RPA simulations.[24, 28] This is perhaps chiefly a result of the near-threshold intensity on this shot, but also stems from other factors: the use of linear polarization at non-normal incidence hinders the balance between ponderomotive pressure and electrostatic pressure set up as the laser pulse is reflecting. The result is less efficient laser energy conversion into the accelerated ions, which serves to broaden any peaks in the energy spectrum. Additionally, the presence of the TNSA field at the rear of the target will also have an effect on the ion accelerated from the front side, causing the distribution to broaden. In short this spectrum is not unexpected due to the nature of this experimental setup: while other work seeks to optimize this monoenergetic peak, the goal of this experiment was to differentiate between the two effects, which necessitates the off-normal incidence and its inherent spectral broadening.

A quantitative analysis of the laser axis spectrum on this shot can be seen in Fig. 9.6. Here the corresponding layers of RCF from each direction have been digitally stitched together, and then the recorded dose has been integrated over concentric disks as shown in Fig. 9.6a. The result is a measure of the deposited energy density as a function of angle away from the target normal direction. The blue and green traces in Fig. 9.6b are from layers 1 and 2, which saw much higher dosage than any subsequent film, and most of it near the target normal direction. This is the ring of dark blue visible in the first target normal 

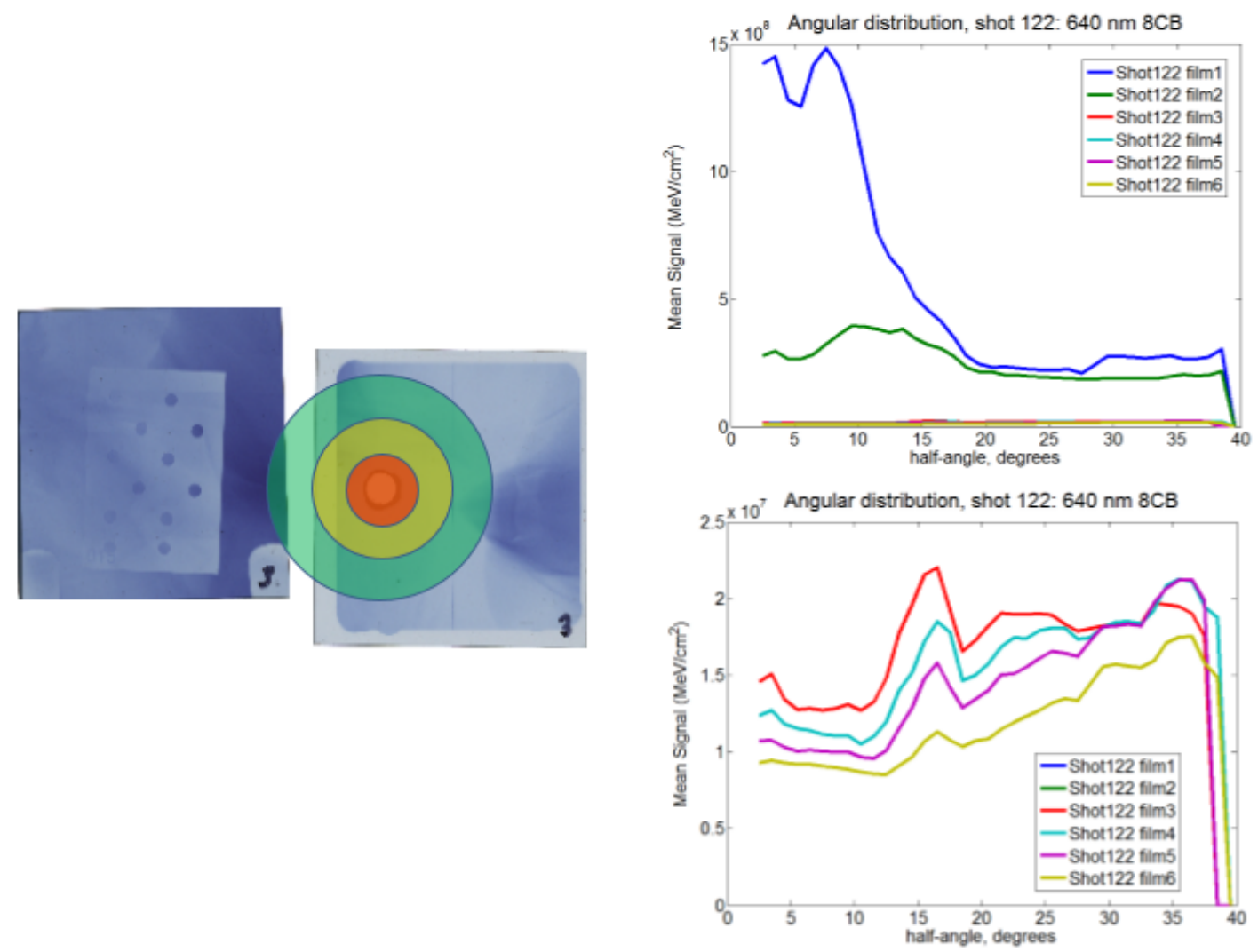

Figure 9.6: a) Spatial location of the two RCF packs with an overlay demonstrating the radial integration process for angular dose signal collection. Zero degrees is set to be the target normal direction. b) Graph of deposited energy density as a function of angle away from the target normal direction for all six film stacks on a shot of $640 \mathrm{~nm} 8 \mathrm{CB}$. c) Angular data from layers 3-6, where an increasing dose as a function of angle appears. This behavior is indicative of an ion acceleration mechanism other than TNSA.

layer in Fig. 9.5.

Figure 9.6c shows the signal from all layers after the first two, in which an increasing signal as a function of angle away from target normal can be seen. This is the case despite the CR39 layer acting as an addition filter removing some of the laser-axis-directed proton signal from these films.

This strong laser axis ion data was taken for a $640 \mathrm{~nm}$ film but was not replicated to a lesser degree on other shots near $600 \mathrm{~nm}$. For example, Fig. 9.7 shows a $715 \mathrm{~nm}$ liquid crystal shot with a more typical signal falloff away from target normal, as compared to the shown $610 \mathrm{~nm}$ angular data which exhibits a different (though still decreasing) shape. This $600 \mathrm{~nm}$ thickness is well above the range thought to be optimal for a mechanism like RPA, and so it's possible a different ion acceleration mechanism was acting in this experiment. 


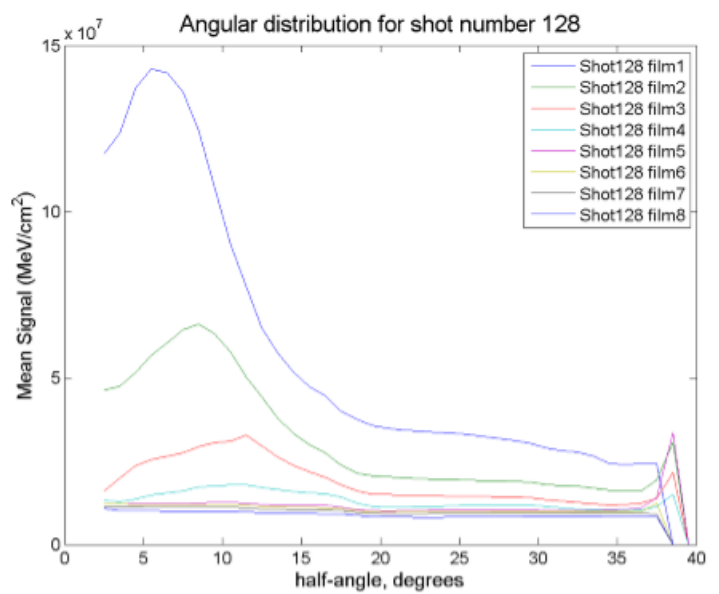

Shot 128: $715 \mathrm{~nm} \mathrm{8CB}$

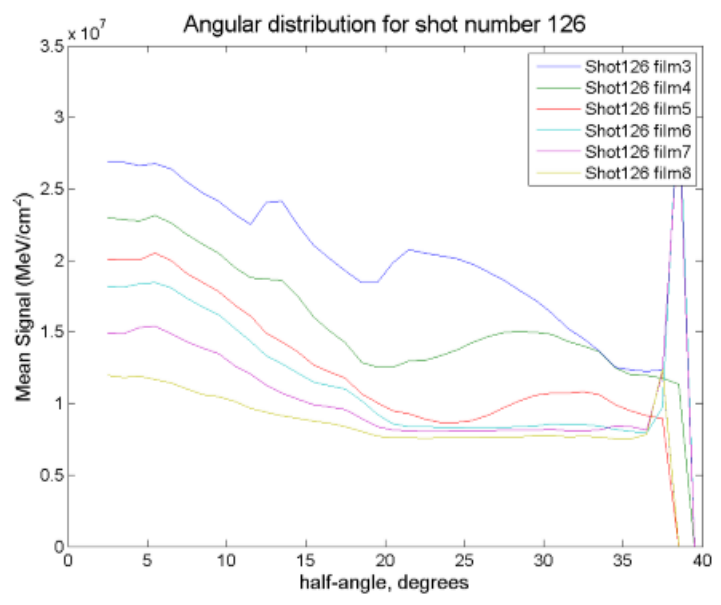

Shot 126: $610 \mathrm{~nm} \mathrm{8CB}$

Figure 9.7: Angular dose data from two different liquid crystal shots. The left chart is of a $715 \mathrm{~nm}$ film that exhibits a sharp decline in signal away from the target normal direction, indicative dominant TNSA acceleration. The data on the right is from a $610 \mathrm{~nm}$ film with a slighter slope, suggesting a contribution from a non-TNSA mechanism at this thickness.

Further evidence of this is discussed in the next section.

\subsection{Discussion}

Other features of the data set unexpected during the planning phase of the experiment are worth some discussion. Of note is the strong ring-shaped proton signal visible on the low energy target-normal RCF layers. Examples of these rings are visible in Fig. 9.8. This annulus pattern was visible on almost every liquid crystal film shot, although the strength or size of the signal did not correlate with on-shot laser intensity or target thickness. It was clearly not present on the copper and silica nitride shots taken as part of this experimental campaign, as can be seen in Fig. 9.8. The annulus also did not appear to be a strong function of pulse width, as $100 \mathrm{fs}$ shots on liquid crystal films still resulted in this feature.

This annular feature could result simply from a decreased intensity at the wings of the incoming laser spot size, which would produce a low energy ring of protons. In this case higher energy protons should come from the central regions of the Gaussian focal spot, which would show up as darker spots within the annular region on subsequent layers of the RCF stack. This effect is enhanced by the decrease in divergence angle for higher energy protons, [130] such that high energy protons emerge in a smaller angle. Protons accelerated from the transition region of the Gaussian focus (i.e. where the intensity gradient increases sharply from wing to pulse peak) will be pushed out to larger divergence angles and thus produce a sharp edge in the annular shape.[130] 

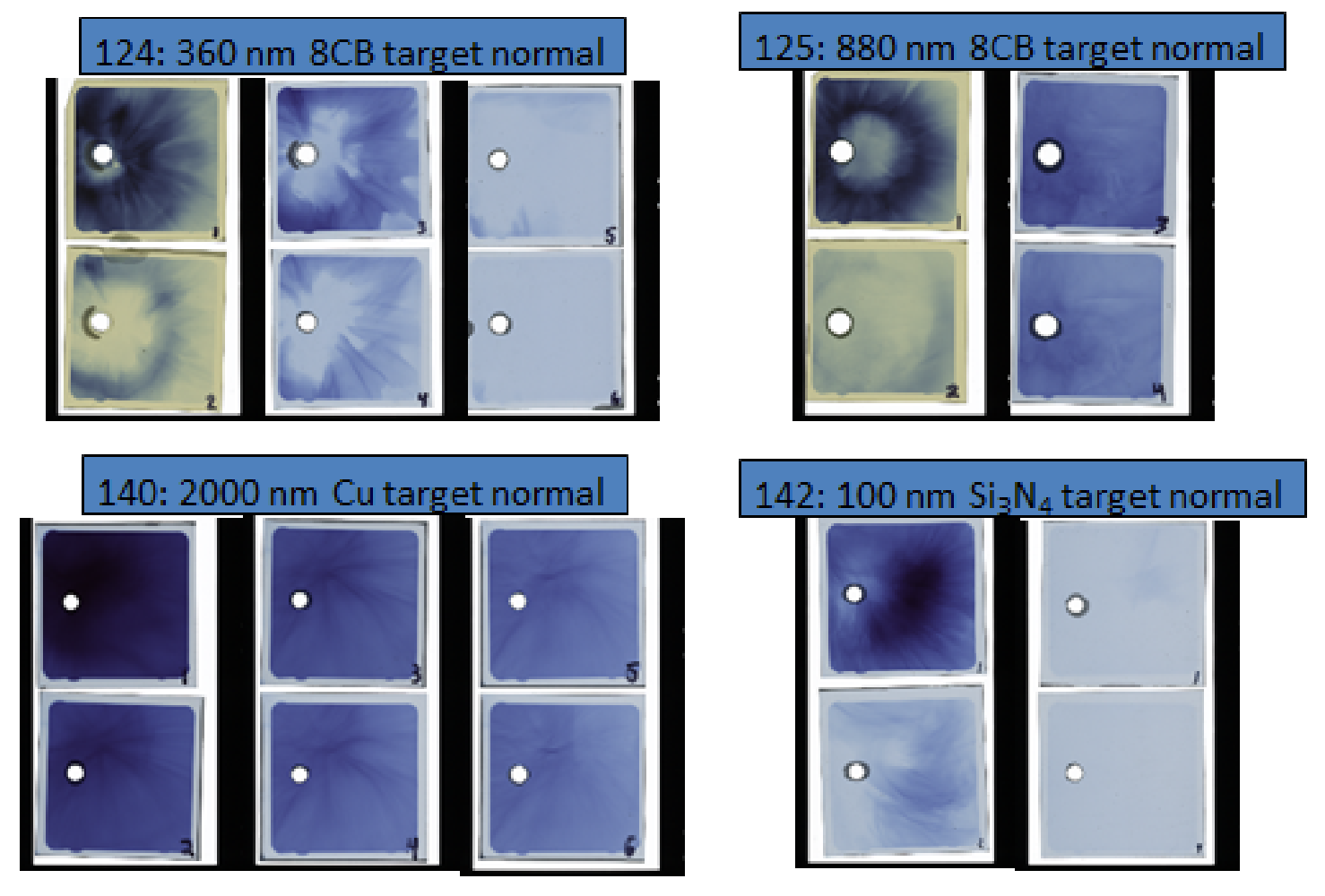

Figure 9.8: RCF stacks demonstrating the low-energy annular dose ring resulting from liquid crystal shots. This was not observed on the $\mathrm{Cu}$ and $\mathrm{Si}_{3} \mathrm{~N}_{4}$ targets taken during this run. 
Even in the picture described above one would expect the annular region to be filled in on the deeper layers of the RCF stack. This is not the case, as can be seen in Fig. 9.8: the annular region persists for the first two to four sheets, by which point the signal is either gone or consistently low across the entirety of the fifth and sixth layers. Additionally, the defocused nature of these shots presents a more flat-top and less Gaussian profile of incoming laser on target, which would tend to produce more uniform proton spatial distributions in general.

Another possible explanation for this annular spatial distribution of the low energy protons comes from the fast electrons generated as part of the TNSA process. Once these electrons leave the rear side of the target, some of them will undergo a process known as refluxing where they are pulled back toward the solid density target by the same sheath field that is accelerating rear-side ions. These electrons will continue to swing back and forth through the target, accelerating bunches of ions as they travel. There is some divergence to be expected in this case, resulting in a widening region of refluxing electrons as they make more passes through the target. For appropriately thick targets this refluxing ion acceleration may cause rings of proton signal to be visible, with outer rings containing fewer protons and with lower energies because they were accelerated by a smaller number of more spread out electrons.

This effect has been seen before, primarily from longer pulses (hundreds of $f s$ ) and thicker targets $(>10 \mu \mathrm{m})$, where it has been attributed to electron refluxing.[131] Here multiple rings of increasing radii can be seen and are attributed to the widening distribution of refluxing electrons upon multiple passes through the target. With the shorter pulse duration and target thickness on these experiments it is expected that a few rings will still be visible if this is the dominant effect causing them. In fact, only one ring was observed on any $8 \mathrm{CB}$ shot regardless of thickness, suggesting that this effect may have played some role in the formation of the annulus but was not the sole cause.

A final possibility for the annulus is the collisionless shock ion acceleration mechanism. Briefly, an electrostatic shock is induced at the front of the target by the electrons initially accelerated in the laser field. These electrons travel through the bulk of the target, and the shock they produce accelerates ions that cross it to up to twice the shock velocity. The mean free path for these electrons is much greater than the density discontinuity defining the shock, making the interaction collisionless.

This mechanism is currently under study as a method to achieve monoenergetic ion spectra because of its tendency to bunch ions into one or several groups of energies. Energy bunching of this sort can be seen in the raw spectrum on the left of Fig. 9.9. Here the target normal accelerated ions are shown as they appeared on the Thomson parabola spectrometer for two different $8 \mathrm{CB}$ shots. A sharp turn-on and exponential decay is indicative of TNSA, but the spectra lineouts shown to the right in Fig. 9.9 are both broader than expected 


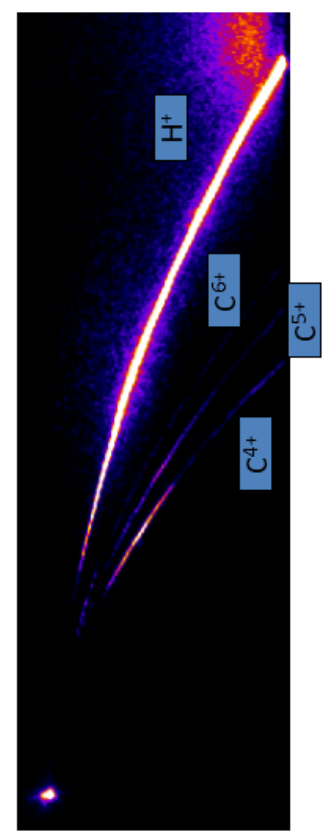

Shot 123: $1.3 \mu \mathrm{m} 8 \mathrm{CB}-$ Carbon ions

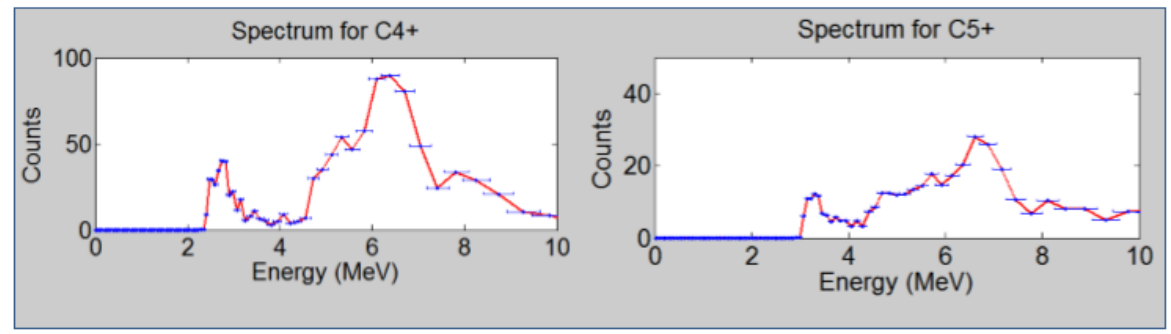

Shot 133: $890 \mathrm{~nm} \mathrm{8CB}-$ Carbon ions

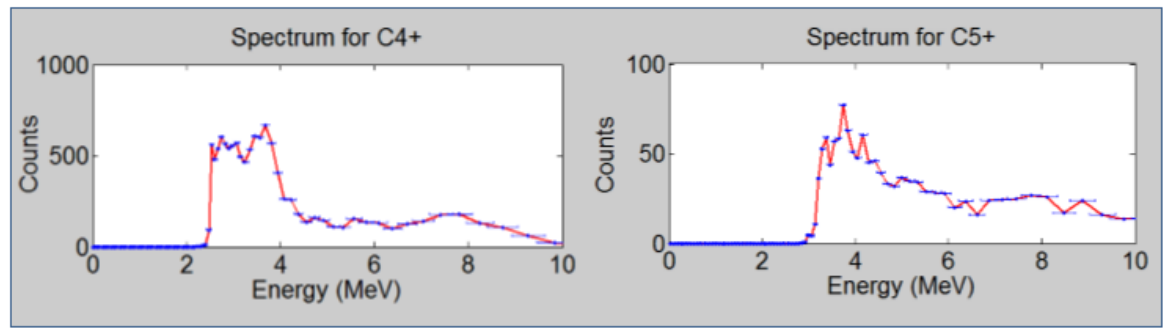

Figure 9.9: Thomson parabola trace for a shot that exhibited some energy bunching. The graphs are lineouts of some of the individual ion traces for different ionization states of carbon. Here sharp jumps can be observed in the cutoff for one species compared to the turn-on of the next. This can also be seen in the raw image scan at left, where the brightness changes sharply horizontally across ion species. Typical TNSA spectra do not exhibit these bunching features, suggesting another mechanism is contributing here.

and have subsequent jumps in energy, suggesting another process. This effect is greatly diminished or often not present at all on ion traces from other target materials.

Interestingly, simulations have shown that collisionless shocks are more prevalent for defocused beams, [132] as was the case for these shots. This larger spot size ensures that the transversely expanding plasma does not leave the shock width region, observed to be around $10 \lambda$ in simulations.

This does not entirely explain why the bunching and ring structures are more prevalent on $8 \mathrm{CB}$ targets than on the $\mathrm{Cu}$ or $\mathrm{Si}_{3} \mathrm{~N}_{4}$. A main difference is of course the composition of the target material. In previous simulations, different amounts and densities of light and heavy ions have been shown to have some modulating effect on the accelerated ion spectrum. $[62,133]$ It's possible that the similar densities of hydrogen and carbon in the $8 \mathrm{CB}$ films contribute to the energy bunching, although more investigation and simulation is necessary to make a firm judgment. 


\subsection{Conclusion}

Plans to repeat this experiment with a thinner handoff target and thus a much smaller spot size and higher intensity on target are underway at the time of this writing. The new handoff target consists of a $100 \mathrm{~nm} \mathrm{Si}_{3} \mathrm{~N}_{4}$ membrane coated with nanoparticles in a similar procedure to the glass slide handoff targets, but here the thinner material results in negligible refractive shift on the length scale of the $10 \mu \mathrm{m}$ short pulse Rayleigh range. Additionally, new RCF stacks have been designed to reveal protons with higher energies than the $13 \mathrm{MeV}$ cutoff for this experiment, since it is clear that higher energies of protons were produced even with the defocused shots. Furthermore, variably defocused shots would shed light on which of the mechanisms discussed above were responsible for the results of the run presented here.

On top of these experimental successes, the ease of liquid crystal film formation suggested strongly that they could be used as high repetition rate targets. The next Chapter describes the design of a device to form these films rapidly in vacuum. 


\section{Chapter 10 \\ High Repetition Rate Liquid Crystal Film Target Inserter}

\subsection{Overview}

The experiments detailed so far have demonstrated liquid crystal films as variable thickness planar targets advantageous for laser matter experiments including ion acceleration. By controlling the parameters of film formation their thickness can be varied on-demand from $10 \mathrm{~nm}$ to above $10 \mu \mathrm{m}$. Previous experiments with these targets used them in single shot configuration, requiring chamber cycling after the pre-made films were expended. Presented here is a film formation device capable of drawing films from bulk liquid crystal volume to any thickness in the aforementioned range. This device will form films under vacuum to within $2 \mu \mathrm{m}$ of the starting position, well within the Rayleigh range of even tight $F / \#$ systems. The repetition rate of the device approaches $0.1 \mathrm{~Hz}$ and varies thickness by controlling temperature and volume used during film formation. The details of the device and its characterization are presented here.

\subsection{Introduction}

As ultra-intense laser technology improves, new facilities are able to achieve progressively higher repetition rates. The BELLA facility at Lawrence Berkeley National Laboratory (LBNL) is a currently operating petawatt facility with a $1 \mathrm{~Hz}$ repetition rate,[134] and the Extreme Light Infrastructure (ELI) facilities under construction will have petawatt lasers at the $10 \mathrm{~Hz}$ rate. Increased shot rates promise higher flux for experiments and applications, as has been discussed in previous Chapters.

Of the possible solutions presented in Chapter 3, none of them provide quick or easily controlled thickness variation. This is a powerful tool both to optimize the desired interaction to laser parameters such as energy, pulse width, and contrast, as well as to access acceleration mechanisms that rely on target thickness such as Target Normal Sheath-field 
Acceleration (TNSA), Radiation Pressure Acceleration (RPA), and others.

Liquid crystals can be formed into membranes that preserve planar geometry while adding the considerable benefit of on-demand thickness variation between a few nanometers and several microns.[135] Their low vapor pressure allows target formation and thickness manipulation at typical target chamber pressure levels, and the low volume per film and cost per volume renders them ideal targets for long-term high rep-rate use. To this end an apparatus was designed that forms liquid crystal films under vacuum with the temperature and volume control necessary to achieve the $n m$ to $\mu m$ thickness range demonstrated in recent single-shot experiments. The device consists of a vertically sliding rail that draws a liquid crystal film as it moves across a $4 \mathrm{~mm}$ aperture within a copper frame; as such the device is called the Linear Slide Target Inserter (LSTI).

\subsection{Linear Slide Target Inserter design}

Films have previously been formed by hand simply by dispensing a small volume of liquid crystal next to an aperture and then dragging it across with a stiff wiper. To both control the thickness and obtain a uniform film, temperature resolution over a few degrees with regulation to better than $1.0{ }^{\circ} \mathrm{C}$ and volume management down to $100 \mathrm{~nL}$ are required, so these controls need to be built into a high repetition rate device as well.

Single-shot films were made on a setup bench and then transferred to the vacuum chamber which was subsequently pumped down to the $10^{-6}$ Torr level before final target alignment. Throughout this process the film thickness did not change. However, high repetition rate shots necessitate in-vacuum film formation, since only a finite number of copper flags or target frames can be loaded within the chamber at a time.

To this end a film formation device was designed that could be installed on a target positioner apparatus at Target Chamber Center (TCC). The device is shown in Fig. 10.1. The primary pieces are a copper frame with a single $4 \mathrm{~mm}$ diameter aperture bored near its top. A copper wiper sits flush with this frame and can be moved across the aperture by a linear drive motor. By placing a small known volume of liquid crystal underneath the wiper a film can be drawn: the target material is pushed up to the frame aperture and then pulled back down by the wiper motion.

Since accurate temperature control is paramount to thickness manipulation both the frame and wiper of the device are primarily made of copper. The frame has a $4 \mathrm{~mm}$ diameter clearance hole to act as an aperture for film formation. The back side of this aperture has a $45^{\circ}$ bevel to allow attenuated, unamplified short pulse light to be collected and characterized on the in-situ focal spot alignment camera just behind TCC. This aperture bevel has the added benefit of moving a forming film to its edge, providing repeatable film positioning as will be described below. 

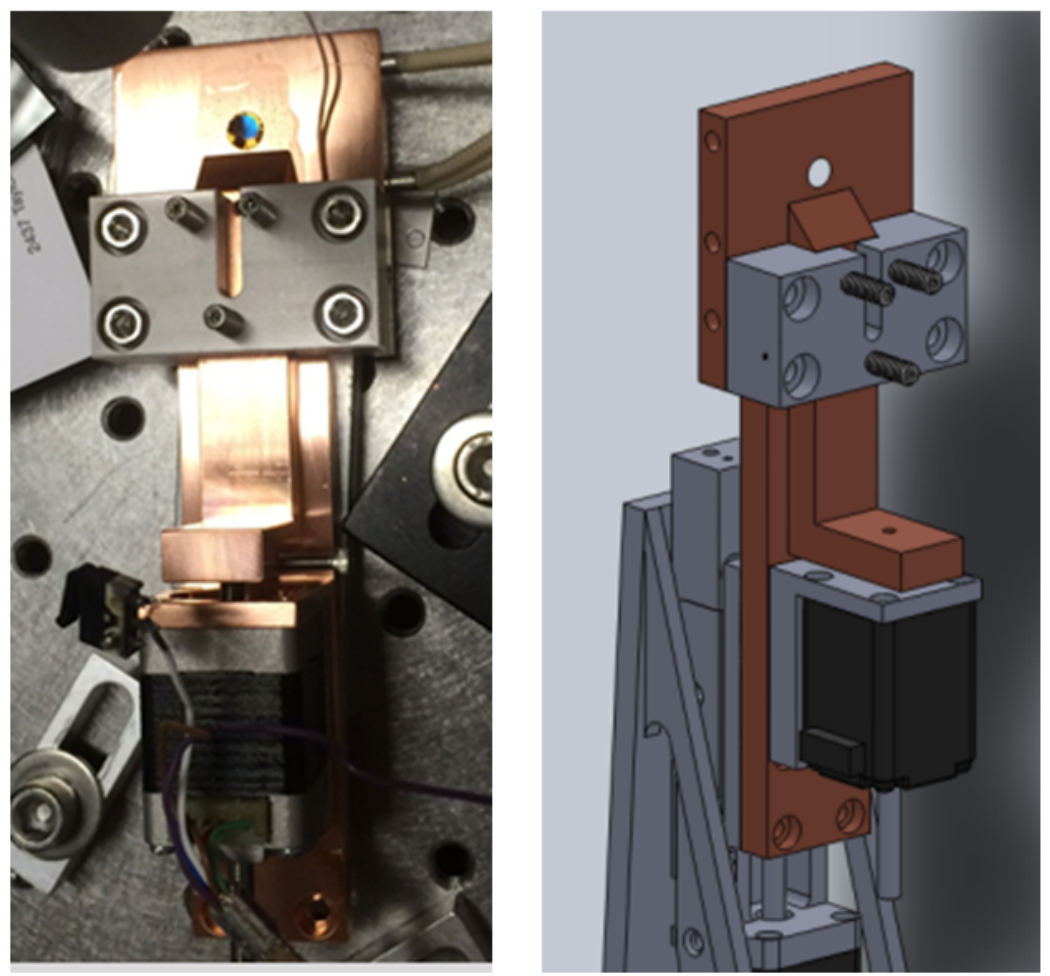

Figure 10.1: Schematic of LSTI. A vacuum-compatible motor moves a wiper vertically with variable speed to form a film within the $4 \mathrm{~mm}$ diameter aperture. Horizontal channels allow for temperature change via a chiller water line and monitoring via type $\mathrm{K}$ thermocouple. The clearance hole in the wiper allows for additional liquid crystal volume deposition. 
The wiper is another copper piece with a beveled top edge that can be pushed vertically with a full travel of $50 \mathrm{~mm}$ by a NEMA 8 vacuum motor (Hayden Kirk). The wiper is held flush to the frame by a polyether ether ketone (PEEK) bridge. This piece has three Delrin-tipped spring-loaded plungers to provide a variable amount of force pressing the wiper down onto the frame, as a firm connection is necessary for film formation.

The volume of liquid crystal present in a single film of sub- $\mu m$ thickness is on the order of $10 \mathrm{~nL}$, so the application of the precise volume for just one film is challenging. A $2 \mathrm{~mm}$ diameter clearance hole is placed in the wiper for the application of liquid crystal directly to the space between the wiper and frame. This can be done with tubing connected from this clearance hole to a precision syringe pump (Harvard Apparatus) for fine control of volume deposition. In practice, it is often easier to simply apply a volume on the order of $1 \mu L$, significantly more than that required for one film, and to vary other film formation parameters to control thickness. In this way one application of liquid crystal volume before chamber evacuation can provide hundreds of films before more volume is needed, in this case bypassing the need for the syringe pump and small inner diameter tubing on the vacuum chamber.

This large initial volume deposition has the additional benefit of preventing scratching between the two copper surfaces of wiper and frame. With insufficient lubrication, over several wiper draws grooves will grow into channels that change the liquid crystal volume present near the film aperture, hindering full thickness control. To minimize these surface effects both the wiper and frame pieces are polished with successively fine grains of sandpaper from 160 to 2000 grit, followed by polishing compound to a mirror finish. Maintaining this level of surface smoothness increases the thickness repeatability significantly.

An alternative measure taken to reduce frame scratching was to modify the wiper to include a Teflon piece affixed to the bottom of the wiper. With this design scratches only form on the Teflon wiper, and only over a large number of film draws. While the Teflon cover ensures greater film thickness control through scratch prevention for many more draws than the pure copper wiper, the reduced adhesion of liquid crystal to the Teflon surface results in slightly different film formation behavior, as will be discussed below.

As discussed in previous chapters, thickness control requires precise temperature modulation and monitoring. In the vacuum environment heat will eventually accumulate from the motor that drives the film wiper and spread up to the film area, resulting in a temperature gradient that produces uneven films and in general impedes thickness control. To accommodate this, two $3 \mathrm{~mm}$ diameter clearance holes are bored at different heights horizontally through the wiper. These can be used for resistive cartridge heaters as on previous frame designs, but here the heating needs to be reduced since that from the motor is too great to dissipate easily in vacuum. In this case the clearance holes are to be used 

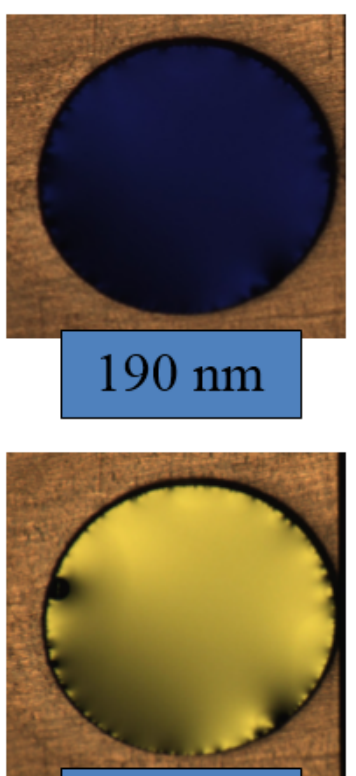

$300 \mathrm{~nm}$

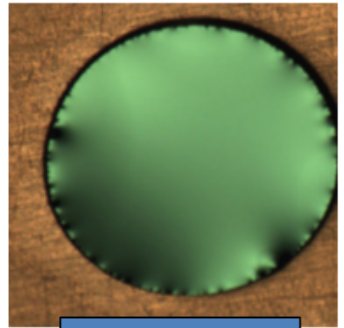

$260 \mathrm{~nm}$

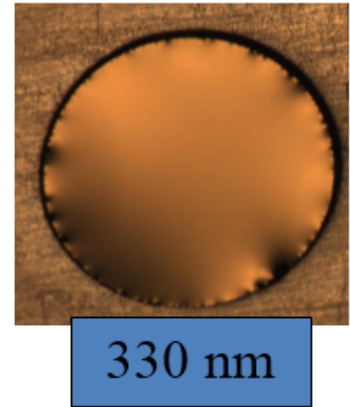

Figure 10.2: $4 \mathrm{~mm}$ diameter films made with the LSTI to demonstrate fine thickness control. Shown are four films separated in thickness enough to show different colors, though finer thickness gradations are possible with this film formation device.

for the insertion of a copper tubing line connected through the chamber wall to a small water chilling unit. A third channel allows a type $\mathrm{K}$ thermocouple to be inserted internal to the frame near the film formation aperture. In this way the frame temperature can be maintained to within $0.1^{\circ} \mathrm{C}$ of the desired temperature regardless of the variable thermal load from the motor.

With these considerations the same thickness control available for single-shot films can be achieved with the LSTI. Figure 10.2 shows four films made with this device. The high quality aperture and polish of the wiper and frame reduce the meniscus region to its minimum and ensure a uniform thickness film formed in any orientation.

\subsection{Film control}

\subsubsection{Position repeatability}

Film thickness as described here is again measured with the Filmetrics spectral reflectance device. This measurement has a $50 \mathrm{~ms}$ integration time so it can be used at high repetition rates and has an accuracy of $2 \mathrm{~nm}$ with proper calibration.

Of note is the repeatability of the film formation plane of the LSTI. A digital linear micrometer was used in conjunction with the Confocal High Intensity Positioner (CHIP) 
target alignment device to determine the film location upon film destruction and subsequent reformation. A film was placed at the best focus of the CHIP, then a new film was drawn and the target frame was moved until it was measured to again be at best focus of the alignment device. The digital micrometer recorded the net displacement in the target plane during this $z$ realignment. The RMS value of film position was under $2 \mu m$, well within the $10 \mu \mathrm{m}$ Rayleigh range of the $F / 2$ Scarlet laser.

The reason for this repeated film formation position lies in the beveled aperture placed in the copper frame. As the wiper is drawn down, a film initially forms with its upper edge connected to the copper frame and its lower edge still connected to the wiper. Here the wiper sits some small amount (sub- $m m$ ) above the plane of the copper frame due to the effect of polishing the wiper edge. Because of this the film begins at an angle with respect to the copper frame (and its eventual resting plane). The surface tension of the liquid crystal is such that the wiper pulls the film up towards the wiper as it moves past, effectively shifting the liquid crystal film to be on the extreme front edge of its frame as it is being formed. As the wiper moves down, this angle between the film and the frame plane decreases. As the wiper edge leaves the aperture area the film will snap down into place, now fully contained within the frame aperture. In this way films are brought to the same plane within $2 \mu \mathrm{m}$ of the previous film location each time.

To further test this, films several $\mu m$ thick were formed using the slowest LSTI motor speeds. The location of the front surface of such film was a distance from the previous film position by a value equal exactly to its thickness, as measured by the Filmetrics device. This was done consistently for many films, indicating that the back surface of each film forms in a stationary spot within the frame aperture.

Evidence of this film-pulling by the wiper is shown in Fig. 10.3. Since the vertically displaced wiper is acting as one edge of the film being drawn, the film is changing its angle as the wiper moves further right in the diagram. This is seen explicitly in Fig. 10.3c: here the wiper appears as a dark bar moving left to right. This camera is positioned to see colored reflection from a film only if it is within the plane of the frame. As such the film appears black to the camera initially, indicating its angle is not yet correct for reflection to the camera. As the wiper moves right the forming film changes angle to be closer to co-planar with the aperture, and more of the film becomes visible on the camera. The film is then fully visible once it ceases to have the wiper as one edge.

\subsubsection{Thickness control}

Although temperature, applied volume, and surface polish all have some effect, for practical film repeatability wiper speed is the quickest and most convenient method for tuning film thickness. For example, while the volume applied demonstrably governs the upper limit to thickness produced at any speed, sub-100 $\mathrm{nm}$ films can still be produced when the applied 
a)

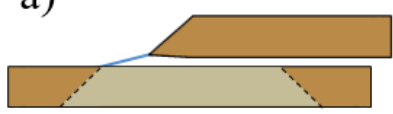

c)

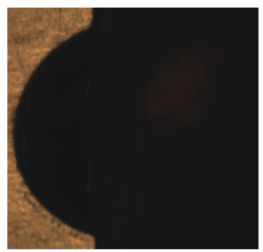

b)
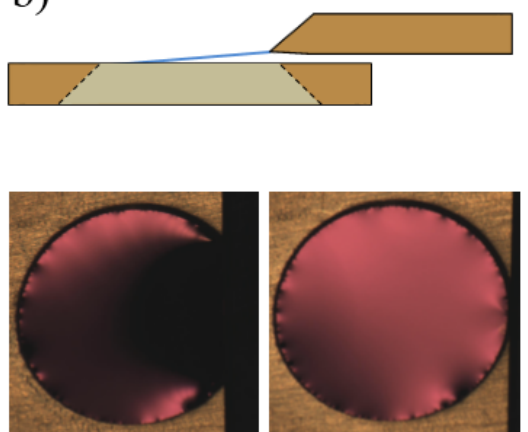

Figure 10.3: The geometry of the beveled aperture and raised wiper provide a consistent film formation plane. a) A film initially forms between the aperture and the wiper, such that it is being pulled at an angle with respect to the aperture plane. b) As the wiper continues drawing, more of the film is transferred to front edge of the beveled aperture. c) Snapshots taken during film formation. As the wiper moves left to right the forming film is brought closer to co-planar with the frame aperture, resulting in the correct angle for observing the pink color stemming from constructive interferences at this film thickness of $530 \mathrm{~nm}$.

volume is greater than $10 \mu \mathrm{L}$. Furthermore, while in general a higher temperature results in thicker films, even at temperatures of $29.5^{\circ} \mathrm{C}$ (near the smectic/nematic phase transition) a sufficiently fast wiper speed will still generate a thin film.

The effect of wiper speed can be seen in Fig. 10.4, where three regions of film formation have been highlighted. Region III, at the fastest wiper speeds, produces a sub-100 $\mathrm{nm}$ film with precision within $10 \mathrm{~nm}$ each time. The thickness formed in this region is only a weak function of the applied volume and temperature. Film formation at these wiper speeds provide high repetition rate films and is ideal for applications where a consistent, thin target is desired. The relatively large surface tension inherent to the smectic liquid crystal phase allows film formation even at quite high wiper speeds. As a result the maximum draw time is limited currently by the choice of motor, not the liquid crystal itself. With top motor speeds a thin film can be formed at a repetition rate of about $0.3 \mathrm{~Hz}$.

The base film thickness formed in Region III is chiefly a function of the manner in which a film is held to the wiper as it draws the film. For example, the Region III thickness is consistently $30 \mathrm{~nm}$ when using a copper wiper on a copper frame, but changes to $75 \mathrm{~nm}$ when the wiper contact surface is changed to Teflon. The details of this interaction are still being studied, including how the shape and quality of the wiper edge effects this Region III thickness.

Regions I and II both have less precision at a given wiper speed. The vertical bars in Fig. 10.4 indicate the range of film thicknesses produced at the given wiper speed, while the dots show the average over these thicknesses. Region II occurs in the few hundred 


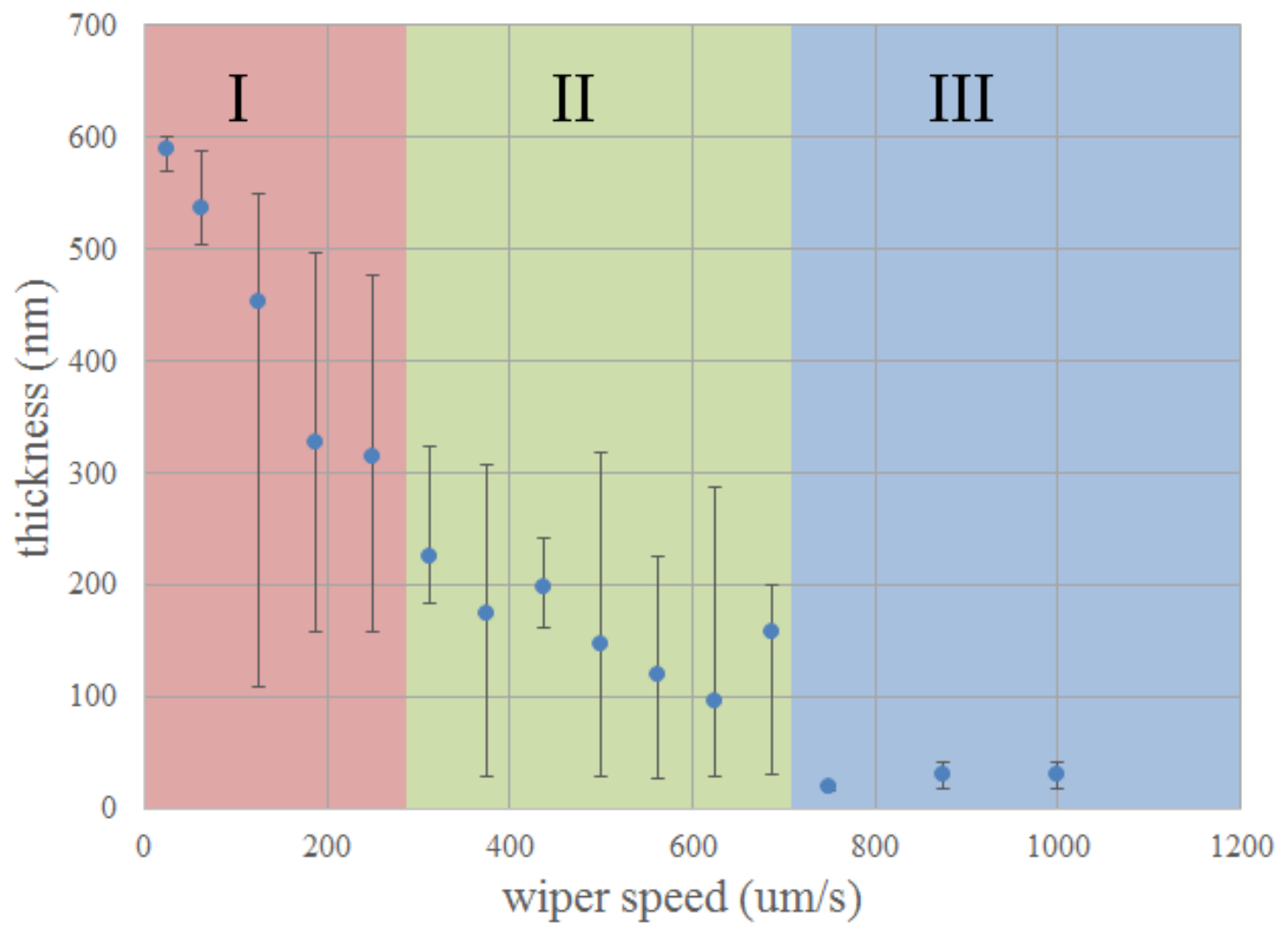

Figure 10.4: Control of film thickness via LSTI wiper speed. Here the vertical bars show the range of films formed over five draws at each speed, while the dots indicate the average of those films. This average increases in thickness as the wiper speed decreases. The shaded areas indicate three different regions of thickness range, discussed in the text. 
$\mu \mathrm{m} / \mathrm{s}$ wiper speed range, varying slightly with applied volume and temperature. Here film thicknesses are typically between 100 and $500 \mathrm{~nm}$, changing as a stronger function of volume than in Region III. Region II clearly has a larger potential film thickness range at a given wiper speed than Region III, but in general this average thickness now increases as the wiper speed decreases.

Region I is typically below $100 \mu \mathrm{m} / \mathrm{s}$ wiper speed and results in film thicknesses up to several $\mu \mathrm{m}$, where this maximum possible thickness is correlated more directly to the volume of liquid crystal applied. The thickness range bars in Fig. 10.4 are smaller here not necessarily because this Region exhibits greater precision, but rather because the slow film formation speeds (around 60 seconds) amount to significant heating from the wiper motor in vacuum, which then requires some time to be corrected by the chilling unit. This is, however, the best method for generating films on the order of tens of $\mu m$, which would most likely be of use on lower repetition rate experiments regardless.

The increased possible thickness range of Regions I and II necessitated an investigation into the practical technique for producing a desired thickness. Volume control is a strong tool for this when forming single-shot films manually, but a large volume of liquid crystal is applied between the LSTI wiper and frame to prevent scratching and for prolonged vacuum use. An approximate method of volume control is to change how far above the film aperture the wiper moves (left in Fig. 10.3c): this will determine how much of the liquid crystal volume clinging to the wiper will be brought to the film aperture before drawing. For example, beginning a draw from $1.5 \mathrm{~mm}$ above the aperture will result in a thicker film than if the initial draw is from $0.5 \mathrm{~mm}$ above the aperture, simply because of the extra volume brought into the frame aperture region. In this way the thicker range at a given wiper speed in Fig. 10.4 can be preferentially accessed by beginning a draw from higher up on the frame.

If a film formed is instead thicker than desired, a similar technique can generate a subsequently thinner one. Here the process is to move the wiper again over the aperture area at a slightly faster speed, effectively wiping away some of the smectic layers of the film.

Though films formed in Regions I and II do not have the precision of those from Region III, the thickening and thinning techniques outlined above allow the desired film thickness to be approached over only a handful of wipes. By choosing the appropriate wiper speed, the desired thickness can be reached within ten percent in only a few draws, with increased precision possible as the film is re-drawn. Since each draw takes a matter of seconds, multiple re-draws are easily feasible for most current high power laser systems. For example, the LSTI and its associated thin film formation techniques enable Scarlet to achieve shots at its full repetition rate of one per minute for the first time. Additionally, the stability of the smectic liquid crystal phase combined with its low vapor pressure enables films formed within the LSTI to maintain their thickness nearly indefinitely, making them ideal as well 
for laser systems with repetition rates of once every few minutes to hours.

\subsection{Conclusion}

The in-situ thin film target positioner presented here offers liquid crystal film formation in vacuum with on-demand thickness variation from $10 \mathrm{~nm}$ to over $10 \mu \mathrm{m}$ at a repetition rate of $0.3 \mathrm{~Hz}$. The device uses the liquid crystal 4-cyano-4'-octylbiphenyl (8CB), and so is ideal for high intensity laser experiments that rely on low- $Z$ targets such as ion acceleration. The low vapor pressure, low cost per volume, and thickness variability make targets formed with this device ideal for many high intensity, moderate repetition rate laser applications. Films formed with this device are of uniform thickness due to careful control of temperature, liquid crystal volume, wiper and frame polish, and wiper speed as detailed here. 


\section{Chapter 11 FutureWork}

\subsection{Impact of Work}

This work constitutes the development and first experiments on liquid crystal targets for intense laser experiments. As has been discussed, the ability to both select and tune the laser interaction details like the mechanisms of ion acceleration constitutes an important advancement for the field. Initial experiments have been performed to investigate the differences between various ion acceleration mechanisms that depend on target thickness. Additionally, the formation of these films at high repetition rate has been demonstrated. Coupled with the low cost of liquid crystal, these properties make viable a number of applications and experiments that were not before their development.

Interest in the films has already flourished, especially among European laser scientists where a number of high power high repetition rate laser systems are currently under development. What follows in this section is a brief overview of some of the experimental ideas that will be pursued with liquid crystal films over the next year. Many of these experimental ideas already have dedicated laser time at facilities around the world.

\subsection{Upcoming experiments}

\subsubsection{Film metallization}

In their current form liquid crystal films are excellent low- $Z$ ion acceleration targets. For the many applications that rely on proton and similar light ion acceleration (e.g. hadron cancer therapy, neutron generation) this sort of target is ideal. However, there are many other applications that require a high- $Z$ target (e.g. x-ray generation, optimized electron acceleration). As such the prospect of metallizing liquid crystal films has been explored to some degree already.

One possibility lies in changing the nature of the liquid crystal itself. The currently used $8 \mathrm{CB}$ is a long-chain hydrocarbon molecule that manifests the nematic and smectic 
A phases, but there are hundreds of other types of liquid crystals. Of particular note are the metallomesogens, [110] which contain a metal ligand as their central structure. A metallomesogen that has a smectic phase could be used to make freely suspended films with the techniques outlined in this work. The downside would be that other lighter elements would most certainly still be present in the target, potentially complicating the physics at play (see for example the discussion at the end of Chapter 9. Additionally, films made with metallomesogens will have a lower metal density than typical foil targets, potentially complicating some applications.

Another option is to make a mixture of liquid crystal and metallic substance, either before or after drawing the film. Candidates for this procedure include metallic nanoparticles, which are frequently used in liquid crystal films formed on a substrate in order to enhance their response to external fields. Even dispersal of such nanoparticles may require another substance to coat the nanoparticles and prevent clustering. The issue here is ensuring that the added nanoparticles do not interfere with thin film formation or with thickness control. The layered nature of smectic films tends to squeeze defects out from within the structure, and the same seems to happen for nanoparticles of certain sizes.

A third option is directly applying metal to a film, using the liquid crystal as a substrate. This would be nearly impossible with a soap and water film, for example, but not so with liquid crystal films due to their relatively high stability. Initial tests of copper ion sputtering have been performed on freely suspended $8 \mathrm{CB}$ films with some success. Though the resulting copper layer was uneven, perhaps due to the large amount of material $(500 \mu \mathrm{m})$ applied, the liquid crystal film did survive long enough to act as a substrate for the copper layer, as can be seen in Fig. 11.1. More work needs to be done to perfect such methods, but the possibility of variable thickness metallic films coated onto freely suspended liquid crystal substrates is exciting and promising.

\subsubsection{Laser pulse modification}

As mentioned previously, laser pulse contrast is an important quantity to diagnose for any laser experiment. Prepulses and a poor contrast pedestal can cause an unknown and often unwanted preplasma to form on the front of a target, modifying the ensuing laserplasma interaction. One way to improve the contrast of an existing laser system is to put a transmissive, optically flat material in the path of the partially focused beam. Any prepulses will transmit through the optic, but the focus is tuned such that sufficient intensity is reached within the main laser pulse to create a plasma on the front of this material. The critical surface from that plasma will reflect the remainder of the pulse, ridding it of the prepulse that transmitted through the material before a plasma was there for reflection. Such a device is called a plasma mirror, and is currently used on many high intensity systems. This typically increases the contrast of the pulse by two orders of magnitude in 


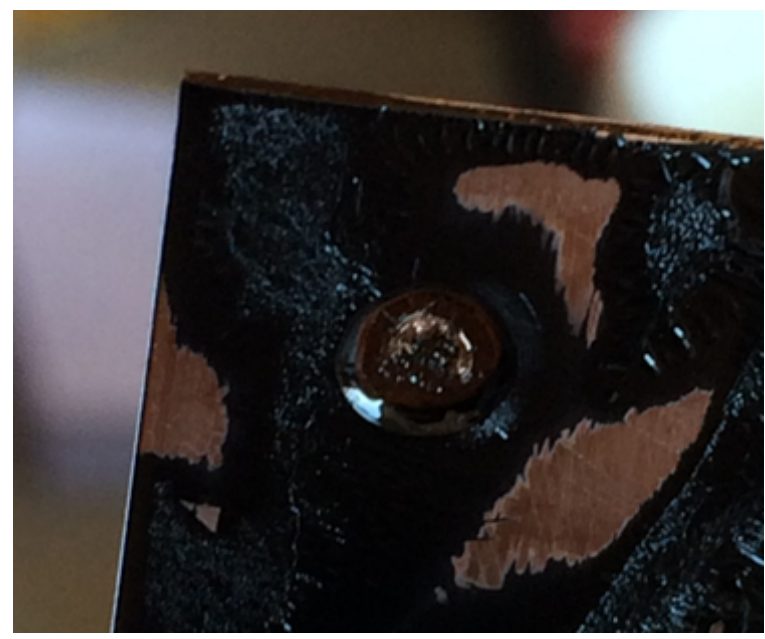

Figure 11.1: $500 \mu \mathrm{m}$ of copper ions sputter coated onto a $4 \mathrm{~mm}$ diameter liquid crystal film. The result is a black, uneven surface within the frame aperture, but evidence that the liquid crystal film survived long enough to act as a substrate for the metal ions.

intensity. The downside to plasma mirrors is twofold: first, as much as $30 \%$ of the incoming energy is lost in the plasma formation and subsequent reflection, and second that reflected light often has a poor mode quality due to the unevenness of the reflecting plasma surface. Plasma mirrors are used despite these drawbacks-often in pairs to increase the contrast further-because of how important a clean laser pulse is to many modern experiments. [37]

The formation of a plasma surface on such a mirror renders that location unusable on subsequent shots, so the device must be translated and eventually a new piece of glass must be exchanged for the used one. As such plasma mirrors are time-consuming to set up and use and can be expensive, especially if they are optimized with an antireflection coating.

Liquid crystal films offer an inexpensive, reflective surface for plasma mirror use. Additionally, the thickness of the film could be tuned to the appropriate antireflection coating value of the incoming laser wavelength, further increasing the contrast improvement. A cartoon of the idea is shown in Fig. 11.2.

A similar idea exists for improving the pulse contrast in transmission rather than reflection. Here a disposable liquid crystal film would be place a small distance away from the main target. Any prepulse in the beam would hit the disposable film first and be absorbed or reflected. The subsequent main pulse would then transmit through the remnants of the disposable target, either conventionally because of the reduced density of the destroyed initial target, or via the increased intensity of the main pulse inducing relativistic transparency, to be described in the next section. This is not a new idea for High Energy Density Physics (HEDP), but was unfeasible previously because the thickness of the disposable film must be tuned to adequately remove prepulse. 

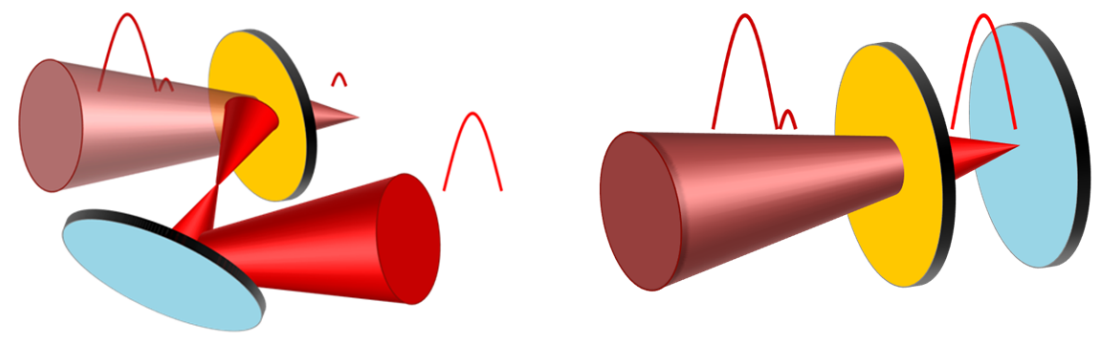

Figure 11.2: Left: Plasma mirror concept using liquid crystals. Here the yellow film is transparent to the prepulse but becomes reflective once the main pulse creates a critical density plasma on it. Right: Plasma filter concept, wherein the prepulse is absorbed or reflected by the yellow film, and the main pulse pushes through this reduced density to the main target.

\subsubsection{Relativistically Induced Transparency}

Ion acceleration mechanisms effective for sub- $\mu m$ targets will rely on or be affected by relativistically induced transparency (RIT). This phenomenon occurs when the relativistic plasma generated by an intense laser influences the local plasma frequency such that the pulse can travel through what would otherwise be an overdense region of the plasma. Recalling the plasma frequency listed in Chapter 2:

$$
\omega_{p}=\sqrt{\frac{n_{e} e^{2}}{\gamma_{L} m_{e} \epsilon_{0}}} .
$$

A sufficiently intense laser will oscillate electrons in the plasma at the front of a target to relativistic speeds. At this point the $\gamma_{L}$ is greater than 1 which reduces the plasma frequency and can thereby allow laser penetration beyond the classical critical surface. For sufficiently thin targets this will allow the laser to travel entirely through the target, potentially dragging some electrons and ions along with it to be accelerated in the laser propagation direction. A number of simulations and a few initial experiments have been done to investigate this effect on different laser systems and its potential utility for accelerating monoenergetic ion beams.[38, 39]

Despite the growing library of simulation and theory backing up RIT, to date there has been no systematic study of its onset as a function of intensity or target thickness. Varying target thickness presents a difficulty for such an experiment due to the relatively high cost and fragility of targets thin enough to demonstrate this effect, as well as the problem of aligning targets of different thicknesses within a target chamber. When it is done these thickness scan experiments typically have very low shot rates and coarse thickness variation data points, making quantification of the RIT effect difficult. 
The on-demand thickness control of liquid crystal films makes them ideal targets for a RIT onset experiment. The setup of such an experiment is outlined in Fig. 11.3, and will be implemented on Scarlet in the near future. The idea is to observe the transmitted light through a target as a function of the intensity of the laser pulse. Light moving through the target can be steered to a piece of Spectralon ${ }^{17}$, where the mode can be easily imaged with a camera situated outside the chamber. The graph here shows a possible transmitted signal as a function of laser intensity, where a large increase should be visible when RIT begins.
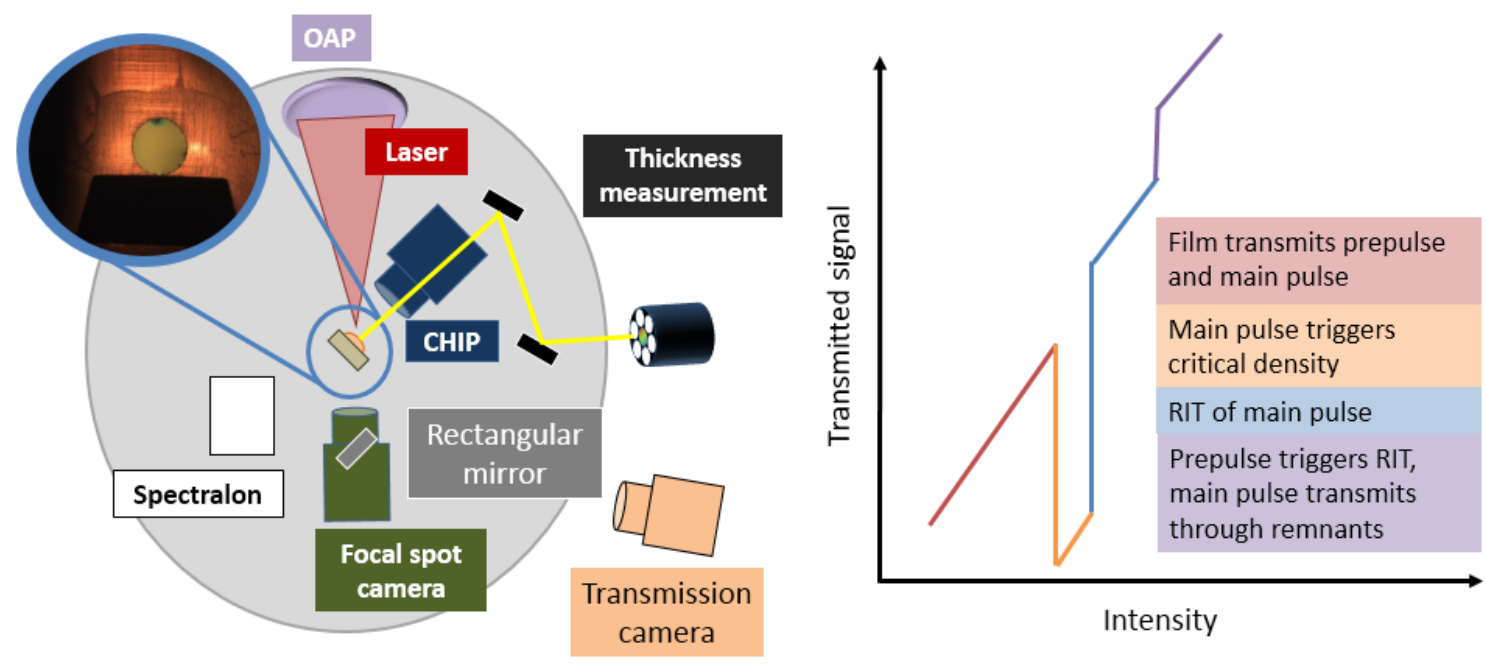

Figure 11.3: Left: Possible chamber setup for experiment investigating RIT, where the transmitted signal is observed on a Spectralon screen as a function of incident laser intensity. Right: Schematic graph of transmitted signal vs. laser intensity, with a large signal increase indicating the onset of RIT.

\subsubsection{Increased repetition rate for liquid crystal films}

\section{Spinning disk}

While the devices presented here are more than adequate for the repetition rates of the majority of existing laser systems, they still cannot quite match the $10 \mathrm{~Hz}$ rate that will be available to the newest facilities in the next 5 years. To this end, some effort has been expended to develop an even faster version of the LSTI. The same wiping mechanism has been implemented in a rotational geometry, where a disk with apertures spins beneath a

${ }^{17}$ Spectralon is a highly Lambertian scatterer material similar to Teflon. Its scattering properties make it useful for observing the mode of an intense laser beam without requiring the detector to be along the specular reflection direction, for example. 
stationary blade. A still of the device is shown in Fig. 11.4. The device current makes films at the $1 \mathrm{~Hz}$ level, and thickness control at these speeds is being developed.

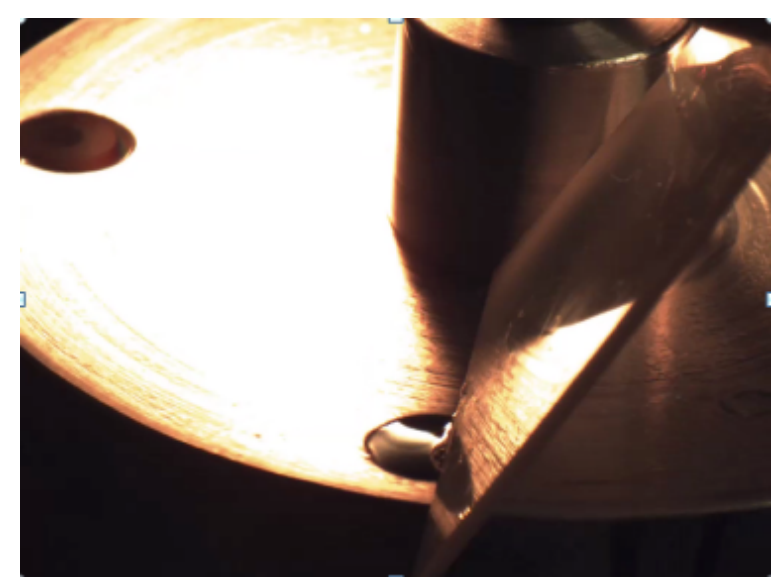

Figure 11.4: Spinning disk target inserter for $1 \mathrm{~Hz}$ and faster film formation currently under development. The device consists of a stationary Teflon blade with a spinning aluminum disk where films are formed underneath.

\section{Microfluidic jets}

The jet streams discussed in Chapter 3 have the capability of running at $k H z$ repetition rates, unlike current liquid crystal formation technology. It is possible that these designs could be adapted to work with liquid crystal, at which point the layering of the smectic film could aid in thickness control, and the low vapor pressure of $8 \mathrm{CB}$ would remove the differential pumping constraint of existing water jets.

This does not solve the issue of target planarity inherent to existing jets. An approach for generating a planar flowing jet that has been explored is to use the technology of a laser dye jet nozzle. Here a shaped aperture for the fluid flow would guide the jet just as it left the nozzle. Typically the nozzle cross section had a pinched feature in its center-when liquid was forced through this aperture at high pressure, a structure known colloquially as a "dogbone" developed just below the nozzle output. This feature has a roughly triangular flat region surrounded by thicker jet regions. Laser dye jet nozzles were never made to generate streams below the tens of $\mu \mathrm{m}$ level, and so are not directly useful for ion acceleration target formation.

However, modern microfluidic techniques are easily able to achieve $\mu \mathrm{m}$ and smaller dimension channels. Channels can be formed either by the standard ultraviolet photoresist mask creation and subsequent silicon bonding, or by etching the desired groove structure in 
some harder material. A water jet forced from such a microchannel can be seen in Fig. 11.5. This has already been demonstrated to generate $30 \mu \mathrm{m}$ thick planar jet regions with $\mathrm{mm}$ transverse dimensions from a similarly-sized channel.
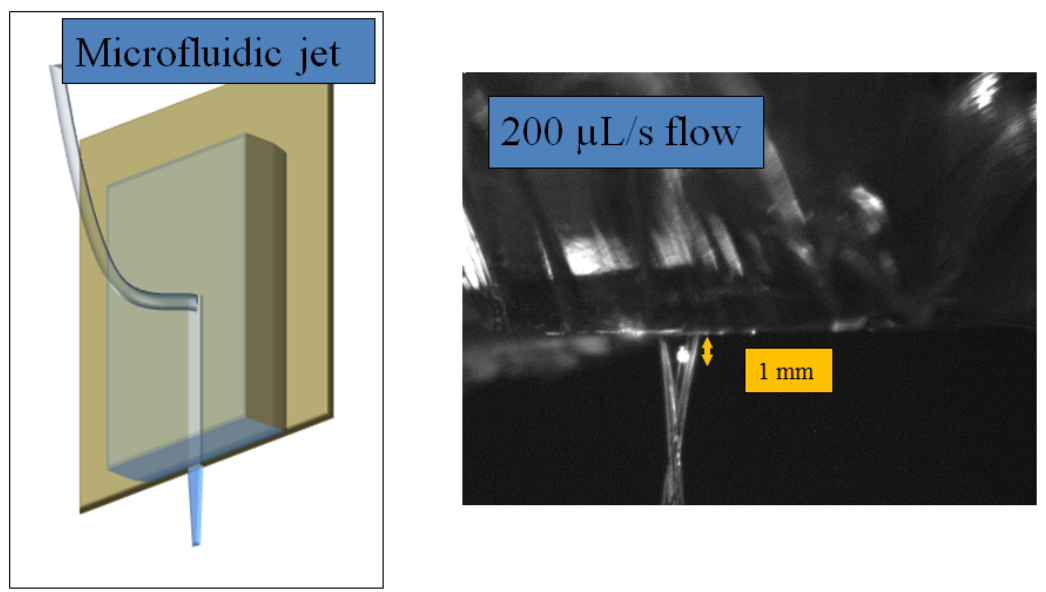

Figure 11.5: Microfluidic jet cartoon and microscope image. Here a deionized water jet is pushed through a microfluidic channel with a height of $30 \mu \mathrm{m}$ and width of $1 \mathrm{~mm}$. The resulting planar jet region is on the order of $1 \mathrm{~mm}$ as well, and has thickness roughly matching that of the channel height. Microfluidic channels made by Mike Prikockis of the R. Sooryakumar group at Ohio State.

\subsection{Conclusion}

Progress along the lines listed here and other continues on liquid crystal targets. Several offers of experimental time on laser facilities outside Ohio State have emerged due to the community's excitement for this advancement, such that much of the next year will be filled with experimental runs. I look forward to exploring fully the science these targets promote for HEDP and its related fields. 


\section{BIBLIOGRAPHY}

[1] M. Tabak, J. Hammer, M. E. Glinsky, W. L. Kruer, S. C. Wilks, J. Woodworth, M. E. Campbell, M. D. Perry, and R. J. Mason. Ignition and high gain with ultrapowerful lasers. Phys. Plasmas, 1(5), 1994.

[2] S. P. D. Mangles, C. D. Murphy, Z. Najmudin, A. G. R. Thomas, J. L. Collier, A. E. Dangor, E. J. Divall, P. S. Foster, J. G. Gallacher, C. J. Hooker, D. A. Jaroszynski, A. J. Langley, W. B. Mori, P. A. Norreys, F. S. Tsung, R. Viskup, B. R. Walton, and K. Krushelnick. Monoenergetic beams of relativistic electrons from intense laserplasma interactions. Nature, 431:535-538, 2004.

[3] M. Roth, D. Jung, K. Falk, N. Guler, O. Deppert, M. Devlin, A. Favalli, J. Fernandez, D. Gautier, M. Geissel, R. Haight, C. E. Hamilton, B. M. Hegelich, R. P. Johnson, F. Merrill, G. Schaumann, K. Schoenberg, M. Schollmeier, T. Shimada, T. Taddeucci,

J. L. Tybo, F. Wagner, S. A. Wender, C. H. Wilde, and G. A. Wurden. Bright laserdriven neutron source based on the relativistic transparency of solids. Phys. Rev. Lett., 110:044802, Jan 2013.

[4] M. I. K. Santala, M. Zepf, F. N. Beg, E. L. Clark, A. E. Dangor, K. Krushelnick, M. Tatarakis, I. Watts, K. W. D. Ledingham, T. McCanny, I. Spencer, A. C. Machacek, R. Allott, R. J. Clarke, and P. A. Norreys. Production of radioactive nuclides by energetic protons generated from intense laser-plasma interactions. Applied Physics Letters, 78(1), 2001.

[5] S. V. Bulanov, H. Daido, T. Zh. Esirkepov, V. S. Khoroshkov, J. Koga, K. Nishihara, F. Pegoraro, T. Tajima, and M. Yamagiwa. Feasibility of using laser ion accelerators in proton therapy. AIP Conference Proceedings, 740(1), 2004.

[6] M. M. Murnane, H. C. Kapteyn, and R. W. Falcone. High-density plasmas produced by ultrafast laser pulses. Phys. Rev. Lett., 62:155-158, Jan 1989.

[7] S. Kneip, C. McGuffey, S. R. Nagel, C. Palmer, C. Bellei, J. Schreiber, C. Huntington, F. Dollar, T. Matsuoka, V. Chvykov, G. Kalintchenko, V. Yanovsky, A. Maksimchuk, 
K. Ta Phuoc, S. P. D. Mangles, K. Krushelnick, and Z. Najmudin. Comparative study of betatron radiation from laser-wakefield and direct-laser accelerated bunches of relativistic electrons, 2009.

[8] H. Chen, S. C. Wilks, J. D. Bonlie, E. P. Liang, J. Myatt, D. F. Price, D. D. Meyerhofer, and P. Beiersdorfer. Relativistic positron creation using ultraintense short pulse lasers. Phys. Rev. Lett., 102:105001, Mar 2009.

[9] J. E. Bailey, T. Nagayama, G. P. Loisel, G. A. Rochau, C. Blancard, J. Colgan, Ph. Cosse, G. Faussurier, C. J. Fontes, F. Gilleron, I. Golovkin, S. B. Hansen, C. A. Iglesias, D. P. Kilcrease, J. J. MacFarlane, R. C. Mancini, S. N. Nahar, C. Orban, J.-C. Pain, A. K. Pradhan, M. Sherrill, and B. G. Wilson. High-density plasmas produced by ultrafast laser pulses. Nature, 517:56-59, 2015.

[10] P. Gibbon. Short Pulse Laser Interactions with Matter: An Introduction. Imperial College Press, 2005.

[11] G.E. Hogan, K.J. Adams, K.R. Alrick, J.F. Amann, J.G. Boissevain, M.L. Crow, S.B. Cushing, J.C. Eddelman, C.J. Espinoza, T.T. Fife, R.A. Gallegos, J. Gomez, T.J. Gorman, N.T. Gray, V.H. Holmes, S.A. Jaramillo, N.S.P. King, J.N. Knudson, R.K. London, R.P. Lopez, J.B. McClelland, F.E. Merrill, K.B. Morley, C.L. Morris, C.T. Mottershead, Jr Mueller, K.L., F.A. Neri, D.M. Numkena, P.D. Pazuchanics, C. Pillai, R.E. Prael, C.M. Riedel, J.S. Sarracino, A. Saunders, H.L. Stacy, B.E. Takala, H.A. Thiessen, H.E. Tucker, P.L. Walstrom, G.J. Yates, H.J. Ziock, J.D. Zumbro, E. Ables, M.B. Aufderheide, Jr. Barnes, P.D., R.M. Bionta, D.H. Fujino, E.P. Hartouni, H.-S. Park, R. Soltz, D.M. Wright, S. Balzer, P.A. Flores, R.T. Thompson, A. Pendzick, R. Prigl, J. Scaduto, E.T. Schwaner, and J.M. O'Donnell. Proton radiography. In Particle Accelerator Conference, 1999. Proceedings of the 1999, volume 1, pages 579583 vol.1, 1999.

[12] G. M. MacGillivray. Imaging with neutrons: the other penetrating radiation, 2000.

[13] U. Amaldi and G. Kraft. Particle accelerators take up the fight against cancer. Cern Courier, 2006.

[14] A. Sanchez K. F. Wall. Titanium sapphire lasers. Lincoln Laboratory Journal, 3(3):447-462, 1999.

[15] S. Le Pape, Y. Y. Tsui, A. Macphee, D. Hey, P. Patel, A. Mackinnon, M. Key, M. Wei, T. Ma, F. N. Beg, R. Stephens, K. Akli, T. Link, L. Van-Woerkom, and R. R. Freeman. Characterization of the preformed plasma for high-intensity laser-plasma interaction. Opt. Lett., 34(19):2997-2999, Oct 2009. 
[16] Y. Ping, R. Shepherd, B. F. Lasinski, M. Tabak, H. Chen, H. K. Chung, K. B. Fournier, S. B. Hansen, A. Kemp, D. A. Liedahl, K. Widmann, S. C. Wilks, W. Rozmus, and M. Sherlock. Absorption of short laser pulses on solid targets in the ultrarelativistic regime. Phys. Rev. Lett., 100:085004, Feb 2008.

[17] F. N. Beg, A. R. Bell, A. E. Dangor, C. N. Danson, A. P. Fews, M. E. Glinsky, B. A. Hammel, P. Lee, P. A. Norreys, and M. Tatarakis. A study of picosecond laser-solid interactions up to $1019 \mathrm{w}$ cm-2. Phys. Plasmas, 4(2), 1997.

[18] M. G. Haines, M. S. Wei, F. N. Beg, and R. B. Stephens. Hot-electron temperature and laser-light absorption in fast ignition. Phys. Rev. Lett., 102:045008, Jan 2009.

[19] S. C. Wilks, W. L. Kruer, M. Tabak, and A. B. Langdon. Absorption of ultra-intense laser pulses. Phys. Rev. Lett., 69:1383-1386, Aug 1992.

[20] T. Kluge, T. Cowan, A. Debus, U. Schramm, K. Zeil, and M. Bussmann. Electron temperature scaling in laser interaction with solids. Phys. Rev. Lett., 107:205003, Nov 2011.

[21] S. P. Hatchett, C. G. Brown, T. E. Cowan, E. A. Henry, J. S. Johnson, M. H. Key, J. A. Koch, A. B. Langdon, B. F. Lasinski, R. W. Lee, A. J. Mackinnon, D. M. Pennington, M. D. Perry, T. W. Phillips, M. Roth, T. C. Sangster, M. S. Singh, R. A. Snavely, M. A. Stoyer, S. C. Wilks, and K. Yasuike. Electron, photon, and ion beams from the relativistic interaction of petawatt laser pulses with solid targets. Physics of Plasmas (1994-present), 7(5), 2000.

[22] R. A. Snavely, M. H. Key, S. P. Hatchett, T. E. Cowan, M. Roth, T. W. Phillips, M. A. Stoyer, E. A. Henry, T. C. Sangster, M. S. Singh, S. C. Wilks, A. MacKinnon, A. Offenberger, D. M. Pennington, K. Yasuike, A. B. Langdon, B. F. Lasinski, J. Johnson, M. D. Perry, and E. M. Campbell. Intense high-energy proton beams from petawatt-laser irradiation of solids. Phys. Rev. Lett., 85:2945-2948, Oct 2000.

[23] P. Mora. Plasma expansion into a vacuum. Phys. Rev. Lett., 90:185002, May 2003.

[24] A. Macchi, M. Borghesi, and M. Passoni. Ion acceleration by superintense laser-plasma interaction. Rev. Mod. Phys., 85:751-793, May 2013.

[25] H. Daido, M. Nishiuchi, and A. S. Pirozhkov. Review of laser-driven ion sources and their applications. Reports on Progress in Physics, 75(5):056401, 2012.

[26] C. D. Chen. Spectrum and Conversion Efficiency Measurements of Suprathermal Electrons from Relativistic Laser Plasma Interactions. PhD thesis, Massachusetts Institute of Technology, 2009. 
[27] J. Badziak, S. Głowacz, S. Jabłoński, P. Parys, J. Wołowski, and H. Hora. Production of ultrahigh-current-density ion beams by short-pulse skin-layer laser-plasma interaction. Applied Physics Letters, 85(15), 2004.

[28] A. Henig, D. Kiefer, M. Geissler, S. G. Rykovanov, R. Ramis, R. Hörlein, J. Osterhoff, Zs. Major, L. Veisz, S. Karsch, F. Krausz, D. Habs, and J. Schreiber. Laser-driven shock acceleration of ion beams from spherical mass-limited targets. Phys. Rev. Lett., 102:095002, Mar 2009.

[29] T. Esirkepov, M. Borghesi, S. V. Bulanov, G. Mourou, and T. Tajima. Highly efficient relativistic-ion generation in the laser-piston regime. Phys. Rev. Lett., 92:175003, Apr 2004 .

[30] D. W. Schumacher, G. E. Kemp, A. Link, R. R. Freeman, and L. D. Van Woerkom. The shaped critical surface in high intensity laser plasma interactions. Physics of Plasmas (1994-present), 18(1), 2011.

[31] O. Klimo, J. Psikal, J. Limpouch, and V. T. Tikhonchuk. Monoenergetic ion beams from ultrathin foils irradiated by ultrahigh-contrast circularly polarized laser pulses. Phys. Rev. ST Accel. Beams, 11:031301, Mar 2008.

[32] B. Qiao, S. Kar, M. Geissler, P. Gibbon, M. Zepf, and M. Borghesi. Dominance of radiation pressure in ion acceleration with linearly polarized pulses at intensities of $10^{21} \mathrm{~W} \mathrm{~cm}^{-2}$. Phys. Rev. Lett., 108:115002, Mar 2012.

[33] A. Macchi, S. Veghini, T. V Liseykina, and F. Pegoraro. Radiation pressure acceleration of ultrathin foils. New Journal of Physics, 12(4):045013, 2010.

[34] S. Kourtev, N. Minkovski, L. Canova, A. Jullien, O. Albert, and S. M. Saltiel. Improved nonlinear cross-polarized wave generation in cubic crystals by optimization of the crystal orientation. J. Opt. Soc. Am. B, 26(7):1269-1275, Jul 2009.

[35] E. W. Gaul, M. Martinez, J. Blakeney, A. Jochmann, M. Ringuette, D. Hammond, T. Borger, R. Escamilla, S. Douglas, W. Henderson, G. Dyer, A. Erlandson, R. Cross, J. Caird, C. Ebbers, and T. Ditmire. Demonstration of a 1.1 petawatt laser based on a hybrid optical parametric chirped pulse amplification/mixed nd:glass amplifier. Appl. Opt., 49(9):1676-1681, Mar 2010.

[36] E. W. Gaul, N. Kandadai, G. Dyer, T. Borger, M. Martinez, M. Spinks, M. Donovan, and T. Ditmire. Pulse contrast measurements of the texas petawatt laser. In Research in Optical Sciences, page JW2A.23. Optical Society of America, 2014. 
[37] Quere F. Geindre J.-P. Levy A. Ceccotti T. Monot P. Bougeard M. Reau F. d'Oliveira P. Audebert P. Marjoribanks R. Martin Ph. Thaury, C. Plasma mirrors for ultrahighintensity optics. Nat. Phys., 3(6):424-429, 2007.

[38] L. Yin, B. J. Albright, B. M. Hegelich, and J. C. Fernandez. Gev laser ion acceleration from ultrathin targets: The laser break-out afterburner. Laser and Particle Beams, 24:291-298, 62006.

[39] S. Palaniyappan, B. M. Hegelich, H.-C. Wu, D. Jung, D. C. Gautier, L. Yin, B. J. Albright, R. P. Johnson, T. Shimada, S. Letzring, D. T. Offermann, J. Ren, C. Huang, R. Horlein, B. Dromey, J. C. Fernandez, and R. C. Shah. Dynamics of relativistic transparency and optical shuttering in expanding overdense plasmas. Nat. Phys., 8(10):763-769, 2012.

[40] W. Koechner. Solid-State Laser Engineering. Springer, 2006.

[41] Science and technology with ultra-intense lasers. In W. Sandner J. L. Collier G. A. Mourou, G. Korn, editor, ELI Whitebook, ELI. Thoss Media GmbH, 2011.

[42] Z. Chang M. Chini, K. Zhao. The generation, characterization and applications of broadband isolated attosecond pulses. Nat. Photon, 8, 2014.

[43] X.-Y. Li, G.-L. Wang, and X.-X. Zhou. Effects of pressure and gas-jet thickness on the generation of attosecond pulse. Chinese Physics B, 23(1):013102, 2014.

[44] S. Karsch, S. Düsterer, H. Schwoerer, F. Ewald, D. Habs, M. Hegelich, G. Pretzler, A. Pukhov, K. Witte, and R. Sauerbrey. High-intensity laser induced ion acceleration from heavy-water droplets. Phys. Rev. Lett., 91:015001, Jul 2003.

[45] S. Ter-Avetisyan, M. Schnürer, D. Hilscher, U. Jahnke, S. Busch, P. V. Nickles, and W. Sandner. Fusion neutron yield from a laser-irradiated heavy-water spray. Physics of Plasmas (1994-present), 12(1), 2005.

[46] S. Ter-Avetisyan, M. Schnürer, P. V. Nickles, M. Kalashnikov, E. Risse, T. Sokollik, W. Sandner, A. Andreev, and V. Tikhonchuk. Quasimonoenergetic deuteron bursts produced by ultraintense laser pulses. Phys. Rev. Lett., 96:145006, Apr 2006.

[47] M. Schnürer, S. Ter-Avetisyan, P. V. Nickles, and A. A. Andreev. Influence of target system on the charge state, number, and spectral shape of ion beams accelerated by femtosecond high-intensity laser pulses. Physics of Plasmas (1994-present), 14(3), 2007.

[48] J. T. Morrison, M. Storm, E. Chowdhury, K. U. Akli, S. Feldman, C. Willis, R. L. Daskalova, T. Growden, P. Berger, T. Ditmire, L. Van Woerkom, and R. R. Freeman. 
Selective deuteron production using target normal sheath acceleration. Physics of Plasmas (1994-present), 19(3), 2012.

[49] T. Sokollik, M. Schnürer, S. Steinke, P. V. Nickles, W. Sandner, M. Amin, T. Toncian, O. Willi, and A. A. Andreev. Directional laser-driven ion acceleration from microspheres. Phys. Rev. Lett., 103:135003, Sep 2009.

[50] T. Sokollik, T. Paasch-Colberg, K. Gorling, U. Eichmann, M. Schn'urer, S. Steinke, P. V. Nickles, A. Andreev, and W. Sandner. Laser-driven ion acceleration using isolated mass-limited spheres. New Journal of Physics, 12(11):113013, 2010.

[51] A. P. Higginbotham, O. Semonin, S. Bruce, C. Chan, M. Maindi, T. D. Donnelly, M. Maurer, W. Bang, I. Churina, J. Osterholz, I. Kim, A. C. Bernstein, and T. Ditmire. Generation of mie size microdroplet aerosols with applications in laserdriven fusion experiments. Review of Scientific Instruments, 80(6), 2009.

[52] S. Ter-Avetisyan, M. Schnürer, H. Stiel, and P. V. Nickles. A high-density sub-micron liquid spray for laser driven radiation sources. Journal of Physics D: Applied Physics, $36(19): 2421,2003$.

[53] S. Ter-Avetisyan, B. Ramakrishna, R. Prasad, M. Borghesi, P. V. Nickles, S. Steinke, M. Schnürer, K. I. Popov, L. Ramunno, N. V. Zmitrenko, and V. Yu. Bychenkov. Generation of a quasi-monoergetic proton beam from laser-irradiated sub-micron droplets. Physics of Plasmas (1994-present), 19(7), 2012.

[54] T. Nayuki, Y. Oishi, T. Fujii, K. Nemoto, T. Kayoiji, Y. Okano, Y. Hironaka, K. G. Nakamura, K. Kondo, and K. Ueda. Thin tape target driver for laser ion accelerator. Review of Scientific Instruments, 74(7), 2003.

[55] J. Osterhoff E. Evans A. J. Gonsalves K. Nakamura J. van Tilborg C. Lin C. Toth T. Sokollik, S. Shiraishi and W. P. Leemans. A high repetition rate plasma mirror for staged electron acceleration. Number MOP084, pages 256-258, 2011.

[56] Y. Oishi, T. Nayuki, T. Fujii, Y. Takizawa, X. Wang, T. Yamazaki, K. Nemoto, T. Kayoiji, T. Sekiya, K. Horioka, Y. Okano, Y. Hironaka, K. G. Nakamura, K. Kondo, and A. A. Andreev. Dependence on laser intensity and pulse duration in proton acceleration by irradiation of ultrashort laser pulses on a cu foil target. Physics of Plasmas (1994-present), 12(7), 2005.

[57] S. Feister, J. A. Nees, J. T. Morrison, K. D. Frische, C. Orban, E. A. Chowdhury, and W. M. Roquemore. A novel femtosecond-gated, high-resolution, frequency-shifted shearing interferometry technique for probing pre-plasma expansion in ultra-intense laser experimentsa). Review of Scientific Instruments, 85(11), 2014. 
[58] M. Faubel, B. Steiner, and J. P. Toennies. Photoelectron spectroscopy of liquid water, some alcohols, and pure nonane in free micro jets. The Journal of Chemical Physics, 106(22), 1997.

[59] J. T. Morrison, E. A. Chowdhury, K. D. Frische, S. Feister, V. M. Ovchinnikov, J. A. Nees, C. Orban, R. R. Freeman, and W. M. Roquemore. Backward-propagating mev electrons from $10^{18} \mathrm{w} / \mathrm{cm}^{2}$ laser interactions with water. arXiv, 2015.

[60] D. Panasenko, A. J. Shu, A. Gonsalves, K. Nakamura, N. H. Matlis, C. Toth, and W. P. Leemans. Demonstration of a plasma mirror based on a laminar flow water film. Journal of Applied Physics, 108(4), 2010.

[61] M. Trebbin, K. Kruger, D. DePonte, S. V. Roth, H. N. Chapman, and S. Forster. Microfluidic liquid jet system with compatibility for atmospheric and high-vacuum conditions. Lab Chip, 14:1733-1745, 2014.

[62] A. V. Brantov, V. T. Tikhonchuk, O. Klimo, D. V. Romanov, S. Ter-Avetisyan, M. Schnürer, T. Sokollik, and P. V. Nickles. Quasi-mono-energetic ion acceleration from a homogeneous composite target by an intense laser pulse. Physics of Plasmas (1994-present), 13(12), 2006.

[63] U. Zastrau, P. Sperling, M. Harmand, A. Becker, T. Bornath, R. Bredow, S. Dziarzhytski, T. Fennel, B. Fletcher, L. E. Förster, S. Göde, G. Gregori, V. Hilbert, D. Hochhaus, B. Holst, T. Laarmann, J. Lee, H. T. Ma, P. Mithen, J. R. Mitzner, D. Murphy, C. M. Nakatsutsumi, P. Neumayer, A. Przystawik, S. Roling, M. Schulz, B. Siemer, S. Skruszewicz, J. Tiggesbäumker, S. Toleikis, T. Tschentscher, T. White, M. Wöstmann, H. Zacharias, T. Döppner, H. Glenzer, S. and R. Redmer. Resolving ultrafast heating of dense cryogenic hydrogen. Phys. Rev. Lett., 112:105002, Mar 2014.

[64] D. Strickland and G. Mourou. Compression of amplified chirped optical pulses. Optics Communications, 55:447-449, oct 1985.

[65] M. D. Perry, D. Pennington, B. C. Stuart, G. Tietbohl, J. A. Britten, C. Brown, S. Herman, B. Golick, M. Kartz, J. Miller, H. T. Powell, M. Vergino, and V. Yanovsky. Petawatt laser pulses. Opt. Lett., 24(3):160-162, Feb 1999.

[66] V. Yanovsky, V. Chvykov, G. Kalinchenko, P. Rousseau, T. Planchon, T. Matsuoka, A. Maksimchuk, J. Nees, G. Cheriaux, G. Mourou, and K. Krushelnick. Ultra-high intensity- 300-tw laser at $0.1 \mathrm{hz}$ repetition rate. Opt. Express, 16(3):2109-2114, Feb 2008.

[67] S. H. Batha, R. Aragonez, F. L. Archuleta, T. N. Archuleta, J. F. Benage, J. A. Cobble, J. S. Cowan, V. E. Fatherley, K. A. Flippo, D. C. Gautier, R. P. 
Gonzales, S. R. Greenfield, B. M. Hegelich, T. R. Hurry, R. P. Johnson, J. L. Kline, S. A. Letzring, E. N. Loomis, F. E. Lopez, S. N. Luo, D. S. Montgomery, J. A. Oertel, D. L. Paisley, S. M. Reid, P. G. Sanchez, A. Seifter, T. Shimada, and J. B. Workman. Trident high-energy-density facility experimental capabilities and diagnosticsa). Review of Scientific Instruments, 79(10), 2008.

[68] Y. B. Zel'dovich. Interaction of free electrons with electromagnetic radiation. Soviet Physics Uspekhi, 18(2):79, 1975.

[69] A. E. Siegman. Lasers. University Science Books, 1986.

[70] P. S. Banks, M.D. Perry, V. Yanovsky, S.N. Fochs, B.C. Stuart, and J. Zweiback. Novel all-reflective stretcher for chirped-pulse amplification of ultrashort pulses. Quantum Electronics, IEEE Journal of, 36(3):268-274, 2000.

[71] Paul T. Konkola, Carl G. Chen, Ralf K. Heilmann, Chulmin Joo, Juan C. Montoya, Chih-Hao Chang, and Mark L. Schattenburg. Nanometer-level repeatable metrology using the nanoruler. Journal of Vacuum Science ES Technology B, 21(6), 2003.

[72] P. L. Poole, S. Trendafilov, G. Shvets, D. Smith, and E. Chowdhury. Femtosecond laser damage threshold of pulse compression gratings for petawatt scale laser systems. Opt. Express, 21(22):26341-26351, Nov 2013.

[73] B. C. Walker, C. Tóth, D. Fittinghoff, and T. Guo. Theoretical and experimental spectral phase error analysis for pulsed laser fields. J. Opt. Soc. Am. B, 16(8):12921299, Aug 1999.

[74] S. Luan, M. H. R. Hutchinson, R. A. Smith, and F. Zhou. High dynamic range thirdorder correlation measurement of picosecond laser pulse shapes. Measurement Science and Technology, 4(12):1426, 1993.

[75] C. Willis, P. L. Poole, K. U. Akli, D. W. Schumacher, and R. R. Freeman. A confocal microscope position sensor for micron-scale target alignment in ultra-intense lasermatter experiments. RSI submitted, 2015.

[76] K. U. Akli, P. K. Patel, R. Van Maren, R. B. Stephens, M. H. Key, D. P. Higginson, B. Westover, C. D. Chen, A. J. Mackinnon, T. Bartal, F. N. Beg, S. Chawla, R. Fedosejevs, R. R. Freeman, D. S. Hey, G. E. Kemp, S. LePape, A. Link, T. Ma, A. G. MacPhee, H. S. McLean, Y. Ping, Y. Y. Tsui, L. D. Van Woerkom, M. S. Wei, T. Yabuuchi, and S. Yuspeh. A dual channel x-ray spectrometer for fast ignition research. Journal of Instrumentation, 5(07):P07008, 2010. 
[77] J. T. Morrison, C. Willis, R. R. Freeman, and L. Van Woerkom. Design of and data reduction from compact thomson parabola spectrometers. Review of Scientific Instruments, 82(3), 2011.

[78] J. P. Chambaret, F. Mathieu, and K. Osvay. Apollon building stage has begun. ELI Courier, 2, 2010.

[79] B. N. Chichkov, C. Momma, S. Nolte, F. Von Alvensleben, , and A. Tünnermann. Femtosecond, picosecond and nanosecond laser ablation of solids. APA, (63):109-115, 1996.

[80] E. G. Gamaly, A. V. Rode, B. Luther-Davies, and V. T. Tikhonchuk. Ablation of solids by femtosecond lasers: Ablation mechanism and ablation thresholds for metals and dielectrics. Physics of Plasmas (1994-present), 9(3), 2002.

[81] B. C. Stuart, M. D. Feit, S. Herman, A. M. Rubenchik, B. W. Shore, and M. D. Perry. Optical ablation by high-power short-pulse lasers. J. Opt. Soc. Am. B, 13(2):459-468, Feb 1996.

[82] R. D. Boyd, J. A. Britten, D. E. Decker, B. W. Shore, B. C. Stuart, M. D. Perry, and Lifeng Li. High-efficiency metallic diffraction gratings for laser applications. Appl. Opt., 34(10):1697-1706, Apr 1995.

[83] J. A. Britten, M. D. Perry, B. W. Shore, and R. D. Boyd. Universal grating design for pulse stretching and compression in the 800-1100-nm range. Opt. Lett., 21(7):540-542, Apr 1996.

[84] S.-S. Wellershoff, J. Hohlfeld, J. Güdde, and E. Matthias. The role of electron-phonon coupling in femtosecond laser damage of metals. Applied Physics A, 69(1):S99-S107, 1999.

[85] P.P Pronko, S.K Dutta, J Squier, J.V Rudd, D Du, and G Mourou. Machining of sub-micron holes using a femtosecond laser at $800 \mathrm{~nm}$. Optics Communications, 114(1-2):106 - 110, 1995.

[86] X. Liu, D. Du, and G. Mourou. Laser ablation and micromachining with ultrashort laser pulses. Quantum Electronics, IEEE Journal of, 33(10):1706-1716, Oct 1997.

[87] N. Bonod and J. Neauport. Optical performance and laser induced damage threshold improvement of diffraction gratings used as compressors in ultra high intensity lasers. Optics Communications, 260(2):649 - 655, 2006. 
[88] F. Canova, R. Clady, J.-P. Chambaret, M. Flury, S. Tonchev, R. Fechner, and O. Parriaux. High-efficiency, broad band, high-damage threshold high-index gratings for femtosecond pulse compression. Opt. Express, 15(23):15324-15334, Nov 2007.

[89] J. Neauport, E. Lavastre, G. Razé, G. Dupuy, N. Bonod, M. Balas, G. de Villele, J. Flamand, S. Kaladgew, and F. Desserouer. Effect of electric field on laser induced damage threshold of multilayer dielectric gratings. Opt. Express, 15(19):12508-12522, Sep 2007.

[90] J. Neauport, N. Bonod, S. Hocquet, S. Palmier, and G. Dupuy. Mixed metal dielectric gratings for pulse compression. Opt. Express, 18(23):23776-23783, 2010.

[91] A. Cotel, N. Forget, C. Brach, F. Bonnemason, E. Baynard, C. Le Bris, and C. Le Blanc. Characterisation of multilayer dielectric gratings for petawatt-class lasers. In Conference on Lasers and Electro-Optics/Quantum Electronics and Laser Science and Photonic Applications Systems Technologies, page JFB6. Optical Society of America, 2005.

[92] F. Kong, Y. Jin, S. Liu, S. Chen, H. Guan, K. He, Y. Du, and H. He. Femtosecond laser damage of broadband pulse compression gratings. Chin. Opt. Lett., 11(10):102302, Oct 2013 .

[93] E. Gubbini, G. Kommol, M. Schnürer, H. Schönagel, U. Eichmann, M.P. Kalashnikov, P.V. Nickles, F. Eggenstein, G. Reichardt, and W. Sandner. "on-line" cleaning of optical components in a multi-tw-ti:sa laser system. Vacuum, 76(1):45 - 49, 2004.

[94] A. J. Langley, W. J. Lester, and J. M. Smith. Rf plasma cleaning of compression gratings for intense femtosecond pulses. Technical report, 2003.

[95] G. Xulei, T. Hao, Z. Yi, M. Jinglong, Z. Wei, M. Jingyi, C. Liming, W. Zhaohua, L. Yutong, J. Gang, H. Duanwei, , and W. Zhiyi. Plasma cleaning of compressed grating in chirped-pulse femtosecond laser amplifier. Chinese J. Lasers, 04, 2012.

[96] T. Jitsuno, H. Murakami, S. Motokoshi, E. Saato, K. Mikami, K. Kato, T. Kawasaki, Y. Nakata, N. Sarukura, T. Shinizu, H. Shiraga, N. Miyanaga, and H. Azechi. Oilcontamination problem in large-scale pulse-compressor. volume 7842, page 784221 , 2010.

[97] E. G. Loewen and E. Popov. Diffraction Gratings and Applications. Marcel Dekker, Inc.

[98] J. Bonse, S. Baudach, W. Kautek, E. Welsch, and J. Krüger. Femtosecond laser damage of a high reflecting mirror. Thin Solid Films, 408(1-2):297 - 301, 2002. 
[99] G. Batavic̃iutè, P. Grigas, L. Smalakys, and A. Melninkaitis. Bayesian approach of laser-induced damage threshold analysis and determination of error bars, 2012.

[100] X.C. Wang, G.C. Lim, H.Y. Zheng, F.L. Ng, W. Liu, and S.J. Chua. Femtosecond pulse laser ablation of sapphire in ambient air. Applied Surface Science, 228(1-4):221 $-226,2004$.

[101] D. Ashkenasi, M. Lorenz, R. Stoian, and A. Rosenfeld. Surface damage threshold and structuring of dielectrics using femtosecond laser pulses: the role of incubation. Applied Surface Science, 150(1-4):101 - 106, 1999.

[102] H. Chen, X. Chen, Y. Zhang, and Y. Xia. Ablation induced by single-and multiplefemtosecond laser pulses in lithium niobate. Laser Physics, 17(12):1378-1381, 2007.

[103] J. Kruger, D. Dufft, R. Koter, and A. Hertwig. Femtosecond laser-induced damage of gold films. Applied Surface Science, 253(19):7815 - 7819, 2007. Photon-Assisted Synthesis and Processing of Functional Materials E-MRS-H Symposium.

[104] B. Wang and L. Gallais. A theoretical investigation of the laser damage threshold of metal multi-dielectric mirrors for high power ultrashort applications. Opt. Express, 21(12):14698-14711, Jun 2013.

[105] M. Mero, B. Clapp, J. C. Jasapara, W. Rudolph, D. Ristau, K. Starke, J. Kruger, S. Martin, and W. Kautek. On the damage behavior of dielectric films when illuminated with multiple femtosecond laser pulses. Optical Engineering, 44(5):051107-051107-7, 2005.

[106] L. A. Emmert, M. Mero, and W. Rudolph. Modeling the effect of native and laserinduced states on the dielectric breakdown of wide band gap optical materials by multiple subpicosecond laser pulses. Journal of Applied Physics, 108(4), 2010.

[107] E. D. Palik. Handbook of Optical Constants of Solids. Academic Press, 1985.

[108] V. D. Vinokurova, R. R. Gerke, T. G. Dubrovina, M. D. Mikhailov, E. G. Sall', Aleksandr V. Charukhchev, and V. E. Yashin. Metallised holographic diffraction gratings with the enhanced radiation resistance for laser pulse compression systems. Quantum Electronics, 35(6):569, 2005.

[109] P. J. Collings and M. Hird. Introduction to liquid crystals: Chemistry and Physics. Taylor and Francis Group, 1997.

[110] J. L. Serrano, editor. Metallomesogens: Synthesis, Properties, and Applications. VCH publishers, Inc, 1996. 
[111] N. V. Madhusudana and R. Pratibha. Elasticity and orientational order in some cyanobiphenyls: Part iv. reanalysis of the data. Molecular Crystals and Liquid Crystals, 89(1-4):249-257, 1982.

[112] M. Mitra. Orientational ordering from molecular polarizabilities and an odd-even effect in ncb homologous series. Phase Transitions, 37(2-3):131-140, 1992.

[113] E. D. Palik. Smectic and Columnar Liquid Crystals: Concepts and Physical Properties Illustrated by Experiments. Taylor and Francis Group, 2006.

[114] S. Kumar. Liquid Crystals: Experimental Study of Physical Properties and Phase Transitions. Cambridge University Press, 2001.

[115] G. Chilaya. Cholesteric liquid crystals: Optics, electro-optics, and photo-optics. In Heinz-Siegfried Kitzerow and Christian Bahr, editors, Chirality in Liquid Crystals, Partially Ordered Systems, pages 159-185. Springer New York, 2001.

[116] A. A. Sonin. Freely Suspended Liquid Crystalline Films. Wiley, 1999.

[117] C. Rosenblatt, R. Pindak, N. A. Clark, and R. B. Meyer. Freely suspended ferroelectric liquid-crystal films: Absolute measurements of polarization, elastic constants, and viscosities. Phys. Rev. Lett., 42:1220-1223, Apr 1979.

[118] S. W. Morris, J. R. de Bruyn, and A. D. May. Electroconvection and pattern formation in a suspended smectic film. Phys. Rev. Lett., 65:2378-2381, Nov 1990.

[119] D. R. Link, J. E. Maclennan, and N. A. Clark. Simultaneous observation of electric field coupling to longitudinal and transverse ferroelectricity in a chiral liquid crystal. Phys. Rev. Lett., 77:2237-2240, Sep 1996.

[120] Z. A. Daya, S. W. Morris, and J. R. de Bruyn. Electroconvection in a suspended fluid film: A linear stability analysis. Phys. Rev. E, 55:2682-2692, Mar 1997.

[121] D. Pettey, T. C. Lubensky, and D. R. Link. Topological inclusions in 2d smectic c films. Liquid Crystals, 25(5):579-587, 1998.

[122] C. Bohley and R. Stannarius. Inclusions in free standing smectic liquid crystal films. Soft Matter, 4:683-702, 2008.

[123] Z. A. Daya. Electroconvection in Sheared Annular Fluid Films. PhD thesis, University of Toronto, 2000.

[124] L. J. Atkins and R. C. Elliott. Investigating thin film interference with a digital camera. American Journal of Physics, 78(12), 2010. 
[125] R. G. Horn. Refractive indices and order parameters of two liquid crystals. J. Phys. France, 39(1), 1977.

[126] A. Pattanaporkratana, C. S. Park, J. E. Maclennan, and N. A. Clark. Manipulation of disk-shaped islands on freely suspended smectic films and bubbles using optical tweezers. Ferroelectrics, 310(1):131-135, 2004.

[127] P. Pieranski, L. Beliard, J.-Ph. Tournellec, X. Leoncini, C. Furtlehner, H. Dumoulin, E. Riou, B. Jouvin, J.-P. Fénerol, Ph. Palaric, J. Heuving, B. Cartier, and I. Kraus. Physics of smectic membranes. Physica A: Statistical Mechanics and its Applications, 194(1-4):364 - 389, 1993.

[128] I. J. Kim, K. H. Pae, C. M. Kim, H. T. Kim, J. H. Sung, S. K. Lee, T. J. Yu, I. W. Choi, C.-L. Lee, K. H. Nam, P. V. Nickles, T. M. Jeong, and J. Lee. Transition of proton energy scaling using an ultrathin target irradiated by linearly polarized femtosecond laser pulses. Phys. Rev. Lett., 111:165003, Oct 2013.

[129] A. Macchi, S. Veghini, and F. Pegoraro. "light sail" acceleration reexamined. Phys. Rev. Lett., 103:085003, Aug 2009.

[130] S. Gaillard, J. Fuchs, N. Renard-Le Galloudec, and T. E. Cowan. Study of saturation of cr39 nuclear track detectors at high ion fluence and of associated artifact patterns. Review of Scientific Instruments, 78(1), 2007.

[131] C. Palmer, C. Bellei, A. E. Dangor, S. Kneip, S. P. D. Mangles, S. R. Nagel, Z. Nagmudin, L. Willingale, R. J. Clarke, R. Heathcote, A. Henig, J. Schreiber, M. C. Kaluza, and A. Savart. Proton energy spectra from ultra-intense laser interactions with film targets of varying thicknesses. Technical report, 2007.

[132] F. Fiuza, A. Stockem, E. Boella, R. A. Fonseca, L. O. Silva, D. Haberberger, S. Tochitsky, W. B. Mori, and C. Joshi. Ion acceleration from laser-driven electrostatic shocksa). Physics of Plasmas (1994-present), 20(5), 2013.

[133] A. P. L Robinson, A. R. Bell, and R. J. Kingham. Effect of target composition on proton energy spectra in ultraintense laser-solid interactions. Phys. Rev. Lett., 96:035005, Jan 2006.

[134] W. P. Leemans, A. J. Gonsalves, H.-S. Mao, K. Nakamura, C. Benedetti, C. B. Schroeder, Cs. Tóth, J. Daniels, D. E. Mittelberger, S. S. Bulanov, J.-L. Vay, C. G. R. Geddes, and E. Esarey. Multi-gev electron beams from capillary-discharge-guided subpetawatt laser pulses in the self-trapping regime. Phys. Rev. Lett., 113:245002, Dec 2014. 
[135] P. L. Poole, C. D. Andereck, D. W. Schumacher, R. L. Daskalova, S. Feister, K. M. George, C. Willis, K. U. Akli, and E. A. Chowdhury. Liquid crystal films as ondemand, variable thickness (50-5000) targets for intense lasers. Physics of Plasmas (1994-present), 21(6), 2014. 